\title{
The role of galectin-3 in inflammation.
}

\author{
Sarah Farnworth
}

A thesis submitted for the degree of Doctor of Philosophy

University of Edinburgh, 2007 
Dedicated to my parents Michael and Ann. 


\section{Declaration}

I hereby declare that this thesis has been composed solely by myself and has not been accepted in any previous candidature for a higher degree. All work presented in this thesis was, unless acknowledged, initiated and executed by myself. Chapter 4 of this thesis presents work carried out in collaboration with Dr Neil Henderson with substantial contribution from myself. All sources of information in the text have been acknowledged by reference.

Sarah Farnworth

February 2008 


\begin{abstract}
Galectin-3, a unique member of the growing family of $\beta$-galactoside binding lectins, contains a single carbohydrate recognition domain and a glycine rich Nterminal domain through which it can form oligomers and functions to cross-link both carbohydrate and non-carbohydrate ligands. Galectin-3 is widely expressed in adult tissues, particularly on and secreted by activated macrophages and monocytes. Galectin-3 has been implicated in many facets of the inflammatory response including neutrophil and macrophage activation and function.

In this thesis I have examined the role of galectin-3 during fibrosis, alternative activation of macrophages and pneumonia. Galectin-3 expression is upregulated in established human fibrotic liver disease and in a mouse model of liver fibrosis induced by carbon tetrachloride. Galectin-3 expression is temporally and spatially related to the induction and resolution of experimental hepatic fibrosis in this model. In addition, disruption of the galectin-3 gene markedly attenuates liver and kidney fibrosis, induced by unilateral ureteric obstruction, with reduced collagen deposition and myofibroblast activation. Results suggest that galectin-3 may promote fibrosis by stimulating myofibroblast activation by a transforming growth factor- $\beta$ (TGF- $\beta$ )-independent mechanism.

Recent reports suggest that alternative macrophage activation is one of the key steps toward the progression of fibrosis. Disruption of the galectin-3 gene specifically restrains interleukin-4 (IL-4)/IL-13-induced alternative macrophage activation in vitro. My results suggest that the key mechanism required for activation of an alternative macrophage phenotype is an IL-4-stimulated galectin-3 feed back loop which directly activates CD98 causing sustained phosphatidylinositol 3-kinase (PI3-K) activation.

The gram-positive Streptococcus pneumoniae $(S . \mathrm{pn})$ is the leading cause of community acquired pneumonia worldwide, resulting in high mortality. Galectin- $3^{-/-}$ mice demonstrate a clearance defect of $S$. $p n$ with increased septicaemia and a greater extent of lung damage compared to wild type mice. This phenotype is markedly reduced in pneumonia induced by the gram-negative Escherichia coli (E.
\end{abstract}


coli). I have shown that presence of galectin-3 reduces the severity of pneumonia induced by $S$. pn and this is achieved through a number of processes: 1) Galectin-3 has bactericidal properties towards $S$. pn in vitro. 2) Galectin- $3^{-/-}$macrophages show reduced production of nitrite following incubation with both $S$. pn and E. coli and hence a reduction in bacterial killing. 3) Galectin-3 activates neutrophils to produce reactive oxygen species which enhances the bactericidal activity of neutrophils. 4) Activation of neutrophils by galectin-3 augments phagocytosis of bacteria. 5) Finally, initial data suggests that galectin- $3^{-/-}$neutrophils apoptose more readily than wild type neutrophils in vitro and galectin $-3^{-/-}$macrophages phagocytose apoptotic neutrophils less efficiently compared to wild type. In vivo this would result in an accumulation of dying cells in the lung. The damage these apoptotic cells would have on the lung tissue may enable the bacteria to enter the blood stream resulting in sepsis.

In summary, in response to chronic tissue injury, persistant upregulation of galectin-3 causes myofibroblast and alternative macrophage activation, thus enhancing collagen deposition and scarring. However during an acute $S$. pn infection, galectin-3 plays a benefitial role to aid the clearance of bacteria through a variety of processes. Therefore, galectin-3 plays a critical role in a variety of inflammatory disorders. 


\section{Acknowledgements}

I would like to thank the Medical Research Council for providing the 3 years of funding to carry out this research.

I am grateful to Professor Tariq Sethi for inviting me into this excellent, hard working research group and providing me with advice and support throughout my $\mathrm{PhD}$ while allowing me to explore my own lines of research. I am also appreciative of my second supervisor Dr Simon Hart for giving me scientific advice when needed.

I would like to acknowledge the people who have helped me set up experiments and given me advice and assistance throughout my $\mathrm{PhD}$.

I would like to thank Professor Chris Gregory's group for giving me initial advice for setting up the baculovirus expression system detailed in chapter 3 .

The $\mathrm{CCl}_{4}$ rat model of liver fibrosis was established by Professor John Iredale (University of Edinburgh). Our lab modified this model for use in mouse to carry out the in vivo work detailed in chapter 4 of this thesis. This work was carried out in collaboration with Dr Neil Henderson who performed the majority of the in vivo injections. In vitro protocols (including immunohistochemistry, real-time RT-PCR, siRNA and Western blotting) were performed by myself and Dr Neil Henderson in equal measure. The UUO model of kidney fibrosis had been used previously by Dr Jeremy Hughes' group (University of Edinburgh) and was kindly performed by Spike Clay. Kidneys were harvested by myself and Dr Alison Mackinnon. I would also like to thank the University of Edinburgh Department of Pathology for providing the human liver samples, Professor John Iredale for providing the rat liver sections and the Histology Department at the University of Edinburgh for paraffin embedding the mouse tissue samples and preparing sections for staining. Lastly I must thank Dr Gareth Clegg for helping me to set up the staining quantitation program using Openlab. 
The in vivo models of infection detailed in chapter 6 were chosen due to the presence of expertise in the Centre for Inflammation Research (University of Edinburgh) at intratracheal administration of substances to mice. Dr Neil Henderson and I planned and carried out these in vivo experiments with the help of the colleagues from Dr John Simpson's laboratory - Lesley Farrell (intratracheal administration of bacteria and bronchoalveolar lavages) and Dr Tom Wilkinson (assisted with plating out samples for bacterial detection on agar plates). Kirsten Atkinson helped me to set up the in vitro antibacterial assay and assisted me in counting colonies. I would like to thank Professor Adriano Rossi for assisting me in setting up the neutrophil activation assays and Dr Brian McHugh and Shonna Johnston for confocal and flow cytometry guidance.

I must emphasise my appreciation towards Dr Alison Mackinnon for her patience and supervision during my time in the lab and during the write-up period. She has been instrumental to my success as a scientist and is a good friend. And for her excellent technical assistance I would like to thank Kirsten Atkinson for looking after my cells on occasion and assisting me in some of my in vitro work when needed.

Many many thanks to my mum and dad who have always allowed me to make my own decisions and given me continuous support and encouragement in whatever I take on. Big thanks to my sister and best friend Vicky, a fountain of good advice and great tap teacher to boot.

And finally, a huge thanks and lots of love to my partner and best friend Brian for support, guidance, patience and laughter both in "the before time" and in the past year. 


\section{Abbreviations}

$\alpha$-SMA

$\mathrm{ABC}$

Akt/PKB

APC

APS

BAL

$\beta$-Me

BMDM

bp

BSA

$\mathrm{Ca}^{2+}$

CAP

CBA

$\mathrm{CCl}_{4}$

cDNA

CFU

CRD

DAB

DAPI

$\mathrm{dH}_{2} \mathrm{O}$

DHR

DMEM

DMSO

DNA

dNTP

DT

DTR

DTT

E. coli

ECL

ECM

EDTA

ELISA

EMT

EPDA

ERK

FACS

FBS

FCS

FITC

FIZZ-1

fMLP

FSG
Alpha-smooth muscle actin

Avidin:biotinylated enzyme complex

Protein kinase B

Allophycocyanin or antigen presenting cell

Ammonium persulphate

Bronchoalveolar lavage

$\beta$-mercaptoethanol

Bone marrow derived macrophage

Base pair

Bovine serum albumin

Calcium

Community acquired pneumonia

Cytometric bead array

Carbon tetrachloride

Complementary DNA

Colony forming units

Carbohydrate recognition domain

Diaminobenzidine

4', 6'-diamidino-2-phenylindole

Distilled $\mathrm{H}_{2} \mathrm{O}$

Dihydrorhodamine

Dulbecco's modified eagles medium

Dimethyl sulfoxide

Deoxyribonucleic acid

Deoxynucleotide triphosphate

Diptheria toxin

Diptheria toxin receptor

Dithiothreitol

Escherichia coli

Enhanced chemiluminescence

Extracellular matrix

Ethylenediaminetetraacetic acid

Enzyme-linked immuno sorbent assay

Epithelial to mesenchymal transition

End point dilution assay

Extracellular signal-regulated kinase

Fluorescence activated cell sorter

Foetal bovine serum

Foetal calf serum

Fluorescein isothiocyanate

Found in inflammatory zone-1

N-Formyl-Methionine-Leucine-Phenylalanine

Fish skin gelatine 


\begin{tabular}{|c|c|}
\hline Galectin--3/- & Galectin-3 knockout \\
\hline GMCSF & Granulocyte-macrophage colony-stimulating factor \\
\hline $\mathrm{H}_{2} \mathrm{O}_{2}$ & Hydrogen peroxide \\
\hline $\mathrm{H} \& \mathrm{E}$ & Haematoxylin and Eosin \\
\hline HBSS & Hanks' balanced salt solution \\
\hline $\operatorname{HBSS}(+)$ & Hanks' balanced salt solution plus $\mathrm{Ca}^{2+}$ and $\mathrm{Mg}^{2+}$ \\
\hline HEPES & 4-(2-hydroxyethyl)-1-piperazine ethanesulfonic acid \\
\hline HIB-S & Heat treated horse serum \\
\hline His & Histidine \\
\hline HRP & Horse radish peroxidase \\
\hline HSC & Hepatic stellate cell \\
\hline $\mathrm{Hu}$ & Human \\
\hline IFN $\gamma$ & Interferon gamma \\
\hline $\operatorname{Ig}$ & Immunoglobulin \\
\hline $\mathrm{IHC}$ & Immunohistochemistry \\
\hline IL-4 & Interleukin-4 \\
\hline IL-13 & Interleukin-13 \\
\hline IMDM & Iscove's modified eagles medium \\
\hline IMF & Immunofluorescence \\
\hline i.p. & Intraperitoneal \\
\hline IPTG & Isopropyl- $\beta$-D-thiogalactopyranoside \\
\hline ISPF & $\alpha$-isonitrosopropiophenone \\
\hline i.t. & Intratracheal \\
\hline Jak & Janus kinase \\
\hline JNK & Jun N-terminal kinase \\
\hline $\mathrm{Kb}$ & Kilobase \\
\hline $\mathrm{KDa}$ & Kilodalton \\
\hline LB & Luria-Bertani \\
\hline LPS & Lipopolysaccharide \\
\hline MAPK & Mitogen activated protein kinase \\
\hline MEK & MAPK/ERK kinase \\
\hline $\mathrm{Mg}^{2+}$ & Magnesium \\
\hline MMP & Matrix metalloproteinase \\
\hline mRNA & Messenger RNA \\
\hline Ms & Mouse \\
\hline MTT & 3-(4,5-dimethylthiazol-2yl)-2,5-diphenyl tetrazolium bromide \\
\hline${ }_{\mathrm{NF}} \mathrm{H}_{2} \mathrm{O}$ & Nuclease-free $\mathrm{H}_{2} \mathrm{O}$ \\
\hline NO & Nitric oxide \\
\hline NOS2 & Inducible nitric oxide synthase \\
\hline $\mathrm{O}^{2-}$ & Superoxide \\
\hline OD & Optical density \\
\hline PAF & Platelet-activating factor \\
\hline PBS & Phosphate buffered saline \\
\hline PCR & Polymerase chain reaction \\
\hline PDGF & Platelet-derived growth factor \\
\hline $\mathrm{PE}$ & Phycoerythrin \\
\hline PFA & Paraformaldehyde \\
\hline PI3-kinase & Phosphatidylinositol 3-kinase \\
\hline
\end{tabular}




$\begin{array}{ll}\text { PKC } & \text { Protein kinase C } \\ \text { PMA } & \text { Phorbol 12-myristate 13-acetate } \\ \text { PMN } & \text { Polymorphonuclear leukocyte } \\ \text { PSR } & \text { Picrosirius red } \\ \text { RGB } & \text { Red, green and blue } \\ \text { RNA } & \text { Ribonucleic acid } \\ \text { ROS } & \text { Reactive oxygen species } \\ \text { rRNA } & \text { Ribosomal RNA } \\ \text { RT PCR } & \text { Reverse Transcription PCR } \\ \text { SDS-PAGE } & \text { Sodium dodecyl-sulphate polyacrylamide gel electrophoresis } \\ \text { SHIP } & \text { Src homology 2-containing inositol-5'-phosphate } \\ \text { siRNA } & \text { Small interfering RNA } \\ \text { S.O.B } & \text { Super optimal broth } \\ \text { S.O.C } & \text { S.O.B plus 20mM glucose (the C stands for catabolite repression, } \\ & \text { reflective of the added glucose) } \\ \text { STAT } & \text { Signal transducers and activators of transcription } \\ \text { S. } p n & \text { Streptococcal pneumoniae } \\ \text { TAE } & \text { Tris-acetate-EDTA } \\ \text { TBS } & \text { Tris buffered saline } \\ \text { TCR } & \text { T-cell receptor } \\ \text { TEMED } & \text { Tetramethylethylenediamine } \\ \text { TGF- } \beta & \text { Transforming growth factor-beta } \\ \text { Th1/2 } & \text { T-helper 1/2 } \\ \text { TNF- } \alpha & \text { Tumour necrosis factor-alpha } \\ \text { UUO } & \text { Unilateral ureteric obstruction } \\ \text { WT } & \text { Wild type } \\ \text { X-gal } & \text { 5-bromo-4-chloro-3-indolyl- } \beta \text {-D-galactopyranoside } \\ & \end{array}$




\section{Table of Contents}

DECLARATION

ABSTRACT III

ACKNOWLEDGMENTS $\quad$ V

ABBREVIATIONS $\quad$ VII

CHAPTER 1 1

INTRODUCTION

1.1 Galectins 1

1.1.1 Background 1

1.1.2 Origin of the term "galectin" 1

1.1.3 The galectin family $\quad \mathbf{2}$

1.2 Galectin-3 4

1.2.1 Distribution and localisation $\quad 4$

1.2.2 Regulation of galectin-3 expression $\quad \mathbf{5}$

1.2.3 Intracellular binding partners, functions and signalling $\quad \mathbf{6}$

$\begin{array}{rr}\text { 1.2.4 Extracellular functions } & \mathbf{8}\end{array}$

$\begin{array}{lll}1.2 .5 & \text { Cell adhesion } & \mathbf{1 0}\end{array}$

1.2.6 Regulation of the immune response $\quad \mathbf{1 1}$

$\begin{array}{lll}\text { 1.2.7 Galectin-3 and cancer } & \mathbf{1 4}\end{array}$

$\begin{array}{lll}1.2 .8 & \text { CD98 } & \mathbf{1 5}\end{array}$

$\begin{array}{lll}\text { 1.2.9 The galectin- } 3^{-/-} \text {mouse } & \mathbf{1 6}\end{array}$

1.3 Chronic inflammation and infection 19

$\begin{array}{llr}1.3 .1 & \text { Fibrosis } & 19\end{array}$

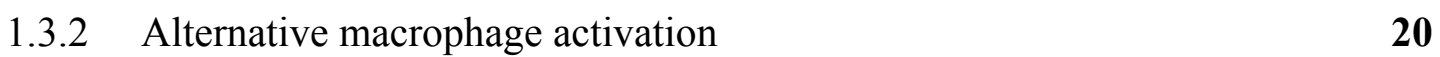

$\begin{array}{ll}\text { 1.3.3 Pneumonia } & \mathbf{2 3}\end{array}$

1.4 General aims and hypotheses $\quad 26$

CHAPTER 2 29

$\begin{array}{ll}\text { MATERIALS AND METHODS } & 29\end{array}$

$2.1 \quad$ Materials 29

2.1.1 Antibodies $\quad 29$

2.1.2 General chemicals and tissue culture reagents 30

2.1.3 Small interfering RNA (siRNA) 30

2.1.4 Primers

2.1.5 Animals $\quad \mathbf{3 4}$

$\begin{array}{lll}2.2 & \text { Tissue culture } & 34\end{array}$

2.3 SDS-PAGE and Western blotting 37

2.3.1 Cell lysis $\quad 37$

2.3.2 SDS-PAGE and Western blotting 37

$\begin{array}{lll}2.3 .3 & \text { Buffers } & 38\end{array}$ 
2.4 Protein purification $\quad 39$

2.4.1 Bacterial expression system $\quad 39$

2.4.2 Baculovirus expression system $\mathbf{4 2}$

2.5 Immunohistochemistry (IHC) 4

2.6 Quantitation of IHC using Openlab 44

$\begin{array}{lll}2.7 & \text { HSC isolation } & 45\end{array}$

$\begin{array}{lll}2.8 & \text { Immunofluorescence (IMF) } & 47\end{array}$

$\begin{array}{lll}2.9 & \text { SiRNA transfections } & 47\end{array}$

2.10 Real-time RT-PCR

2.10.1 RNA extractions $\quad \mathbf{4 8}$

2.10.2 RNA extraction from whole liver and kidney $4 \mathbf{4 9}$

2.10.3 DNase treatment of RNA

2.10.4 PCR to check for DNA contamination 49

2.10.5 Real-time RT-PCR

2.11 MTT assay

2.12 Cytokine assay $\quad 52$

2.13 Nitrite assay $\quad 53$

2.14 Arginase assay $\quad 54$

2.15 Antibacterial assay $\quad 54$

2.16 FITC-labelling of bacteria $\quad 55$

2.17 Trypan blue viability assay $\quad 55$

2.18 Preparation of human neutrophils from peripheral blood 56

2.19 Phagocytosis assays $\quad 56$

2.20 Neutrophil activation assays $\quad \mathbf{5 8}$

2.20.1 Neutrophil adhesion molecule expression $\quad \mathbf{5 8}$

2.20.2 Superoxide anion generation assay $\quad \mathbf{5 9}$

2.20.3 Dihydrorhodamine (DHR) activation $\quad \mathbf{5 9}$

2.21 In vivo animal models $\quad 60$

2.21.1 Chronic carbon tetrachloride $\left(\mathrm{CCl}_{4}\right)$ - induced liver injury model $\quad \mathbf{6 0}$

2.21.2 Model of kidney fibrosis using unilateral ureteric obstruction (UUO) $\quad \mathbf{6 0}$

2.21 .3 Pneumonia model $\quad 61$

2.22 Statistical analysis $\quad 62$

$\begin{array}{lr}\text { CHAPTER } 3 & 64\end{array}$

PURIFICATION OF GALECTIN-3 USING THE BACULOVIRUS EXPRESSION SYSTEM 64

$\begin{array}{lll}3.1 & \text { Introduction } & 64\end{array}$

$\begin{array}{lll}\text { 3.2 Methods and results } & 67\end{array}$

3.2.1 Generating the recombinant pFastBac vector $\quad 67$

3.2.2 Transformation of the E. coli strain TOP10 with recombinant vector $\quad \mathbf{7 2}$

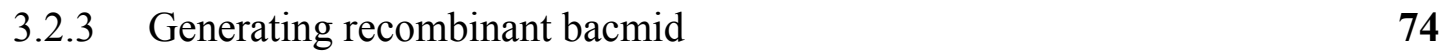

3.2.4 Analysing recombinant bacmid DNA by PCR $\quad \mathbf{7 5}$

$\begin{array}{ll}\text { 3.2.5 Transfecting insect cells with recombinant bacmids } & \mathbf{7 7}\end{array}$

$\begin{array}{ll}\text { 3.2.6 Isolating and amplifying baculoviral stocks } & \mathbf{7 8}\end{array}$

$\begin{array}{ll}3.2 .7 & \text { Expressing recombinant protein }\end{array}$

$\begin{array}{lll}3.3 & \text { Discussion } & 82\end{array}$ 
GALECTIN-3 PLAYS A CRITICAL ROLE IN ORGAN FIBROSIS $\quad \mathbf{8 3}$

$\begin{array}{lll}4.1 & \text { Introduction } & 83\end{array}$

$\begin{array}{lll}4.2 & \text { Results } & 83\end{array}$

4.2.1 Galectin-3 expression is upregulated in human liver fibrosis $\quad \mathbf{8 7}$

4.2.2 Galectin-3 is temporally and spatially related to fibrosis in a reversible $\quad \mathbf{8 7}$ rat model of liver fibrosis

4.2.3 Galectin-3 plays a critical role in liver fibrosis $\quad \mathbf{9 0}$

4.2.4 Myofibroblast activation is galectin-3 dependent 96

4.2.5 Disruption of the galectin-3 gene does not affect TGF- $\beta$ expression and $\mathbf{1 0 2}$ Smad signalling

4.2.6 Galectin-3 does not signal via the MEK/ERK pathway to exert its $\mathbf{1 0 8}$

effects in this model of liver fibrosis

4.2.7 Galectin-3 plays a critical role in kidney fibrosis $\quad \mathbf{1 1 1}$

$\begin{array}{lll}4.3 & \text { Discussion } & 116\end{array}$

$\begin{array}{lr}\text { CHAPTER 5 } & 119\end{array}$

REGULATION OF ALTERNATIVE MACROPHAGE ACTIVATION BY GALECTIN-3

$\begin{array}{llr}5.1 & \text { Introduction } & 119\end{array}$

$\begin{array}{llr}5.2 & \text { Results } & 122\end{array}$

5.2.1 Disruption of the galectin-3 gene in macrophages causes a specific $\quad \mathbf{1 2 2}$

defect in alternative activation of in vitro and in vivo differentiated

macrophages

5.2.2 IL-4 stimulated alternative macrophage activation is dependent on $\mathbf{1 3 5}$ expression of galectin-3 and CD98 and activation of PI3-K

5.2.3 Galectin- $3^{-/-}$mice show reduced expression of the alternative $\quad 144$ macrophage markers compared to wild type mice following UUO

$\begin{array}{lll}5.3 & \text { Discussion } & 147\end{array}$

CHAPTER 6

$\begin{array}{lr}\text { GALECTIN-3 AND PNEUMONIA } & 153\end{array}$

$\begin{array}{lll}6.1 & \text { Introduction } & 153\end{array}$

$\begin{array}{llr}\text { 6.2 Results } & 155\end{array}$

6.2.1 In vivo studies $\quad \mathbf{1 5 5}$

6.2.2 Galectin-3 plays a role in the clearance of $S$. $p n$ from the lungs and $\mathbf{1 5 6}$

protects the lung from damage

6.2.3 Galectin-3 does not play a significant role in the clearance of E. coli 159 pneumonia

6.2.4 Galectin-3 is bactericidal against $S$. pn at high concentrations $\quad \mathbf{1 6 2}$

6.2.5 Galectin- $3^{-/-}$macrophages produce less nitrite, compared to wild type, $\mathbf{1 6 2}$

in response to both $S$. pn and E. coli in vitro 
6.2.6 Galectin- $3^{-/-}$macrophages demonstrate similar levels of phagocytosis to wild type macrophages

6.2.7 Phagocytosis of apoptotic human neutrophils by mouse BMDMs

6.2.8 Exogenously added recombinant galectin-3 causes human neutrophil activation and the generation of ROS

6.2.9 Purification of mouse bone marrow neutrophils

6.2.10 Apoptosis of mouse bone marrow derived neutrophils

6.2.11 Phagocytosis of apoptotic mouse neutrophils by mouse BMDMs

6.2.12 Phagocytosis of bacteria by mouse bone marrow neutrophils

6.2.13 Exogenously added recombinant galectin-3 activates both wild type and galectin- $3^{-/-}$mouse peripheral neutrophils

CHAPTER 7 


\section{List of Figures}

\section{CHAPTER 1}

\section{INTRODUCTION}

1.1 Classification of the mammalian galectin family

1.2 The effect of galectin-3 on immune cell function

1.3 Galectin-3 expression in wild type and galectin- $3^{-/-}$bone marrow

\section{CHAPTER 2}

MATERIALS AND METHODS

2.1 Map of pcDNA 3.1

\section{CHAPTER 3}

\section{PURIFICATION OF GALECTIN-3 USING THE BACULOVIRUS EXPRESSION}

\section{SYSTEM}

3.1 Schematic diagram illustrating the M13 primer sites on the

recombinant bacmid

3.2 Maps of the pFastBac HT vectors

3.3 Restriction digests of the recombinant pFastBac HT Vectors

3.4 Restriction digests of mini-preps from transformed TOP10 bacteria

3.5 PCR analysis of the recombinant bacmids

3.6 SDS-PAGE analysis of His-tagged recombinant mouse and human galectin-3

3.7 Western blot analysis of recombinant mouse and human galectin-3

81

\section{CHAPTER 4}

\section{GALECTIN-3 PLAYS A CRITICAL ROLE IN ORGAN FIBROSIS}

4.1 TGF- $\beta /$ Smad signalling pathway

4.2 Galectin-3 expression is upregulated in human liver fibrosis

4.3 Galectin-3 is temporally and spatially related to fibrosis in a reversible rat model of liver fibrosis

4.4 Galectin-3 plays a critical role in liver fibrosis

4.5 Myofibroblast activation is galectin-3 dependent

4.6 Galectin-3 siRNA inhibits myofibroblast activation and procollagen

(I) expression in HSCs

4.7 Galectin-3 regulates myofibroblast activation and hepatic fibrosis

103-107 despite similar levels of TGF- $\beta$ expression and signalling 
4.8 Galectin-3 does not signal via the MEK/ERK pathway to exert its

effects in this model of liver fibrosis

4.9 Disruption of the galectin-3 gene does not affect macrophage

112-113 recruitment following UUO

4.10 Absence of galectin-3 protects against renal fibrosis

114-115

CHAPTER 5

REGULATION OF ALTERNATIVE MACROPHAGE ACTIVATION BY GALECTIN-3

5.1 Disruption of the galectin-3 gene does not affect BMDM maturation 123

5.2 Alternative macrophage activation by IL-4 and IL-13 is abrogated in galectin- $3^{-/-}$BMDMs

5.3 IL-4 and IL-13 induced alternative activation is reduced in galectin$3^{-/-}$BMDMs

5.4 Galectin- $3^{-/-}$peritoneal and alveolar macrophages display normal

128-130 classical activation but impaired alternative activation

5.5 IL-4/IL-13 signalling via the JAK/STAT signalling pathway

132-134

5.6 Galectin-3 and CD98 expression increases in THP-1 cells following 136 differentiation into macrophage-like cells

5.7 Alternative activation of human macrophages requires galectin-3

140-143 and CD98

5.8 Reduced alternatively activated macrophages in galectin- $3^{-/-}$mouse

kidney following UUO

5.9 Proposed galectin-3 signalling mechanism for the alternative

146 activation of macrophages

\section{CHAPTER 6}

GALECTIN-3 AND PNEUMONIA

6.1 Galectin-3 plays a critical role in the clearance of an acute pneumococcal infection

6.2 Galectin-3 does not play a significant role in the clearance of an acute $E$. coli pneumonia infection

6.3 Antimicrobial activity of galectin-3 to $S$. pn

160-161

6.4 Galectin- $3^{-/-}$macrophages release less nitrite in response to both $S$. $p n$ and $E$. coli but not in response to IFN $\gamma /$ LPS

6.5 Galectin-3 is not required for effective phagocytosis of $S$. pn or $E$. coli by BMDM or alveolar macrophages

6.6 Galectin-3 may be required for effective phagocytosis of apoptotic human neutrophils by BMDM

6.7 Exogenously added recombinant galectin-3 primes fMLP-mediated superoxide release from human peripheral blood neutrophils

6.8 Exogenously added recombinant galectin-3 mediates L-selectin

(CD62L) shedding and CD11b upregulation on human peripheral blood neutrophils

168-172

174

176-178

180-181 
6.9 Purification of mouse bone marrow neutrophils

6.10 Apoptosis of WT and galectin- $3^{-/-}$mouse bone marrow derived neutrophils over 96 hours in culture

6.11 Phagocytosis of apoptotic mouse neutrophils by BMDM

6.12 Galectin-3 is not required for effective phagocytosis of $S$. $p n$ or $E$. coli by mouse neutrophils but enhances phagocytosis when added exogenously

6.13 Exogenously added recombinant galectin-3 increases CD11b expression on both wild type and galectin- $3^{-/-}$mouse whole blood neutrophils

6.14 Proposed model of pneumococcal pneumonia infection

197-198 


\section{Chapter 1}

\section{Introduction}

\section{$1.1 \quad$ Galectins}

\subsubsection{Background}

Animals and plants contain a large number of carbohydrate binding proteins called lectins (from the latin word legere meaning "to select"). Lectins are proteins which specifically bind or crosslink carbohydrates and initially consisted of two major families. The C-type family binds carbohydrates in a calcium dependent manner and the S-type family. All members are soluble and bind lactose and related saccharides (Lobsanov et al., 1993). The S-type family was so called because its first discovered member was dependent on sulfhydryl residues but it has now been designated a new name, the galectins. The first galectin was described in the mid 1970's by Teichberg et al. (Teichberg et al., 1975) from electric organ tissue of Electrophorus electricus (electric eel) and was given the name electrolectin. Waard et al. confirmed the finding of $\beta$-galactoside binding lectins in homogenates of mammalian tissues (de Waard et al., 1976).

\subsubsection{Origin of the term "galectin"}

The general name for galectins was proposed by Barondes et al. in 1994 (Barondes et al., 1994a). Before this proposal for a universal nomenclature, the galectins had been given many different names depending on the circumstances of their discovery. Barondes suggested that membership into the galectin family requires the fulfilment of two criteria: "affinity for $\beta$-galactosides and significant sequence similarity in the carbohydrate binding site" (Barondes et al., 1994a). Over time it was realised that some of these proteins discovered from different species had very conserved structure-function relationships and so they were grouped and numbered sequentially according to Barondes' proposal (Barondes et al., 1994a). 
Table 1.1 lists four well characterised galectins and their original names before Barondes' proposal.

\begin{tabular}{|c|c|c|c|}
\hline Galectin & Original names & Molecular weight & Gene \\
\hline Galectin-1 & $\begin{array}{l}\text { L-14-I, L-14, RL-14.5, galaptin, } \\
\text { MGBP, GBP, BHL, CHA, HBP, HBL, } \\
\text { HLBP14, rIML-1, and other names }\end{array}$ & $14,500 \mathrm{Da}$ & LGALS1 \\
\hline Galectin-2 & L-14-II & $14,650 \mathrm{Da}$ & $L G A L S 2$ \\
\hline Galectin-3 & $\begin{array}{l}\text { CBP-35, Mac-2, IgEBP, CBP-30, RL- } \\
\text { 29, L-29, L-31, L-34, LBL, and other } \\
\text { names }\end{array}$ & $\begin{array}{l}\text { Between } 26,200 \\
\text { and } 30,300 \mathrm{Da}\end{array}$ & $L G A L S 3$ \\
\hline Galectin-4 & L-36, RIH & $36,300 \mathrm{Da}$ & LGALS4 \\
\hline
\end{tabular}

Table 1.1 - The proposed names for the well characterised mammalian galectins.

\subsubsection{The galectin family}

As mentioned above, galectins are defined to have affinity for $\beta$-galactoside containing glycoconjugates and have conserved sequence elements in the carbohydrate-binding site. The relevant amino acid residues that make up this sequence have been identified by X-ray crystallography (Lobsanov et al., 1993). Thus far, 15 members of the mammalian galectin family have been identified as well as galectins from many non-mammalian species and more are likely to be discovered. Hirabayashi and Kasai classified galectins based on their biochemical structure into three groups (Hirabayashi and Kasai, 1993); the Prototype galectins (galectins-1, -2, -5, -7, -10,-11, -13,-14 and -15), which contain one carbohydrate recognition domain (CRD) and a short $\mathrm{N}$-terminal sequence, exists as monomers or non-covalent homodimers; the Tandem Repeat group (galectin-4, -6, -8, -9, and -12) consist of two non-identical CRDs linked via a short peptide sequence; and the Chimera-type galectins (galectin-3), found in solution as monomers with an extended $\mathrm{N}$-terminal tail containing a proline-, glycine-, tyrosine-rich domain fused onto the CRD (figure 1.1). 
Figure 1.1 - Classification of the mammalian galectin family.
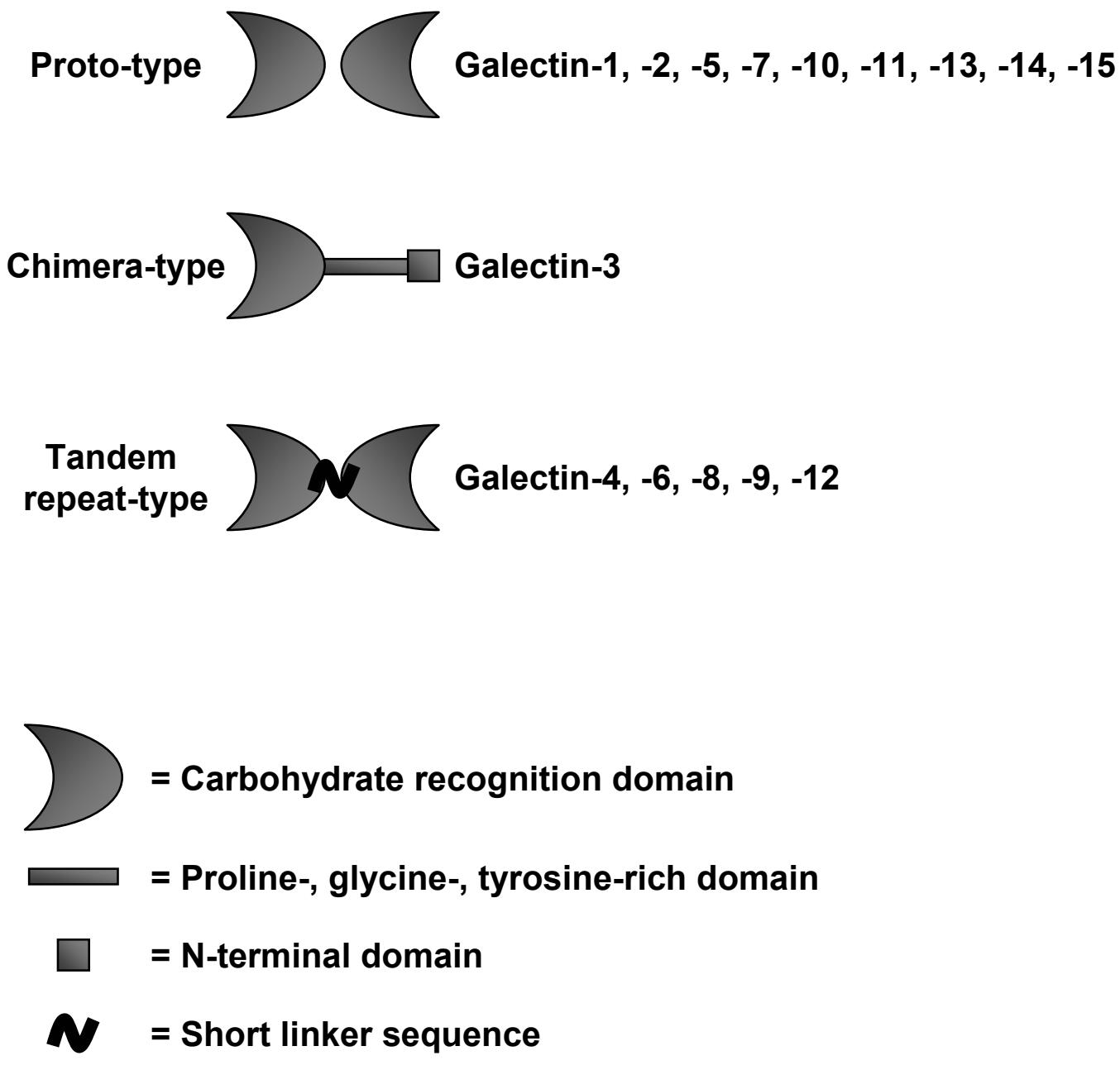

Figure 1.1

Schematic representation of the structure of different members of the galectin family (adapted from Rubinstein et al. 2004). 
Some galectins including the most characterised galectin-1 and -3 are found extracellularly as well as in the cytosol, however there is no evidence of a signal sequence classically used for externalisation through the endoplasmic reticulum/Golgi complex. Therefore these galectins must be secreted via a nonclassical pathway, which has yet to be elucidated. A partially characterised pathway of galectin-3 secretion suggests that this protein is concentrated in envaginations of the plasma membrane which pinch off and release galectin-3 into the extracellular space (Menon and Hughes, 1999; Gong et al., 1999; Hughes, 1999). The advantage of non-classical secretion is that the galectins are segregated from potential glycoprotein ligands traversing the classical pathway until they have exited from the cell where they can then carry out their functions (Hughes, 1999).

\section{$1.2 \quad$ Galectin-3}

\subsubsection{Distribution and localisation}

Galectin-3 is widely expressed in adult tissues. Particularly on activated macrophages and monocytes (Ho and Springer, 1982; Liu et al., 1995), basophils and mast cells (Liu, 1993), some epithelial cells (e.g. intestine and kidney) (Lotz et al., 1993; Castronovo et al., 1996; Bao and Hughes, 1995) and fibroblasts (Moutsatsos et al., 1987). Galectin-3 is also expressed in many tumours including thyroid and breast carcinomas where galectin-3 expression is inversely correlated with metastatic potential (Xu et al., 1995; Castronovo et al., 1996) and in colon carcinomas where the converse is seen, a direct correlation between galectin-3 expression and malignancy (Schoeppner et al., 1995). Intracellularly, galectin-3 is located in both cytoplasm and nucleus of some cells. In quiescent fibroblasts the distribution of galectin-3 is largely cytoplasmic, whereas in proliferating fibroblasts, the protein is located mainly in the nucleus, therefore distribution of galectin-3 in this cell type is coordinated with the proliferation state (Moutsatsos et al., 1987). Galectin-3 is upregulated when monocytes differentiate into macrophages (Liu et al., 1995) and down regulated when macrophages differentiate into dendritic cells (Dietz et al., 2000). 
Galectin-3 has numerous binding partners and demonstrates many autocrine and paracrine effects. It can affect several biological processes via the mediation of cell adhesion (Inohara and Raz, 1995; Kuwabara and Liu, 1996; Sato et al., 2002), cell activation (Liu et al., 1995; Yamaoka et al., 1995; Karlsson et al., 1998; Fernandez et al., 2005; Frigeri et al., 1993; Hsu et al., 1996) and chemoattraction (Sano et al., 2000). Galectin-3 plays a role in the maintenance of cellular homeostasis by affecting cell growth (Inohara et al., 1998; Joo et al., 2001; Yang et al., 1996), apoptotis (Hsu et al., 2000; Akahani et al., 1997; Yang et al., 1996; Fukumori et al., 2003) and controlling the cell cycle (Kim et al., 1999; Lin et al., 2000) and during immune responses (Hsu et al., 2000; Colnot et al., 1998b; Chen et al., 2005), oncogenesis and tumour invasion and metastasis (van den et al., 2004; Takenaka et al., 2004; Iurisci et al., 2000; Buttery et al., 2004; Kim et al., 1999).

\subsubsection{Regulation of galectin-3 expression}

The regulation of galectin-3 is a complex mechanism that involves numerous transcription factors and signalling pathways, and which depends on cell type, external stimuli and environmental conditions.

The gene for human galectin-3 LGALS3 is located on chromosome 14, locus q21-q22 (Raimond et al., 1997). The human and mouse LGALS3 genes are composed of six exons and five introns. Exon I and part of exon II encodes the untranslated region upstream of the translation initiation site located on exon II. Exon III of both the mouse and human LGALS3 gene encodes the N-terminal domain. In mouse, the CRD is encoded by exons IV, V and VI (Gritzmacher et al., 1992), whereas in human the sequence encoding the CRD was found entirely within exon V (Kadrofske et al., 1998).

The expression of galectin-3, on both transcription and translational levels, is affected by various stimuli. Galectin-3 protein and mRNA levels are increased in proliferating fibroblasts compared to quiescent cells (Moutsatsos et al., 1987; Agrwal et al., 1989). Galectin-3 can be considered a differentiation marker - galectin-3 is upregulated when monocytes differentiate into macrophages (Liu et al., 1995) and down-regulated when macrophages differentiate into dendritic cells (Dietz et al., 2000). Galectin-3 is also regarded as a macrophage activation marker due to the fact 
that galectin-3 is upregulated by the activation of the monocytic THP-1 cell line by phorbol ester (Dabelic et al., 2005) and in macrophages exposed to granulocytemacrophage colony stimulating factor (GM-CSF) (Saada et al., 1996).

Despite a large body of data concerning galectin-3 expression, the mechanisms of regulation of galectin-3 expression are still poorly understood. Neither the human, nor the murine LGALS3 promoter contains a TATA box immediately upstream of the transcription start site. However, there are multiple GC box motifs for binding of the ubiquitously expressed Sp1 transcription factor which is a common feature of constitutively expressed, or so-called housekeeping, genes (Kadrofske et al., 1998).

In addition to five putitive Sp1 binding sites, the promoter region of the human LGALS3 gene contains several regulatory elements: five cAMP-dependent response elements (CRE), four AP-1- and one AP-4-like sites, two NF- $\kappa \mathrm{B}-$ like sites, one sis-inducible element (SIE) and a consensus basic helix-loop-helix (bHLH) core sequence. Although the LGALS3 promoter looks like that of a housekeeping gene, galectin-3 expression is increased in response to serum stimulation and can be characterised as an immediate-early gene. The SIE is important for growth factorinduced transcriptional activation of other immediate-early genes and it has been suggested that SIE is a possible candidate for the growth-induced activation of LGALS3 expression, caused by the addition of serum (Saada et al., 1996).

The presence of CRE and NF- $\mathrm{KB}$-like sites in the promoter region implies that activation of galectin-3 expression could be regulated through signalling pathways involving the cAMP-response element-binding protein (CREB) or the NF$\kappa \mathrm{B}$ transcription factor. The involvement of NF- $\mathrm{B}$ transcription factor and the Jun protein, a component of AP-1 transcription factor, in the regulation of galectin-3 expression has been confirmed (Dumic et al., 2000; Kim et al., 2003).

\subsubsection{Intracellular binding partners, functions and signalling}

Intracellular binding partners for galectin-3 include Bcl-2 (Yang et al., 1996; Akahani et al., 1997), Gemin4 (Park et al., 2001), CBP70 (Seve et al., 1993), cystidine/histidine-rich protein (Chrp) (Menon et al., 2000), cytokeratins (Goletz et al., 1997) and $\beta$-catenin (Shimura et al., 2004). The majority of intracellular ligands, 
with the exception of cytokeratins, interact with galectin-3 via protein-protein rather than lectin-glycoconjugate interactions.

Gemin4 is a component of the spliceosome, a macromolecular complex that coordinates pre-mRNA splicing, which is an important post-transcriptional process. Galectin-3 is an interacting partner of Gemin4 and this interaction has been implicated as an important event in spliceosome assembly (Park et al., 2001).

Bcl-2 was the first cytosolic ligand of galectin-3 to be discovered. Galectin-3 has significant sequence similarity with the anti-apoptotic protein $\mathrm{Bcl}-2$, especially in that the lectin contains the NWGR motif conserved amongst the Bcl-2 family. Studies have shown that galectin-3 binds Bcl-2 and this interaction can be inhibited by lactose. This was an interesting finding as Bcl-2 is not a glycoprotein. The CRD of galectin-3 could be involved in this molecular interaction, or binding of lactose to galectin-3 may induce a conformational change in the region of the protein that does interact with Bcl-2. Together these results imply that galectin-3 plays a role in cell growth and apoptosis (Yang et al., 1996; Akahani et al., 1997). Other galectin-3 ligands involved in apoptotic signalling have recently been identified; CD95 (APO$1 /$ Fas) is a member of the death receptor family (Fukumori et al., 2004), Nucling is involved in the regulation of apoptosis (Liu et al., 2004) and Alix/AIP1 is also involved in the regulation of apoptotic events (Liu et al., 2002).

Galectin-3 has been shown to elevate Protein Kinase B (Akt/PKB) levels, a Ser/Thr protein kinase involved in regulating the inhibition of apoptosis and the stimulation of cell proliferation (Oka et al., 2005); further evidence linking galectin-3 with cancer progression.

Ras proteins are important small GTPases, playing a role in cell proliferation, differentiation, survival and death. Galectin-3 is a specific binding partner of activated K-Ras but does not interact with activated H-Ras or N-Ras (Elad-Sfadia et al., 2004). This specific binding promotes the activation of phosphatidylinositol 3kinase (PI3-K) and Raf-1 and augments extracellular signal-regulated kinase (ERK) activation. Galectin-1, on the other hand, binds to both activated H-Ras and K-Ras thus deflecting the Ras signal towards Raf-1 and away from PI3-K. K-Ras is the most important Ras oncoprotein in human tumours and as galectin-1 and galectin-3 
levels vary amongst normal and cancer cells it is possible to conclude that the balance of these galectins defines the outcome of oncogenic K-Ras transformation.

Galectin-3 has also been shown as an important regulator of Wnt/ $\beta$-catenin signalling. Galectin-3 has structural similarity to and is a binding partner of $\beta$ catenin. $\beta$-catenin is a downstream component of the Wnt signalling pathway. Phosphorylation of $\beta$-catenin by casein kinase I (CKI) and glycogen synthase kinase$3 \beta$ (GSK-3 $\beta$ ) is essential for signal initiation. Galectin-3 can also be phosphorylated by this dual kinase system, however, it is unclear whether phosphorylation of galectin-3 affects the phosphorylation of $\beta$-catenin or vice versa, or of the exact role galectin-3 plays during Wnt signalling (Shimura et al., 2004; Shimura et al., 2005). $\beta$-catenin signalling regulates TGF- $\beta$-mediated epithelial to mesenchymal transition (EMT), a process which generates myofibroblasts and contributes to the progression of fibrosis. Furthermore, Wnt4 upregulation in murine models of renal tubulointerstitial disease correlates with the appearance of fibrotic lesions thus implicating these proteins as mediators of renal fibrosis (Surendran et al., 2002).

Apical sorting is the process whereby proteins are directed to either the apical or basolateral domain of epithelial cells to maintain cellular polarisation. The apical domains of epithelial cells face the organ lumen and the basolateral domains face neighbouring cells and the basal lamina (Delacour and Jacob, 2006). Galectin-3 is found in lipid-raft-independent apical carrier vesicles and interacts with proteins bound for the apical domain in a glycan-dependent manner (Delacour et al., 2007). In galectin-3-depleted cells, the glycoproteins are misguided to the basolateral membrane. Furthermore, galectin-3 causes glycoprotein clustering, and in glycosylation-deficient cells cluster formation and apical sorting are restrained. Thus crosslinking glycoproteins by galectin-3 is required for apical sorting.

\subsubsection{Extracellular functions}

Once outside the cell, galectins interact with various cell surface $\beta$ galactosides containing glycans via the CRD. In vivo galectin-3 binds to ligands containing lactose as the basic unit of recognition (Bachhawat-Sikder et al., 2001). In general, galectins bind to Type I Gal $\beta 1,3$ GlcNAc or Type II Gal $\beta 1,4$ GlcNAc units with higher affinity to polylactosamine chains (Ochieng et al., 2004; Hughes, 
2001). Expression of these glycan structures depends on the activity of glycosyltransferases. $\quad \beta 1,6-\mathrm{N}$-acetylglucosaminyl transferase (Mgat5, GnT-V) promotes the addition of $\mathrm{N}$-acelyllactosamine on $\mathrm{N}$-glycans, thus creating the preferred ligands for galectin-3 (Dumic et al., 2006).

The majority of extracellular functions and subsequent signal transduction of galectin-3 are thought to be due to the CRD binding to Mgat5-modified N-glycans on various cell surface receptors. This is followed by the galectin forming oligomers via its N-terminal domain, thus functioning as a biological cross-linker among several glycoproteins. Previous data has shown that a truncated form of galectin-3 lacking the N-terminal domain, while still able to bind ligands, can not exert its functions (Kuwabara and Liu, 1996; Nieminen et al., 2005; Sato et al., 2002). Recent data has reinforced this proposal by demonstrating oligomerisation of galectin-3 at a cellular level in biological settings (Nieminen et al., 2007).

Integrins are highly glycosylated due to multiple glycosylation sites and galectin-3 is a binding partner of $\alpha 3 \beta 1$ integrin on the cell surface of endothelial cells. A complex is formed between the integrin, galectin-3 and the NG2 proteoglycan which may potentiate transmembrane signalling responsible for endothelial cell motility and morphogenesis (Fukushi et al., 2004). This interaction may be important in angiogenesis and tumour vascularisation.

Binding of galectin-3 to Mgat5-modified N-glycans of epidermal growth factor (EGF) and transforming growth factor (TGF)- $\beta$ receptors results in a delay of receptor removal by constitutive endocytosis. Therefore, the galectin-3 lattice ensures upregulation of surface receptors and increased sensitivity to growth factors. For example, Mgat5-deficient mice display a loss of sentivitity to cytokines resulting in a loss of phosphorylation and nuclear translocation of ERK and reduced Smad2/3 phosphorylation (Partridge et al., 2004).

Galectin-3 may therefore function to regulate cytokine receptors primarily through its ability to bind Mgat5-modified N-glycans on surface glycoproteins. There is no known catalytic activity of galectin-3 to directly induce downstream signalling pathways. 


\subsubsection{Cell adhesion}

Due to oligomerisation, galectin-3 can form multivalent aggregates suggesting that they act by crosslinking carbohydrate chains on cell surfaces and/or extracellular matrix (ECM) (Barondes et al., 1994b).

Extracellular binding partners of galectin-3 responsible for modulating cell adhesion include laminin (Ochieng and Warfield, 1995; Wang et al., 1992; Kuwabara and Liu, 1996; Massa et al., 1993), fibronectin and vitronectin (Matarrese et al., 2000; Sato and Hughes, 1992), hensin (Hikita et al., 2000), elastin (Ochieng et al., 1999) and collagen IV (Ochieng et al., 1998) as well as certain integrins including $\alpha 1 \beta 1$ integrins (Ochieng et al., 1998; Andre et al., 1999) and the $\alpha$ subunit of $\alpha \mathrm{M} \beta 1$ (CD11b/18, Mac-1 antigen) present on macrophages (Dong and Hughes, 1997). Galectin-3 also binds the heavy chain of CD98, promoting its dimerisation and, consequently, integrin activation (Dong and Hughes, 1997; Hughes, 2001; Dong and Hughes, 1996; Henderson et al., 2004).

Laminin, a key member of ECM proteins, is a heterotrimer that binds galectin-3 with high affinity (Massa et al., 1993) and this interaction is important in mediating cell adhesion. Because disturbed cell adhesion is an important characteristic of all cancers, the interaction between galectin-3 and laminin may be an important factor in cancer progression by, for example, protecting cells against detachment-induced apoptosis (Matarrese et al., 2000). Galectin-3 promotes adhesion of neutrophils to laminin (Kuwabara and Liu, 1996) which may aid neutrophils to traverse through basement membranes to reach the site of inflammation.

In general, galectin-3 positively regulates cell adhesion, however, it has been shown that galectin-3 can inhibit cell adhesion, for example, melanoma, breast cancer, fibrosarcoma and prostate cancer cell adhesion to laminin (Ochieng et al., 1998) thus increasing the possibility for cancers to metastasise. These differences rely on the proteins involved in cell adhesion and the concentration of soluble galectin-3 present. 


\subsubsection{Regulation of the immune response}

Galectin-3 is highly expressed and secreted from activated macrophages and acts as a powerful pro-inflammatory signal. As stated above, extracellular galectin-3 mediates cell adhesion, activation, and acts as a chemoattractant for various cell types. Numerous studies have been carried out investigating the effects of galectin-3 on the cells involved in immune responses (figure 1.2). Galectin-3 promotes the respiratory burst in neutrophils and monocytes (Yamaoka et al., 1995; Liu et al., 1995) and this activity is dependent on the lectin property of the protein as it is inhibitable by lactose; it induces mediator release from mast cells (Frigeri et al., 1993) and downregulates interleukin-5 (IL-5) production from eosinophils (Cortegano et al., 1998). Galectin-3 promotes the survival of B cells by blocking the final differentiation into plasma cells thus allowing the rising of a memory $\mathrm{B}$ cell phenotype (Acosta-Rodriguez et al., 2004). This process is important when the host meets a pathogen which it has previously encountered. Memory B cells enable the immune system to eliminate the secondary infection quicker and more effectively.

A good deal of research has been carried out investigating the effect of galectin-3 on T lymphocytes. Galectin-3 is expressed in activated, but not resting $\mathrm{T}$ lymphocytes (Joo et al., 2001). Extracellular galectin-3 induces T cell apoptosis (Fukumori et al., 2003) whereas intracellular galectin-3 results in an inhibition of apoptosis (Yang et al., 1996). Over expressing galectin-3 in the human leukaemia T cell line Jurkat enhances proliferation and confers resistance to apoptosis induced by anti-Fas antibody and staurosporine (Yang et al., 1996). Furthermore, anti-sense oligonucleotides specific for murine galectin-3 inhibit the proliferation of activated $\mathrm{T}$ lymphocytes (Joo et al., 2001). Inhibition of galectin-3 reduces naïve T-lymphocytedendritic cell interactions (Swarte et al., 1998), a process crucial for the induction of immune responses. Finally, Mgat5-dependent association of galectin-3 with T cell receptor (TCR) complex proteins restricts TCR recruitment to the site of antigen presentation thus inhibiting TCR-mediated signalling (Demetriou et al., 2001).

Regarding the influence of galectin-3 on neutrophil physiology, this protein increases CD66 expression on the surface of neutrophils (Fernandez et al., 2005) and binds to CD66a and CD66b (Feuk-Lagerstedt et al., 1999), the receptors most likely responsible for inducing neutrophil NADPH-oxidase activation. Galectin-3 
promotes the adhesion of neutrophils to laminin (Kuwabara and Liu, 1996), the extravasation of neutrophils in response to a streptococcal pneumonia but not an Escherichia coli (E. coli) infection (Sato et al., 2002) and enhances phagocytic activity (Fernandez et al., 2005).

Monocyte and macrophage biology is also affected by galectin-3. In addition to enhancing superoxide anion production from human monocytes (Liu et al., 1995), galectin-3 acts as a chemoattractant for monocytes and macrophages (Sano et al., 2000). At high concentrations the protein is chemotactic, that is to say cell migration is directed. At low concentrations galectin-3 is chemokinetic resulting in enhanced general motility. Galectin-3 positive macrophages show increased phagocytosis of Immunoglobulin-G (IgG)-opsonised erythrocytes and apoptotic thymocytes both in vitro and in vivo when compared to macrophages from galectin-3 knockout (galectin$3^{-/}$) mice (Sano et al., 2003). Furthermore, peritoneal macrophages taken from galectin- $3^{-/-}$mice are more prone to undergo apoptosis than wild type macrophages (Hsu et al., 2000). Galectin-3 also promotes monocyte-monocyte interactions that ultimately lead to polykaryon (multinucleated giant cell) formation, a phenotype associated with chronic inflammatory and fibrotic diseases (Okamoto et al., 2003).

The aforementioned effect on the different cells of the immune system signifies that galectin-3 must play an important role in certain disease states. Intracellular galectin-3 could promote the survival of inflammatory cells resulting in persistence of inflammation confirmed by the finding that galectin- $3^{-/-}$mice demonstrate a reduced inflammatory response after the induction of peritonitis (Colnot et al., 1998b). Galectin-3 recognises galactoside-containing glycoconjugates on pathogens and can bind to lipopolysaccharides (LPS) of a number of gramnegative bacteria (Mandrell et al., 1994) implicating the importance of galectin-3 in pathogen recognition. While endogenous galectin-3 is shown to play a role in the pathogenesis of asthma (Zuberi et al., 2004), galectin-3 applied therapeutically as gene therapy suppresses the inflammatory response in a rat asthma model (del, V et al., 2002) thus presenting novel therapeutic approach to the treatment of asthma. 
Figure 1.2 - The effect of galectin-3 on immune cell function.
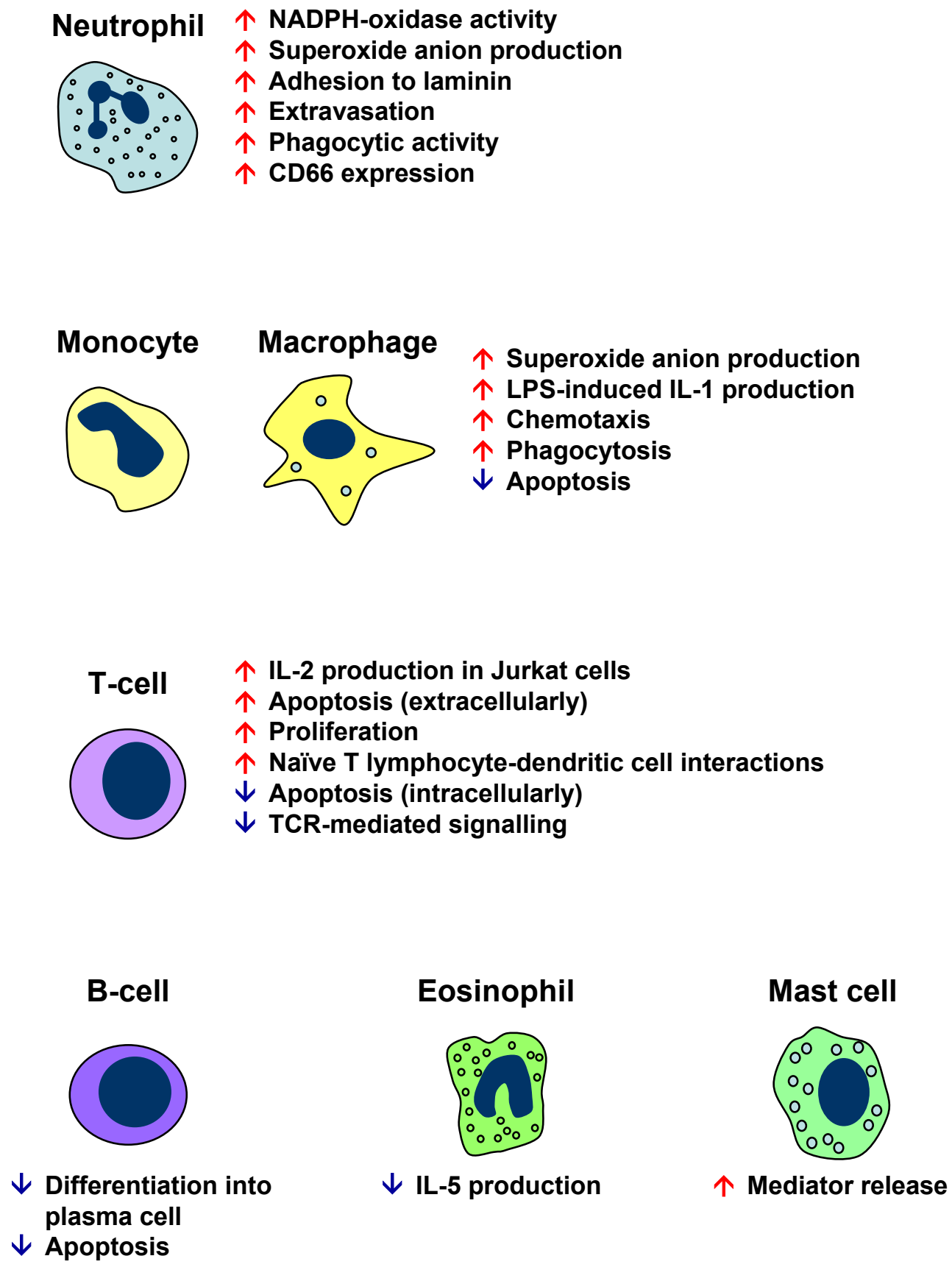

Figure 1.2

The effects of galectin-3 on immune cells. Red upwards arrows indicate positive effects, blue downwards arrows indicate negative effects. 
Investigating galectin-3 in the immune response has demonstrated that this protein displays both pro- and anti-inflammatory roles depending on the target cell type, whether galectin-3 is acting exogenously or endogenously, its expression level and other inflammatory factors. It is therefore imperative that all these factors be taken into consideration should galectin-3 be used therapeutically.

\subsubsection{Galectin-3 and cancer}

Galectin-3 expression in cancer has been widely studied, however there are conflicting results which makes it difficult to come to a general conclusion about the expression profiles of galectin-3 in cancer. The functional properties of galectin-3 mentioned above have, however, convinced researchers in this field that galectin-3 plays several roles in cancer pathogenesis, proliferation and metastasis. Furthermore, some progress has been made in developing galectin-3 inhibitors as anti-cancer agents (Cumpstey et al., 2005; Pieters, 2006).

Initial studies inferred that galectin-3 expression in cancer was increased and directly correlated with metastatic potential. Galectin-3 expression has been shown to be increased in a mouse fibrosarcoma cell line derived from a parent fibrosarcoma tumour induced by chronic UV irradiation (Raz et al., 1987). Galectin-3 expression is also increased in thyroid malignancies (Xu et al., 1995), gastric cancer (Baldus et al., 2000), tumours of the central nervous system (Bresalier et al., 1997), human bladder carcinomas (Cindolo et al., 1999), pancreatic tumours (Schaffert et al., 1998) and renal carcinoma (Young et al., 2001). Human colon cancer specimens demonstrate a positive correlation between the stage of progression of colorectal carcinomas and galectin-3 expression (Irimura et al., 1991). In contrast, another group demonstrated decreased expression of galectin-3 in colon cancer specimens compared to corresponding normal tissue (Castronovo et al., 1992). Furthermore, similar results have been obtained with skin (Konstantinov et al., 1994), head and neck (Choufani et al., 1999) and prostate cancer (Pacis et al., 2000; Ellerhorst et al., 1999). Galectin-3 is highly expressed in non-small cell lung cancer (NSCLC) yet very poorly expressed in small cell lung cancer (SCLC) which may be linked with variations in cellular behaviour (Buttery et al., 2004). It has been suggested that the 
apparent discrepancies in data could be due to different experimental approaches and techniques used and high specificity of each type of cancer (Dumic et al., 2006).

The intracellular distribution of galectin-3 determines its effect on cancers. Nuclear galectin-3 has anti-tumour effects whereas cytoplasmic galectin-3 is associated with tumour progression. Neoplastic progression of colon carcinoma has been shown to be associated with increased cytoplasmic galectin-3 expression and loss of nuclear galectin-3 expression (Lotz et al., 1993). Similar results have been shown with tongue (Honjo et al., 2000), prostate (Van Den Brule et al., 2000; Califice et al., 2004) and NSCLC (Puglisi et al., 2004). In contrast, galectin-3 is highly expressed in the nuclear compartment of transformed thyroid cells (Paron et al., 2003).

Examining the expression levels and distribution of galectin-3 in cancers could act as a prognostic tool for the diagnosis of cancer severity. This has particularly been shown for thyroid carcinoma whereby using galectin-3 to diagnose follicular carcinoma is over 95\% sensitive and specific (Volante et al., 2004).

Metastasis of cancer is a fatal complication associated with malignancies. The metastatic cascade involves angiogenesis at the primary site, detachment from the primary site, invasion through the ECM, dissemination through the blood flow, tumour cell aggregation in capillaries, extravasation and growth at secondary sites. Galectin-3 is involved in tumour related angiogenesis (Nangia-Makker et al., 2000); inhibits or promotes cell adhesion as detailed previously; protects cells from anoikis (Kim et al., 1999); increases tumour cell aggregation (Inohara and Raz, 1995) and contributes to tumour cell extravasation (Glinsky et al., 2001) - all of which add to the metastatic potential of cancers.

\subsubsection{CD98}

Previously known as 4F2, CD98 is a disulfide-linked $125 \mathrm{kDa}$ heterodimeric type II transmembrane glycoprotein composed of a glycosylated $85 \mathrm{kDa}$ heavy chain (designated CD98) and a nonglycosylated 40kDa light chain (Verrey et al., 2004). CD98 was originally discovered as a T-cell activation antigen (Haynes et al., 1981) and has been implicated in a number of cellular processes including cell adhesion, giant cell formation and amino acid transport across cell membranes of various cell 
types (Deves and Boyd, 2000). CD98 is ubiquitously expressed in most tissues and tumour cells (Parmacek et al., 1989) and is highly conserved between species (Quackenbush et al., 1987). It is expressed at low levels on the surface of quiescent cells but is rapidly upregulated following cellular activation.

Previous work from this laboratory has shown that CD98 promotes "integrinlike signalling" and PI3-K activation causing elevation of intracellular $\mathrm{PI}(3,4,5) \mathrm{P}_{3}$ and PKB activation leading to cellular transformation (Rintoul et al., 2002; Henderson et al., 2004).

Galectin-3 binds to the heavy chain of CD98 promoting its dimerisation and integrin activation (Dong and Hughes, 1997; Hughes, 2001; Dong and Hughes, 1996). Crosslinking CD98 with anti-CD98 monoclonal antibodies promotes polykaryon (multinucleated giant cell) formation, a phenotype associated with chronic inflammatory conditions (Tajima et al., 1999). Therefore modulation of galectin-3 expression and interaction with CD98 during macrophage differentiation may be important in the regulation of macrophage plasticity and control of macrophage fate. Activation of CD98 by galectin-3 may therefore represent a mechanism whereby galectin-3 mediates its effects on macrophage function.

\subsubsection{The galectin $-3^{-/-}$mouse}

A considerable amount of work carried out in this study has utilised the galectin- $3^{-/-}$mouse. The galectin- $3^{-/-}$mouse was generated both by Dr Françoise Poirier's group at the Cochin Institute Paris, France (Colnot et al., 1998a) and by Professor Fu-Tong Liu's group at the University of California (Hsu et al., 2000). Colnot et al. demonstrate that their galectin- $3^{-/-}$mice do not manifest any obvious defect in implantation or early development. These mice appear healthy and have a normal lifespan. It was therefore concluded that galectin-3 does not play any essential role in fundamental physiological processes, however this only ruled out an absolute requirement for the protein. Furthermore, the galectin- $3^{-/-}$mouse demonstrates no reduced expression of galectin-1 (Colnot et al., 1998a). As a result it has been possible to utilise this mutant for studies on the role of galectin-3 in various processes. Positive or negative galectin-3 expression in the wild type mice or galectin- $3^{-/-}$mice respectively was routinely confirmed during this study. Figure 
1.3 shows a galectin-3 Western blot of wild type and galectin- $3^{-/-}$bone marrow derived macrophages (BMDM). Furthermore, regular checks on these mice using polymerase chain reaction (PCR) verify the absence of galectin-3 in galectin- $3^{-/}$ mice (data not shown).

Since being developed and made widely available, the galectin $-3^{-/-}$mouse has been used in a number of studies. Colnot et al. demonstrated that their galectin- $3^{-/-}$ mice have significantly less numbers of neutrophils in the peritoneal cavity 4 days after thioglycolate-induced peritonitis, indicating that galectin-3 plays a role in granulocyte maintenance during inflammation (Colnot et al., 1998b). Hsu et al. also demonstrated attenuated peritoneal inflammatory responses in their galectin- $3^{-/-}$mice following thioglycolate-induced peritonitis, however they did not verify Colnot's findings of a reduction of neutrophils in the galectin- $3^{-/-}$mouse peritoneal cavity (Hsu et al., 2000). Both groups, however, showed that macrophage numbers in galectin- $3^{-/-}$mouse peritoneal cavities were reduced following thioglycolate-induced peritonitis (Colnot et al., 1998b; Hsu et al., 2000). Together, these results reveal galectin-3 as an important pro-inflammatory molecule. 
Figure 1.3 - Galectin-3 expression in wild type and galectin$3^{-l-}$ bone marrow derived macrophages (BMDM).

Galectin-3

Actin

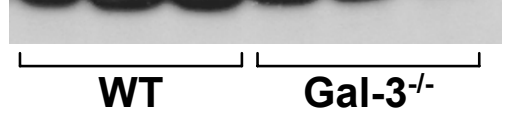

Figure 1.3

Galectin-3 Western blot of BMDM isolated from 3x WT and 3x galectin- $3^{-/-}$ mice. 


\subsection{Chronic inflammation and infection}

\subsubsection{Fibrosis}

Normal wound repair is tightly regulated by a sequence of events involving inflammation, recruitment, activation and proliferation of fibroblasts, and the production of ECM culminating in restoration of normal tissue architecture. This process is essential for the replacement of dead or damaged cells after injury and critical for survival (Wynn, 2007).

Following pathological chronic tissue injury platelets become activated, aggregate to form clots and degranulation from platelets causes an increase in blood vessel permeability. Leukocytes are recruited, activated and induced to proliferate by chemokines and growth factors such as platelet-derived growth factor (PDGF) and transforming growth factor (TGF- $\beta$ ). These activated leukocytes secrete profibrotic cytokines such as IL-13 and TGF- $\beta$. These profibrotic cytokines result in the activation of collagen-secreting, $\alpha$-smooth muscle actin ( $\alpha$-SMA) positive myofibroblasts resulting in the production of matrix metalloproteinases (MMPs) which disrupt the basement membrane. Activated myofibroblasts also secrete cytokines and chemokines that recruit more inflammatory cells to the site of injury. The source of myofibroblasts depends on the tissue and the nature of the injury. Myofibroblasts can be derived from resident tissue fibroblasts or from epithelial cells by a process of EMT (Hinz et al., 2007; Hinz, 2007; Zeisberg and Kalluri, 2004; Kalluri and Neilson, 2003). There is also mounting evidence that myofibroblasts can arise from circulating mesenchymal cells or fibrocytes (Bucala et al., 1994; Wang et al., 2007; Quan et al., 2006). Macrophages and neutrophils remove tissue debris and dead cells and any invading organisms. This initial inflammatory phase is followed by the production of ECM components by myofibroblasts and the formation of new blood vessels. Under normal conditions the myofibroblasts stimulate wound contraction with collagen fibres becoming more organised thus restoring the damaged tissue to its normal appearance. During chronic injury, whereby the inflammatory response persists for several weeks or months, inflammation, tissue destruction and repair processes occur simultaneously resulting in persistent 
myofibroblast activation and excessive ECM deposition forming a permanent fibrotic scar and loss of tissue architecture and function (Wynn, 2007).

\subsubsection{Alternative macrophage activation}

Macrophages play an important role in the regulation and orchestration of tissue responses following chronic inflammation. In particular, recent studies have demonstrated that macrophages display phenotypical heterogeneity in response to environmental factors that affect their role in inflammation.

The classically activated (M1 polarised) macrophage is the best studied macrophage phenotype and develops following an initial pro-inflammatory response during which $\mathrm{T}$ helper 1 (Th1)-type lymphocytes and natural killer (NK) cells produce interferon- $\gamma(\mathrm{IFN}-\gamma)$, and antigen presenting cells (APCs) produce an array of cytokines including IL-12 and IL-18 (Gordon, 2003). After an infection, LPS, a microbial trigger, classically activates macrophages following priming by IFN- $\gamma$. Once classically activated, macrophages produce the pro-inflammatory cytokines IL6 and tumour necrosis factor- $\alpha$ (TNF- $\alpha$ ) (Munder et al., 1998; Welch et al., 2002). They also produce nitrites including nitric oxide (NO) by an inducible nitric oxide synthase (NOS2) (Hauschildt et al., 1990; Hesse et al., 2001), which is important for microbial killing.

In contrast, the resolution phase of inflammation is driven by alternatively activated (M2 polarised) macrophages, which are hypo-responsive to proinflammatory stimuli. Macrophages undergo alternative activation when stimulated with the Th2 cytokines, IL-4 or IL-13 (Gordon, 2003; Munder et al., 1999; Hesse et al., 2001). Initially it was believed that IL-4 and IL-13 resulted in a deactivated phenotype similar to that seen with IL-10, whereby there is a deactivation of the respiratory burst and of inflammatory cytokine production, particularly TNF- $\alpha$. However, it was discovered that the regulatory role of IL-4 and IL-13 on immune responses appeared to be more complex than first thought (D'Andrea et al., 1995). 
Figure 1.4 - Classical (M1) and alternative (M2) activation of macrophages.

Classical (M1)

Alternative (M2)

IFN $\gamma$, TNF- $\alpha$, IL-1

IL-4, IL-13

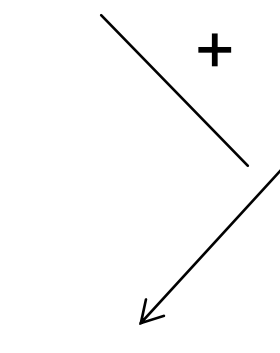

L-Hydroxy-

NOS2

arginine
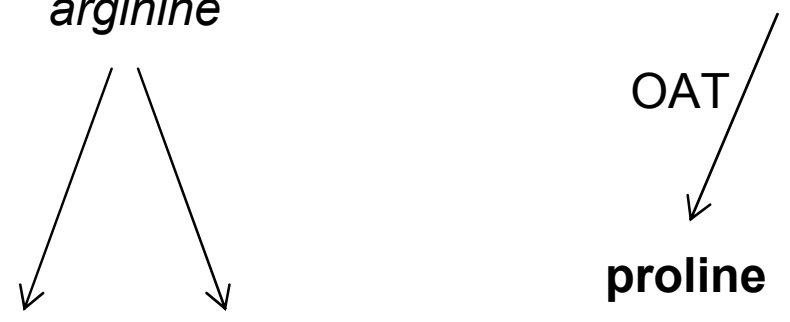

proline

urea

L-citrulline NO

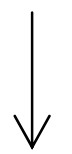

Collagen synthesis

Figure 1.4

Macrophages can be classically (M1) or alternatively (M2) activated as a result of competition between nitric oxide synthase 2 (NOS2) and arginase-1 for the common substrate L-arginine producing either nitric oxide (NO) or L-ornithine respectively. (OAT; ornithine aminotransferase, ODC; ornithine decarboxylase). 
Incubation of macrophages with IL-4 or IL-13 causes upregulation of mannose receptor expression (Stein et al., 1992), arginase-1 activity (Hesse et al., 2001), YM-1 (chitinase-like lectin) (Nair et al., 2005; Raes et al., 2002) and FIZZ-1 (found in inflammatory zone-1, a resistin-like secreted protein) expression (Herbert et al., 2004; Nair et al., 2003).

One mechanism by which Th1 and Th2 cytokines induce opposing macrophage phenotypes is through differential regulation of NOS2 or arginase-1 (Hesse et al., 2001) (Figure 1.4). Th1 cytokines IFN $\gamma$ and TNF- $\alpha$ cause macrophages to metabolise L-arginine by NOS2 to NO and L-citrulline. The intermediate product, L-hydroxyarginine, inhibits arginase-1 activity resulting in a classical (M1) phenotype. If Th2 cytokines IL-4 and IL-13 are present, less L-hydroxyarginine is produced and arginase-1 activity is not inhibited. Consequently, the common substrate L-arginine is metabolised by arginase- 1 resulting in the production of Lornithine and an alternative (M2) phenotype. L-ornithine is a necessary metabolite for the production of proline (a critical amino acid for the synthesis of collagen) which links arginase activity to fibrosis (Hesse et al., 2001; Misson et al., 2004; Endo et al., 2003).

\section{$\underline{\text { Alternative macrophage activation and Fibrosis }}$}

Arginase expression and activity is raised in the blood and airways of patients with fibrotic diseases (Grasemann et al., 2005; Grasemann et al., 2006; Mora et al., 2006) and in animal models of lung fibrosis (Hesse et al., 2001; Endo et al., 2003). Studies blocking arginase activity have demonstrated a direct role for the arginase biosynthetic pathway in pathological fibrosis (Liu et al., 2005). IL-4/IL-13 alternatively activated macrophages are hypothesised to serve as central regulators of fibrosis. A shift to M1 or classical macrophage activation by administration of IL-12 results in NOS2 expression and a reduction of fibrosis severity (Wynn et al., 1995). Co-culture of alternatively activated macrophages with fibroblasts increase the proliferation index and collagen synthesis of fibroblasts, suggesting that alternatively activated macrophages enhance fibrogenesis (Song et al., 2000). Recent work has demonstrated that selective depletion of macrophages in models of hepatic inflammation and progressive glomerulonephritis significantly attenuates fibrosis (Duffield et al., 2005b; Duffield et al., 2005a). However studies on murine silicosis 
suggest that increased expression of arginase-1 is not a sole determinant for the later development of lung fibrosis (Misson et al., 2004). Furthermore, macrophage/neutrophil specific knockout of the IL-4/IL-13 common receptor, IL$4 \mathrm{R} \alpha$, does not prevent collagen deposition and hepatic fibrosis in Schistosoma mansoni infected mice (Herbert et al., 2004). Thus, further work is required to define the precise biological role of alternative M2 macrophages in chronic inflammation and fibrosis and the mechanisms regulating macrophage polarisation in these disease states.

\section{Alternative macrophage activation and Infection}

Alternatively activated macrophages have been widely implicated in the progression of parasitic infections. It has been shown that the M2 macrophage contributes to the susceptibility of cutaneous leishmaniasis (Holscher et al., 2006) and that certain parasitic infections result in the generation of alternatively activated macrophages. Infection with the helminth parasite Fasciola hepatica induces a polarised Th2 immune response resulting in the alternative activation of macrophages (O'Neill et al., 2000; Donnelly et al., 2005). In these studies, IL-4 ${ }^{-/-}$ mice, which mounted a Th1 response, demonstrated the lowest level of liver damage. Similar results have been shown with other helminth infections including Brugia malayi and Schistosoma (Loke et al., 2000; Noel et al., 2004).

Some research into the role of alternatively activated macrophages during bacterial infections has been carried out in recent years. The presence of alternatively activated macrophages during progressive tuberculosis results in an inability to control bacterial replication (Kahnert et al., 2006). Alternative macrophage phenotype has also been observed in Whipple Disease, a rare systemic disease caused by the bacterium Tropheryma whipplei (Desnues et al., 2005). Alternatively activated macrophages, it has been suggested, contribute to the pathophysiological properties of this disease.

\subsubsection{Pneumonia}

Despite improvements in therapy and intensive-care support, mortality due to respiratory tract infections is ever increasing, the gram positive bacterium 
Streptococcal pneumoniae ( $S$. pn) being the major pathogen responsible. $S$. $p n$ is the most common cause of community acquired pneumonia (CAP). Mortality is high, especially in developing countries where pneumococcal pneumonia causes one million deaths per year in children under the age of 5 years (Paterson et al., 2005). Even with treatment, there is still an overall mortality rate of $23 \%$ among hospitalised patients (Bergeron et al., 1998; Dallaire et al., 2001). Along with pneumonia, the pneumococcus pathogen is the common bacterial agent in a wide range of infections including mucosal infections (sinusitis and otitis media), arthritis, pericarditis, peritonitis and the severe invasive infections meningitis and septicaemia (Obaro and Adegbola, 2002). Limitations of conventional therapies and emerging drug resistance amongst strains of $S$. $p n$ to antibiotics, such as penicillin (Feldman, 2004) and vancomycin (Novak et al., 1999), necessitates the continued study into the mechanisms involved in the pathogenesis of pneumococcal disease and the host immune defence against pneumococcal invasion.

Pneumonia caused by $S$. $p n$ is a consequence of failure by the host to clear or kill pneumococci inhaled into the lung (Gordon et al., 2000). Colonisation of the nasopharynx occurs in up to $40 \%$ of the population and aspiration of this small innoculum of bacteria is common (Dockrell et al., 2003). Most cases of pneumococcal pneumonia occur when the host is unable to overcome and eliminate these small challenges. Bergeron et al. (Bergeron et al., 1998) identified the essential steps that contribute to the pathogenesis of pneumococcal pneumonia beginning with partial but ineffective phagocytosis of bacteria by alveolar macrophages and the release of various pro-inflammatory cytokines in bronchoalveolar lavage (BAL) fluid (TNF and IL-6), lung tissues (IL-1 and IL-6) and serum (IL-6). Recruitment of neutrophils follows with further release of pro-inflammatory cytokines, active oxygen metabolites and defensins (Sibille and Reynolds, 1990). While these products kill the invading pathogens, they can also severely damage the lung tissue itself (Sibille and Reynolds, 1990). Tissue injuries become visible and more leukocytes migrate from the blood to the lungs. Alveolar architecture is destroyed with leakage of protein into the vasculature, unrestrained bacterial growth in both the lungs and the blood (bacteremia), loss of body weight and high mortality rate. 
However, successful clearance of an infection does occur in most individuals exposed to this small innoculum. Alveolar macrophages phagocytose bacteria that reach the terminal bronchioles and alveoli (Jonsson et al., 1985; Franke-Ullmann et al., 1996), and secrete cytokines and chemokines which recruit leukocytes to the site of infection. The recruited neutrophils also play an essential role in pneumococcal killing, and non-resident macrophages contribute to the resolution of inflammation by phagocytosing cell debris and apoptotic neutrophils. Therefore, the mechanisms that orchestrate macrophage and neutrophil recruitment/activation are important for the clearance of bacteria and resolution of inflammation.

\section{Phagocytosis}

Russian embryologist, Ilya Metchnikoff, popularised the idea of phagocytosis at the end of the $19^{\text {th }}$ century and hypothesised that phagocytosis could play an important role in host defence and tissue homeostasis (Metchnikoff 1905).

Distinct cell surface receptors, or "pattern recognition receptors", on phagocytes enable them to recognise and ingest foreign pathogens by recognising the particles directly or identifying targets coated with opsonic molecules.

Pathogens are recognised by a number of receptors on the alveolar macrophage cell surface including Fc receptors (which recognise the Fc component of associated immunoglobulin bound to bacteria), complement receptors (which recognise complement stabilised on bacterial surfaces), macrophage scavenger receptor, platelet-activating factor receptor, and CD14/toll-like receptor (TLR) complexes (Gordon et al., 2000).

Galectin-3 has been shown to have a critical role in phagocytosis of IgGopsonised erythrocytes and apoptotic thymocytes (Sano et al., 2003), however it's role in the phagocytic clearance of microorganisms and apoptotic neutrophils has not been elucidated. 


\subsection{General aims and hypotheses}

The aims of my $\mathrm{PhD}$ were to examine the role of galectin-3 in the development of fibrosis following chronic inflammation and in the response of macrophages and neutrophils to pneumococcal infection. This was carried out using the galectin- $3^{-/-}$mouse on a $129 \mathrm{~s} / \mathrm{v}$ background. Comparisons were made using strain and age matched control mice. In vitro experiments were used to further examine the role of galectin-3 during these disease states. In addition, this thesis describes, in detail, the process of purifying the galectin-3 protein used in numerous experiments.

\section{Hypothesis 1}

"Upregulation of galectin-3 expression during tissue fibrosis triggers hepatic stellate cell (HSC) activation and contributes to the pathogenesis of disease."

The scientific basis of this hypothesis derives from the following observations. Increased galectin-3 expression has been noted in tissue fibrosis (Hsu et al., 1999; Wang et al., 2000; Kasper and Hughes, 1996) and in vitro exogenous galectin-3 stimulates myofibroblast proliferation via the MEK1/2-ERK1/2 signalling pathway (Maeda et al., 2003). The relevance of these observations to the mechanistic role of galectin-3 in the pathogenesis of tissue fibrosis in vivo has not been examined.

The chronic carbon tetrachloride $\left(\mathrm{CCl}_{4}\right)$ model of liver fibrosis and the unilateral ureteric obstruction (UUO) model of kidney fibrosis in wild type and galectin- $3^{-/-}$ mice was used to test this hypothesis in vivo. The $\mathrm{CCl}_{4}$ rat model of liver fibrosis had previously been established by Professor John Iredale's group (University of Edinburgh) and subsequently applied to mice for this study. The UUO model of kidney fibrosis had been used previously by Dr Jeremy Hughes' group (University of Edinburgh) and was performed by Spike Clay. This model is a very attractive model for studying renal fibrosis as many of the pathophysiological events occur within one week post obstruction (Klahr and Morrissey, 2002). The model of UUO is 
neutrophil and lymphocyte independent and is characterised by marked tubulointerstitial macrophage infiltration together with progressive scarring (Diamond et al., 1995; Diamond, 1995). In vivo work was followed by in vitro studies of wild type and galectin- $3^{-/-}$HSC activation in response to galectin-3 and TGF- $\beta$.

\section{Hypothesis 2}

"Presence of galectin-3 and CD98 are necessary for alternative macrophage activation".

The scientific basis of this hypothesis derives from the following observations. Galectin-3 is highly expressed and secreted by macrophages and recent data suggests that galectin-3 plays a significant role in many facets of macrophage biology. Classical activation of macrophages by LPS has been shown to downregulate galectin-3 expression (Liu et al., 1995). Previous work from this laboratory has shown that CD98 promotes "integrin-like signalling" and PI3-K activation causing elevation of intracellular $\mathrm{PI}(3,4,5) \mathrm{P}_{3}$ and $\mathrm{PKB}$ activation leading to cellular transformation (Rintoul et al., 2002; Henderson et al., 2004). A number of studies suggest that PI3-K activation may be the final common pathway to alternative (M2) macrophage activation. The PI3-K/PKB pathway in macrophages negatively regulates NOS2 expression (Diaz-Guerra et al., 1999) and suppresses LPS-induced inflammation in endotoxaemic mice (Schabbauer et al., 2004).

The activation status of in vitro differentiated bone marrow derived macrophages (BMDMs) and in vivo differentiated peritoneal macrophages from wild type and galectin- $3^{-/-}$mice in response to Th1 or Th2 cytokines was assessed. Galectin- 3 and CD98 expression in the THP-1 macrophage-like cell line was manipulated to specifically assess the role of these proteins in alternative macrophage activation and signalling. 


\section{Hypothesis 3}

"Galectin-3 reduces the severity of pneumococcal pneumonia by augmenting immune cell function".

The scientific basis of this hypothesis derives from the following observations. Following pneumococcal infection of the lungs, galectin-3 accumulates in the alveolar space and this correlates with the onset of neutrophil extravasation (Sato et al., 2002). However, although neutrophils are actively recruited into Escherichia coli (E. coli) pneumonia infected lungs there is no increase in galectin-3 expression. Furthermore galectin-3 is released by alveolar macrophages upon incubation with Streptococcal pneumoniae (S. pn) membrane fraction (Sato et al., 2002). The relevance of these observations to the mechanistic role of galectin-3 in the host immune response to $S$. pneumoniae infection has not been examined.

The in vivo models of infection used in this study were chosen due to the presence of expertise in the Centre for Inflammation Research (University of Edinburgh) at intratracheal (i.t.) administration of substances to mice. Dr John Simpson's group assisted us in infecting our mice with $S$. $p n$ and $E$. coli and helped with the retrievals (details are given in Acknowledgements and Materials and Methods). The $S$. $p n$ and E. coli pneumonia models were chosen due to the findings by Sato et al mentioned above. In vivo work was followed by in vitro experimentation to study the role of galectin-3 on wild type and galectin- $3^{-/-}$macrophage and neutrophil activation and phagocytosis. 


\section{Chapter 2}

\section{Materials and Methods}

\section{$2.1 \quad$ Materials}

\subsubsection{Antibodies}

Antibodies were obtained from the following sources: anti-actin, anti- $\alpha$ smooth muscle actin ( $\alpha$-SMA) (clone 1A4), anti- $\alpha$-SMA-fluorescein isothiocyanate (FITC) (clone 1A4) and anti-MAP Kinase Activated (diphosphorylated extracellular signal-regulated kinase (ERK)-1\&2) (clone MAPK-YT) were from Sigma-Aldrich. Anti-Protein Kinase B (Akt/PKB) $\left[\mathrm{pS}^{473}\right]$ phosphospecific antibody (clone 14-6), anti-Jun N-terminal kinase (JNK) $1 \& 2\left[\mathrm{pTpY}^{183 / 185}\right]$ polyclonal phosphospecific antibody and anti-Smad-3 $\left[\mathrm{pSpS}^{423 / 425}\right]$ polyclonal phosphospecific antibody were from Biosource. Anti-arginase-1 (clone H-52), anti-human CD98 (clone C-20), antimouse CD98 (clone M-20) and anti-pSmad2/3 ( $\operatorname{Ser}^{433 / 435}$ ) were from Santa Cruz Biotechnology. Anti-mouse CD11b (clone 5C6), anti-mouse F4/80 (clone CI: A3-1), anti-mouse F4/80-FITC (clone CI: A3-1) and anti-human CD206 (mannose receptor) (clone 15-2) were from Serotec. Anti-mouse LY-6G and LY-6C-phycoerythrin (PE) (GR-1) (clone RB6-8C5), anti-human CD11b-allophycocyanin (APC) (clone ICRF44), anti-mouse CD11b-APC (clone M1/70), anti-mouse CD11b-PE (clone M1/70), anti-human CD16-FITC (clone 3G8), anti-human CD62L-PE (clone DREG56), FITC-mouse immunoglobulin G1 (IgG1) isotype control (clone MOPC-21), APC-mouse IgG1 isotype control (clone MOPC-21), PE-mouse IgG1 isotype control (clone MOPC-21) and APC-rat IgG2b isotype control (clone A95-1) were from BD Pharmingen. Anti-phospho-Protein Kinase C (PKC) (Pan) (betaII Ser660), antiAKT/PKB and anti-phospho-STAT-6 (Tyr641) were from Cell Signaling Technology. Anti-mouse Mac-2 (galectin-3) (clone M3/38) and anti-mouse Mac-2FITC (clone M3/38) were from Cedarlane. Anti-galectin-3 (clone A3A12) was from Affinity Bioreagents. Anti-human galectin-3 (clone 9C4) was from Novocastra. Anti-rat galectin-3 was kindly donated by FT Liu (University of California, Davis, 
USA). Anti-mouse YM-1 was kindly donated by Professor J Allen (University of Edinburgh, UK). All anti-species specific horseradish peroxidase (HRP)-conjugated antibodies were from DakoCytomation. All anti-species specific Alexa-Fluorconjugated antibodies were purchased from Invitrogen.

\subsubsection{General chemicals and tissue culture reagents}

Tissue culture plates and dishes were purchased from Corning Life Sciences. $15 \mathrm{ml}$ and $50 \mathrm{ml}$ conical tubes were purchased from BD Biosciences. Tissue culture reagents Dulbecco's Modified Eagle's Medium (DMEM), RPMI, Iscove's Modified DMEM (IMDM), Hanks' Balanced Salt Solution (HBSS), Dulbecco's Phosphate Buffered Saline (PBS), Foetal Bovine Serum (FBS), Foetal Calf Serum (FCS), penicillin/streptomycin and L-glutamine $(200 \mathrm{mM})$ were purchased from PAA (The Cell Culture Company). DMEM-F12 was purchased from Gibco.

Recombinant mouse and human Interleukin (IL)-4 and IL-13, interferon- $\gamma$ (IFN $\gamma$ ), lipopolysaccharide (LPS), recombinant mouse platelet-derived growth factor-BB (PDGF-BB) and recombinant transforming growth factor- $\beta$ (TGF- $\beta$ ) were purchased from R\&D. All cytokines were reconstituted as indicated in the datasheets, aliquotted to avoid repeated freeze-thaw cycles and stored at $-80^{\circ} \mathrm{C}$.

\subsubsection{Small interfering RNA (siRNA)}

Four siRNA duplexes against human and mouse galectin-3 and four duplexes against human CD98 were purchased from the Dharmacon siGENOME Collection of pre-designed siRNA reagents. siGENOME siRNA reagents are designed using Dharmacon's SMARTselection siRNA design algorithm to reduce off-target effects and decrease false positives. 5X siRNA buffer and nuclease-free $\mathrm{H}_{2} \mathrm{O}$ are provided within the package. Upon receipt the siRNA was reconstituted to a stock concentration using the recommended amount of 1X siRNA buffer (diluted from the $5 \mathrm{X}$ siRNA buffer with nuclease-free $\mathrm{H}_{2} \mathrm{O}$ ) according to the quantity of siRNA purchased. Once resuspended, the siRNA was stored in small aliquots at $-20^{\circ} \mathrm{C}$. 
The double stranded RNA oligomer sequences for mouse galectin-3 were:

\begin{tabular}{lll} 
Duplex & \multicolumn{2}{c}{ Sequence } \\
Duplex 1 & Sense & 5'-GAUGUUGCCUUCCACUUUAUU-3' \\
& Antisense & 5'-P.UAAAGUGGAAGGCAACAUCUU-3' \\
Duplex 2 & Sense & 5'-GCAGACAGCUUUUCGCUUAUU-3' \\
& Antisense & 5'-P.UAAGCGAAAAGCUGUCUGCUU-3' \\
Duplex 3 & Sense & 5'-GGUCAACGAUGCUCACCUAUU-3' \\
& Antisense & 5'-P.UAGGUGAGCAUCGUUGACCUU-3' \\
Duplex 4 & Sense & 5'-GGACAGGCUCCUCCUAGUGUU-3' \\
& Antisense & 5'-P.CACUAGGAGGAGCCUGUCCUU-3' \\
P represents phosphate &
\end{tabular}

The double stranded RNA oligomer sequences for human galectin-3 were:

\begin{tabular}{lll} 
Duplex & \multicolumn{2}{c}{ Sequence } \\
Duplex 1 & Sense & 5'-GAAGAAAGACAGUCGGUUUUU-3' \\
& Antisense & 5'-P.AAACCGACUGUCUUUCUUCUU-3' \\
Duplex 2 & $\begin{array}{l}\text { Sense } \\
\text { Antisense }\end{array}$ & 5'-GCAAUACAAAGCUGGAUAAUU-3' \\
& 5'-UUAUCCAGCUUUGUAUUGCUU-3' \\
Duplex 3 & Sense & 5'-GUACAAUCAUCGGGUUAAAUU-3' \\
& Antisense & 5'-P.UUUAACCCGAUGAUUGUACUU-3', \\
Duplex 4 & Sense & 5'-CAGUACAAUCAUCGGGUUAUU-3' \\
& Antisense & 5'-P.UAACCCAUGAUUGUACUGUU-3' \\
P represents phosphate &
\end{tabular}

The double stranded RNA oligomer sequences for duplex 1 human CD98 were:

\section{Duplex}

Duplex 1 Sense Antisense 5'-P.AUCUUCACCAGACCAUUCUUU-3'

$P$ represents phosphate

Duplexes 2-4 were of unknown sequence. 
Control duplex used was siCONTROL Non-targeting siRNA \#2

\begin{tabular}{lll} 
Duplex & \multicolumn{1}{c}{ Sequence } \\
Control & Sense & 5'-UAAGGCUAUGAAGAGAUACUU-3' \\
duplex \#2 & Antisense & 5'-P.GUAUCUCUUCAUAGCCUUAUU-3' \\
P represents phosphate &
\end{tabular}

\subsubsection{Primers}

Primers were designed using Primer Express (Applied Biosystems) or using known/published primer sequences and purchased from MWG-Biotech unless otherwise specified.

The following primers were used in standard polymerase chain reactions (PCR) for the baculoviral expression system to verify the presence of the galectin-3 gene in the recombinant bacmid DNA (underlined sections of the primer sequence indicate restriction sites, $\mathrm{Ms}=$ mouse, $\mathrm{Hu}=$ human $)$ :

\section{Primer Sequence}

Ms Galectin-3 BamHI (F) 5'-CATGGATCCGATGGCAGACACGTTTTCGCT-3'

Ms Galectin-3 EcoRI (R) 5'-GCGGAATTCTTAGATCATGGCGTGGTTAGC-3'

M13 (F)

5'-GTTTTCCCAGTCACGAC-3'

M13 (R)

5'-CAGGAAACAGCTATGAC-3'

Hu Galectin-3 (F)

5'-ATGCGTTATCTGGGTCTGGA-3'

Hu Galectin-3 (R)

5'-TATGAAGCACTGGTGAGGTCTATG-3'

Ms Galectin-3 (F)

5'-CGCGGATCCATGGCAGACAGCTTTTCGCTT-3'

Ms Galectin-3 (R)

5'-GCGGAATTCTTAGATCATGGCGTGGTTAGC-3'

$(\mathrm{F}=$ Forward, $\mathrm{R}=$ Reverse $)$

The following primers were used for SYBR Green real-time RT-PCR:
Gene
Primer Sequence 


$$
\begin{array}{ll}
\text { Hu } \beta \text {-Actin (F) } & \text { 5'-CATCACCATTGGCAATGAGC-3' } \\
\text { Hu } \beta \text {-Actin (R) } & \text { 5'-CGATCCACACGGAGTACTTG-3' } \\
\text { Hu Mannose Receptor (F) } & \text { 5'-GCCAAATGACGAATTGTGGA-3' } \\
\text { Hu Mannose Receptor (R) } & \text { 5'-CACGAAGCCATTTGGTAAACG-3' } \\
\text { Ms } \beta \text {-Actin (F) } & \text { 5'-AGAGGGAAATCGTGCGTGAC-3' } \\
\text { Ms } \beta \text {-Actin (R) } & \text { 5'-CAATAGTGATGACCTGGCCGT-3' } \\
\text { Ms Mannose Receptor (F) } & \text { 5'-CATGAGGCTTCTCCTGCTTCT-3' } \\
\text { Ms Mannose Receptor (R) } & \text { 5'-TTGCCGTCTGAACTGAGATGG-3' } \\
\text { Ms NOS2 (F) } & \text { 5'-CAGCTGGGCTGTACAAACCTT-3' } \\
\text { Ms NOS2 (R) } & \text { 5'-CATTGGAAGTGAAGCGTTTCG-3' } \\
\text { Ms Arginase-1 (F) } & \text { 5'-TTGGGTGGATGCTCACACTG-3' } \\
\text { Ms Arginase-1 (R) } & \text { 5'-TTGCCCATGCAGATTCCC-3' } \\
\text { Ms YM-1 (F) } & \text { 5'-TCTCTACTCCTCAGAACCGTCAGA-3' } \\
\text { Ms YM-1 (R) } & \text { 5'-GATGTTTGTCTTTAGGAGGGCTTC-3', } \\
\text { Ms FIZZ-1 (F) } & \text { 5'-TACTTGCAACTGCCTGTGCTTACT-3' } \\
\text { Ms FIZZ-1 (R) } & \text { 5'-TATCAAAGCTGGGTTCTCCACCTC-3', } \\
\text { Ms IL4R } \alpha \text { (F) } & \text { 5'-ACCTGAGAACAGCGGAGGC-3' } \\
\text { Ms IL4R } \alpha(R) & \\
\text { (F = Forward, R = Reverse) } &
\end{array}
$$

The following primers and probes, purchased from Applied Biosystems, were used for TaqMan real-time RT-PCR reactions:

\section{Gene}

Ms Galectin-3 (F)

Ms Galectin-3 (R)

Ms Galectin-3 (P)

Ms $\alpha$-SMA (F)

Ms $\alpha$-SMA (R)

Ms $\alpha$-SMA (P)

Ms Pro-collagen I (F)

Ms Pro-collagen I (R)

\section{Primer/Probe Sequence}

5'-TTGAAGCTGACCACTTCAAGGTT-3'

5'-AGCTTCTTCATCCGATGGTTGT-3'

5'-6-FAM-CGGTCAACGATGCTCACCTACTGCATAMRA-3'

5'-TCAGCGCCTCCAGTTCCT-3'

5'-AAAAAAAACCACGAGTAACAAATCAA-3'

5'-6-FAM-TCCAAATCATTCCTGCCCA-TAMRA-3'

5'-TTCACCTACAGCACGCTTGTG-3'

5'-GATGACTGTCTTGCCCCAAGTT-3' 


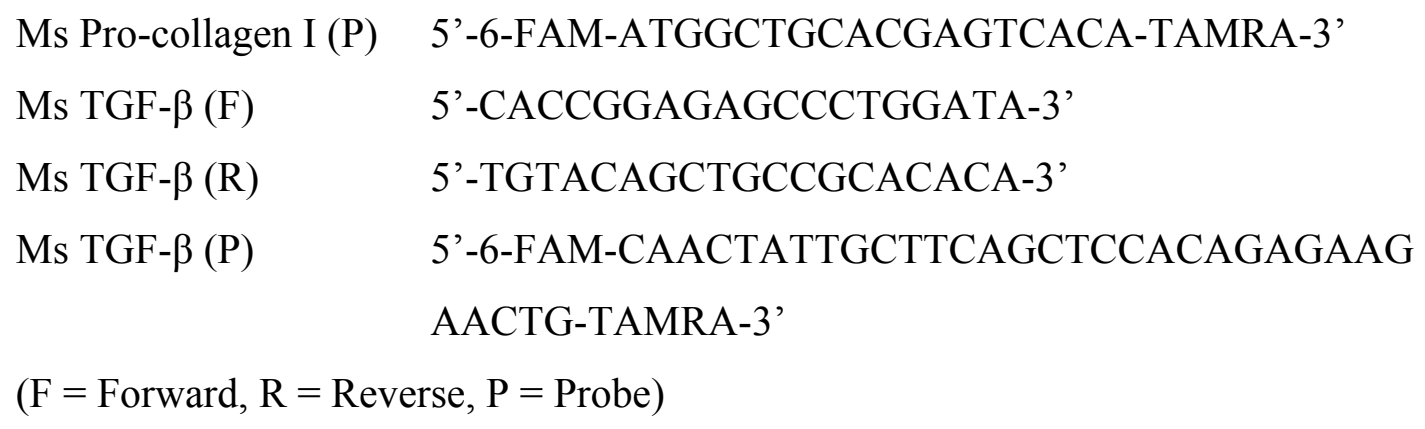

\subsubsection{Animals}

Generation of galectin-3 knockout (galectin- $3^{-/-}$) mice by gene targeting technology has been described (358). As controls, age- and sex-matched wild type littermate mice were used. All procedures were undertaken with approved licence from the Animal Scientific Procedure Division of the Home Office.

\subsection{Tissue culture}

HSC (Murine Hepatic Stellate cells), isolated from wild type and galectin- $3^{-/-}$ mice, were cultured in DMEM supplemented with 16\% heat inactivated FBS, $1 \%$ penicillin and streptomycin and 1\% L-glutamine. Cells were grown in a humidified atmosphere of $95 \% \mathrm{O}_{2} / 5 \% \mathrm{CO}_{2}$ at $37^{\circ} \mathrm{C}$. A detailed protocol for $\mathrm{HSC}$ isolation is given later in this chapter.

Sf9 (insect cell line, Invitrogen) cells cultured in Sf-900 II serum-free Insect Cell Culture Media (Invitrogen, Paisley, UK) plus 1\% amphotericin B (SigmaAldrich). Cells were grown at $27^{\circ} \mathrm{C}$.

THP-1 (human monocyte cell line, ATCC) were cultured in suspension in RPMI supplemented with 10\% heat inactivated FBS, 1\% penicillin and streptomycin and 1\% L-glutamine. Phorbol 12-myristate 13-acetate (PMA) (Sigma-Aldrich) treatment $(100 \mathrm{ng} / \mathrm{ml})$ was used to differentiate into macrophage-like cells, resulting in adherence to tissue culture plastic. Cells were grown in a humidified atmosphere of $95 \% \mathrm{O}_{2} / 5 \% \mathrm{CO}_{2}$ at $37^{\circ} \mathrm{C}$.

L929 (mouse fibroblast cell line) were cultured in DMEM-F12 supplemented with $10 \%$ heat inactivated FBS, $1 \%$ penicillin and streptomycin. Cells were grown 
in a humidified atmosphere of $95 \% \mathrm{O}_{2} / 5 \% \mathrm{CO}_{2}$ at $37^{\circ} \mathrm{C}$. Cells were cultured to confluency and culture the media was removed, filtered and frozen at $-80^{\circ} \mathrm{C}$ (this is L929 conditioned media).

BMDM (bone marrow derived macrophages) were isolated from wild type and galectin- $3^{-/-}$mouse femurs and tibias. Bone marrow was flushed with $10 \mathrm{ml}$ complete media (DMEM-F12 (Gibco) supplemented with 10\% L929 conditioned media as a source of granulocyte-macrophage colony-stimulating factor (GMCSF), $10 \% \mathrm{FBS}, 1 \%$ penicillin and streptomycin) through a 25 gauge syringe needle into tubes and the number of bone marrow cells counted using a haemocytometer. Cells were matured in complete DMEM-F12 at $10 \times 10^{6}$ cells per $10 \mathrm{~cm}$ plate for 7 days, with the addition of a further $10 \mathrm{ml}$ media half way through the maturation process. Maturation of BMDMs was examined by dual immunofluorescence staining for CD11b and F4/80 and analysed by flow cytometry. Macrophages were incubated with a 1:150 dilution of CD11b-PE antibody (clone M1/70) and a 1:25 dilution of F4/80-FITC antibody (clone CI:A3-1) for 30 minutes at room temperature in the dark. Samples were washed and a 1:10,000 dilution of the vital dye ToPro-3 (Invitrogen) was added to each tube just prior to flow cytometry analysis. Samples were analysed using a BD FacsCalibur flow cytometer.

Bone marrow derived neutrophils were isolated from wild type and galectin- $3^{-/-}$mouse femurs and tibias. Bone marrow was flushed out with $10 \mathrm{ml}$ sterile PBS, through a 25 gauge syringe needle. Cells were centrifuged at $400 \mathrm{xg}$ for 5 minutes, resuspended in $6 \mathrm{ml} 0.2 \% \mathrm{NaCl}$ for 45 seconds and osmolarity was immediately restored with the addition of $14 \mathrm{ml} 1.2 \% \mathrm{NaCl}$. The suspension was poured into a $50 \mathrm{ml}$ conical tube through a $70 \mu \mathrm{m}$ nylon cell strainer (Falcon), to remove clots and any bone remnants, pelletted and resuspended in $5 \mathrm{ml}$ PBS. $5 \mathrm{ml}$ of $62 \%$ Percoll (GE Healthcare) was layered at the bottom of a $15 \mathrm{ml}$ conical tube and the $5 \mathrm{ml}$ of cell suspension was gently layered on top. Tubes were centrifuged at $1000 \mathrm{xg}$ for 30 minutes at room temperature with acceleration and brake off. At the end of the gradient-centrifugation there was a sharp interface atop the $62 \%$ Percoll containing the immature cells and non-granulocyte lineages and a cloudier pellet containing the neutrophils. The cells at the interface were carefully discarded leaving $2-3 \mathrm{ml}$ of the $62 \%$ gradient to avoid disturbing the pellet. The pellet was 
transferred to another tube, washed $\mathrm{x} 2$, resuspended in $5-10 \mathrm{ml}$ of media and cells were counted using a haemocytometer. Cytospins were obtained from each isolation and GR-1 and CD11b positivity was assessed by fluorescent-activated cell sorting (FACS) analysis using anti-GR-1-PE (clone RB6-8C5) and anti-CD11b-APC (clone M1/70) antibodies. When needed, neutrophils were made apoptotic by culturing in suspension in RPMI supplemented with $10 \%$ heat inactivated FBS, $1 \%$ penicillin and streptomycin and $1 \%$ L-glutamine at $1 \times 10^{6}$ cells $/ \mathrm{ml}$ for up to 96 hours.

Peritoneal macrophages were isolated by flushing out wild type and galectin- $3^{-/-}$mouse peritoneal cavities. Mice were anaesthetised with a lethal dose of avertin (Tribromoethanol anaesthetic - 1.25\% 2,2,2-tribromoethanol plus 2.5\% 2methyl-2-butanol (components from Sigma-Aldrich)) or using $\mathrm{CO}_{2}$ exposure and any sign of pain response was checked by paw pinching. The peritoneal cavity was flushed out with 2 x $5 \mathrm{ml}$ volumes of sterile PBS and peritoneal lavages were placed on ice. Lavages were centrifuged, resuspended in DMEM containing $1 \%$ penicillin and streptomycin and adhered in 6-well plates at the required density.

Alveolar macrophages were isolated by flushing out wild type and galectin- $3^{-/-}$mouse lungs with $3 \times 1 \mathrm{ml}$ sterile PBS. Mice were anaesthetised with a lethal dose of avertin or using $\mathrm{CO}_{2}$ exposure and the lungs and trachea were exposed (with great care not to pierce any bit of the lungs). Elastic string was placed under the trachea and a small incision was made at the top of the trachea. A prepared plastic tube was inserted into the trachea and tied off using the elastic string. A $1 \mathrm{ml}$ syringe was prepared with $1 \mathrm{ml}$ of sterile PBS and inserted into the top of the tubing. The lungs were flushed with $3 \times 1 \mathrm{ml}$ of PBS (ensuring that both sides of the lungs inflate) and each lavage was recovered by drawing the liquid back into the syringe. Lavage was placed into a sterile $1.5 \mathrm{ml}$ eppendorf tube on ice. Lavages were centrifuged, resuspended in DMEM containing 1\% penicillin and streptomycin and adhered in 6-well plates at the required density. 


\subsection{Sodium dodececyl-sulphate polyacrylamide gel electrophoresis (SDS-PAGE) and Western Blotting}

\subsubsection{Cell Lysis}

Cells were washed once with PBS and lysed at $4{ }^{\circ} \mathrm{C}$ in an appropriate volume of lysis buffer. Lysates were shaken for 20 minutes at $4^{\circ} \mathrm{C}$ and cleared by centrifugation at $13 \mathrm{krpm}$ for 10 minutes at $4^{\circ} \mathrm{C}$, assessed for protein concentration using the BCA protein assay reagent (Pierce Biotechnology), and solubilised in $4 \mathrm{x}$ SDS-PAGE sample buffer at $95^{\circ} \mathrm{C}$ for 5 minutes. Samples were analysed immediately or stored at $-20^{\circ} \mathrm{C}$ for future analysis.

\subsubsection{SDS-PAGE and Western Blotting}

Buffers used are detailed in section 2.3.3. Samples were cooled or thawed from frozen and resolved on either $12 \%$ or $10 \%$ SDS-PAGE gel, under reducing and non-reducing conditions depending on the experiment, using a vertical electrophoresis tank Biorad Mini Protean II system. Samples were electrophoresed at 100-150 volts using electrophoresis buffer for 1-2 hours, adjacent to pre-stained molecular weight markers (Invitrogen). Proteins were transferred onto Hybond C nitrocellulose membranes (Amersham Pharmacia Biotech) in a methanol-based transfer buffer at 100 volts for 60 minutes, in a Mini Protean II blotting tank. Equal protein loading was confirmed by staining with 1\% Ponceau S (AMS Biotechnology) for 5 minutes to visualise protein bands. Non-specific binding sites were blocked by incubation with Tris-buffered saline (TBS)-Tween 20 (0.1\%) containing 5\% non-fat dried milk powder for 1 hour at room temperature. Membranes were probed with appropriate antibodies diluted in TBS-Tween 20 containing 5\% non-fat dried milk powder overnight at $4^{\circ} \mathrm{C}$. Membranes were then washed in TBS-Tween $20(3 \mathrm{x} 10$ minutes washes), before exposure to appropriate species-specific HRP-conjugated secondary antibodies (1:1000) for 1 hour at room temperature, diluted in TBS-Tween 20 containing 5\% non-fat dried milk powder. Membranes were then washed further in TBS-Tween 20 ( $3 \times 10$ minute washes), immunoreactive bands identified using enchanced chemiluminescence (ECL) (Amersham Pharmacia Biotech) according to the manufacturer's instructions and developed in a Konica SRX-101A film 
processor. Kodak MXB film (blue sensitive) was supplied by G.R.I Autoradiography (Genetic Research). For assessment of phosphorylated proteins, blocking and subsequent incubation steps were carried out using 3\% bovine serum albumin (BSA) in TBS-Tween 20.

\subsubsection{Buffers}

\section{Lysis buffer:}

25mM 4-(2-hydroxyethyl)-1-piperazineethanesulfonic acid (HEPES) pH 7.4, 0.3M $\mathrm{NaCl}, 1.5 \mathrm{mM} \mathrm{MgCl} 2,0.2 \mathrm{mM}$ ethylenediaminetetraacetic acid (EDTA), 0.5\% Triton X-100 (v/v), 1mM sodium orthovanadate, $0.5 \mathrm{mM}$ dithiothreitol (DTT). One Complete $^{\mathrm{TM}}$ protease inhibitor tablet (Roche) was added per $50 \mathrm{ml}$ of lysis buffer. Non-reducing buffer contains no DTT.

\section{4x SDS-PAGE sample buffer:}

50mM Tris-HCl, 10\% glycerol (v/v), 2\% SDS (v/v), 0.1\% bromophenol blue (v/v), $10 \% \beta$-mercaptoethanol $(\mathrm{v} / \mathrm{v}), \mathrm{pH}$ 6.8. Non-reducing buffer contains no $\beta$ mercaptoethanol.

\section{SDS-polyacrylamide gels:}

Separating Gel:

$0.375 \mathrm{M}$ Tris base ( $\mathrm{pH} 8.8), 0.1 \%$ SDS (v/v), 10-12\% acrylamide (v/v), 0.1\% ammonium persulphate (v/v), 0.02\% Tetramethylethylenediamine (TEMED) (v/v).

Stacking Gel:

$0.13 \mathrm{M}$ Tris base ( $\mathrm{pH} 6.8), 0.1 \%$ SDS (v/v), 4.5\% acrylamide (v/v), $0.1 \%$ ammonium persulphate $(\mathrm{v} / \mathrm{v}), 0.02 \%$ TEMED $(\mathrm{v} / \mathrm{v})$.

\section{1x Gel electrophoresis running buffer:}

$50 \mathrm{mM}$ Tris base, $250 \mathrm{mM}$ glycine, $0.1 \% \operatorname{SDS}(\mathrm{v} / \mathrm{v})$.

\section{Transfer buffer:}

210mM glycine, $24.7 \mathrm{mM}$ Tris base, $20 \%$ methanol (v/v) (Fisher Scientific) 
$\underline{\text { TBS-Tween } 20 \text { wash buffer: }}$

$150 \mathrm{mM} \mathrm{NaCl}, 20 \mathrm{mM}$ Tris $\mathrm{HCl}(\mathrm{pH} 7.4), 0.1 \%$ Tween 20 (v/v)

$\begin{array}{llll}\text { Primary antibody } & \text { Dilution } & \text { Secondary Antibody } & \text { Dilution } \\ \text { Actin } & 1: 5000 & \text { Anti-Rabbit HRP } & 1: 1000 \\ \text { Galectin-3 (A3A12) } & 1: 2000 & \text { Anti-Mouse HRP } & 1: 1000 \\ \text { a-SMA (1A4) } & 1: 15,000 & \text { Anti-Mouse HRP } & 1: 1000 \\ \text { pSmad-2/3 } & 1: 1000 & \text { Anti-Goat HRP } & 1: 1000 \\ \text { pSmad-3 } & 1: 1000 & \text { Anti-Rabbit HRP } & 1: 1000 \\ \text { pPKC } & 1: 1000 & \text { Anti-Rabbit HRP } & 1: 1000 \\ \text { pERK 1-2 (MAPK-YT) } & 1: 1000 & \text { Anti-Mouse HRP } & 1: 1000 \\ \text { pAKT/PKB (14-6) } & 1: 500 & \text { Anti-Rabbit HRP } & 1: 1000 \\ \text { pJNK 1-2 } & 1: 500 & \text { Anti-Rabbit HRP } & 1: 1000 \\ \text { YM-1 } & 1: 1000 & \text { Anti-Rabbit HRP } & 1: 1000 \\ \text { Human CD98 } & 1: 1000 & \text { Anti-Goat HRP } & 1: 1000 \\ \text { Human Mannose R (15-2) } & 1: 1000 & \text { Anti-Mouse HRP } & 1: 1000 \\ \text { pSTAT-6 } & 1: 500 & \text { Anti-Rabbit HRP } & 1: 1000 \\ \text { PKB } & 1: 1000 & \text { Anti-Rabbit HRP } & 1: 1000 \\ \text { Arginase-1 } & 1: 500 & \text { Anti-Rabbit HRP } & 1: 1000 \\ \text { Mouse CD98 } & 1: 1000 & \text { Anti-Goat HRP } & 1: 1000\end{array}$

Table 2.1 - Immunoblotting antibody dilutions.

\subsection{Protein Purification}

\subsubsection{Bacterial expression system}

Escherichia coli (E. coli) BL-21 star (DE3) bacteria (Stratagene) transformed with pcDNA 3.1 vector containing a histidine (His)-tagged human galectin-3 insert, was used to extract His-tagged human galectin-3 protein. 
Figure 2.1 - Map of pcDNA 3.1.

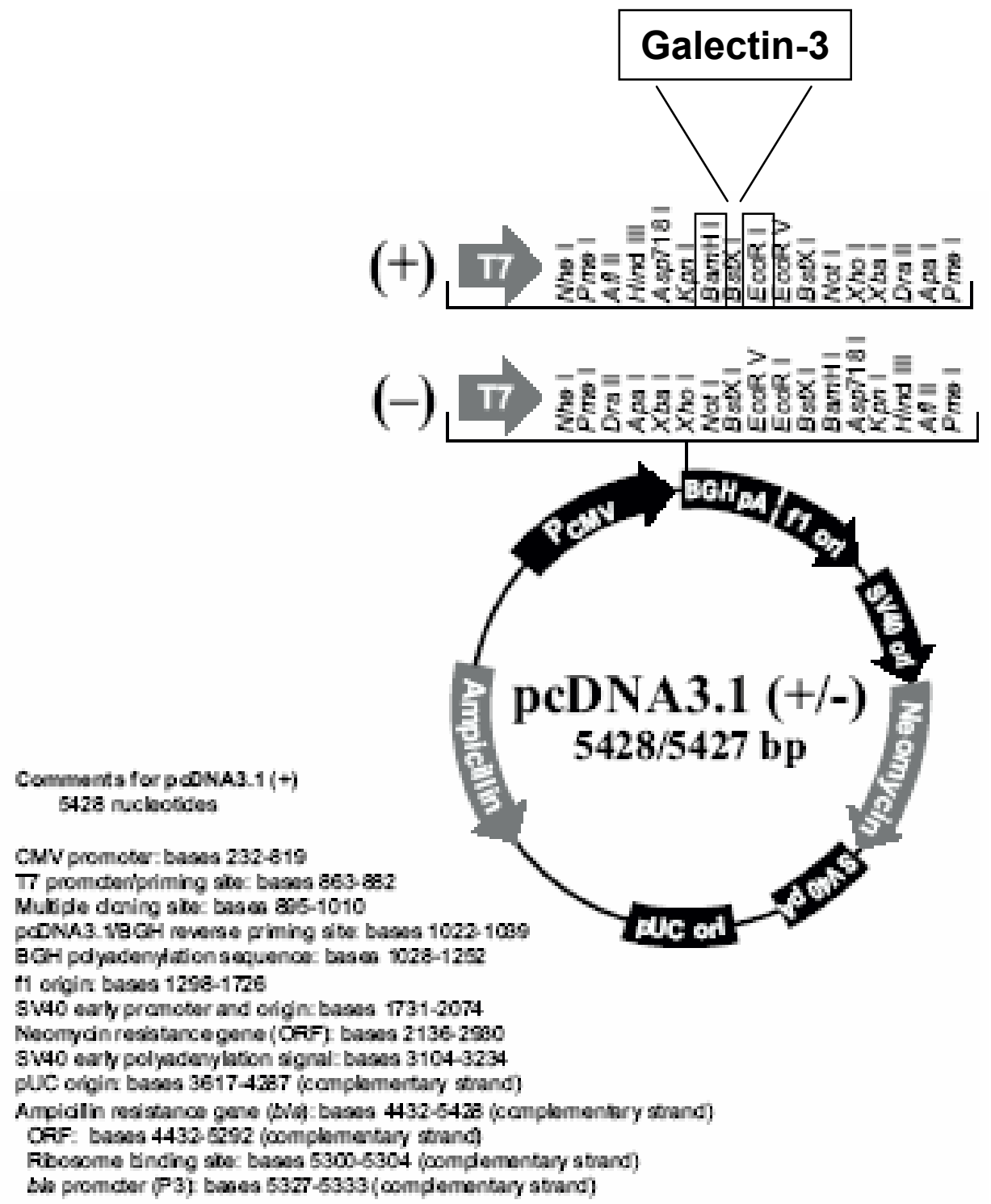

Figure 2.1

His-tagged galectin-3 was cloned into a pcDNA 3.1 vector between BamH I and EcoR I restriction sites by a previous member of this laboratory. This recombinant vector was then used to transform BL-21 star bacteria. 
Transformation of BL-21 star bacteria was performed as follows. $0.5 \mu \mathrm{g}$ of pcDNA 3.1 vector, with a His-tagged galectin-3 insert (cloned by a previous member of this laboratory) (figure 2.1) was added to $100 \mu 1$ of bacteria, mixed gently and placed on ice for 30 minutes. Bacteria were heat shocked for 50 seconds at $42^{\circ} \mathrm{C}$ and immediately transferred to ice for 2 minutes. $900 \mu$ of S.O.C (S.O.C is Super Optimal Broth (S.O.B) with glucose; $2 \%$ tryptone, $0.5 \%$ yeast extract, $8.6 \mathrm{mM} \mathrm{NaCl}$, $2.5 \mathrm{mM} \mathrm{KCl}, 20 \mathrm{mM} \mathrm{MgSO}_{4}$ and $20 \mathrm{mM}$ glucose) was added and tubes were incubated for 45 minutes at $37^{\circ} \mathrm{C}$ shaking at $700 \mathrm{rpm}$. Bacteria were pelletted for 5 minutes at $5 \mathrm{krpm}$ and resuspended in $200 \mu$ S.O.C. Neat, 1:10, 1:50 and 1:100 dilutions were plated onto Luria-Bertani (LB) (Sigma-Aldrich) agar plates containing $50 \mu \mathrm{g} / \mathrm{ml}$ ampicillin (Sigma-Aldrich). A negative control plate was prepared by plating out untransformed BL-21 bacteria onto LB agar plates containing $50 \mu \mathrm{g} / \mathrm{ml}$ ampicillin. The next day colonies were selected and inoculated into $10 \mathrm{ml}$ of LB broth (made up using $1 \mathrm{LB}$ broth base tablet (Sigma-Aldrich) per $50 \mathrm{ml}$ distilled $\mathrm{H}_{2} \mathrm{O}$ ) containing $50 \mu \mathrm{g} / \mathrm{ml}$ ampicillin and allowed to grow overnight. The following day, $2.5 \mathrm{ml}$ of the culture was inoculated into $500 \mathrm{ml}$ flasks in LB broth containing $50 \mu \mathrm{g} / \mathrm{ml}$ ampicillin. Bacteria were allowed to grow to an optical density (OD) 600 of 0.7 to 0.8 , induced with $1 \mathrm{mM}$ isopropyl $\beta$-D-thiogalactoside (IPTG) (Sigma-Aldrich) and then grown overnight at $37^{\circ} \mathrm{C}$ with shaking. Cultures were centrifuged at 8,000rpm in a Beckman J2-MC ultracentrifuge for 20 minutes. Pellets were resuspended in $5 \mathrm{ml}$ of BugBuster Protein Extraction Reagent (Novagen) per gram of wet cell paste followed by the addition of $1 \mu \mathrm{l} / \mathrm{ml}$ Benzonase (Novagen). This was separated into $50 \mathrm{ml}$ conical tubes, placed on a rotary shaker for 20 minutes and centrifuged at $11,500 \mathrm{rpm}$ in a Heraus Multifuge $3 \mathrm{~S}-\mathrm{R}$ for 20 minutes. Galectin-3 was purified from the cleared lysate using Ni-NTA His·Bind ${ }^{\circledR}$ Resin. $1 \mathrm{ml}$ of NiNTA His·Bind ${ }^{\circledR}$ Resin (Novagen), per purification, was washed with $4 \mathrm{ml} 1 \mathrm{X}$ NiNTA Bind Buffer $(300 \mathrm{mM} \mathrm{NaCl}, 50 \mathrm{mM}$ sodium phosphate buffer, $10 \mathrm{mM}$ imidazole, $\mathrm{pH}$ 8.0), the beads were allowed to settle by gravity or centrifuged at $1000 \mathrm{rpm}$ for 5 minutes, and the top bind buffer layer was removed. $4 \mathrm{ml}$ of cleared lysate was loaded onto the prepared Ni-NTA His·Bind slurry and mixed gently by shaking at $4{ }^{\circ} \mathrm{C}$ for at least 60 minutes. This was then loaded onto a disposable polypropylene column (1$5 \mathrm{ml}$ bed volume) (Pierce Biotechnology), flow through collected (aliquot taken for 
SDS-PAGE analysis) and 3 washes were carried out using Ni-NTA Wash Buffer (300mM NaCl, 50mM sodium phosphate buffer, 20mM imidazole, $\mathrm{pH} 8.0$ ), (aliquots of washes taken for SDS-PAGE analysis). 4x 500 $\mu 1$ elutions were performed with Ni-NTA Elution Buffer (300mM NaCl, 250mM imidazole, 50mM sodium phosphate buffer, $\mathrm{pH} 8.0$ ) and elutions were collected in $4 \mathrm{x} 1.5 \mathrm{ml}$ eppendorf tubes. Fractions were analysed using SDS-PAGE and elutions dialysed against $1 \mathrm{~L}$ cold $1 \mathrm{X}$ PBS overnight (dialysis tubing size $-14.3 \mathrm{~mm}$, molecular weight cut off 12-14KDa). Protein determination using Pierce BCA protein assay reagent was performed and Western blots used to verify the presence of galectin-3.

\subsubsection{Baculovirus Expression System}

The Invitrogen Baculovirus Expression System was used to produce recombinant baculovirus containing the gene of interest, in this case human and mouse galectin-3. Detailed protocols are given in the Bac-to-Bac Baculovirus Expression System manual (Invitrogen) and in Chapter 3. Briefly, galectin-3 DNA was cloned into a pFastBac donor plasmid (Invitrogen) which comprises a baculoviral specific promoter. The pFastBac construct was then transformed into the E.coli host strain DH10Bac (Invitrogen) which contains a baculovirus shuttle vector (bacmid) and a helper plasmid. Recombinant bacmid was produced upon transposition of the pFastBac construct which was purified using the S.N.A.P midiprep kit (Invitrogen). Bacmid was transfected into Sf9 insect cells and amplified using the method given in the manual. When sufficient amount of virus was produced, $3 \times \mathrm{T} 162 \mathrm{~cm}^{2}$ flasks containing $3 \times 10^{7} \mathrm{Sf} 9$ cells in $30 \mathrm{ml}$ growth media were infected and protein purified using the Ni-NTA His bind ${ }^{\circledR}$ resin as detailed above.

\subsection{Immunohistochemistry (IHC)}

$10 \%$ Formalin (Sigma-Aldrich) fixed liver or kidney or methyl carnoys $(60 \%$ methanol, 30\% chloroform, 10\% glacial acetic acid)-fixed kidney was paraffin embedded by the histology department at the University of Edinburgh who also prepared $3 \mu \mathrm{m}$ thick sections of tissue onto superfrost slides (Fisher Scientific) using a microtome. $10 \mu \mathrm{m}$ sections were prepared for PSR staining. Archival human liver 
samples were obtained from the University of Edinburgh Department of Pathology files. Rat liver sections were obtained from Professor John Iredale (University of Edinburgh). Samples had been fixed in formalin and embedded in paraffin wax.

Sections were dewaxed in xylene (Fisher Scientific) and rehydrated through graded ethanol solution from $100 \%$ to $50 \%$ ethanol. Antigen retrieval was performed by microwaving the formalin-fixed tissue sections for 15 minutes at full power in antigen unmasking solution which is based on a citric acid formula (Vector). F4/80 IHC did not have an antigen retrieval step. Endogenous peroxidase was blocked by incubating the sections in 3\% hydrogen peroxide $\left(\mathrm{H}_{2} \mathrm{O}_{2}\right)$ (VWR (BDH)) for 15 minutes. After non-specific binding was blocked using the avidin/biotin blocking kit (Vector) and either Dako protein block or blocking serum from the Vector Quick Kit, the sections were incubated with the relevant antibody either overnight at $4{ }^{\circ} \mathrm{C}$ or at room temperature for the required amount of time depending on the antibody used.

Antibodies used on formalin-fixed tissue included 1:200 anti-mouse Mac-2FITC monoclonal antibody (anti-mouse galectin-3) (clone M3/38), 1:100 anti-human galectin-3 (clone 9C4), 1:100 anti-rat galectin-3 and 1:15,000 anti- $\alpha$-SMA-FITC (clone 1A4). 1:250 anti-mouse F4/80 (clone CI: A3-1) was used on methyl carnoysfixed tissue sections and did not get antigen retrieval. After incubation with FITClabelled primary antibodies against mouse galectin- 3 or $\alpha$-SMA, sections were washed $\mathrm{x} 2$ with TBS and incubated with rabbit anti-FITC secondary antibody (1:4000) for 30 minutes at room temperature. Sections were washed $\mathrm{x} 2$ with TBS then incubated with biotin-conjugated anti-rabbit antibody (1:200) for 30 minutes at room temperature, washed $\mathrm{x} 2$ with TBS, then incubated with an avidin:biotinylated enzyme complex (ABC) (Vector) for a further 30 minutes at room temperature. The DakoCytomation EnVision+ System-HRP was used for human galectin-3 IHC, the Vector Quick Kit was used for mouse F4/80 IHC and biotinylated goat anti-rabbit secondary antibody (1:300) followed by the avidin:biotinylated enzyme complex was used for anti-rat galectin-3 IHC. Diaminobenzidine (DAB) (DakoCytomation) solution was subsequently added for 5 minutes. Sections were counterstained for 5 minutes with haematoxylin (ThermoShandon) and 5 minutes with Scott's tap water $\left(83 \mathrm{mM} \mathrm{MgSO}_{4}, 7.1 \mathrm{mM} \mathrm{NaHCO}\right.$ in tap water), dehydrated in graded ethanol from $50 \%$ to $100 \%$ and cleared in xylene. Slides were mounted using Pertex mounting 
solution (CellPath Hemel Hempstead, UK). In each experiment, negative controls of secondary antibody only and relevant isotype controls (DakoCytomation) were performed.

Collagen fibres were stained with picrosirius red (PSR) as follows. Sections were dewaxed and rehydrated as above and washed $\mathrm{x} 2$ in running tap water for 5 minutes. Slides were placed in PSR solution -1 part 1\% Sirius Red (1g Sirius Red, $1 \mathrm{~g}$ Fast Green, $100 \mathrm{ml}$ distilled $\left.\mathrm{H}_{2} \mathrm{O}\left(\mathrm{dH}_{2} \mathrm{O}\right)\right)$ with 9 parts saturated aqueous picric acid) - for 2 hours, washed in running tap water for 1 minute, dehydrated, cleared and mounted as above.

Haematoxylin and eosin (H\&E) stain was performed as follows. Sections were dewaxed and rehydrated as above and washed $\mathrm{x} 2$ in running tap water for 5 minutes. Tissue sections were incubated in haematoxylin for 5 minutes followed by $1 \%$ acid alcohol solution ( $70 \%$ industrial methylated spirits, $1 \% \mathrm{HCl})$ for 5 minutes. Sections are incubated in Scott's tap water for 1 minute and then Eosin for 1 minute. Sections were washed in running tap water for 1 minute, dehydrated, cleared and mounted as above.

Images were acquired using a Leica DM LB light microscope, Leica DC 300 digital camera and Leica IM50 software.

\subsection{Quantitation of IHC using Openlab}

Images of PSR and $\alpha$-SMA staining in liver and kidney sections were quantified using the Openlab software. Set-up of this protocol was carried out with the help of Dr Gareth Clegg (University of Edinburgh) who has substantial experience with the Openlab software. This method uses the software's density slicing module to locate objects by their colour or their intensity. The resulting binary layer can be measured using Openlab's measurement modules.

Morphometric measurements were made on 10 random fields from $10 \mu \mathrm{m}$ or $3 \mu \mathrm{m}$ sections stained with PSR or $\alpha$-SMA respectively using Openlab software (Improvision, UK). Ten random fields from each tissue section from each mouse were analysed. Each captured field was analysed by separation into red, green and blue (RGB) filters. The area of staining was selected by altering the values of the red, green and blue channels in the density slicing module until all areas of positive 
staining were highlighted. The same values were used for each image. Openlab then creates a binary layer highlighting all the areas of positive staining. The measurement module of the software was used to measure the area highlighted by the density slice module. The area of positive staining was mathematically divided by the RGB area and multiplied by $100 \%$. This represents the percentage area staining positively for collagen or $\alpha$-SMA, providing a quantitative value on a continuous scale.

\section{$2.7 \quad$ HSC Isolation}

Wild type and galectin- $3^{-/-}$mouse HSC were extracted from mouse liver by in situ perfusion with pronase (Roche) and collagenase B (Roche) followed by density gradient centrifugation using Optiprep (Axis Shield). Due to the nature of this protocol and the requirement for two people to perform this procedure, I performed the HSC isolations with the help of either Dr Neil Henderson or Dr Alison Mackinnon.

Enzymes were prepared, under sterile conditions, to the following dilutions in HBSS $(+)$ containing calcium $\left(\mathrm{Ca}^{2+}\right)$ and magnesium $\left(\mathrm{Mg}^{2+}\right)$, fully dissolved and sterile filtered (the following volumes are for a maximum of 8 mice); $30 \mathrm{ml}$ of $2 \mathrm{mg} / \mathrm{ml}$ collagenase B, $30 \mathrm{ml}$ of $12 \mathrm{mg} / \mathrm{ml}$ pronase, $80 \mathrm{ml}$ of $1 \mathrm{mg} / \mathrm{ml}$ DNase I (Roche). $12 \mathrm{ml}$ of enyme mix was prepared by mixing $6 \mathrm{ml}$ of filtered collagenase solution with $6 \mathrm{ml}$ of filtered pronase solution.

Liver perfusions were carried out using autoclaved instruments soaked in ethanol before use and each mouse was sprayed with ethanol before surgery to avoid bacterial contamination. Mice were anaesthetised with a lethal dose of avertin, the peritoneal cavity was opened and the portal vein cannulated with a 30 gauge needle (on a syringe containing $1 \mathrm{ml}$ enzyme mix), the Inferior Vena Cava was pierced with a needle and, immediately but slowly, the liver was flushed with the enzyme mix causing it to blanch. The liver was removed and placed into a sterile tube containing a small volume of HBSS, enough to cover the livers.

Once back in the tissue culture laboratory, the rest of the protocol was carried out under sterile conditions using a sterile laminar flow cabinet. The four livers in HBSS $(+)$ were divided into $3 \times 25 \mathrm{ml}$ universals for each group (3 for wild type and 3 
for galectin- $3^{-/-}$). The rest of the collagenase and pronase solution were combined and made up to $48 \mathrm{ml}$ with $\operatorname{HBSS}(+)$ and $8 \mathrm{ml}$ of this mix was added to each universal. Livers were chopped up using autoclaved and ethanol sterilised scissors, the universals were sealed, wrapped in parafilm and shaken at $37^{\circ} \mathrm{C}$ for 20 minutes at 290rpm. $5 \mathrm{ml}$ of filtered DNase solution was added to each digest, mixed by pipetting and the extracts from the four livers were combined by pouring through autoclaved 125U nybolt mesh (John Staniar and co.) carefully taped onto plastic sterilin pots ( 1 for wild type and 1 for galectin- $3^{-/}$). The digest was carefully grinded through the filter using the end of a sterile $10 \mathrm{ml}$ stripette and $\mathrm{HBSS}(+)$ was used to wash as much of the pulp as possible through the filter, keeping the total volume collected in the sterilin $<100 \mathrm{ml}$. The resulting digest was split into $50 \mathrm{ml}$ conical tubes ( 2 for wild type and 2 for galectin- $3^{-/-}$) and centrifuged at room temperature for 7 minutes at 400xg. Supernatants were removed, without disturbing the pellet, and each pellet resuspended in $3 \mathrm{ml}$ filtered DNase solution, made up to $30 \mathrm{ml}$ with $\operatorname{HBSS}(+)$ and centrifuged at room temperature for a further 7 minutes at $400 \mathrm{xg}$. As much of the supernatant as possible was poured off, without disturbing the pellet, and each pellet was resuspended in $3 \mathrm{ml}$ DNase. Volumes were measured using a $10 \mathrm{ml}$ stripette and made up to $8.9 \mathrm{ml}$ total volume with $\mathrm{HBSS}(+)$. $5.9 \mathrm{ml}$ neat Optiprep was added to each cell suspension and mixed. In a $50 \mathrm{ml}$ conical tube, $21.6 \mathrm{ml}$ neat Optiprep was mixed with $14.4 \mathrm{ml} \mathrm{HBSS}(+)$ and divided into $8 \mathrm{x} 15 \mathrm{ml}$ conical tubes, $4 \mathrm{ml}$ per tube (this is the bottom layer of the gradient). $7.4 \mathrm{ml}$ of each cell suspension/Optiprep mix was very carefully and slowly layered on top of the gradient of each of the $15 \mathrm{ml}$ conical tubes ( 4 for wild type and 4 for galectin- $3^{-/}$). $500 \mu 1$ of $\operatorname{HBSS}(+)$ was layered on top and centrifuged at $4{ }^{\circ} \mathrm{C}$ for 20 minutes at $1400 \mathrm{xg}$ ensuring that the acceleration and brake is off. After gradient centrifugation the top HSC layers were transferred to a $50 \mathrm{ml}$ conical tube, using a sterile Pasteur pipette, containing $3 \mathrm{ml}$ DNase, combining wild type together and galectin ${ }^{-/-}$together. $30 \mathrm{ml} \mathrm{HBSS}(+)$ was added to each tube and centrifuged at room temperature for 7 minutes at $400 \mathrm{xg}$. The supernatants were carefully discarded, each pellet resuspended in $10 \mathrm{ml}$ complete DMEM and counted. Extracted HSC were cultured on plastic until they were activated to a myofibroblast phenotype after 7-10 days and used for experiments after activation in primary culture or before the fourth passage. 
Cells were cultured in DMEM supplemented with 16\% heat inactivated FBS, $1 \%$ penicillin and streptomycin and 1\% L-glutamine.

\subsection{Immunofluorescence (IMF)}

Cells were seeded onto coverslips (Fisher Scientific) or into Lab-Tek chamber slides (Nunc) at the required density and treated as necessary (see Results Chapters for details). Galectin-3 and $\alpha$-SMA IMF was performed on HSCs and YM1 IMF was performed on alveolar macrophages. Cells were fixed with 3\% paraformaldehyde (PFA) and permeabilised with $0.05 \%$ Triton for galectin-3 and YM-1 IMF or both $0.1 \%$ and $0.05 \%$ Triton for $\alpha$-SMA IMF. Non-specific binding was blocked with fish skin gelatin (FSG) for 1 hour at room temperature. Primary antibodies were added at the following dilutions in FSG; 1:20 for galectin-3 (clone A3A12), 1:100 for $\alpha$-SMA (clone 1A4) and 1:1000 for YM-1 for 1 hour at room temperature or overnight at $4^{\circ} \mathrm{C}$. Cells were washed with $3 \times 5$ min PBS washes. Alexa-fluor-conjugated secondary antibodies were added at 1:1000 for 1 hour at room temperature followed by $3 \times 0.05 \%$ Triton washes and $1 \times$ PBS wash. To stain the nuclei $0.1 \mu \mathrm{g} / \mathrm{ml} \mathrm{4',6-diamidino-2-phenylindole} \mathrm{(DAPI)} \mathrm{was} \mathrm{added} \mathrm{in} \mathrm{the} \mathrm{third}$ triton wash. Slides were immediately mounted in Mowiol 4-88 (Calbiochem) analysed using a Zeiss Axiovert S100 microscope and subsequently processed using Openlab software and Adobe Photoshop. Mowiol was prepared by mixing $2.4 \mathrm{~g}$ Mowiol 4-88 and $6 \mathrm{~g}$ glycerol in $6 \mathrm{ml}$ water for several hours at room temperature. $12 \mathrm{ml}$ of $0.2 \mathrm{M}$ Tris $(\mathrm{pH} 8.5)$ was added and heated at $50^{\circ} \mathrm{C}$ for 10 minutes. After the Mowiol dissolved, the solution was clarified by centrifugation at $5000 \mathrm{xg}$ for 15 minutes.

\subsection{SiRNA transfections}

HSCs were transfected with mouse galectin-3 siRNA and THP-1 cells were transfected with human galectin-3 or CD98 siRNA. HSCs were seeded at $2 \times 10^{5}$ cells/well in 6-well plates and transfected with siRNA after 7 days in culture (final concentration $125-250 \mathrm{nM}$ ) using Oligofectamine Reagent (Invitrogen) according to manufacturer's instructions. 
Oligonucleotide-Oligofectamine complexes were prepared as follows (volumes given represent one reaction for one well of a 6-well plate). One tube was prepared containing the required concentration of siRNA plus $175 \mu 1$ serum-free media. A second tube was prepared containing $4 \mu 1$ of Oligofectamine plus $15 \mu 1$ serum-free media. After a 5-10 minute incubation of Oligofectamine with the media, the diluted siRNA and the diluted Oligofectamine were combined (total volume $\sim 200 \mu 1$ ), mixed gently and incubated for 20 minutes at room temperature. This allows the siRNA-Oligofectamine complexes to form. While the complexes were forming, the growth media was removed from the cells, washed once with serumfree medium and $800 \mu 1$ of serum-free medium was added to the well. The $200 \mu 1$ of siRNA-Oligofectamine complex was gently mixed and added to the $800 \mu 1$ medium in the well. The cells were incubated at $37^{\circ} \mathrm{C}$ in a $\mathrm{CO}_{2}$ incubator for 4 hours. After 4 hours the transfection mixture was removed and replaced with complete growth media. HSCs were lysed after 96 hours or incubated with relevant treatments (see Results Chapters for details).

THP-1 cells were differentiated with 100ng/ml PMA for 24 hours, washed and transfected with $100 \mathrm{nM}$ galectin-3 siRNA duplexes or 120nM CD98 siRNA duplexes using Oligofectamine as described above. Cells were incubated for 48 hours in complete media prior to the addition of cytokines. siRNA was purchased from Dharmacon Research (sequences given above). Control duplex was siCONTROL nontargetting siRNA no. 2.

\subsection{Real-Time RT-PCR}

\subsubsection{RNA extractions}

RNA was prepared from cell lines and whole livers and kidneys using QIAshredder columns and the RNeasy Mini Kit (Qiagen) according to the manufacturer's instructions. RNA concentrations were determined using a WPA UV 1101 Biotech photometer and RNA was stored at $-80^{\circ} \mathrm{C}$ until further use. 


\subsubsection{RNA extraction from whole liver and kidney}

Livers or kidneys were removed from mice, a piece of liver or one quarter of each kidney was snap frozen in liquid nitrogen and stored at $-80^{\circ} \mathrm{C}$ until further use. $5 \mathrm{~mm}$ stainless steel, autoclaved beads (Qiagen) were placed into sterile $2 \mathrm{ml}$ lock eppendorf tubes, one bead per tube, under sterile conditions and remained sterile until further use.

Under sterile conditions, $600 \mu 1$ RLT buffer with $6 \mu 1 \beta$-mercaptoethanol ( $\beta$ $\mathrm{Me}$ ) was added to each $2 \mathrm{ml}$ tube plus bead. Livers or kidneys were transferred to dry ice and, under sterile conditions and using a separate sterile scalpel per piece of tissue, a small segment (less than 30mg) was removed and immediately placed into the sterile $2 \mathrm{ml}$ tube plus bead containing RLT/ $\beta$-Me mix. The remaining tissue was returned to the $-80^{\circ} \mathrm{C}$ freezer as quickly as possible. The tissue was homogenised by shaking the tubes in a TissueLyser (Qiagen) at $20 \mathrm{~Hz}$ for $1-2$ minutes (x2). RNA was extracted by pipetting the resulting lysate into QIAshredder columns and purified using the RNeasy Mini Kit according to the manufacturer's instructions.

\subsubsection{DNase treatment of RNA}

In autoclaved $1.5 \mathrm{ml}$ eppendorf tubes, $2 \mu \mathrm{RQ} 1 \mathrm{RNase}-$ Free DNase (Promega) and $2 \mu 110 \mathrm{x}$ reaction buffer (Promega) was added to $2 \mu \mathrm{g}$ RNA, made up to a final volume of $20 \mu 1$ with nuclease-free $\mathrm{H}_{2} \mathrm{O}\left(\mathrm{NF}_{2} \mathrm{O}\right)$ and incubated for 30 minutes at $37^{\circ} \mathrm{C}$. Immediately, $2 \mu 1 \mathrm{RQ} 1 \mathrm{DNase}$ stop solution (Promega) was added to each tube for 10 minutes at $65^{\circ} \mathrm{C}$ to terminate the reaction. 'Clean' RNA was stored at $-80^{\circ} \mathrm{C}$ until further use.

\subsubsection{PCR to check for DNA contamination}

After DNase treatment, clean RNA was checked for DNA contamination by PCR using $\beta$-actin primers (see Materials). Appropriate positive (species matched complementary DNA (cDNA) in place of RNA) and negative $\left({ }_{\mathrm{NF}} \mathrm{H}_{2} \mathrm{O}\right.$ in place of RNA) controls were used. The following volumes represent one PCR reaction: 


\section{Reaction mix:}

10x Taq Buffer $\left(+\mathrm{MgCl}_{2}\right)$ (Promega) $2 \mu \mathrm{l}$

dNTP (Applied Biosystems) $\quad 0.4 \mu \mathrm{l}$

Taq DNA polymerase B (Promega) $\quad 0.2 \mu 1$

Forward Primer

Reverse Primer

${ }_{\mathrm{NF}} \mathrm{H}_{2} \mathrm{O}$

RNA

Total volume

\section{Amplification conditions:}

$0.8 \mu 1$

$0.8 \mu 1$

$13.8 \mu 1$

$2 \mu 1$

$20 \mu 1$ $94^{\circ} \mathrm{C} \quad 2 \mathrm{~min}$

$94^{\circ} \mathrm{C} \quad 30 \mathrm{sec}$

$56^{\circ} \mathrm{C} \quad 30 \mathrm{sec}$

$72^{\circ} \mathrm{C} \quad 30 \mathrm{sec}$

$40 \mathrm{x}$

The PCR reaction was carried out in a PTC-100 programmable thermal controller (MJ Research Inc). 10 $\mu$ l of each PCR reaction was resolved on $2 \%$ agarose gels (Seakem) made with 1x TAE (Tris-Acetate-EDTA; 40mM Tris Base, $0.1 \%$ Acetic Acid, $0.2 \%$ EDTA, $\mathrm{pH} 8$ ) containing $0.3 \mu \mathrm{g} / \mathrm{ml}$ ethidium bromide (VWR $(\mathrm{BDH}))$ to enable UV visualisation. $100 \mathrm{bp}$ and $1 \mathrm{~kb}$ DNA ladder were purchased from Promega. Bands were visualised with a White/Ultraviolet TMV-20 Transilluminator (UVP Ultraviolet Products) and pictures taken using UVP Grab-IT version 2.5 (UVP Ultraviolet Products).

\subsubsection{Real-Time Reverse Transcription-PCR (Real-Time RT-PCR)}

DNase-treated RNA extracted from cell lines or whole kidney was reversed transcribed into cDNA using TaqMan $^{\circledR}$ reverse transcription reagents (Applied Biosystems). The following volumes represent one reverse transcription (RT) reaction:

Reaction mix:

10x TaqMan RT Buffer

$25 \mu \mathrm{M} \mathrm{MgCl}_{2}$

dNTP

Random Hexamers

RNase Inhibitor

Multiscribe reverse transcriptase

RNA

Total volume
Conditions:

$4 \mu 1$

$8.8 \mu \mathrm{l} \quad 25^{\circ} \mathrm{C} \quad 10 \mathrm{~min}$

$8 \mu \mathrm{l} \quad 48^{\circ} \mathrm{C} \quad 40 \mathrm{~min}$

$2 \mu 1 \quad 95^{\circ} \mathrm{C} \quad 5 \mathrm{~min}$

$0.8 \mu 1 \quad 4^{\circ} \mathrm{C} \quad$ hold

$40 \mu 1$

The RT reaction was carried out in a PTC-100 programmable thermal controller (MJ Research Inc). Following the reaction, $40 \mu \mathrm{l}$ of ${ }_{\mathrm{NF}} \mathrm{H}_{2} \mathrm{O}$ was added to each PCR tube and cDNA was stored at $-20^{\circ} \mathrm{C}$. 


\section{$\underline{\text { SYBR Green Real-Time PCR }}$}

Every experiment was carried out under sterile conditions using filter tips (Rainin) and sterile $\mathrm{NF}_{2} \mathrm{O}$. Primers were reconstituted at a working stock concentration of $100 \mu \mathrm{M}$ and stored at $-20^{\circ} \mathrm{C}$. Primer mixes containing $4.5 \mu \mathrm{M}$ of forward primer and $4.5 \mu \mathrm{M}$ of reverse primer in $\mathrm{NFH}_{2} \mathrm{O}$ were prepared. The appropriate volume of master mix was prepared making the final concentration of each primer $0.3 \mu \mathrm{M}$. The following volumes represent one PCR reaction:

$\begin{array}{ll}\text { 1x Reaction mix: } & \\ \text { SYBR Green (Applied Biosystems) } & 12.5 \mu 1 \\ \text { Primer mix } & 1.7 \mu 1 \\ \mathrm{NF}_{2} \mathrm{O} & 9.3 \mu 1 \\ \text { Total } & 23.5 \mu 1\end{array}$

A $\beta$-actin real-time PCR reaction was prepared for every cDNA sample for each experiment performed. Each reaction was prepared in separate tubes, in duplicate, containing $2.5 \mu \mathrm{l} \mathrm{cDNA}$ or $\mathrm{NF}_{2} \mathrm{H}_{2} \mathrm{O}$ per reaction, and transferred to a Micro Amp Optical 96-well reaction plate (Applied Biosystems), $25 \mu 1$ per well. Plates were sealed with an optical adhesive cover (Applied Biosystems) using the applicator provided and a compression pad was placed on the plate before inserting into the machine. The PCR was carried out using the 7900HT Sequence Detection System from Applied Biosystems and samples were analysed using SDS 2.1 software.

\section{Taq Man Real-Time PCR}

Every experiment was carried out under sterile conditions using filter tips and sterile ${ }_{\mathrm{NF}} \mathrm{H}_{2} \mathrm{O}$. Primers and probes for TaqMan Real-Time PCR were purchased from Applied Biosystems. Primers were reconstituted at a working stock solution of $50 \mu \mathrm{M}$ and probes at a concentration of $10 \mu \mathrm{M}$ and stored at $-20^{\circ} \mathrm{C}$. $5 \mathrm{X}$ primer/probe mixes containing $4.5 \mu \mathrm{M}$ of forward primer, $4.5 \mu \mathrm{M}$ of reverse primer and $1.25 \mu \mathrm{M}$ probe in ${ }_{\mathrm{NF}} \mathrm{H}_{2} \mathrm{O}$ were prepared. 18S rRNA Taqman primer probe mix was purchased from Applied Biosystems. The appropriate volume of master mix was prepared making the final concentration of each primer $0.9 \mu \mathrm{M}$ and the final concentration of the probe at $0.25 \mu \mathrm{M}$. The following volumes represent one PCR reaction: 
1x Reaction mix

$\begin{array}{ll}\text { TaqMan Mastermix (Applied Biosystems) } & 12.5 \mu 1 \\ 5 \mathrm{X} \text { primer/probe mix } & 5 \mu 1 \\ 18 \mathrm{~S} \text { Probe (primer/probe) } & 1.25 \mu 1 \\ \mathrm{NFH}_{2} \mathrm{O} & 3.75 \mu 1 \\ \text { Total } & 22.5 \mu 1\end{array}$

Each reaction was prepared in separate tubes, in duplicate, containing $2.5 \mu 1$ cDNA or ${ }_{\mathrm{NF}} \mathrm{H}_{2} \mathrm{O}$ per reaction, and transferred to a Micro Amp Optical 96-well reaction plate, $25 \mu \mathrm{l}$ per well. The PCR was carried out using the $7900 \mathrm{HT}$ Sequence Detection System from Applied Biosystems and samples were analysed using SDS 2.1 software.

\subsection{MTT Assay}

The MTT assay measures cellular proliferation. Yellow MTT (3-(4,5dimethylthiazol-2yl)-2,5-diphenyl tetrazolium bromide) is reduced to purple formazan in the mitochondria of living cells. Dimethyl sulfoxide (DMSO) is added to dissolve the insoluble purple formazan product into a coloured solution, the absorbance of which can be measured at $\lambda 560 \mathrm{~nm}$ on a spectrophotometer. Primary mouse wild type and galectin- $3^{-/-}$HSCs were seeded at a density of 5,000 cells per well in a 96-well plate in complete media. Some galectin- $3^{-/-}$HSCs were incubated with $30 \mu \mathrm{g} / \mathrm{ml}$ galectin-3 over the time course. At 1, 2, 3, 6, 7 and 8 days a 1:10 dilution of $4 \mathrm{mg} / \mathrm{ml}$ MTT (Sigma-Aldrich) solution in PBS was added to the media $(0.4 \mathrm{mg} / \mathrm{ml}$ final concentration) after 4 hours, the media was removed and DMSO was added to solubilise the formazan. The purple solution was transferred to a 96-well plate and absorbance at $\lambda 560 \mathrm{~nm}$ was determined. The largest value was set to $100 \%$ growth and percentage growth was calculated.

\subsection{Cytokine Assays}

TNF- $\alpha$ and IL-6 cytokine release from BMDMs were measured using the Mouse Inflammation Cytometric Bead Array (CBA) kit (BD Biosciences) according to manufacturer's instructions. Along with IL-6 and TNF- $\alpha$ this kit can also be used 
to quantitatively measure IL-10, Monocyte Chemoattractant Protein-1 (MCP-1), IFN- $\gamma$ and IL-12p70 protein levels in a single sample. This study focused on TNF- $\alpha$ and IL-6 as the other cytokine concentrations were very low or undetectable. The kit contains six bead populations with distinct fluorescence intensities coated with capture antibodies specific for the above analytes. The six bead populations are mixed together to form the CBA which is resolved in the FL3 channel of a flow cytometer. The capture beads, PE-conjugated detection antibodies and recombinant standards or test samples are incubated together to form sandwich complexes. This study used a 96-well format of this assay which could be analysed using a BD FACS Array and FACS Array software.

A TGF- $\beta$ Quantikine Enzyme-linked Immuno Sorbent Assay (ELISA) kit $(\mathrm{R} \& \mathrm{D})$ was used to detect TGF- $\beta$ concentrations in supernatants recovered from day 4 wild type and galectin- $3^{-/-}$HSCs cultured for 24 hours in serum free media according to manufacturer's instructions. Briefly, standards, controls and samples are pipetted into a plate pre-coated with a monoclonal antibody specific to TGF- $\beta 1$. Any TGF- $\beta 1$ present is bound by the immobilised antibody. After washing away any unbound substances, an enzyme-linked polyclonal antibody specific for TGF- $\beta 1$ is added to the wells to sandwich the TGF- $\beta 1$ immobilised during the first incubation. Following a wash to remove any unbound antibody-enzyme reagent, a substrate solution is added to the wells and a colour develops in proportion to the amount of TGF- $\beta 1$ bound in the initial step. The colour development is stopped and the intensity of the colour is measured. Prior to the assay latent TGF- $\beta 1$ in the samples was activated to the immunoreactive TGF- $\beta 1$ detectable by the assay by the addition of $20 \mu 1$ of $1 \mathrm{~N} \mathrm{HCl}$ to $100 \mu 1$ culture supernatant for 10 minutes at room temperature. The acidified sample was neutralised by adding $13 \mu 1$ of $1.2 \mathrm{~N} \mathrm{NaOH} / 0.5 \mathrm{M}$ HEPES and assayed immediately.

\subsection{Nitrite Assay}

Nitric oxide (NO) production was determined using the Griess reaction which measures the stable oxidation product nitrite. Griess reagent (Sigma-Aldrich) was reacted in a 1:1 ratio with sample and measuring absorbance at $\lambda 560 \mathrm{~nm}$. A standard 
curve for nitrite was prepared by reacting known concentrations of sodium nitrite in the Griess reaction and measuring absorbance at $\lambda 560 \mathrm{~nm}$.

\subsection{Arginase Assay}

This method utilises the technique optimised by Corraliza and colleagues for assaying arginase activity (Corraliza et al., 1994). $2 \times 10^{5}$ peritoneal or bone marrow derived macrophages were seeded in 96-well plates and treated as indicated. The supernatants were removed and the cells were lysed for 15 minutes at $4^{\circ} \mathrm{C}$ in $100 \mu 1$ lysis buffer containing 0.1\% Triton X-100, a 1:50 dilution of EDTA-free protease inhibitor solution (50x stock is one Complete ${ }^{\mathrm{TM}}$ EDTA-free protease inhibitor tablet (Roche) per $1 \mathrm{ml}$ of PBS), and $1 \mathrm{mM}$ sodium orthovanadate. $50 \mu 1$ of lysate was then treated with $50 \mu 110 \mathrm{mM} \mathrm{MnCl}_{2}, 50 \mathrm{mM}$ Tris- $\mathrm{HCl} \mathrm{pH} 7.5$ for 10 minutes at $55^{\circ} \mathrm{C}$ $\left(\mathrm{MnCl}_{2}\right.$ is an essential cation for arginase activity). Arginine hydrolysis was carried out in eppendorf tubes and was initiated by the addition of $25 \mu 1$ of $0.5 \mathrm{M} \mathrm{L}$-arginine ( $\mathrm{pH} 9.7$ ) to a $25 \mu \mathrm{l}$ aliquot of the previously activated lysate for 1 hour at $37^{\circ} \mathrm{C}$. The reaction was stopped by the addition of $400 \mu \mathrm{l}$ of an acid mixture containing $\mathrm{H}_{2} \mathrm{SO}_{4}$, $\mathrm{H}_{3} \mathrm{PO}_{4}$ and $\mathrm{H}_{2} \mathrm{O}$ (1:3:7). The urea formed was quantified after the addition of $25 \mu 1$ $\alpha$-isonitrosopropiophenone (ISPF) dissolved in $100 \%$ ethanol and heating at $100^{\circ} \mathrm{C}$ for 45 minutes. The OD at $\lambda 560 \mathrm{~nm}$ was determined spectrophotometrically using $200 \mu 1$ aliquots in a 96-well plate. A standard curve was prepared with increasing amounts of urea (between 0.625 and $10 \mathrm{mM}$ ). In this case $100 \mu 1$ of urea solution, $400 \mu 1$ of the acid mixture and $25 \mu$ ISPF were added and the procedure followed as described above. Results are expressed as $\mu \mathrm{m}$ arginase activity/mg protein/hour.

\subsection{Antibacterial Assay}

Streptococcal pneumoniae (S. pn) was incubated overnight on a blood agar plate at $37^{\circ} \mathrm{C} / 5 \% \mathrm{CO}_{2}$. $10 \mathrm{ml}$ of $100 \%$ heart infusion broth (Difco) was inoculated with one colony of $S$. pn and incubated overnight at $37^{\circ} \mathrm{C}$ with gentle shaking. Cultures were centrifuged at 3000rpm for 20 minutes, washed with broth and centrifuged again. The culture was resuspended in broth to an $\mathrm{OD}_{600}=0.1$. The $S$. pn were diluted 1:100 (this was the bacterial reaction mix). $75 \mu 1$ of the bacterial 
reaction mix was incubated with $75 \mu 1$ antibiotic at $37^{\circ} \mathrm{C}$ for 2 hours at $800 \mathrm{rpm}$ on the thermomixer. Antibiotics used were: recombinant mouse galectin-3 (final concentrations $6.25,12.5,25,50$ and $100 \mu \mathrm{g} / \mathrm{ml}$ ), galectin-3 denatured for $10 \mathrm{mins}$ at $95^{\circ} \mathrm{C}$ (final concentrations $6.25,12.5,25,50$ and $100 \mu \mathrm{g} / \mathrm{ml}$ ) and ampicillin $(20 \mu \mathrm{g} / \mathrm{ml})$. A reaction was prepared for $\mathrm{t}=0$ which was plated out before the 2 hour incubation. After 2 hours, the reactions were serially diluted into $900 \mu 1 \mathrm{PBS}$ and $100 \mu 1$ of each dilution was plated out on blood agar plates. Plates were incubated overnight at $37^{\circ} \mathrm{C} / 5 \% \mathrm{CO}_{2}$ and colonies counted the next day.

\subsection{FITC-labelling of bacteria}

S. $p n$ and E. coli were incubated overnight on blood agar plates or LB agar culture plates respectively at $37^{\circ} \mathrm{C} / 5 \% \mathrm{CO}_{2}$. One colony of $S$. pn was inoculated into $10 \mathrm{ml}$ Todd Hewitt Broth (Oxoid) (containing 0.5\% yeast extract and 5\% FBS) and one colony of E. coli was inoculated into $10 \mathrm{ml}$ LB. Cultures were incubated at $37^{\circ} \mathrm{C} / 5 \% \mathrm{CO}_{2}$ for $4-5$ hours until they reached an $\mathrm{OD}_{660}$ of 0.5-0.6. Cultures were heat inactivated for 1 hour at $60^{\circ} \mathrm{C}$ in a water bath and heat inactivation confirmed by plating an aliquot onto agar plates. Heat inactivated cultures were centrifuged for 10 minutes at $2500 \mathrm{xg}$ to pellet bacteria and excess media was removed. The pellet was resuspended to an $\mathrm{OD}_{660}$ of 1.0 (approximately $1 \times 10^{9}$ bacteria per ml) with cold PBS, transferred to $1 \mathrm{ml}$ eppendorf tubes and centrifuged for 10 minutes at 2500rpm. Pellets were washed once with HBSS plus $1 \%$ BSA, centrifuged and supernatant removed. Bacterial pellets were resuspended in $100 \mu 10.5 \mathrm{mg} / \mathrm{ml}$ FITC solution (Sigma-Aldrich) in PBS, mixed thoroughly and rolled for 30 minutes at $4{ }^{\circ} \mathrm{C}$ in the dark. $900 \mu 1$ ice cold HBSS plus $1 \%$ BSA was added to each eppendorf and centrifuged for 2 minutes at 10krpm. Each pellet was washed twice with $1 \mathrm{ml}$ HBSS plus $1 \%$ BSA and resuspended in $1 \mathrm{ml}$ HBSS plus 1\% BSA. Labelled bacteria were aliquoted and stored at $-80^{\circ} \mathrm{C}$.

\subsection{Trypan Blue Viability Assay}

Viability of BMDM treated with either $S$. pn or E. coli was determined using Trypan Blue exclusion. Trypan blue is a stain that traverses only membranes of dead 
cells and colours these cells blue. Cells are washed with PBS, incubated in 2\% trypan blue solution for 1-2 minutes, washed twice with PBS to remove any nonabsorbed trypan blue then incubated with DMSO to solubilise the absorbed trypan blue. The absorbance at $\lambda 560 \mathrm{~nm}$ was measured for each sample. Methanol treatment of untreated wild type and galectin- $3^{-/-}$BMDM, before the addition of trypan blue, was used as an indication of total cell count and percentage cell death of each experiment was calculated.

\subsection{Preparation of human neutrophils from peripheral blood}

Blood was collected in tubes containing sodium citrate, at $1 \mathrm{ml}$ sodium citrate $/ 10 \mathrm{ml}$ blood, and mixed gently. Blood samples were centrifuged at $1250 \mathrm{rpm}$ for 20 minutes at $20^{\circ} \mathrm{C}$. The top platelet rich plasma layer was removed and $6 \%$ Dextran (GE Healthcare) added to sediment out the red blood cells $(2.5 \mathrm{ml}$ dextran/10ml cell pellet was added and made up to $50 \mathrm{ml}$ with $\mathrm{NaCl}$ ). After 30 minutes, the white blood cell layer was transferred to a $50 \mathrm{ml}$ conical tube and centrifuged at 1250rpm for 6 minutes. The supernatant was removed and the cells were gently resuspended. Cells were spun on a gradient made up of $81 \%, 68 \%$ and $55 \%$ Percoll layers at 1750rpm for 20 minutes with acceleration and brake off and granulocytes were harvested from in between the $81 \%$ and $68 \%$ layers. Neutrophils were washed in HBSS and counted on a haemocytometer. When needed, apoptotic human neutrophils were prepared by culturing neutrophils for 24 hours in IMDM containing $10 \%$ autologous serum.

\subsection{Phagocytosis Assays}

For FACS analysis of phagocytosis of bacteria by BMDM, wild type and galectin- $3^{-/-}$BMDM were seeded in 6-well plates at $2 \times 10^{5}$ cells/well, and left to adhere overnight in serum-free media. FITC-S. pn and FITC-E. coli were opsonised with $10 \%$ mouse serum for 1 hour at $37^{\circ} \mathrm{C}$. A 10:1 ratio of opsonised FITC-S. pn or FITC-E. coli to BMDM was incubated for 1 hour at $37^{\circ} \mathrm{C}$ or as indicated. Excess bacteria were washed off and cells were incubated with 5mM EDTA on ice for 15 
minutes. Cells were gently agitated off the bottom of the well and percentage phagocytosis determined by FACS analysis using a BD FacsCalibur flow cytometer.

For confocal analysis of phagocytosis of bacteria by macrophages, wild type and galectin- $3^{-/-}$BMDM (or alveolar macrophages) were seeded on coverslips at $4 \times 10^{5}$ cells/well, and left to adhere overnight in serum-free media. FITC-S. $p n$ and FITC-E. coli were opsonised with $10 \%$ mouse serum for 1 hour at $37^{\circ} \mathrm{C}$. A $10: 1$ ratio of opsonised FITC-S. pn or a 20:1 ratio of FITC-E. coli to BMDM was incubated for 1 hour at $37^{\circ} \mathrm{C}$, excess bacteria were washed off and cells were fixed in $3 \%$ PFA for 5 minutes at room temperature. Cells were blocked in 3\% BSA, containing 10\% mouse serum, for 1 hour at room temperature, incubated with 1:500 rhodamine phalloidin (Invitrogen) for 20 minutes at room temperature protected from light. To stain the nuclei $0.1 \mu \mathrm{g} / \mathrm{ml}$ DAPI in PBS was added for 5 minutes and coverslips were mounted onto slides (Fisher Scientific) using Mowiol. Images were taken using a Leica SP5 confocal microscope and subsequently processed using Adobe Photoshop.

For FACS analysis of phagocytosis of apoptotic human neutrophils by BMDM, BMDM were seeded in 48 -well plates, stained with $1 \mu \mathrm{g} / \mathrm{ml}$ Cell Tracker Orange (CMTMR, Invitrogen) and incubated with 10:1 ratio of $1 \mu \mathrm{g} / \mathrm{ml}$ Cell Tracker Green (CMFDA, Invitrogen) labelled day 1 apoptotic human neutrophils for up to 1 hour at $37^{\circ} \mathrm{C}$. Excess neutrophils were washed off and BMDMs were incubated with $5 \mathrm{mM}$ EDTA on ice for 15 minutes. Cells were gently agitated off the bottom of the well and percentage phagocytosis determined by FACS analysis using a BD FacsCalibur flow cytometer.

For confocal analysis of phagocytosis of apoptotic mouse neutrophils by BMDM, BMDM were seeded on coverslips, stained with Cell Tracker Orange and incubated with 5:1 ratio of Cell Tracker Green-labelled day 2 apoptotic wild type mouse neutrophils for 1 hour at $37^{\circ} \mathrm{C}$. Excess neutrophils were washed off and cells were fixed in 3\% PFA for 5 minutes at room temperature. To stain the nuclei $0.1 \mu \mathrm{g} / \mathrm{ml}$ DAPI in PBS was added for 5 minutes and coverslips were mounted using Mowiol. Pictures were taken using a Leica SP5 confocal microscope and subsequently processed using Adobe Photoshop. 
For FACS analysis of phagocytosis of bacteria by neutrophils, wild type and

galectin- $3^{-/-}$bone marrow derived neutrophils were incubated with a 10:1 ratio of opsonised FITC-S. $p n$ and FITC-E. coli for 1 hour at $37^{\circ} \mathrm{C}$, excess bacteria were washed off and percentage phagocytosis determined by FACS analysis using a BD FacsCalibur flow cytometer.

\subsection{Neutrophil Activation Assays}

\subsubsection{Neutrophil Adhesion Molecule Expression}

This assay was originally developed by Professor Adriano Rossi (University of Edinburgh) to examine the expression of adhesion molecules on neutrophil cell surfaces from human whole blood. For this thesis, the assay was employed to measure adhesion molecule expression on neutrophils from human whole blood and modified by myself to assay mouse whole blood. Upregulation of CD11b and shedding of CD62L are indicators of neutrophil activation. Agonists used were NFormyl-Methionine-Leucine-Phenylalanine (fMLP) $\quad(0.1 \mu \mathrm{M}), \quad$ LPS $\quad(100 \mathrm{ng} / \mathrm{ml})$ (R\&D) and galectin-3 (up to $30 \mu \mathrm{g} / \mathrm{ml}$ ). Whole human blood was collected in a $15 \mathrm{ml}$ conical tube with $10 \mathrm{U} / \mathrm{ml}$ heparin. Blood was added to the agonists in $2 \mathrm{ml}$ eppendorf tubes and incubated at $37^{\circ} \mathrm{C}$ for 30 minutes. Tubes were then placed on ice for 15 minutes and antibodies were added to separate $1.5 \mathrm{ml}$ eppendorfs and placed on ice. Antibodies used were anti-human CD11b-APC (clone ICRF44) (1:16.7) and antihuman CD62L-PE (clone DREG-56) (1:16.7). 50 4 l blood/agonist mixture was added to the relevant antibody mixture and incubated for 30 minutes at $4{ }^{\circ} \mathrm{C}$. Samples were treated with 1ml 1X FACS Lysing Solution (Becton Dickinson) for 5 minutes at room temperature to lyse the red blood cells and centrifuged at 300xg at $4^{\circ} \mathrm{C}$ for 5 minutes. The pellet was washed with $1 \mathrm{ml}$ PBS and centrifuged again. The pellet was resuspended in $300 \mu$ of $1 \%$ PFA in buffer (PBS and $0.1 \%$ BSA). Samples were analysed using the BD FACSCalibur flow cytometer and neutrophils were identified by forward and side scatter properties. The same method was carried out with wild type and galectin- $3^{-/-}$mouse whole blood. Approximately $1 \mathrm{ml}$ of mouse whole blood was collected from the vena cava of mice anaesthetised with avertin. Whole blood was incubated with anti-GR-1-PE antibody (clone RB6-8C5) 
(1:400) and analysed by FACS analysis to confirm the neutrophil population in the mouse blood. Mouse whole blood was incubated with $30 \mu \mathrm{g} / \mathrm{ml}$ galectin-3 followed by anti-mouse CD11b-APC (clone M1/70) (1:66.7) antibody. Events within the GR1 positive population were gated on.

\subsubsection{Superoxide Anion Generation Assay}

Cytochrome $\mathrm{C}$ molecules are reduced by the reactive oxygen species (ROS) $\mathrm{O}_{2}^{-}$resulting in a change in absorbance which can be measured spectrophotometrically (Dahlgren and Karlsson, 1999). There is a one-to-one molar stoichiometry between $\mathrm{O}_{2}^{-}$molecules and cytochrome $\mathrm{C}$ molecules reduced.

Neutrophils isolated from human peripheral blood were resuspended in HBSS $(+)$ containing calcium $\left(\mathrm{Ca}^{2+}\right)$ and magnesium $\left(\mathrm{Mg}^{2+}\right)$ to give a final concentration of $11.1 \times 10^{6}$ to $22.2 \times 10^{6}$ cells $/ \mathrm{ml}-$ used $90 \mu \mathrm{l}\left(1 \times 10^{6}\right.$ to $2 \times 10^{6}$ cells $)$ per tube). Neutrophils were primed with $1 \mu \mathrm{M}$ platelet-activating factor (PAF), HBSS $(+)$ or varying concentrations of galectin-3 for 15 minutes at $37^{\circ} \mathrm{C}$. Cells were then activated with $\operatorname{HBSS}(+)$ or fMLP $(0.1 \mu \mathrm{M})$ in $1 \mathrm{mg} / \mathrm{ml}$ warm cytochrome $\mathrm{C}$ for 15 minutes and centrifuged for 2 minutes at $13 \mathrm{krpm}$. Supernatants were transferred to fresh tubes and stored at $4^{\circ} \mathrm{C}$. Colour change was detected using a scanning spectrophotometer $500 \mathrm{~nm}-600 \mathrm{~nm}$, peak height was measured at $550 \mathrm{~nm}$. Absorbance was converted to concentration using the calculation $\mathrm{A}=\Sigma$.l.c where " $A$ " is the absorbance at $550 \mathrm{~nm}$ and " $\Sigma$ " is the extinction coefficient. The $\mathrm{OD}_{550}$ of the reaction mixture is converted to nmoles of cytochrome $\mathrm{C}$ reduced using the extinction coefficient $21.1 \times 10^{-3} \mathrm{M}^{-1} \cdot \mathrm{cm}^{-1}$. "l" is the length of light path $(1 \mathrm{~cm})$ and "c" is the concentration of absorbing substance in nmoles $/ \mathrm{ml} /$ number of neutrophils. Therefore $\mathrm{c}=\mathrm{A} /(\Sigma .1)$. With a light path of $1 \mathrm{~cm}$ and a sample volume of $1 \mathrm{ml}$, the conversion factor is $47.6\left(1 / 21.1 \times 10^{-3}\right)$ since $1 \mathrm{~mol}$ of $\mathrm{O}_{2}^{-}$reduced $1 \mathrm{~mol}$ of cytochrome C.

\subsubsection{Dihydrorhodamine (DHR) Activation}

DHR is a fluorescent dye which is activated by various reactive oxygen species. Neutrophils isolated from human peripheral blood were resuspended in PBS 
at $10 \times 10^{6}$ cells $/ \mathrm{ml}$. $100 \mu 1$ of neutrophils $\left(1 \times 10^{6}\right.$ cells $)$ were incubated with DHR $(0.1 \mathrm{mM})$ for 5 minutes at $37^{\circ} \mathrm{C}$ and primed with PAF $(1 \mu \mathrm{M})$ or human $(\mathrm{Hu})$ or mouse (Ms) galectin-3 $(10 \mu \mathrm{g} / \mathrm{ml})$ for 15 minutes at $37^{\circ} \mathrm{C}$. Cells were incubated with fMLP $(0.1 \mu \mathrm{M})$ for 15 minutes, $180 \mu$ l PBS added to increase the volume and placed on ice in the dark. DHR fluorescence was measured as a geo mean of FL-1 on the BD FacsCalibur flow cytometer.

\subsection{In vivo animal models}

\subsubsection{Chronic Carbon Tetrachloride $\left(\mathrm{CCl}_{4}\right)$ - induced liver injury model}

This model of liver fibrosis was originally developed by Professor John Iredale et al in rats. Dr Neil Henderson and I modified this protocol to use in mice and carried out the in vivo experiments together.

After overnight fast (with free access to water) age- and sex-matched mice were injected intraperitoneally (i.p.) with $1 \mu \mathrm{l} / \mathrm{g}$ body weight sterile $\mathrm{CCl}_{4}$ in a $1: 3$ ratio with sterile olive oil (Sigma-Aldrich) or olive oil (control) twice weekly for 8 weeks. The $\mathrm{CCl}_{4} /$ olive oil mixture was prepared fresh before each time point. Tissue was harvested 24 hours after the last injection of $\mathrm{CCl}_{4}$. Livers were harvested for analysis after 8 weeks and fixed in $10 \%$ buffered formalin or snap-frozen for subsequent real-time RT-PCR analysis (detailed in 2.10) or Western blot analysis. Formalin-fixed liver was paraffin embedded by the Histology department at the University of Edinburgh who also prepared $3 \mu \mathrm{m}$ thick sections of tissue onto superfrost slides using a microtome. $10 \mu \mathrm{m}$ sections were prepared for PSR staining.

\subsubsection{Model of Kidney Fibrosis using Unilateral Ureteric Obstruction (UUO)}

Experimental unilateral ureteric ligation resulting in UUO was kindly performed by Spike Clay (University of Edinburgh) as described previously (Kipari et al., 2006). UUO was performed by ligation of the left ureter of each animal at the ureteropelvic junction under anesthesia (i.p. injection of metatomidine and ketamine). The left flank area was shaved and the mouse was held into position with masking 
tape on its right side on a warm heating pad to ensure the shaved area was under minor tension. The shaved area was disinfected and $50 \mu 1$ of the analgesic buprenorphen was given subcutaneously. A longitudinal incision was made and the ureter was brought to the opening in the skin. The ureter was obstructed by applying a small titanium ligating clip (Weck 'Hemoclip Plus') and replaced in its original position. A 5-0 silk suture was used to close the peritoneal wall ensuring muscle and fat layers were included in the stitch and the skin was closed using a standard metal skin clip. The wound was disinfected with $10 \%$ Iodine solution and reversal agent (atipamezole) was injected subcutaneously. The mouse was given fluids by injecting $500 \mu 1$ saline into the scruff and allowed to recover. I harvested kidneys at days 3, 7 and 14 post obstruction with the help of Dr Alison Mackinnon and fixed in 10\% buffered formalin or methyl carnoys reagent or snap-frozen for subsequent real-time RT-PCR analysis (detailed above). Formalin- or methyl carnoys-fixed kidney was paraffin embedded by the Histology department at the University of Edinburgh who also prepared $3 \mu \mathrm{m}$ thick sections of tissue onto superfrost slides using a microtome. $10 \mu \mathrm{m}$ sections were prepared for PSR staining.

\subsubsection{Pneumonia model}

The method of intratracheal administration of bacteria to mice has been performed previously by Dr John Simpson and collegues (University of Edinburgh). Dr Neil Henderson and I planned and carried out the following experiment with the help of the collegues from Dr John Simpson's laboratory - Lesley Farrell (intratracheal administration of bacteria and bronchoalveolar lavages (BAL)) and Dr Tom Wilkinson (assisted with plating out samples for bacterial detection on agar plates).

S. pn type 3 was used in this study (ATCC - 10813). After overnight incubation on $5 \%$ sheep blood agar plates (BD), freshly grown colonies were suspended in heart infusion broth with 10\% heat treated horse serum (HIB-S) (Gibco, $\mathrm{UK}$ ) at an $\mathrm{OD}_{550}$ of 0.17 (PU8620 spectophotometer, Philips). The bacterial suspension was diluted 100-fold with fresh HIB-S and was incubated for 4 hours at $37^{\circ} \mathrm{C}$ with shaking, corresponding to a point compatible with logarithmic growth. The suspension was centrifuged at $3000 \mathrm{rpm}$ for 10 minutes at room temperature. 
The supernatant was discarded and the pellet was resuspended with the same volume of PBS. Serial dilutions were used for determination of exact bacterial concentration.

Escherichia coli (E. coli) (ATCC - 25922) were prepared using the above method but inoculated in LB broth instead of heart infusion broth and grown on LB agar plates instead of blood agar plates.

Galectin- $3^{-/-}$mice or wild type mice were used. Under anesthesia (avertin $10 \mu \mathrm{l} / \mathrm{g}$ body weight), mice were intratracheally (i.t.) inoculated with $1 \times 10^{5}$ colony forming unit (CFU) of $S$. pn or $1 \times 10^{7}$ CFU E. coli in $50 \mu 1$ volume. 15 hours after bacterial administration mice were sacrificed, blood collected aseptically, and bronchoalveolar lavage (BAL) was performed with two separate aliquots of $400 \mu 1$ sterile PBS as described in section 2.2. The right lung was fixed in $10 \%$ buffered formalin and the left lung stored in $2 \mathrm{ml}$ PBS for bacterial counts. BAL fluid and blood were serially diluted 10 -fold with PBS, inoculated on a $5 \%$ sheep blood agar plate (for mice infected with $S$. pn) or on an LB agar culture plate (for mice infected with E.coli), and incubated at $37^{\circ} \mathrm{C}$ overnight. The remaining BAL fluid was centrifuged at $3000 \mathrm{rpm}$ for 10 minutes at $4^{\circ} \mathrm{C}$. Protein concentration in BAL was determined by the BCA protein assay reagent according to manufacturer's instructions. The supernatant was stored at $-20^{\circ} \mathrm{C}$ until further use. The pellet was resuspended in $300 \mu 1$ of PBS, and total cell number was determined with a haemocytometer. For differential cell counts, cytospins were prepared (280rpm, 3 minutes at room temperature) and stained with Diff-Quick (Ragena) to enable the morphological discrimination between mononuclear cells and polymorphonuclear cells. The left lung was homogenized in $2 \mathrm{ml}$ of PBS and the homogenates were serially diluted 10 -fold with PBS, inoculated on 5\% sheep blood agar plates or LB agar plates, and incubated at $37^{\circ} \mathrm{C}$ overnight. Colonies formed from lung homogenates and BAL were counted and blood plates were examined for the presence (sepsis) or absence of colonies.

\subsection{Statistical analysis}

Results are reported as pooled data from a series of $\mathrm{n}$ separate experiments and presented as mean \pm Standard error mean (S.E.M.). Statistical significance was 
analysed by one-way analysis of variance (ANOVA) or two-tailed Student's $t$ test (Prism software). Statistical significance was assigned to data returning a $\mathrm{P}$ value of less than 0.05 . 


\section{Chapter 3}

\section{Purification of galectin-3 using the baculovirus expression system}

\subsection{Introduction}

The Bac-to-Bac ${ }^{\circledR}$ Baculovirus Expression System is used to generate highlevel expression of a gene of interest in insect cells. This method was chosen to overcome possible endotoxin contamination of recombinant galectin-3 when produced using the standard bacterial expression system.

This technique generates recombinant baculovirus by site-specific transposition in Escherichia coli (E. coli). The gene of interest is cloned into a pFastBac vector, containing a gentamycin resistance gene. This study uses the pFastBac HT vector supplied with the multiple cloning site in three reading frames (A, B and C) to enable cloning of the gene of interest in frame with the N-terminal 6x histidine (His) tag. The vector is then transformed into competent E. coli DH10Bac containing a baculovirus shuttle vector (bacmid) with a LacZ-mini-att $\operatorname{Tn} 7$ fusion and a kanamycin resistance marker. Transposition occurs between the miniTn7 element on the pFastBac vector and the mini-att $\operatorname{Tn} 7$ target site on the bacmid, generating a recombinant bacmid. A helper plasmid present in the bacteria provides the transposition proteins required for this reaction to take place. This helper plasmid also confers resistance to tetracycline.

This transposition disrupts the expression of the $L a c Z$ gene, so these bacteria can no longer produce the $\beta$-galactosidase enzyme encoded by this gene. 5-bromo-4chloro-3-indolyl- $\beta$-D-galactopyranoside $(\mathrm{X}$-gal) is included with isopropyl $\beta$-Dthiogalactoside (IPTG) (an inducer of $\beta$-galactosidase), kanamycin, tetracycline and gentamicin within the agar medium on the culture plate. $\mathrm{X}$-gal is cleaved by $\beta$ galactosidase yielding galactose and 5-bromo-4-chloro-3-hydroxyindole. 5-bromo4-chloro-3-hydroxyindole then is oxidized into 5,5'-dibromo-4,4'-dichloro-indigo, an insoluble blue product. Colonies containing the recombinant bacmid are white, whereas blue colonies contain the unaltered bacmid. White colonies are selected and 
re-streaked on culture plates to verify $E$. coli colonies containing recombinant bacmid.

The bacmid DNA contains M13 Forward (-40) and M13 Reverse priming sites flanking the mini-att $\operatorname{Tn} 7$ site to enable polymerase chain reaction (PCR) analysis of the recombinant bacmid (figure 3.1). Additionally, a combination of the M13 primers and primers directed to the insert can be used to verify the presence of the gene of interest.

Once confirmed to contain the gene of interest, the recombinant bacmid is transfected into insect cells using a cationic lipid transfection reagent to produce recombinant baculovirus. High transfection efficiency is determined by the following signs of infection: in the first 24 hours cells swell in diameter with increased size of cell nuclei, 24 to 72 hours cell growth ceases and there are signs of viral budding (granular appearance) and detachment. Cell lysis is observed after 72 hours. Once high transfection efficiency is demonstrated the medium containing the $\mathrm{P} 1$ baculovirus stock is collected and stored at $4^{\circ} \mathrm{C}$, protected from light, or at $-80^{\circ} \mathrm{C}$ for long-term storage.

Once isolated the P1 baculovirus is amplified to generate a higher-titre P2 stock. Titre of viral stock is determined using a 12-well End-point Dilution Assay (EPDA) which relies on visual comparison of signs of infection of cells inoculated with $100,10,1$ and $0 \mu 1$ of baculovirus. A high titre stock at $2 \times 10^{8}$ plaque forming units $(\mathrm{pfu}) / \mathrm{ml}$ shows equal signs of infection in all three $(100,10$ and $1 \mu \mathrm{l})$ infected wells 3 days post inoculation. This high titre stock is then used for large scale inoculation for production of recombinant protein.

Once infected with recombinant baculovirus the insect cells express Histagged recombinant protein through the cell's normal transcription/translation machinery which can then be purified using metal chelation chromatography. 
Figure 3.1 - Schematic diagram illustrating the M13 primer sites on the recombinant bacmid.

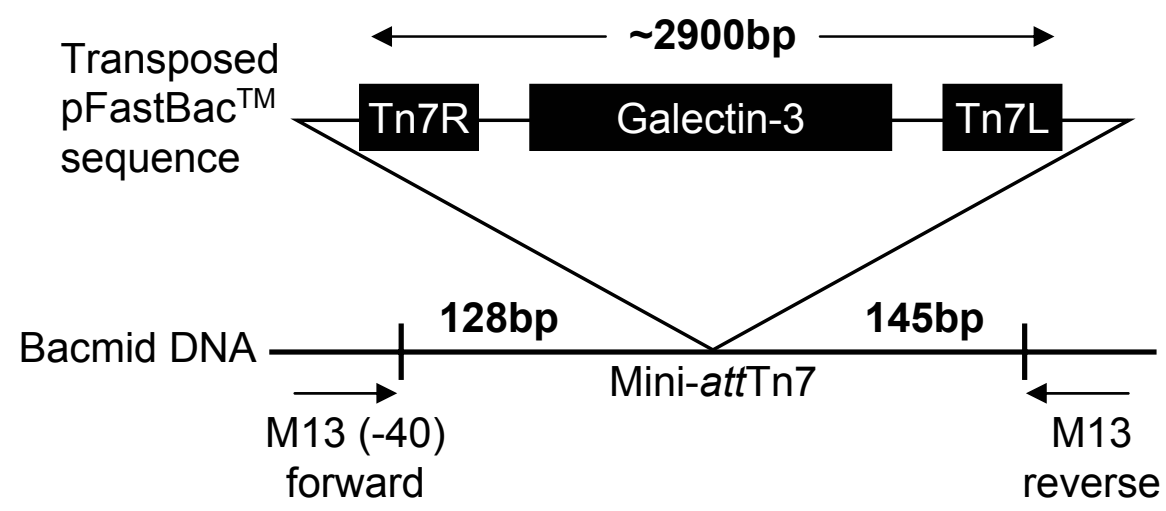

Figure 3.1

The bacmid DNA containing M13 forward (-40) and M13 reverse priming sites flanking the mini-att $\mathrm{Tn} 7$ site enabling PCR analysis of the recombinant bacmid. 


\subsection{Methods and Results}

\subsubsection{Generating the Recombinant pFastBac Vector}

The pFastBac HT vector was used in this study as it contains a $6 x H i s$ tag which was subsequently used to purify the recombinant protein. The pFastBac HT vector is supplied with the multiple cloning site in three reading frames (A, B and C) to enable cloning of the gene of interest in frame with the N-terminal 6xHis tag. Mouse galectin-3 was cloned into pFastBac HT A and human galectin-3 was cloned into pFastBac HT B to ensure the gene was in frame with the initiation ATG and the $\mathrm{N}$-terminal 6xHis tag (figure 3.2A-C).

Mouse galectin-3 was amplified from a pcDNA3.1 mouse galectin-3 clone, previously made in the laboratory using pcDNA3.1 vector (Invitrogen), using primers designed with a BamHI restriction site on the forward primer and an EcoRI restriction site on the reverse primer (see "Materials and Methods Chapter" for primer sequences). The following reaction conditions were used:

$\left.\begin{array}{llll}\text { pcDNA3.1 } & 1 \mu 1 & \text { Conditions } & \\ \text { dNTPs } & 1 \mu 1 & & \\ 10 x \text { buffer } & 5 \mu 1 & 95^{\circ} \mathrm{C} & 5 \mathrm{~min} \\ \text { Taq polymerase } & 0.5 \mu 1 & 95^{\circ} \mathrm{C} & 1 \mathrm{~min} \\ \text { Pfu }(1: 50) & 0.5 \mu 1 & 56^{\circ} \mathrm{C} & 1 \mathrm{~min} \\ \text { Forward primer }(100 \mathrm{pmol} / \mu 1) & 2 \mu 1 & 72^{\circ} \mathrm{C} & 1 \mathrm{~min} \\ \text { Reverse primer }(100 \mathrm{pmol} / \mu \mathrm{l}) & 2 \mu 1 & 72^{\circ} \mathrm{C} & 10 \mathrm{~min}\end{array}\right\} \quad 30 \mathrm{x}$

The mouse galectin-3 PCR product flanked by BamHI and EcoRI sites and pFastBac HT A were digested using BamHI and EcoRI restriction enzymes (Promega) for 2 hours at $37^{\circ} \mathrm{C}$. Digests were prepared as follows: 
Figure 3.2 - Maps of the pFastBac HT vectors.

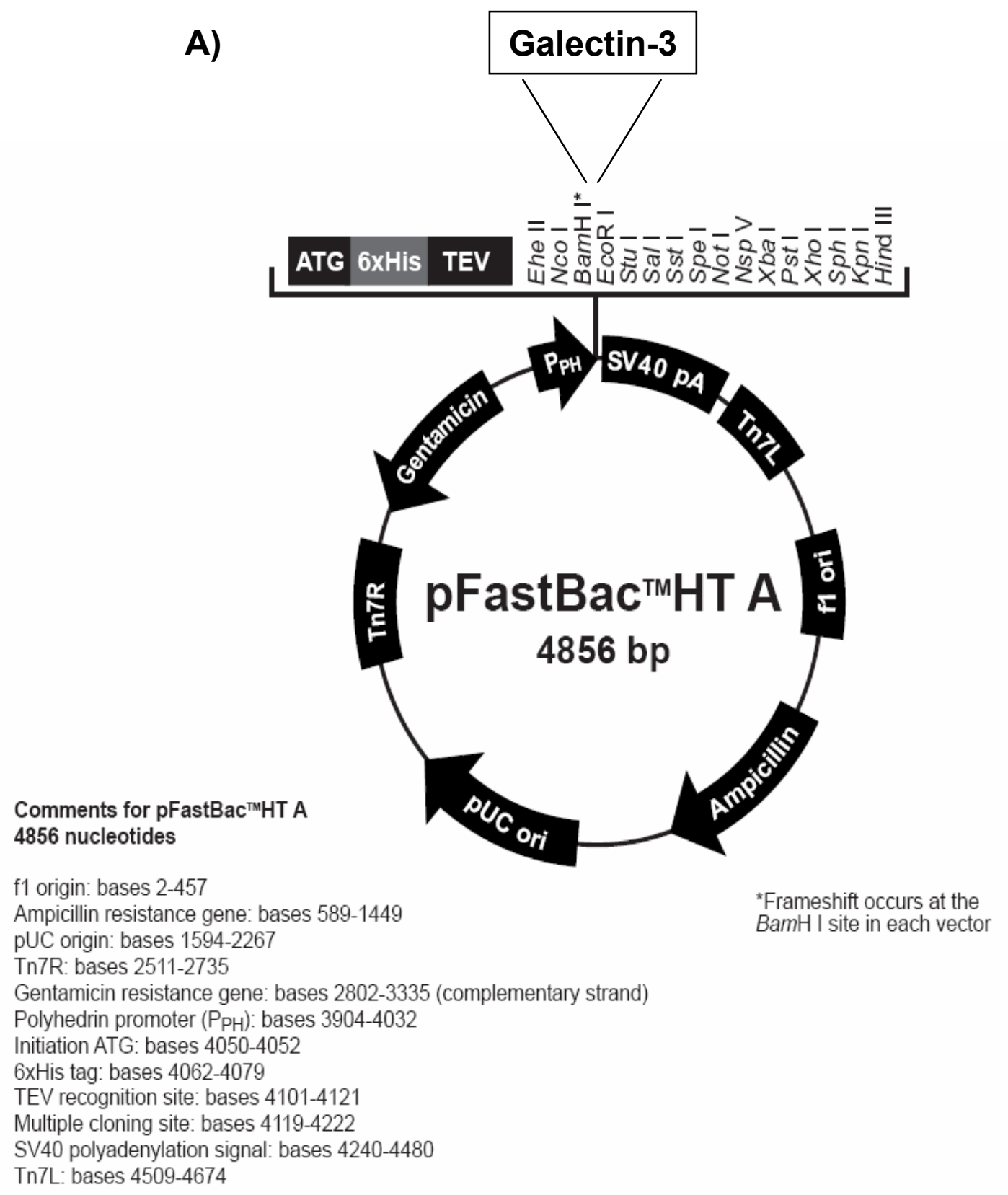

Figure 3.2 (A)

(A) The pFastBac HT vector used to clone galectin-3 in frame with the initiation ATG and the N-terminal 6xHis tag. 


\section{B) pFastBac HT A Polylinker}

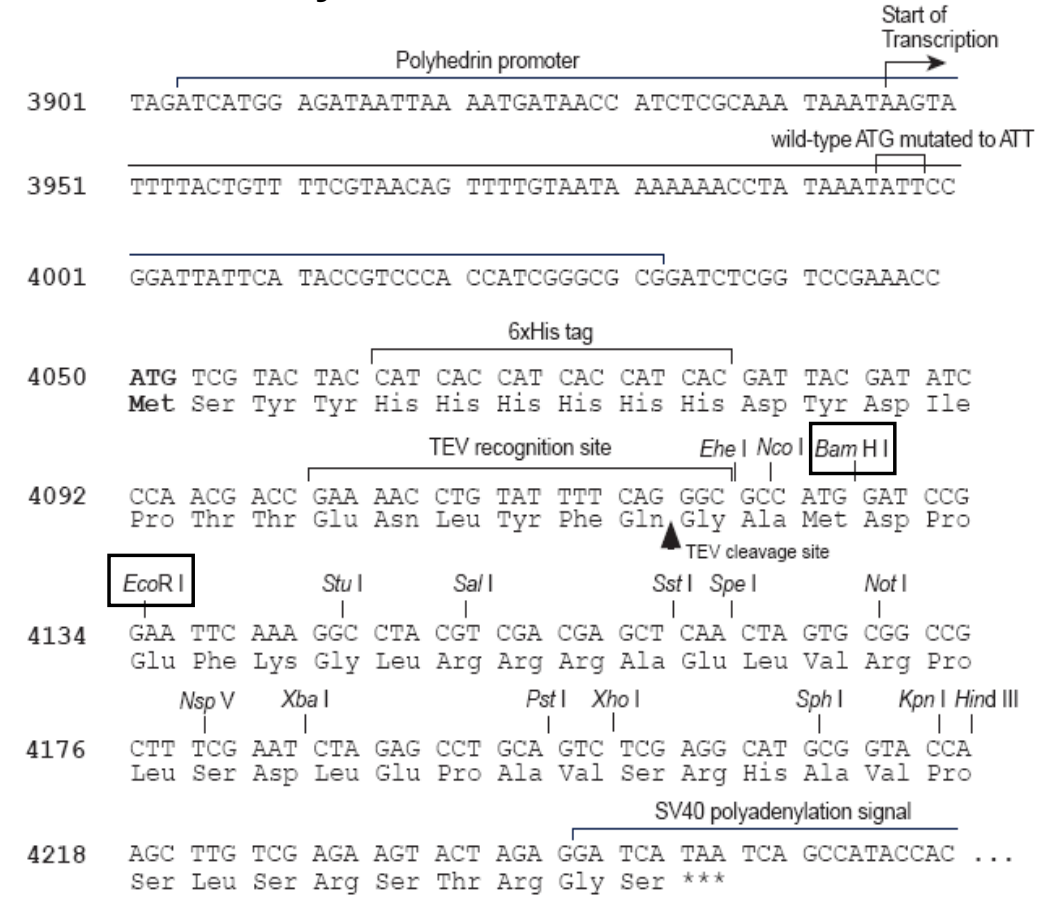

\section{C) pFastBac HT B Polylinker}

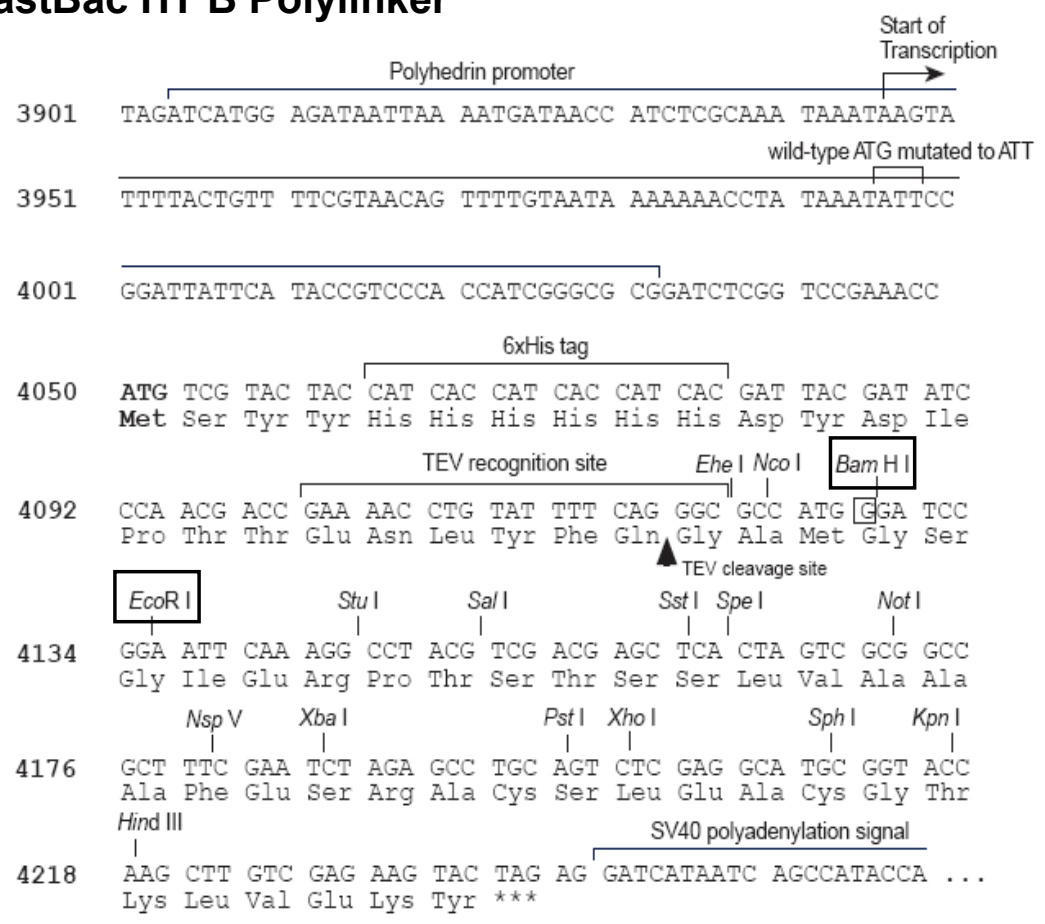

Figure 3.2 (B, C)

Multiple cloning site of (B) pFastBac HT A and (C) pFastBac HT B. Highlighted are the restriction sites used to clone in mouse and human galectin-3. 
Mouse galectin-3 PCR product

pFastBac HT A $(1 \mu \mathrm{g})$

10x Buffer E (Promega)

BamHI

EcoRI

${ }_{\mathrm{NF}} \mathrm{H}_{2} \mathrm{O}$

Total

$\begin{array}{cc}\text { Insert digest } & \text { pFastBac HT } \\ 24 \mu 1 & - \\ - & 2 \mu 1 \\ 3 \mu 1 & 3 \mu 1 \\ 1.5 \mu 1 & 1.5 \mu 1 \\ 1.5 \mu 1 & 1.5 \mu 1 \\ - & 22 \mu 1 \\ 30 \mu 1 & 30 \mu 1\end{array}$

The digests were run on a $1 \%$ agarose gel (figure 3.3A) and gel extracted using the QIA quick gel extraction kit (Qiagen) as per manufacturer's instructions.

Ligation of the digested pFastBac HT A vector and mouse galectin-3 insert was carried out overnight at $18^{\circ} \mathrm{C}$ as follows:

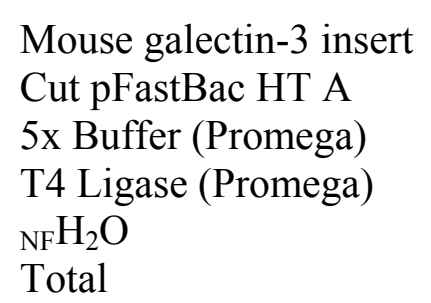

Ligation
$12 \mu 1$
$2 \mu 1$
$4 \mu 1$
$2 \mu 1$
-
$20 \mu 1$

Vector only control
-
$2 \mu 1$
$4 \mu 1$
$2 \mu 1$
$12 \mu 1$
$20 \mu 1$

For the remainder of this chapter this construct will be referred to as pFastBac MsG3.

A human galectin-3 insert was digested out of a pGEM-T Easy human galectin-3 clone, previously made in the laboratory using pGEM-T Easy Vector (Promega), and pFastBac HT B was cut using BamHI and EcoRI restriction enzymes. Digests were prepared as follows:

pGEM-T Easy galectin-3 pFastBac HT B $(1 \mu \mathrm{g})$

10x Buffer E

BamHI

EcoRI

${ }_{\mathrm{NF}} \mathrm{H}_{2} \mathrm{O}$

Total
pGEM-T Easy digest pFastBac HT B digest

$3 \mu 1$

$3 \mu 1$

$1.5 \mu 1$

$1.5 \mu 1$

$21 \mu 1$

$30 \mu 1$
$2 \mu 1$

$3 \mu 1$

$1.5 \mu 1$

$1.5 \mu 1$

$22 \mu 1$

$30 \mu 1$ 
Figure 3.3 - Restriction digests of the recombinant pFastBac HT Vectors.
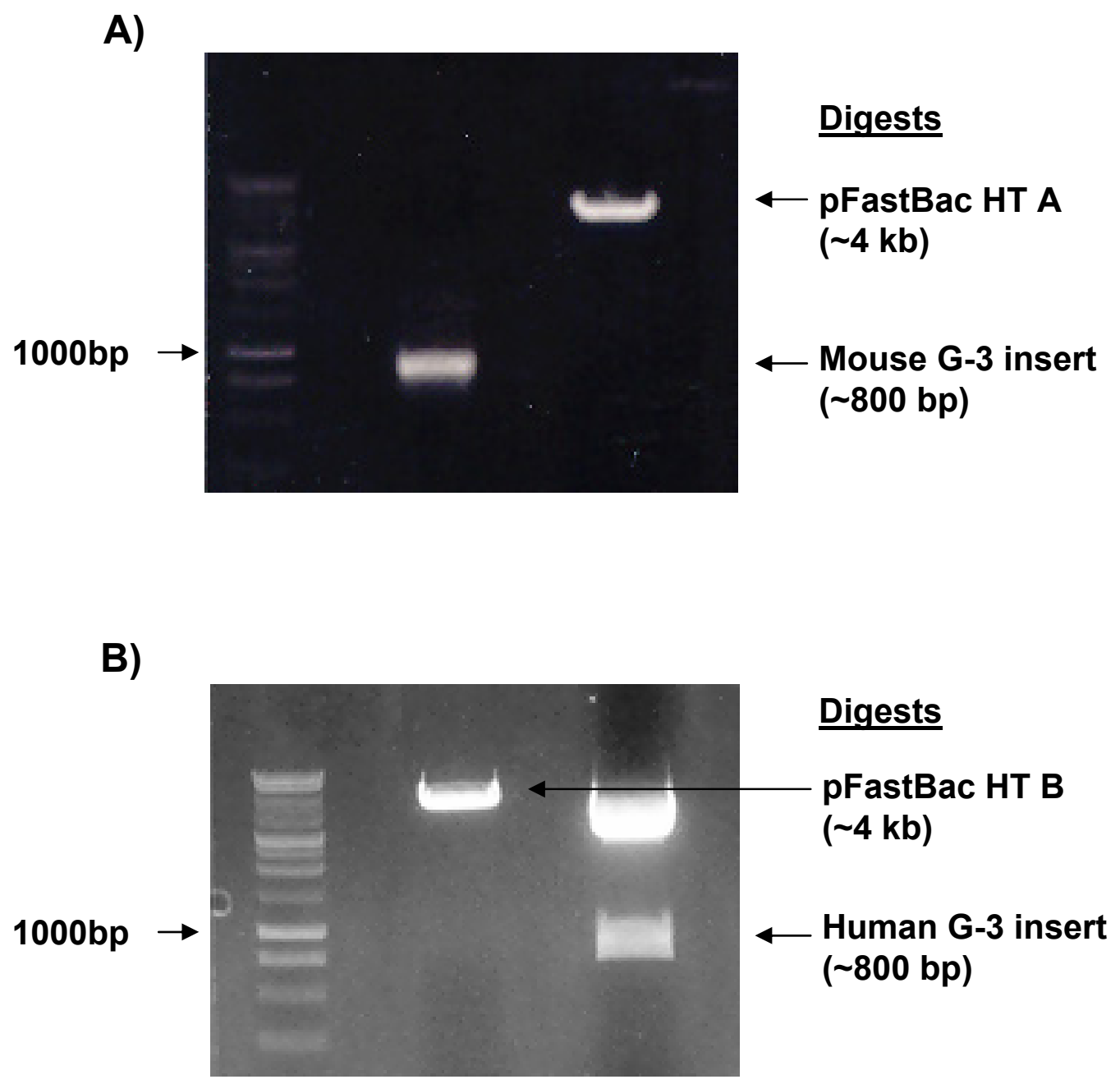

Figure 3.3 (A, B)

Restriction digests of (A) pFastBac HT A vector and the mouse galectin-3 insert and (B) pFastBac HT B vector and the human galectin-3 insert were carried out and run on $1 \%$ agarose gels. The relevant bands (indicated with arrows) were gel extracted and ligations were performed to make recombinant vector. 
The digests were run on a $1 \%$ agarose gel (figure $3.3 \mathrm{~B}$ ) and the relevant bands were gel extracted using the Qiagen gel extraction kit according to the manufacturer's instructions.

Ligation of the digested pFastBac HT B vector and human galectin-3 insert was carried out overnight at $18^{\circ} \mathrm{C}$ using the same procedure as detailed above. For the remainder of this chapter this construct will be referred to as pFastBac HuG3.

\subsubsection{Transformation of the E. coli strain TOP10 with recombinant vector}

To increase the yield of the recombinant vector, TOP10 competent $E$. coli (Invitrogen) were transformed and ampicillin-resistant transformants were selected.

$50 \mu 1$ of TOP10 bacteria were incubated on ice with $5 \mu 1$ of pFastBac MsG3, pFastBac HuG3, or vector only controls for 30 minutes. Tubes were placed at $42^{\circ} \mathrm{C}$ for 50 seconds to heat shock the bacteria and then immediately placed on ice for 2 minutes. $500 \mu \mathrm{l}$ S.O.C. was added to each tube and incubated for 50 minutes at $37^{\circ} \mathrm{C}$ with shaking at $750 \mathrm{rpm}$. The bacteria were centrifuged, $400 \mu 1$ of supernatant removed and the bacteria were resuspended in the remaining S.O.C. Bacteria were plated on Luria-Bertani (LB) agar plates containing $50 \mu \mathrm{g} / \mathrm{ml}$ ampicillin and incubated overnight at $37^{\circ} \mathrm{C}$. The next day, colonies were observed on culture plates which had been plated with bacteria transformed with the recombinant vectors indicating that the ligations between inserts and cut vectors were successful. There were no colonies seen on the negative control culture plates which had been plated with bacteria transformed with the vector only control ligation as predicted.

Separate colonies were selected using a sterile $200 \mu 1$ pipette tip which was then ejected into $3 \mathrm{ml} \mathrm{LB}$ plus ampicillin and incubated overnight at $37^{\circ} \mathrm{C}$. The next day, mini-preps were performed on $1.5 \mathrm{ml}$ of each suspension using the Promega 'Wizard' mini-prep kit according to manufacturer's instructions. Restriction digests were performed on each mini-prep using enzymes BamHI and EcoRI to check that the vectors contained the inserts, and samples were run on a $1 \%$ agarose gel (figure 3.4A and B). All digests were positive for the insert. 
Figure 3.4 - Restriction digests of mini-preps from transformed TOP10 bacteria.

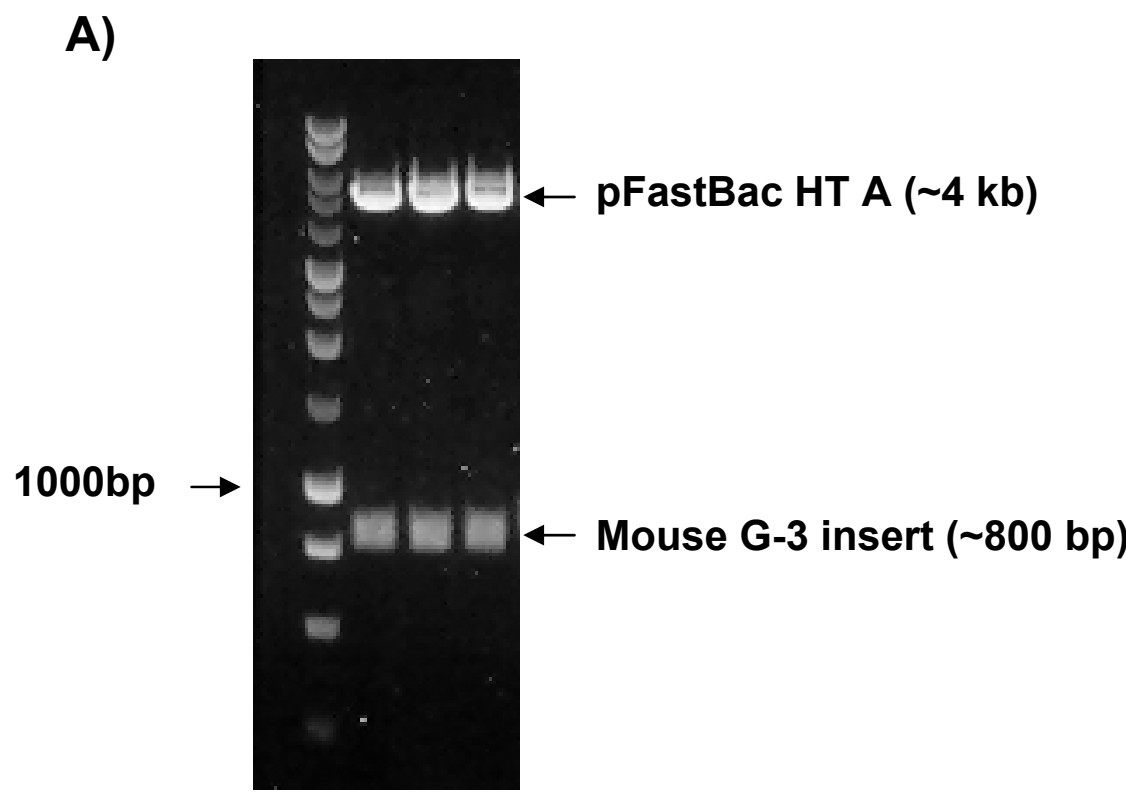

B)

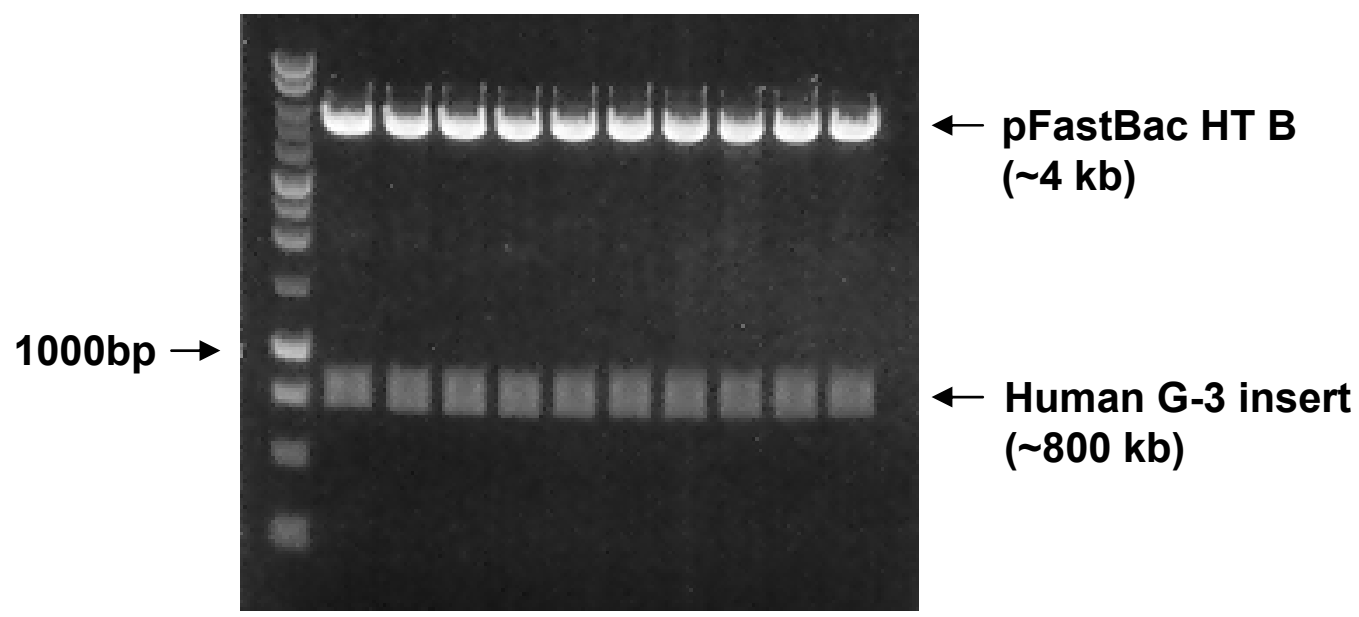

Figure 3.4 (A, B)

Restriction digests of mini-preps prepared from TOP10 bacteria transformed with (A) pFastBac MsG3 and (B) pFastBac HuG3 recombinant vector. 
The remaining $1.5 \mathrm{ml}$ of one starter culture of TOP10 bacteria containing pFastBac MsG3 and one starter culture of TOP10 bacteria containing pFastBac HuG3 were inoculated into $500 \mathrm{ml}$ LB plus ampicillin and incubated overnight at $37^{\circ} \mathrm{C}$. Maxi-preps were performed on each culture using the Qiagen maxi-prep kit according to manufacturer's instructions. DNA yields, determined using a WPA UV 1101 Biotech photometer, were $5.7 \mu \mathrm{g} / \mu \mathrm{l}$ of pFastBac MsG3 and $3.13 \mu \mathrm{g} / \mu 1$ of pFastBac HuG3.

\subsubsection{Generating Recombinant Bacmid}

The recombinant vector was then transformed into DH10Bac E. coli (Invitrogen) to generate recombinant bacmid. Transposition occurs between the mini-Tn7 element on the pFastBac vector and the mini-att $\operatorname{Tn} 7$ target site on the bacmid and disrupts the expression of the LacZ gene enabling blue/white selection to identify colonies containing recombinant bacmid.

$100 \mu 1$ of DH10Bac E. coli were transferred to pre-chilled $1.5 \mathrm{ml}$ tubes. 10ng of recombinant vector or 1 ng of control vector pFastBac HT-CAT, provided in the Bac-to-Bac kit, was incubated with the bacteria for 30 minutes at $4{ }^{\circ} \mathrm{C}$. The bacteria were heat shocked for 45 seconds at $42^{\circ} \mathrm{C}$ without shaking then transferred to ice for 2 minutes. $900 \mu \mathrm{l}$ S.O.C. was added to each tube and incubated for 4 hours at $37^{\circ} \mathrm{C}$ with shaking at 750rpm. Serial dilutions $(1: 10,1: 100$ and 1:1000) of the suspension were inoculated onto LB agar culture plates containing $50 \mu \mathrm{g} / \mathrm{ml}$ kanamycin, $7 \mu \mathrm{g} / \mathrm{ml}$ gentamicin (Gibco), $10 \mu \mathrm{g} / \mathrm{ml}$ tetracycline, $100 \mu \mathrm{g} / \mathrm{ml} \mathrm{X-gal} \mathrm{(Invitrogen)} \mathrm{and} 40 \mu \mathrm{g} / \mathrm{ml}$ IPTG to select for DH10Bac transformants. Culture plates were incubated for 48 hours at $37^{\circ} \mathrm{C}$.

Colonies containing the recombinant bacmid were white amongst a background of blue colonies containing the unaltered bacmid. E. coli transformed with the control vector were blue. White colonies were selected and re-streaked on culture plates to verify E. coli colonies with recombinant bacmid. Once verified, white colonies were selected and each colony inoculated into starter cultures of $10 \mathrm{ml}$ LB containing $50 \mu \mathrm{g} / \mathrm{ml}$ kanamycin, $7 \mu \mathrm{g} / \mathrm{ml}$ gentamicin and $10 \mu \mathrm{g} / \mathrm{ml}$ tetracycline overnight at $37^{\circ} \mathrm{C}$ with shaking. $2 \mathrm{ml}$ of each starter culture was inoculated into a 
larger volume of $500 \mathrm{ml} \mathrm{LB}$ plus antibiotics and incubated overnight at $37^{\circ} \mathrm{C}$ with shaking.

Recombinant bacmid was isolated using the S.N.A.P MidiPrep Kit (Invitrogen), which is designed for purifying highly pure plasmid DNA, according to manufacturer's instructions.

\subsubsection{Analysing Recombinant Bacmid DNA by PCR}

The bacmid DNA contains M13 Forward (-40) and M13 Reverse priming sites flanking the mini-att $\operatorname{Tn} 7$ site to enable PCR analysis of recombinant bacmid (figure 3.1). Once the gene of interest was inserted, a combination of the M13 primers and primers directed against the insert were used to verify the presence of the galectin-3 gene. The length of the transposed pFastBac sequence with the galectin-3 insert is approximately $2900 \mathrm{bp}$, and if transposition has occurred, the length of the PCR product using the M13 Forward (-40) and M13 Reverse primers is approximately $3200 \mathrm{bp}$. If transposition has not occurred, the length of the PCR product is only approximately $270 \mathrm{bp}$ so it is easy to distinguish which bacmids contain the galectin-3 gene.

Primer sequences are listed in Materials and Methods Chapter 2. 10x Titanium Taq PCR Buffer and 50x Titanium Taq DNA polymerase were from BD Biosciences. The following reaction conditions were used:

$\left.\begin{array}{llll}\text { Recombinant bacmid DNA (100ng) } & 1 \mu \mathrm{l} & \text { Conditions } & \\ \text { 10x Titanium Taq PCR Buffer } & 5 \mu \mathrm{l} & & \\ \text { 10mM dNTP mix } & 1 \mu \mathrm{l} & 93^{\circ} \mathrm{C} & 3 \mathrm{~min} \\ \text { 50x Titanium Taq DNA polymerase } & 1 \mu 1 & 94^{\circ} \mathrm{C} & 45 \mathrm{sec} \\ \text { Forward primer (10 } \mu \mathrm{M} \text { stock) } & 1.25 \mu 1 & 55^{\circ} \mathrm{C} & 45 \mathrm{sec} \\ \text { Reverse primer }(10 \mu \mathrm{M} \text { stock) } & 1.25 \mu 1 & 72^{\circ} \mathrm{C} & 5 \mathrm{~min} \\ \mathrm{NF}_{2} \mathrm{O} & 39.5 \mu 1 & 72^{\circ} \mathrm{C} & 7 \mathrm{~min}\end{array}\right\} 35 \mathrm{x}$

Combinations of primers used were as follows for PCR of both mouse galectin-3 bacmid and human galectin-3 bacmid. A bacmid prepared from a blue colony (untransformed) was also amplified with the M13 primers as a negative control: 
Figure 3.5 - PCR analysis of the recombinant bacmids.
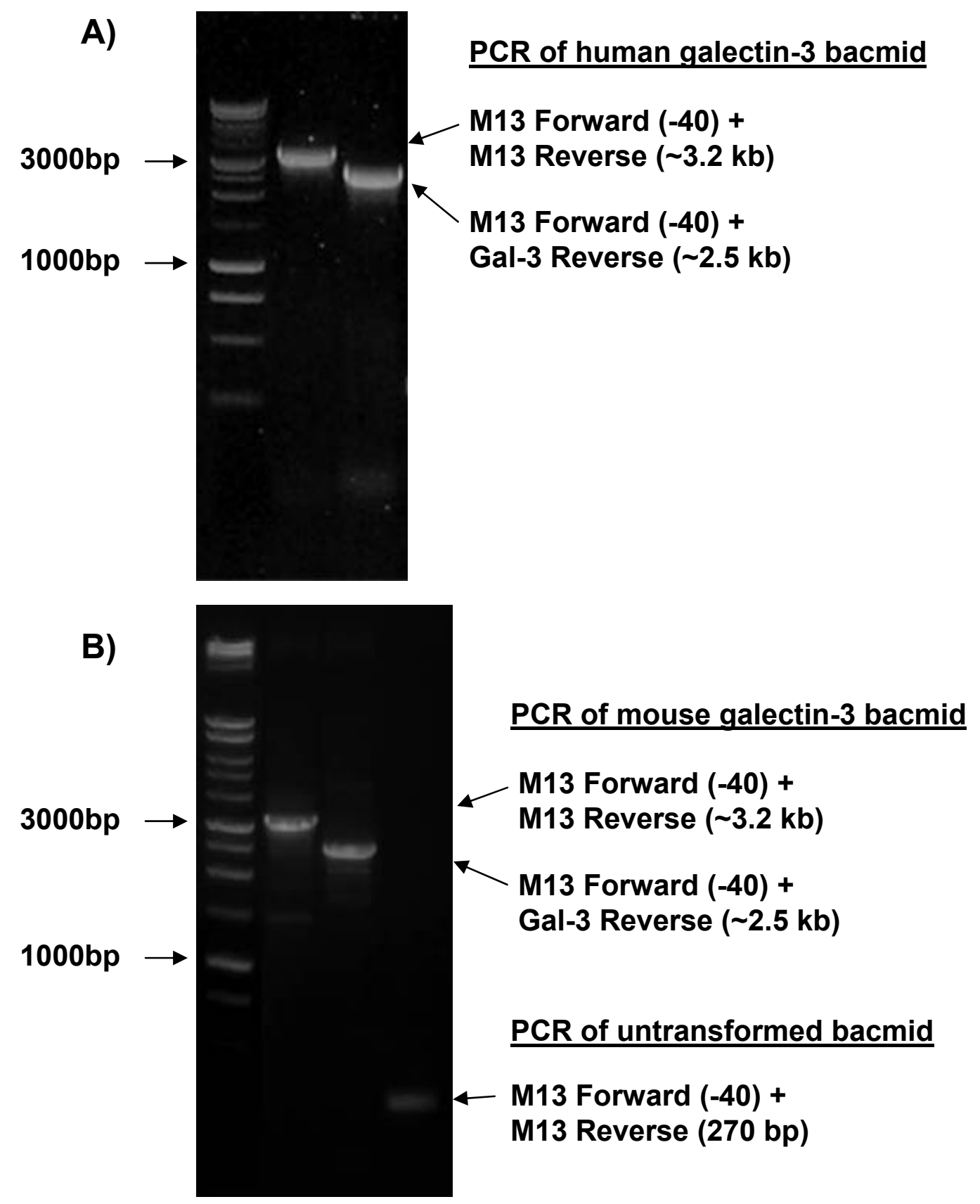

Figure 3.5 (A, B)

PCR of recombinant bacmid prepared from DH10Bac E. coli transformed with (A) pFastBac HT HuG3 recombinant vector and (B) pFastBac HT MsG3 recombinant vector or untransformed. 


\author{
Forward Primer Reverse Primer \\ M13 Forward (-40) M13 Reverse \\ M13 Forward (-40) Galectin-3 Reverse
}

Samples were run on a $1 \%$ agarose gel (figure 3.5) and show that the galectin-3 gene was present in both the mouse and human galectin-3 recombinant bacmids.

\title{
3.2.5 Transfecting Insect Cells with Recombinant Bacmids
}

Once confirmed to contain the galectin-3 gene for both human and mouse, the recombinant bacmids were transfected into Sf9 insect cells to produce recombinant baculovirus. The cationic lipid used to carry out the transfection was Cellfectin (Invitrogen), recommended for transfection of Sf9 and other insect cells. Grace's Insect Cell Culture Medium (Invitrogen) was used to increase transfection efficiency.

Cellfectin, Grace's medium and Sf-900 II serum-free growth medium were warmed up to room temperature before use. Sf9 cells were seeded in a 6-well culture plate at $9 \times 10^{5}$ cells/well in $2 \mathrm{ml}$ of growth medium, and left to adhere for at least 1 hour at $27^{\circ} \mathrm{C}$ in a humidified incubator. For each transfection the bacmid DNA:Cellfectin reagent complexes were prepared as follows in sterile $5 \mathrm{ml}$ polystyrene round-bottom tubes. $1 \mu \mathrm{g}$ of purified bacmid DNA was diluted in $100 \mu 1$ of unsupplemented Grace's medium. The Cellfectin reagent was mixed thoroughly and $6 \mu 1$ was diluted with $100 \mu 1$ unsupplemented Grace's medium. The diluted bacmid DNA was then combined with the diluted Cellfectin reagent, mixed gently and incubated for 30 minutes at room temperature. The growth media was removed from the Sf9 cells and cells were washed with unsupplemented Grace's media. After 30 minutes, $800 \mu \mathrm{l}$ unsupplemented Grace's medium was added to each tube containing the DNA:lipid complexes and mixed gently. Wash media was removed and the $\sim 1 \mathrm{ml}$ of DNA:lipid complexes were added to each well and incubated for 5 hours at $27^{\circ} \mathrm{C}$. DNA:lipid complexes were removed and $2 \mathrm{ml}$ of growth media was added to each well. Cells were incubated in a $27^{\circ} \mathrm{C}$ humidified incubator for 72 hours or until signs of viral infection became visible. Cellfectin-only negative 
controls and cells transfected with untransformed bacmid, positive controls, were also performed.

\subsubsection{Isolating and Amplifying Baculoviral Stocks}

Recombinant virus was released into the medium 72 hours after transfection. The medium was transferred into sterile $15 \mathrm{ml}$ snap-cap tubes, centrifuged for 5 minutes at 500xg to remove cell debris and clarified supernatants transferred to fresh tubes. Foetal bovine serum was added to a final concentration of $2 \%$ and the mouse galectin-3 and human galectin-3 P1 baculoviral stocks were stored at $4^{\circ} \mathrm{C}$ protected from light, with aliquots kept at $-80^{\circ} \mathrm{C}$ for long term storage. The titre of the P1 viral stock ranges from $1 \times 10^{6}$ to $1 \times 10^{7} \mathrm{pfu} / \mathrm{ml}$.

Baculoviral stocks were amplified three times to P2, P3 and P4. Sf9 cells were seeded in a 6 -well culture plate at $2 \times 10^{6} /$ well in $2 \mathrm{ml}$ of growth medium and left to adhere for at least 1 hour. $200 \mu 1$ of baculoviral stock was added to each well and incubated for 5 days at $27^{\circ} \mathrm{C}$ in a humidified incubator. Virus was isolated after 5 days using the technique described above.

Titre of the viral stocks was determined using a 12-well EPDA. Sf9 cells were seeded in a 12 -well culture plate at $1 \times 10^{5} /$ well in $1 \mathrm{ml}$ growth medium and left to adhere for at least 1 hour. Cells were inoculated with 100,10, 1 or $0 \mu 1$ of each baculoviral stock and incubated for 3 days at $27^{\circ} \mathrm{C}$ in a humidified incubator. After 3 days equal signs of infection were seen in all 3 infected wells of cells inoculated with P3 and P4 mouse galectin-3 or human galectin-3 baculovirus indicating that these were high titre stocks and could be used for subsequent inoculation of insect cells for protein production.

\subsubsection{Expressing Recombinant Protein}

Once suitable volumes of high titre baculoviral stocks had been produced, enough insect cells could be infected to produce enough recombinant protein to be used in future experiments. For expression of each protein, three $162 \mathrm{~cm}^{2}$ cell culture flasks were seeded with $3 \times 10^{7} \mathrm{Sf} 9$ cells in $30 \mathrm{ml}$ growth media and left to adhere for at least 1 hour. $1 \mathrm{ml}$ of high titre baculovirus was added to each flask and incubated 
for 5 days at $27^{\circ} \mathrm{C}$ in a humidified incubator. Cells from each flask were scraped and pelletted and supernatant removed. It was unclear whether galectin-3 would be released from the Sf9 cells into the growth medium and so initial experiments used both cell supernatant and cell lysate for protein purification. Results indicated much greater protein concentration in the cell lysate. Subsequent protein purification was therefore performed using cell lysate. Pellets from equivalent infections were pooled, lysed at room temperature for 5 minutes in $6 \mathrm{ml} \mathrm{Cytobuster} \mathrm{(Novagen)} \mathrm{and}$ split into $1.5 \mathrm{ml}$ tubes and centrifuged at $13 \mathrm{krpm}$ for 10 minutes at $4^{\circ} \mathrm{C}$. His-tagged recombinant protein was purified using the Ni-NTA His bind ${ }^{\circledR}$ resin as detailed in Chapter 2 (Materials and Methods). Flow through (FT), washes (W1-3) and elutions (E1-5) were collected and aliquots were analysed using Sodium dodececyl-sulphate polyacrylamide gel electrophoresis (SDS-PAGE) with protein bands stained with Simply Blue Safestain (Invitrogen) (figure 3.6). Elutions 2-5 were dialysed against 1X PBS (dialysis tubing size $-14.3 \mathrm{~mm}$ ). Protein determination using Pierce BCA protein assay reagent was performed and Western blots used to verify the presence of galectin-3 (figure 3.7). Mouse galectin-3 protein expressed from the 6xHis tag vector is slightly larger at predicted weight $30.4 \mathrm{kDa}$ compared to $29.1 \mathrm{kDa}$ for human galectin-3. Protein concentrations obtained using this method were in the range of 0.1 to $0.3 \mathrm{mg} / \mathrm{ml}$ galectin-3. 
Figure 3.6 - SDS-PAGE analysis of His-tagged recombinant mouse and human galectin-3.

A) FT W1 W2 W3 E1 E2 E3 E4 E5

B)
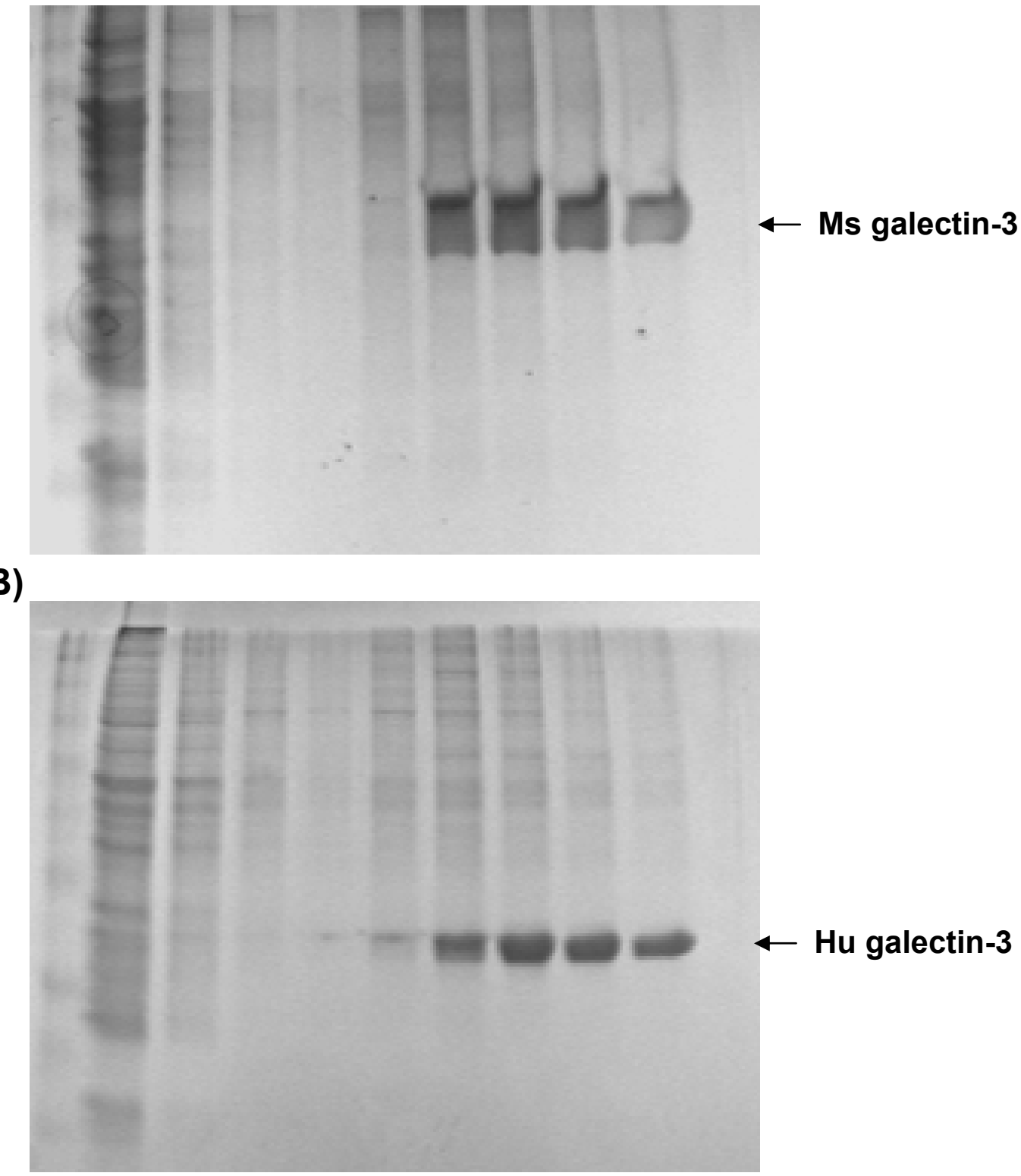

Figure 3.6 (A, B)

His-tagged recombinant galectin-3 was extracted from Sf9 insect cells through binding to Ni-NTA His bind resin. Aliquots of flow through (FT), washes (W1-3) and elutions (E1-5) from mouse galectin-3 (A) and human galectin-3 (B) purifications were analysed using SDS-PAGE. 
Figure 3.7 - Western blot analysis of recombinant mouse and human galectin-3.

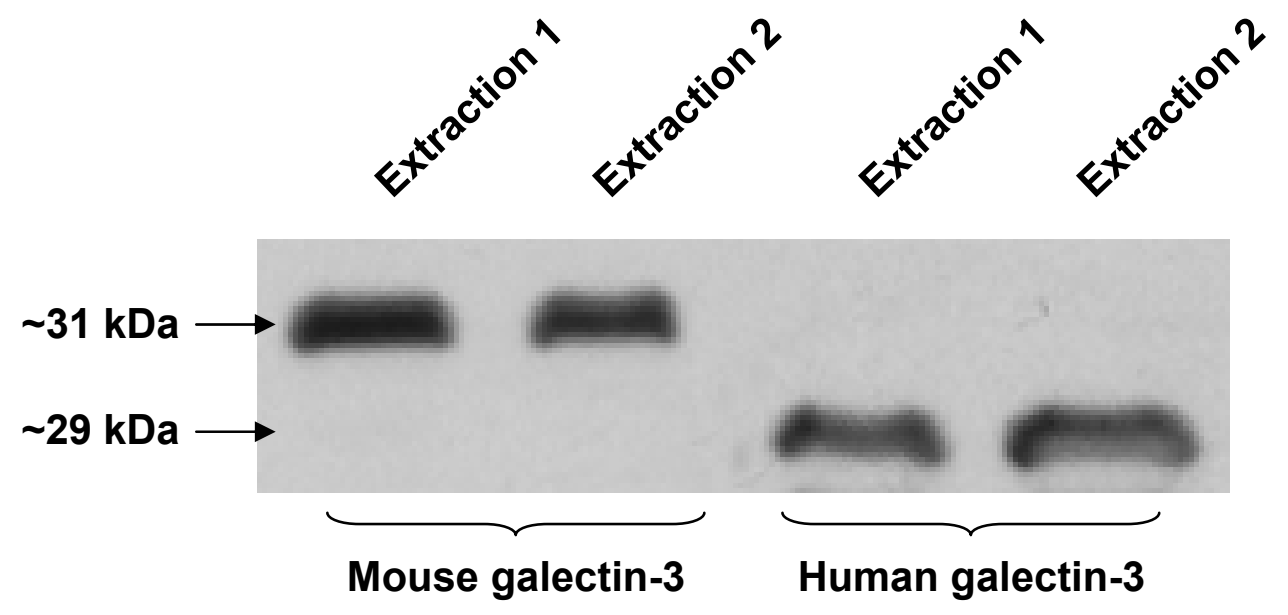

Figure 3.7

Western blot of recombinant mouse and human galectin-3 from two separate purifications. 


\subsection{Discussion}

Recombinant galectin-3 protein concentrations obtained using this method were relatively low and more work needs to be done to optimise this technique. Although yields were low, this technique did produce enough recombinant galectin-3 to use in subsequent experiments. Most importantly the protein produced was pure and will not be contaminated with endotoxin which is possible with protein purified from bacteria. This is particularly true for galectin-3 as it has been shown to bind LPS (Mey et al., 1996). Previous work in the laboratory has shown that attempts to remove endotoxin from bacterially-produced galectin-3 using an endotoxin removal column results in a reduction in the yield of protein recovered.

Insect protein processing pathways may not be equivalent to those of higher eukaryotes - however they produce proteins which are more authentic in glycosylation state and protein folding compared to protein produced using a bacterial expression system. 


\section{Chapter 4}

\section{Galectin-3 plays a critical role in organ fibrosis}

\subsection{Introduction}

Fibrosis, the final common pathway of persistent tissue injury, is a wound healing response to a variety of chronic stimuli. Chronic inflammation with the formation of scar tissue, loss of tissue architecture and organ failure is a characteristic feature of the pathogenesis of many human diseases and represents a major cause of morbidity and mortality worldwide. Currently our therapeutic repertoire is limited to immunosuppression and/or organ transplantation (Neuberger, 2000). The first liver transplant took place in 1963 and there have been vast improvements made since then with one-year survival rates exceeding $80 \%$ (Thalheimer and Capra, 2002). However, donor organs are limited and effective alternative therapies are urgently required.

Until recently fibrosis was thought to be irreversible (Bonis et al., 2001). The extent of clinical and experimental evidence for reversibility of fibrosis is ever increasing. Results from a clinical trial of patients with hepatitis $\mathrm{C}$ showed that $49 \%$ of patients with cirrhosis demonstrated reversal of fibrosis after anti-viral treatment (Poynard et al., 2002). Reversibility of other liver diseases and fibrosis in other organs such as the kidney and heart has also been observed (Elsharkawy et al., 2005). Iredale et al. used the well established carbon tetrachloride $\left(\mathrm{CCl}_{4}\right)$ in vivo model of liver fibrosis to experimentally prove that liver fibrosis can be reversed. Relatively advanced fibrosis in rat liver following injury induced by four weeks of $\mathrm{CCl}_{4}$ injections can be resolved over a short time course (Issa et al., 2004; Iredale et al., 1998). This reversibility has provided another therapeutic target to fight organ fibrosis using anti-fibrotic therapies.

The fibroblast and myofibroblast are key cells in the initiation and perpetuation of organ scarring (Friedman, 2000; Bataller and Brenner, 2005). Classically, quiescent tissue fibroblasts become activated to a contractile, myofibroblast matrix-secreting phenotype. Understanding the molecular 
mechanisms that drive this phenotype switch may allow the development of targeted anti-fibrotic therapies.

Hepatic stellate cells (HSC); originally termed lipocytes, Ito cells or perisinusoidal cells, are the main collagen producing cells of the liver (Bataller and Brenner, 2005). Chronic liver injury results in these cells acquiring fibrogenic properties with increased expression of alpha smooth muscle actin ( $\alpha$-SMA). Following culture on tissue culture plastic, HSCs activate to the myofibroblast phenotype and have been used as a paradigm cell type to study the pathogenesis of tissue fibrosis.

It is well known that type $\beta$ transforming growth factor (TGF- $\beta$ ) is an important cytokine in the production, deposition and contraction of extracellular matrix (ECM) from fibroblasts resulting in excessive scarring and fibrosis. TGF- $\beta$ signalling occurs via the Smad family of transcriptional activators (figure 4.1). TGF$\beta$ receptor I kinase phosphorylates Smad-2 and Smad-3 which then bind Smad-4 and translocate to the nucleus where they induce gene transcription (Heldin et al., 1997; Roberts, 1999). Smad-7 is an inhibitor of Smad signalling and exerts its effects through association with the activated type I receptor thus interfering with Smad-2 and Smad-3 phosphorylation by the receptor (Nakao et al., 1997; Hayashi et al., 1997). Another major intermediate for TGF- $\beta$ signalling are mitogen-activated protein kinases (MAPKs) including extracellular signal-regulated kinase (ERK), cJun N-terminal kinase (JNK) and p38 MAPK, whose signalling pathways can further regulate Smad signalling (Derynck and Zhang, 2003). Studies by Tsukada et al. have demonstrated the importance of both Smad and p38 MAPK signalling in HSC activation and collagen gene expression. They showed that both pathways independently, yet additively, increase $\alpha 1(\mathrm{I})$ collagen gene expression by transcriptional activation and contribute to the expression of $\alpha$-SMA in HSCs. They also demonstrated that p38 MAPK, but not Smad signalling, increased $\alpha 1(\mathrm{I})$ collagen mRNA stability. 
Figure 4.1 - TGF- $\beta /$ Smad signalling pathway.

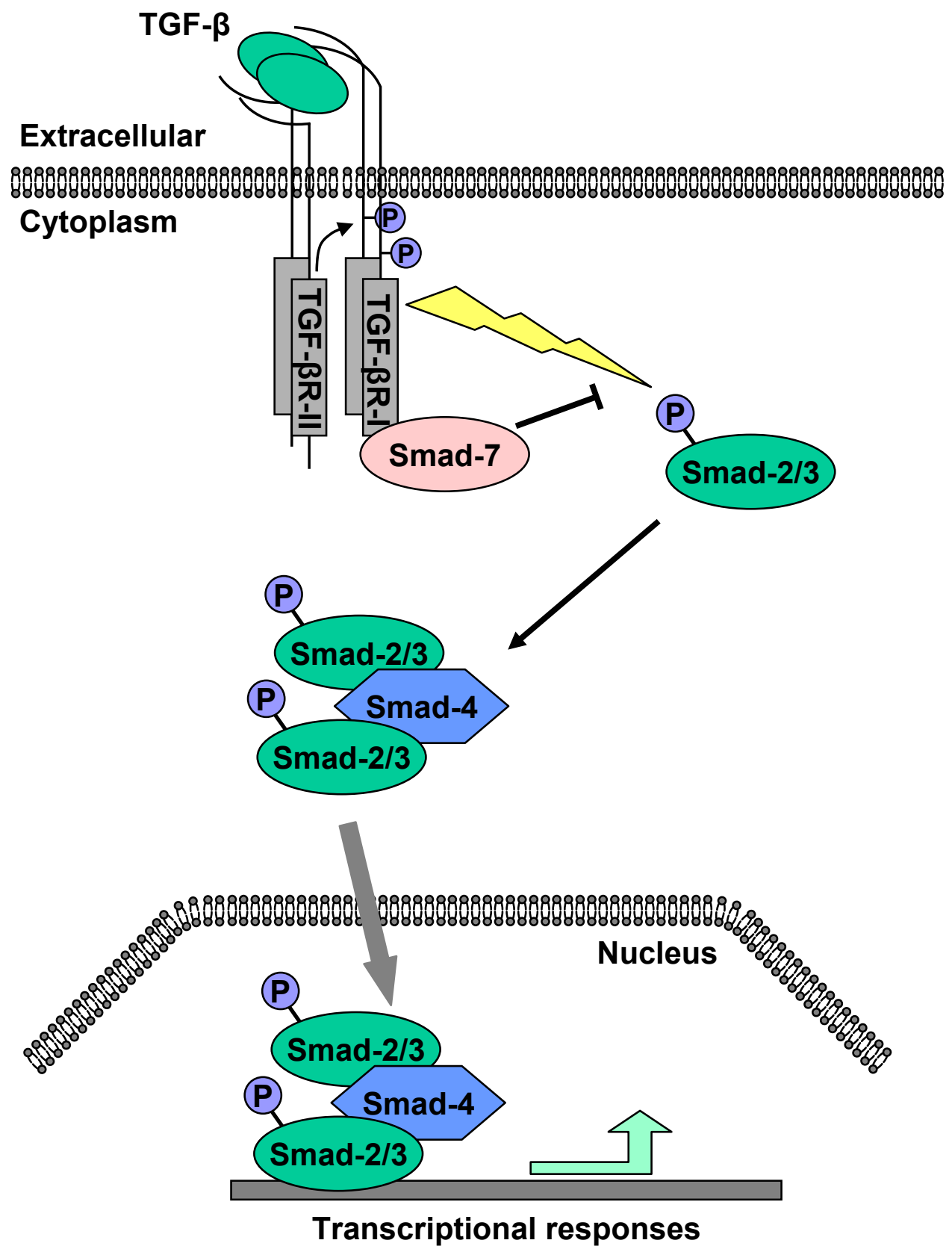

Figure 4.1

TGF- $\beta$ R-II phosphorylates TGF- $\beta$ R-I on a cluster of serine/threonine residues. Activated TGF- $\beta R-I$ propogates the signal downstream by directly phosphorylating Smad-2 and Smad-3. These form complexes with Smad-4 and translocate into the nucleus where they induce gene transcription. Smad-7 inhibits Smad signalling through association with TGF- $\beta$ R-I thus interfering with Smad2/3 phosphorylation. 
Mechanisms of tissue fibrosis also exist that are TGF- $\beta$ independent both in the liver and other organs. Interleukin-13 (IL-13) ${ }^{-/-}$mice infected with Schistosoma mansoni show almost complete abrogation of fibrosis despite continued production of TGF- $\beta$ (Kaviratne et al., 2004). Angiotensin II, which contributes to the progression of vascular fibrosis, has been proven to activate Smad signalling in vascular cells, independently of TGF- $\beta$ (Rodriguez-Vita et al., 2005).

In vitro galectin-3 has been implicated in a variety of biological processes including cell proliferation (Moutsatsos et al., 1987; Inohara et al., 1998), adhesion (Kuwabara and Liu, 1996; Inohara and Raz, 1995; Inohara et al., 1996) and survival (Yang et al., 1996; Akahani et al., 1997). Initial in vivo studies demonstrated that galectin- $3^{-/-}$mice have attenuated peritoneal inflammatory responses to thioglycollate instillation (Colnot et al., 1998b; Hsu et al., 2000), suggesting a role for galectin-3 in the development of acute inflammation. However, the mechanisms that are involved in fibrogenesis are distinct from those involved in inflammation (Strieter and Keane, 2004). Increased galectin-3 expression has been noted in tissue fibrosis (Hsu et al., 1999; Wang et al., 2000; Kasper and Hughes, 1996) and in vitro exogenous galectin3 stimulates myofibroblast proliferation via the MEK1/2-ERK1/2 signalling pathway (Maeda et al., 2003). The relevance of these observations to the mechanistic role of galectin-3 in the pathogenesis of tissue fibrosis in vivo has not been examined.

The role of galectin-3 in the development of fibrosis will be examined using two well characterised models of liver and renal fibrosis. The previously mentioned chronic $\mathrm{CCl}_{4}$ model of liver fibrosis established by Iredale et al. and unilateral ureteric obstruction (UUO) model of renal fibrosis. This model is a very attractive model for studying renal fibrosis as many of the pathophysiological events occur within one week post obstruction (Klahr and Morrissey, 2002). The model of UUO is neutrophil and lymphocyte independent and is characterised by marked tubulointerstitial macrophage infiltration together with progressive scarring (Diamond et al., 1995; Diamond, 1995).

This chapter aims to examine both the phenotypic and mechanistic consequences of an experimental model of chronic hepatic fibrosis using mutant mice lacking the galectin-3 gene. Myofibroblast activation and collagen deposition were investigated and experiments were performed to try and elucidate the pathways 
through which galectin-3 might signal to exert its effects. Phenotypic examination of wild type and galectin- $3^{-/-}$mouse kidneys following UUO-induced renal fibrosis has also been investigated as an additional model of organ fibrosis. The following results were produced in collaboration with Dr Neil Henderson et al. (Henderson et al., 2006).

\subsection{Results}

\subsubsection{Galectin-3 expression is upregulated in human liver fibrosis}

Archival human liver samples obtained from the University of Edinburgh Department of Pathology files were stained for galectin-3 using the DakoCytomation EnVision+ System-HRP (DAB) with the mouse anti-human galectin-3 (clone 9C4) antibody at a 1:100 dilution. Images were acquired using a Leica DM LB light microscope, Leica DC 300 digital camera and Leica IM50 software. In established human liver fibrosis, regardless of aetiology (hepatitis B or C, autoimmune, copper overload, or alcohol-induced), galectin-3 expression was negligible in normal liver compared to a dramatic increase of galectin-3 in the cirrhotic nodules of hepatocytes, particularly at the periphery of the nodules (figure 4.2). Figure 4.2 inset images represent secondary only control staining (labelled polymer-HRP anti-mouse from the DakoCytomation EnVision+ System-HRP kit) of these tissue sections.

\subsubsection{Galectin-3 is temporally and spatially related to fibrosis in a reversible rat model of liver fibrosis}

Galectin-3 and collagen expression was measured in the well established rat model of reversible $\mathrm{CCl}_{4}$-induced liver fibrosis (Issa et al., 2004; Iredale et al., 1998). Rat liver sections were obtained from Professor John Iredale (University of Edinburgh). Sections included those from control rats injected intraperitoneally (i.p.) with olive oil alone, rats chronically injected i.p. with $\mathrm{CCl}_{4}$ twice weekly for 12 weeks in a 1:3 ratio with olive oil and rats injected chronically for 12 weeks then left to recover for 24 weeks post $\mathrm{CCl}_{4}$. 
Figure 4.2 - Galectin-3 expression is upregulated in human liver fibrosis.

\section{Human Liver}

(i) Normal

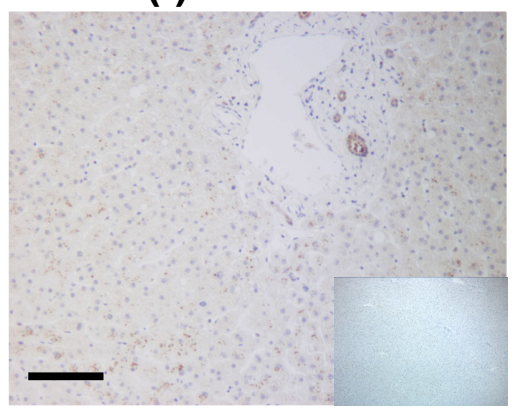

(iii) Hepatitis B

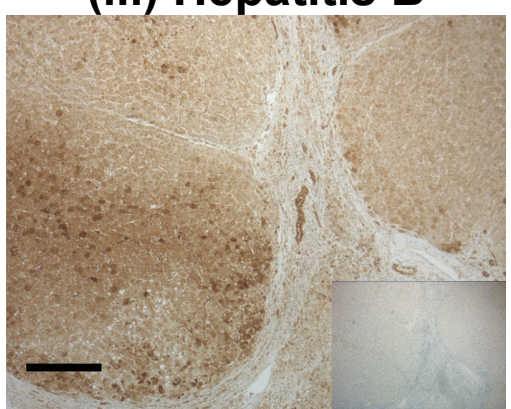

(v) Copper overload

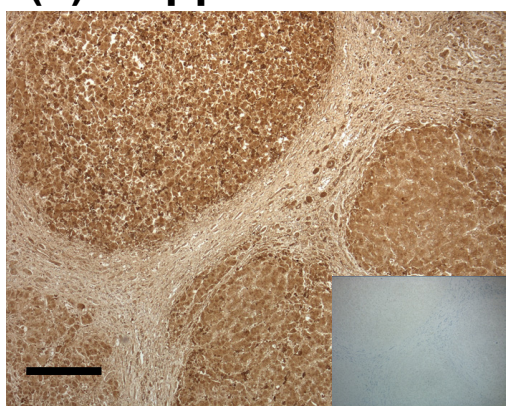

(ii) Hepatitis C

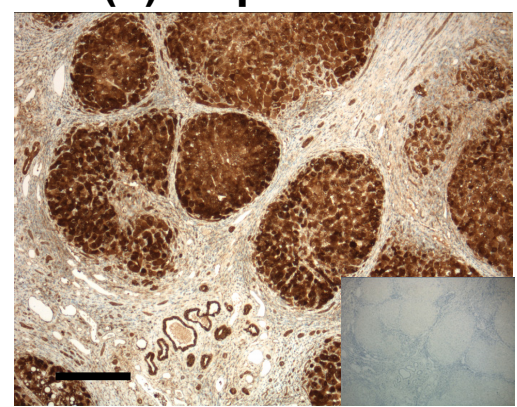

(iv) Autoimmune

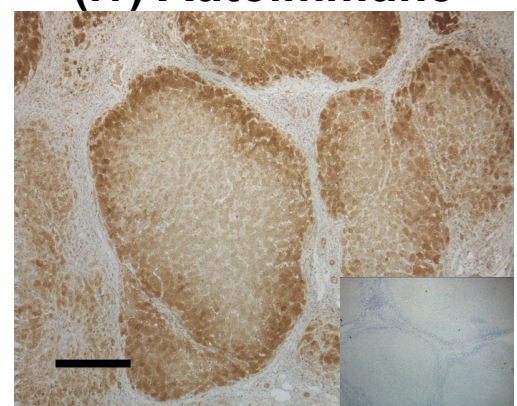

(vi) Alcohol

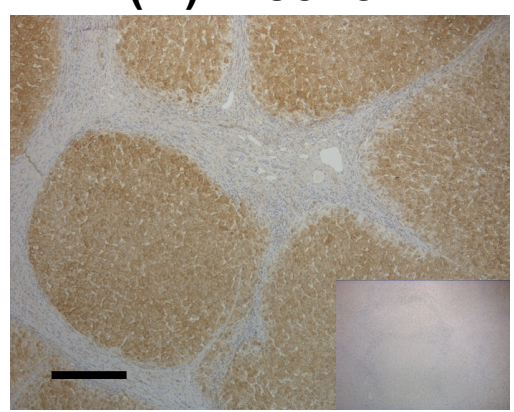

Figure 4.2

Galectin-3 expression in (i) normal human liver and in cirrhosis secondary to (ii) hepatitis $\mathrm{C}$ infection ( $\mathrm{n}=10)$, (iii) hepatitis $\mathrm{B}$ infection $(\mathrm{n}=3)$, (iv) autoimmunity $(\mathrm{n}=3)$, (v) copper overload $(\mathrm{n}=2)$ and (vi) alcohol abuse $(\mathrm{n}=2)$. Inset images demonstrate secondary antibody only control staining for each slide. (Scale bar: $400 \mu \mathrm{m}$.). 
Figure 4.3 - Galectin-3 is temporally and spatially related to fibrosis in a reversible rat model of liver fibrosis.

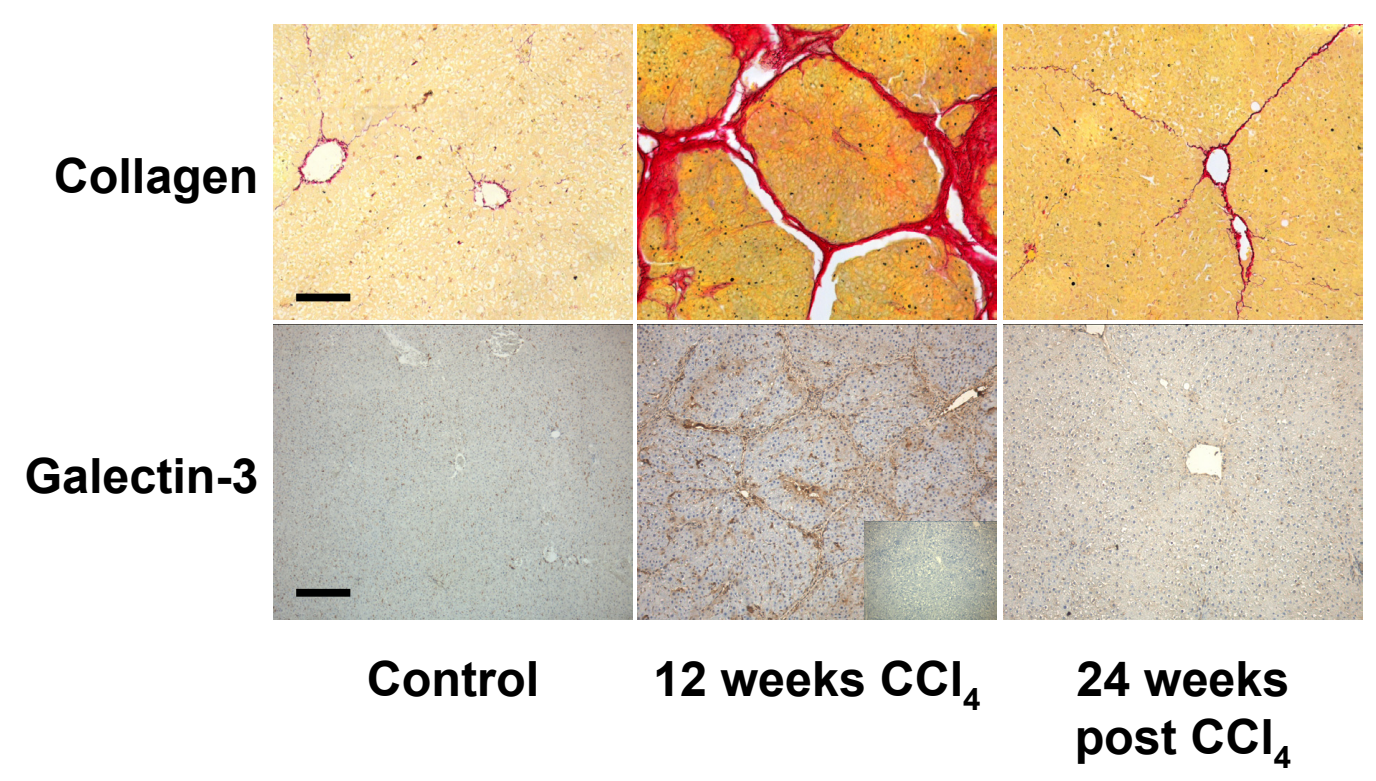

Figure 4.3

Collagen expression stained with PSR (Upper panels, scale bar: $100 \mu \mathrm{m})$ and galectin-3 expression (Lower panels, scale bar: $200 \mu \mathrm{m}$.) in rat liver after 12 weeks of twice-weekly i.p. olive oil vehicle only (Control) (Left), peak fibrosis in rat liver after 12 weeks of twice-weekly i.p. $\mathrm{CCl}_{4}$ (Middle) and resolution, 24 weeks after cessation of $\mathrm{CCl}_{4}$ induced liver injury (Right). Inset image demonstrates secondary antibody only control staining. 
Collagen fibres were stained with picrosirius red (PSR) as a marker of fibrosis (figure 4.3 upper panels) and sections were also stained for galectin-3 using the rabbit anti-rat galectin-3 antibody kindly donated by Professor Fu-Tong Liu (University of California, San Diego) (figure 4.3 lower panels). Figure 4.3 inset images represent secondary only control staining (biotinylated goat anti-rabbit) of this tissue section.

Images were acquired using a Leica DM LB light microscope, Leica DC 300 digital camera and Leica IM50 software. Galectin-3 expression was temporally and spatially associated with fibrosis, minimal in normal rat liver, maximal at peak fibrosis, and was visually absent again at 24 weeks once the liver had recovered from fibrosis. This finding suggested that the development and resolution of fibrosis may be regulated by galectin-3.

\subsubsection{Galectin-3 plays a critical role in liver fibrosis}

Initial data from liver sections obtained from human and rat, suggesting that galectin-3 may be involved in the occurrence of fibrosis, led on to the development of a model system for chronic liver fibrogenesis in mice expressing or deficient in galectin-3. For the chronic fibrosis model mice were injected i.p. for 8 weeks, twice weekly, with olive oil alone (control) or for 8 weeks, twice weekly, with a 1:3 ratio of $\mathrm{CCl}_{4}$ to olive oil, giving a total of 16 injections. Livers were removed, fixed in formaldehyde and paraffin embedded, sectioned and stained for mouse galectin-3, collagen and $\alpha$-SMA as a marker of fibrosis. Images were acquired using a Leica DM LB light microscope, Leica DC 300 digital camera and Leica IM50 software.

Sections were stained for mouse galectin-3 using the anti-mouse Mac-2-FITC antibody (clone $\mathrm{M} 3 / 38$ ) at 1:200 dilution. After chronic $\mathrm{CCl}_{4}$ treatment, increased galectin-3 expression was observed in the periportal areas and dense staining was noted at the periphery of the inflamed damaged areas. In liver from wild type control animals, galectin-3 was only expressed in bile duct epithelia and Kupffer cells in the liver (figure 4.4A). Figure 4.4A inset image represents secondary only control staining (rabbit anti-FITC) of this tissue section. Real-time RT-PCR for galectin-3 was performed on RNA extracted from whole liver and galectin-3 protein expression was measured by Western blotting. Both galectin-3 mRNA levels and protein 
expression were increased with the development of hepatic fibrosis in the $\mathrm{CCl}_{4-}$ treated animals compared with control (figure 4.4D and E).

Collagen fibres on liver sections from wild type and galectin- $3^{-/-}$mice treated chronically with $\mathrm{CCl}_{4}$ were stained with PSR as a marker of fibrosis. Staining of wild type livers demonstrated the presence of hepatic collagen with the same distribution as galectin-3 (figure 4.4B). Collagen was present in the periportal areas and areas of bridging fibrosis in wild type livers compared to significantly less collagen deposition in the galectin- $3^{-/-}$livers. Staining was quantified using Openlab software for digital image analysis (figure 4.4F). Details of the quantitation are given in the Materials and Methods Chapter. Procollagen (I) mRNA expression was analysed by real-time RT-PCR and was significantly decreased in the livers from galectin- $3^{-/-}$compared to wild type mice after chronic $\mathrm{CCl}_{4}$ treatment (figure $4.4 \mathrm{G}$ ). This result suggested that galectin-3 is associated with hepatic collagen deposition during liver injury. It was therefore important to examine the mechanism underlying this important observation.

Following liver injury, HSCs activate to a proliferative, fibrogenic and contractile myofibroblast with increased expression of $\alpha$-SMA, a widely accepted marker of myofibroblast activation (Friedman, 2000; Bataller and Brenner, 2005) in vitro and in vivo. After 8 weeks of $\mathrm{CCl}_{4}$ treatment, liver sections were stained for $\alpha$ SMA using the anti- $\alpha$-SMA-FITC antibody (clone 1A4) at 1:15,000 dilution. $\alpha$ SMA expression was significantly increased in wild type compared to galectin- $3^{-/-}$ liver sections with the same spatial and temporal distribution as galectin-3 and collagen expression (figure 4.4C). Figure 4.4C inset image represents secondary only control staining (rabbit anti-FITC) of this tissue section. Staining was quantified using digital image analysis (figure 4.4H). $\alpha$-SMA mRNA expression was analysed by real-time RT-PCR and was significantly decreased in the livers from galectin- $3^{-/-}$compared to wild type mice after chronic $\mathrm{CCl}_{4}$ treatment (figure 4.4I). This decrease in $\alpha$-SMA mRNA expression was paralleled by a decrease in hepatic $\alpha$-SMA protein expression assessed by Western blot analysis of whole liver tissue (figure 4.4I inset). These data indicate that galectin-3 mediated activation of hepatic stellate cells in vivo is a central mechanism underlying hepatic fibrosis. 
Figure 4.4 - Galectin-3 plays a critical role in liver fibrosis.

A)

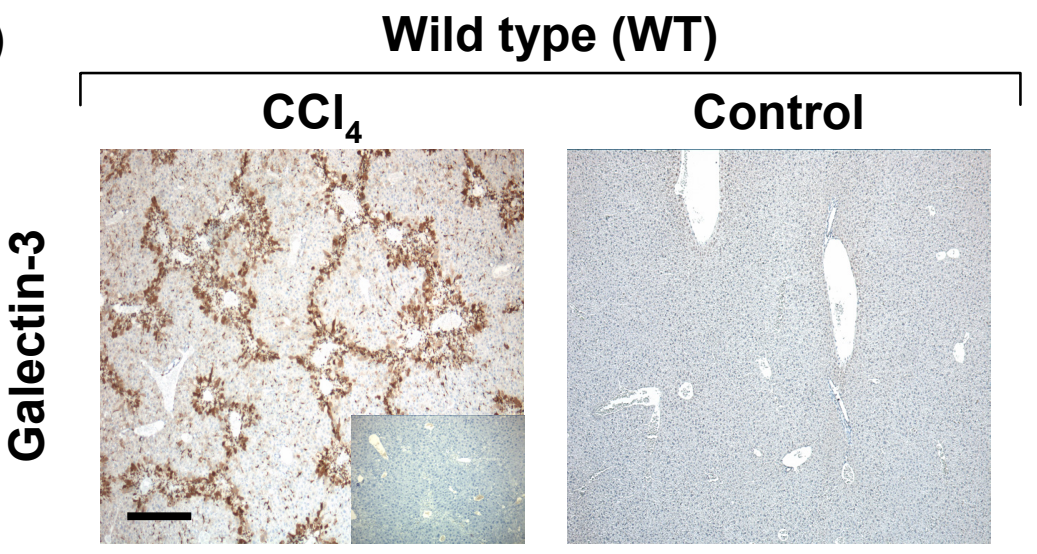

B)
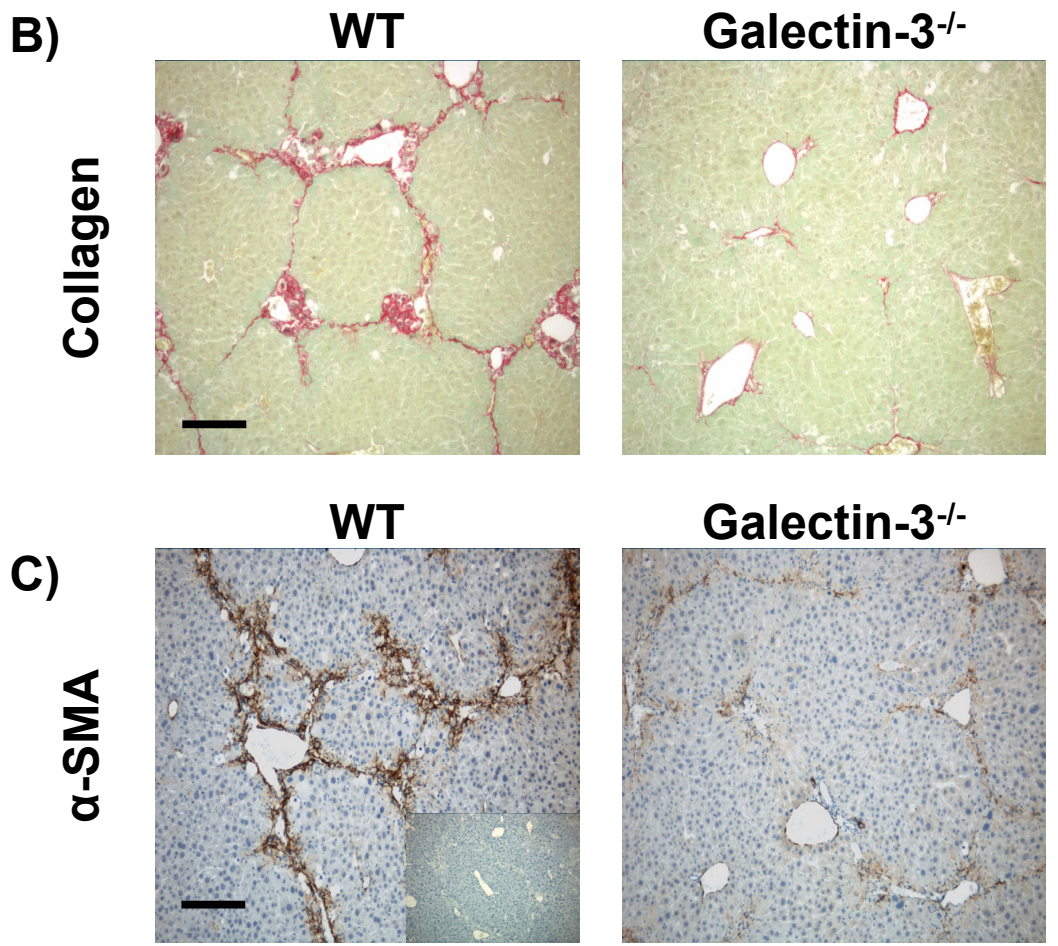

Figure 4.4 (A-C)

Mice were treated with olive oil (control) or $\mathrm{CCl}_{4}$ i.p. twice weekly for 8 weeks ( $\mathrm{n}=6$ mice in each group). (A) Galectin-3 expression in control (Right) and after chronic $\mathrm{CCl}_{4}$ treatment (Left) in WT mouse liver. (Scale bar: $400 \mu \mathrm{m}$ ). Inset image demonstrates secondary antibody only control staining. (B) Collagen staining with PSR of liver tissue after chronic $\mathrm{CCl}_{4}$ treatment of WT and galectin$3^{-/-}$mice. (Scale bar: $200 \mu \mathrm{m}$ ). (C) $\alpha$-SMA staining of liver tissue after chronic $\mathrm{CCl}_{4}$ treatment of WT and galectin- $3^{-/-}$mice. (Scale bar: $200 \mu \mathrm{m}$ ). Inset image demonstrates secondary antibody only control staining. 


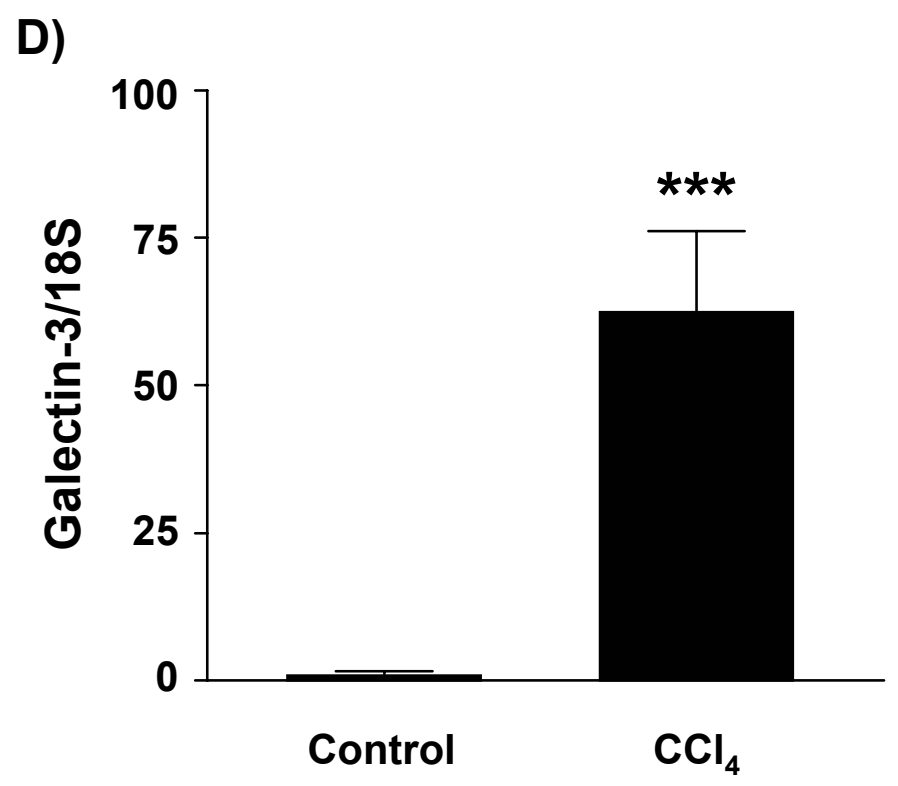

E)

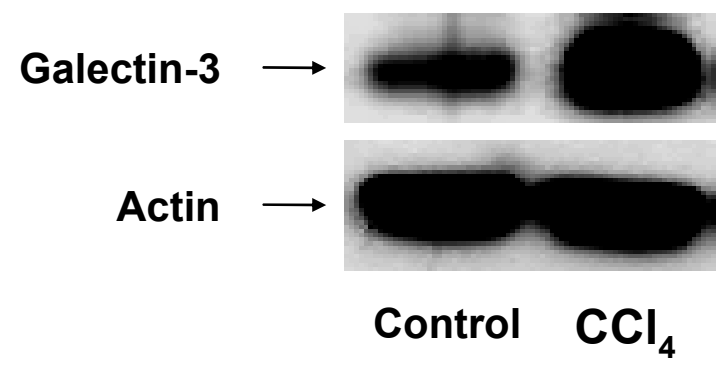

Figure 4.4 (D, E)

(D) Real-time RT-PCR quantitation of galectin-3 expression (normalised to $18 \mathrm{~S}$ ) in total RNA extracts from livers from control (olive oil vehicle) and chronic $\mathrm{CCl}_{4}$ treated WT mice. ${ }^{* * *}, \mathrm{P}<0.0001$ compared to control. Real-time RT-PCR results are expressed as fold change relative to control. (E) Representative galectin-3 and actin Western blots of whole liver from control and $\mathrm{CCl}_{4}$ treated WT mice. 


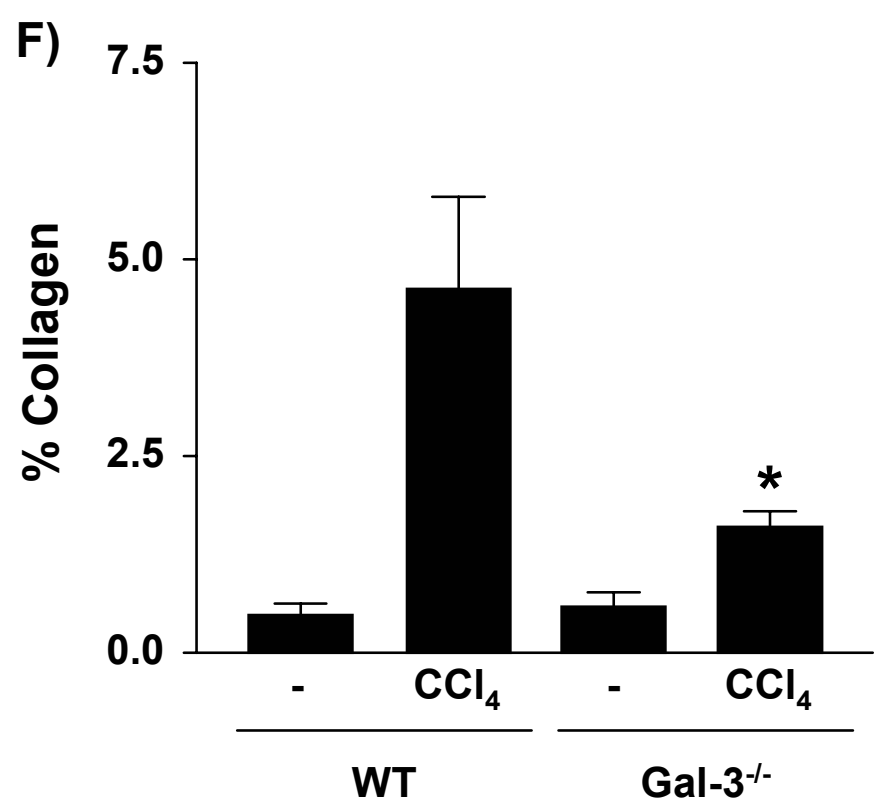

G)

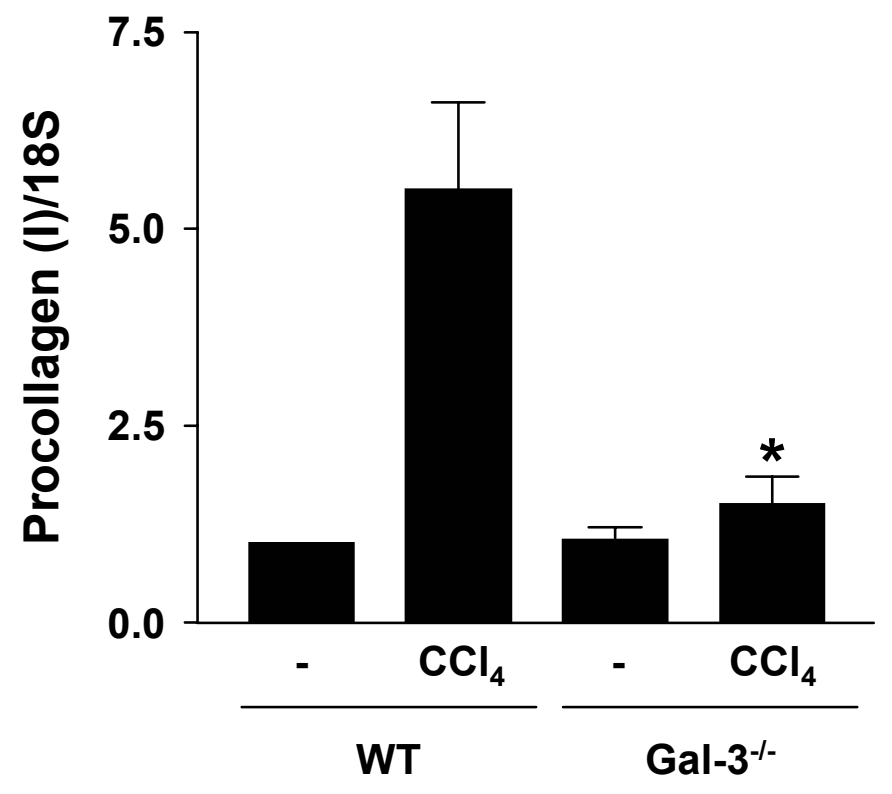

Figure 4.4 (F, G)

(F) Digital image analysis quantitation of collagen staining. *, $\mathrm{P}<0.05$ compared to $\mathrm{WT} \mathrm{CCl}_{4}$. (G) Real-time RT-PCR quantitation of procollagen (I) expression (normalised to $18 \mathrm{~S}$ ) in total RNA extracts from livers from control (olive oil vehicle) and chronic $\mathrm{CCl}_{4}$ treated $\mathrm{WT}$ and galectin- $3^{-/}$mice. ${ }^{*}, \mathrm{P}<0.05$ compared to $\mathrm{WT} \mathrm{CCl}_{4}$. Real-time RT-PCR results are expressed as fold change relative to WT control. 

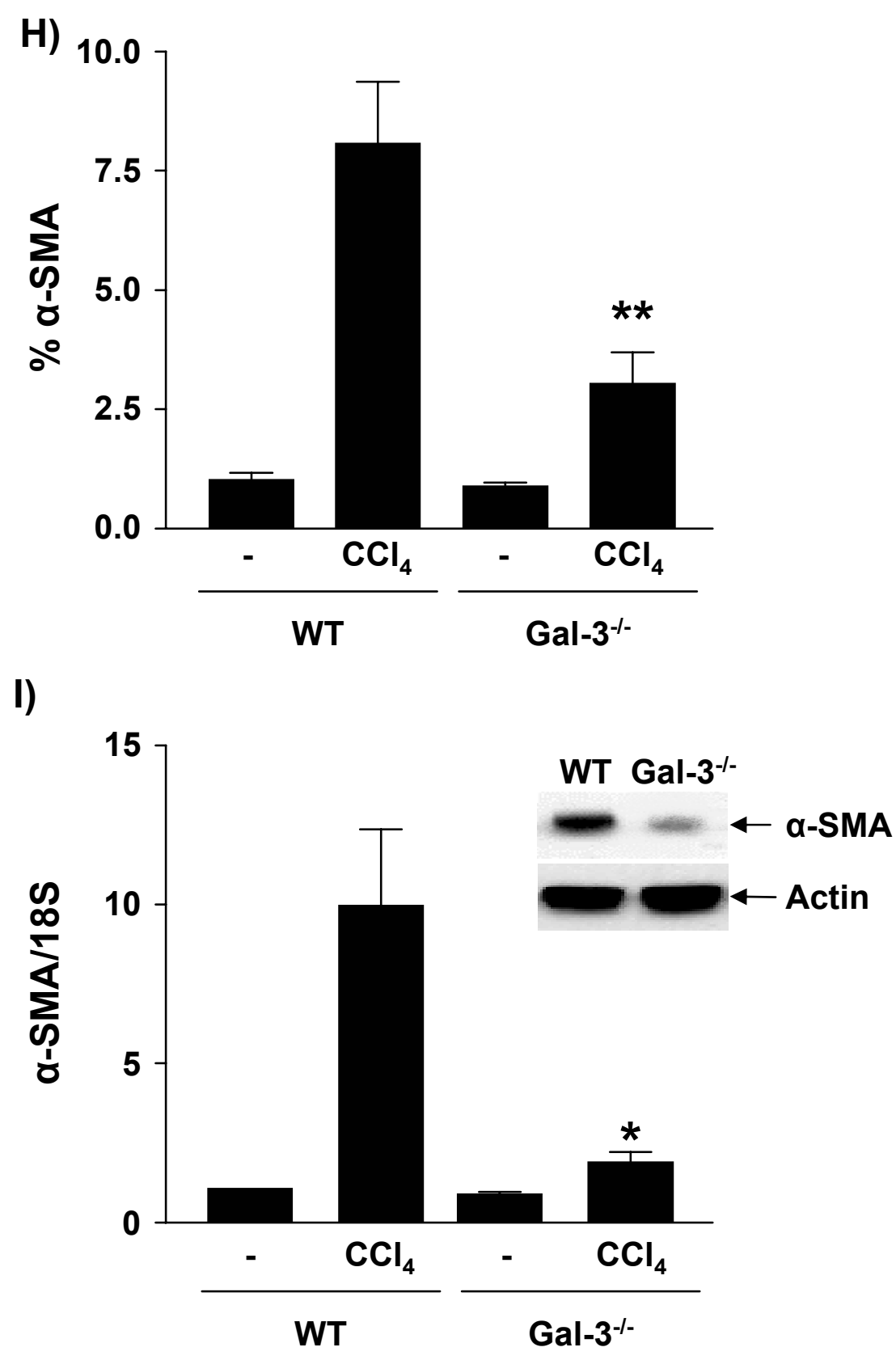

Figure 4.4 (H, I)

(H) Digital image analysis quantitation of $\alpha$-SMA staining. ${ }^{* *}, \mathrm{P}<0.01$ compared to $\mathrm{WT} \mathrm{CCl}_{4}$. (I) Real-time RT-PCR quantitation of $\alpha$-SMA expression (normalised to $18 \mathrm{~S}$ ) in total RNA extracts from livers from control (olive oil vehicle) and chronic $\mathrm{CCl}_{4}$ treated $\mathrm{WT}$ and galectin- $3^{-/}$mice. ${ }^{*}, \mathrm{P}<0.05$ compared to $\mathrm{WT} \mathrm{CCl}_{4}$. Real-time RT-PCR results are expressed as fold change relative to WT control. (I Inset) Representative Western blots of $\alpha$-SMA and actin expression in whole liver from chronic $\mathrm{CCl}_{4}$-treated mice. 


\subsubsection{Myofibroblast activation is galectin-3 dependent}

HSC activation to a myofibroblast phenotype is a critical event in ECM deposition and cirrhosis (Friedman, 2000; Bataller and Brenner, 2005). Mouse HSCs were isolated from wild type and galectin- $3^{-/-}$livers as described in Materials and Methods. HSCs were plated at $1 \times 10^{6}$ cells/well in a 6-well plate, in the presence of $16 \%$ FCS and lysed at 0, 2, 7 and 10 days. Galectin-3 protein expression was upregulated during myofibroblast activation in vitro on tissue culture plastic in primary mouse HSCs (figure 4.5A). This well established in vitro method of HSC activation closely models in vivo myofibroblast activation (Issa et al., 2004; Iredale et al., 1998) and has been used extensively to model and examine changes that take place during the phenotype switch of fibroblasts to ECM-secreting contractile myofibroblasts. After 7 days in vitro culture, $\alpha$-SMA protein expression was significantly less in galectin- $3^{-/-}$HSCs compared with wild type HSCs (figure 4.5B). Addition of exogenous recombinant murine galectin-3 to galectin- $3^{-/-}$HSCs in vitro for 48 hours reversed the galectin- $3^{-/ 2}$ phenotype resulting in increased $\alpha$-SMA expression (figure 4.5C). Rescue of the pro-fibrotic phenotype by exogenous galectin-3 was confirmed with real-time RT-PCR, which demonstrated upregulation of procollagen (I) expression (figure 4.5D). When plated on tissue culture plastic in vitro wild type primary murine $\mathrm{HSCs}(\mathrm{O})$ proliferate faster than galectin- $3^{-/-} \mathrm{HSCs}$ ( $\square$ ) as judged by 3-(4,5-dimethylthiazol-2yl)-2,5-diphenyl tetrazolium bromide (MTT) assay. This defect in proliferation of the galectin- $3^{-/-}$HSCs could be restored

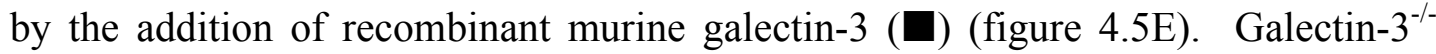
HSCs were seeded in immunofluorescence chambers at 50,000 cells per well in complete media and cultured for 4 days. HSCs were incubated $30 \mu \mathrm{g} / \mathrm{ml} \mathrm{recombinant}$ mouse galectin-3 for 10,30 or 60 minutes and immunofluorescence for galectin-3 using 1:20 anti-galectin-3 (clone A3A12) antibody, was performed. Figure 4.5F shows that galectin-3 is rapidly internalised (within 10 minutes) when added to galectin- $3^{-/}$HSCs and very little galectin-3 accumulates at the membrane. 
Figure 4.5 - Myofibroblast activation is galectin-3 dependent.

A)

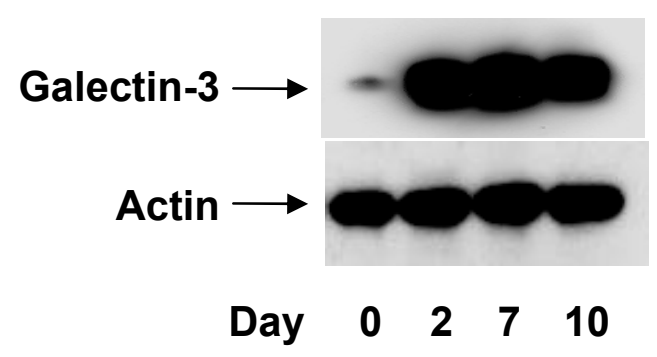

B)

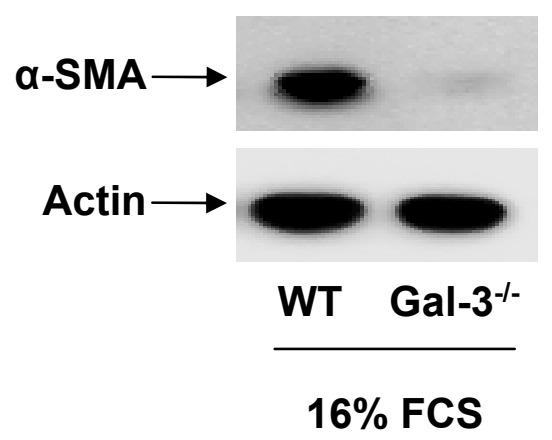

C)

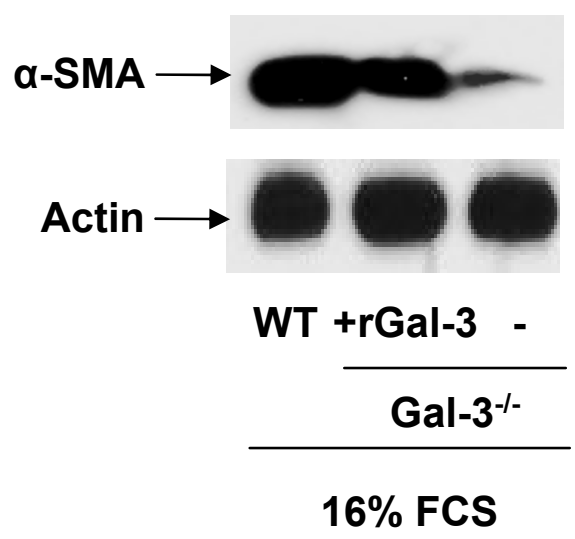

Figure 4.5 (A-C)

(A) Western blot analysis of galectin-3 expression in primary mouse HSCs during transition from the quiescent to the activated phenotype on tissue culture plastic over 10 days in culture. (B) Western blot analysis of $\alpha$-SMA and actin expression in WT and galectin-3 $3^{-/}$primary mouse HSCs cultured on tissue culture plastic for 7 days in $16 \%$ FCS. (C) Western blot analysis of $\alpha$-SMA in WT and galectin- $3^{-1}$ primary mouse HSCs (day 4) after a $48 \mathrm{~h}$ treatment of recombinant mouse galectin-3 $(30 \mu \mathrm{g} / \mathrm{ml})$ to galectin- $3^{-/} \mathrm{HSCs}$ in $16 \% \mathrm{FCS}$. 
D)

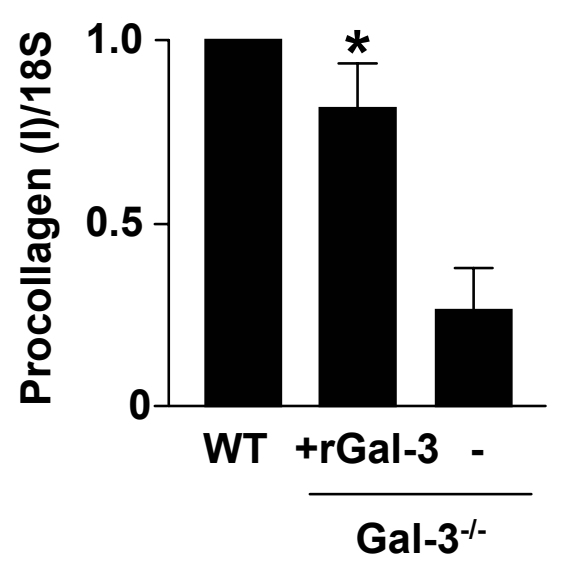

$16 \%$ FCS
E)

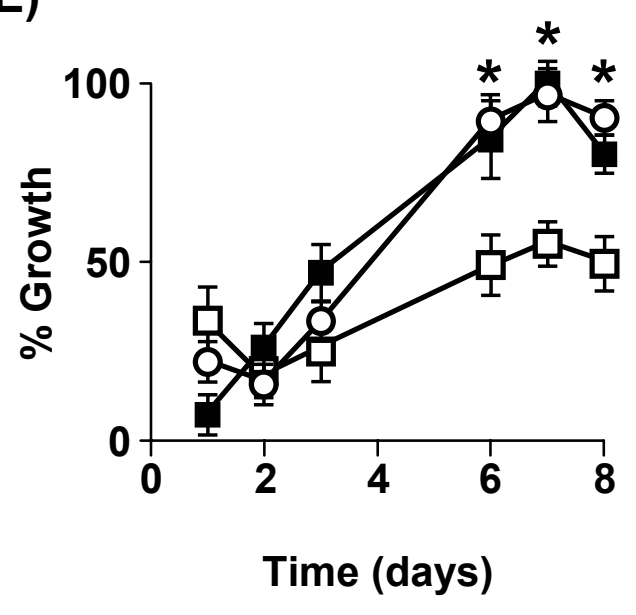

F)

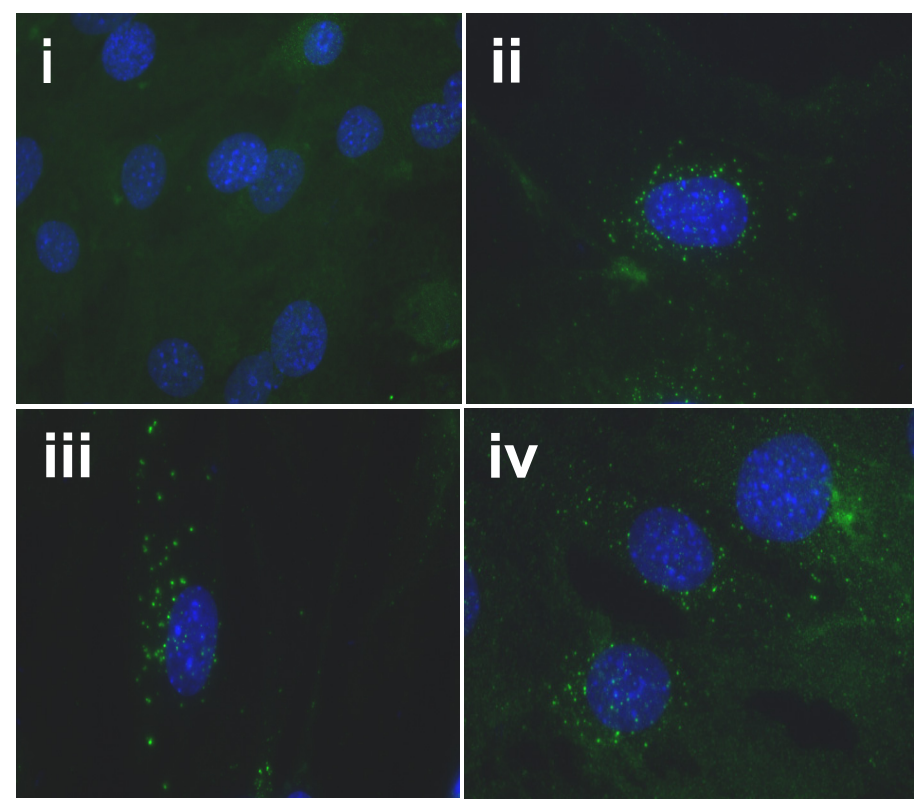

Figure 4.5 (D-F)

(D) Real-time RT-PCR quantitation of procollagen (I) expression (normalised to 18S) in WT and galectin- $3^{-/}$primary mouse HSCs (day 4 ) after $48 \mathrm{~h}$ treatment of recombinant mouse galectin-3 $(30 \mu \mathrm{g} / \mathrm{ml})$ in $16 \%$ FCS $(\mathrm{n}=3) *, \mathrm{P}<0.05$ compared to untreated galectin- $3^{-/-}$HSCs. (E) Cell growth of WT (O), galectin-3-

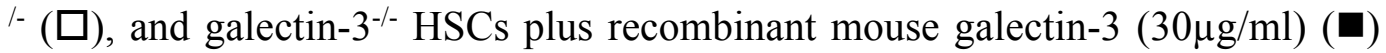
measured by MTT assay $(\mathrm{n}=3) *, \mathrm{P}<0.05$ compared to untreated galectin- $3^{-/-}$ HSCs. $\mathrm{P}=0.09$ (ns), 0.20 (ns) and 0.17 (ns) for days 1,2 and 3 respectively. (F) Internalisation of recombinant mouse galectin-3 by galectin- $3^{-/-}$primary mouse HSCs. Cells were stained with DAPI (blue) and galectin-3 antibody (green). (i) Untreated, (ii) $10 \mathrm{~min}$, (iii) $30 \mathrm{~min}$, (iv) $60 \mathrm{~min}$ following addition of $30 \mu \mathrm{g} / \mathrm{ml}$ recombinant mouse galectin-3. 
A rationally designed small interfering RNA (siRNA) pool targeting galectin3 mRNA was used to inhibit galectin-3 expression in wild type primary murine HSCs. All siRNA was purchased from the Dharmacon siGENOME Collection of pre-designed siRNA reagents. siGENOME siRNA reagents are designed using Dharmacon's SMARTselection siRNA design algorithm to reduce off-target effects and decrease false positives.

HSCs were isolated, activated on tissue culture plastic for 7 days and treated with PBS, liposome formulated non-targeted control duplex or 250nM liposome formulated galectin-3 siRNA duplex 1-4 using Oligofectamine. All four duplexes cause over 95\% knockdown of galectin-3 protein expression 96 hours posttransfection with duplexes 1 and 2 causing the greatest knockdown (figure 4.6A). Subsequent experiments were carried out using mouse galectin-3 siRNA duplex 1 . 96 hours post transfection with galectin-3 siRNA, RNA was extracted using the Qiagen RNeasy Mini Kit, reverse transcribed to complementary DNA (cDNA) and processed using quantitative real-time RT-PCR. Galectin-3 siRNA treatment reduced galectin-3 mRNA expression 10-fold as compared with non-targetting siRNA or mock transfection (figure 4.6B). siRNA knockdown of galectin-3 in HSCs did not affect cell viability assessed by trypan blue exclusion. This marked inhibition of galectin-3 expression resulted in a significant reduction in both $\alpha$-SMA and procollagen (I) mRNA expression assessed by real-time RT-PCR (figure 4.6C and D respectively). Western blot analysis confirmed almost complete inhibition of galectin- 3 expression and a reduction in $\alpha$-SMA protein expression after targeted siRNA treatment relative to control treatment (figure 4.6E). This data supports the premise that galectin-3 expression is required for HSC differentiation into a myofibroblast phenotype. 
Figure 4.6 - Galectin-3 siRNA inhibits myofibroblast activation and procollagen (I) expression in HSCs.

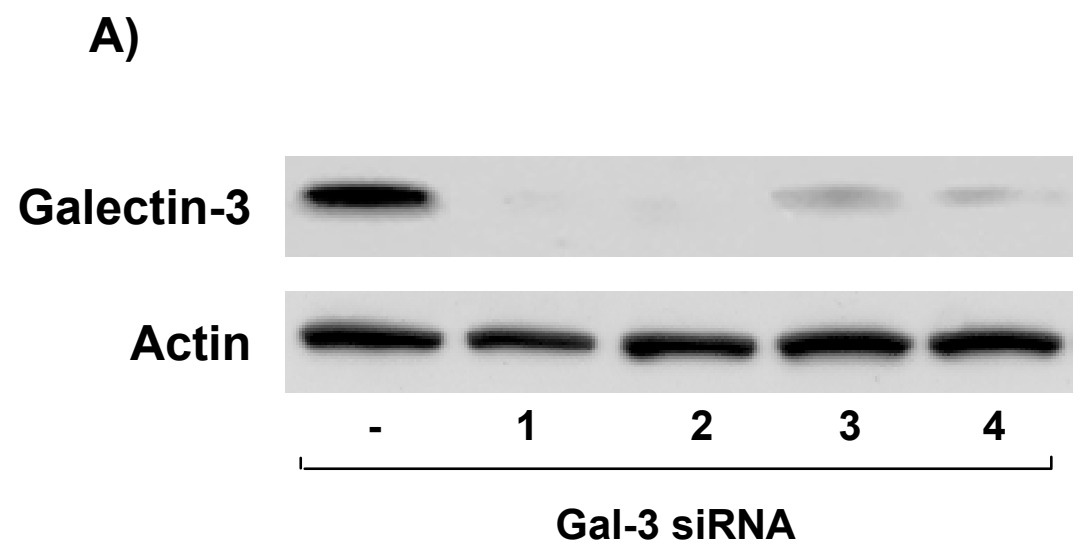

Figure 4.6 (A)

(A) HSCs were transfected with $250 \mathrm{nM}$ galectin-3 siRNA duplexes (1-4 as indicated) as described in Materials and Methods. 96h post-transfection cell lysates were resolved on 12\% SDS-PAGE gels. Blots were probed for galectin-3 and actin expression. 
B)

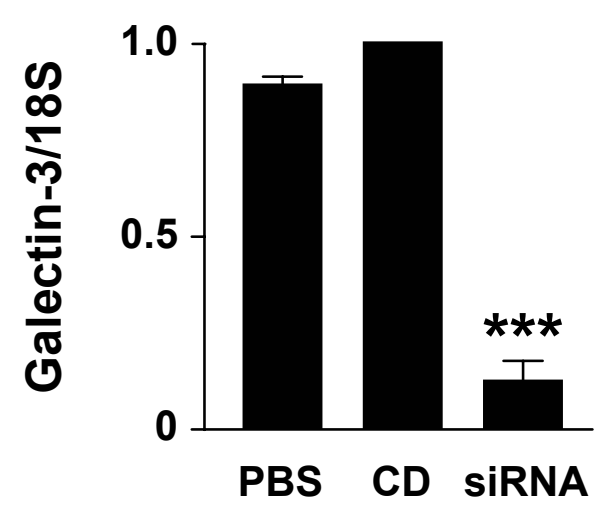

D)

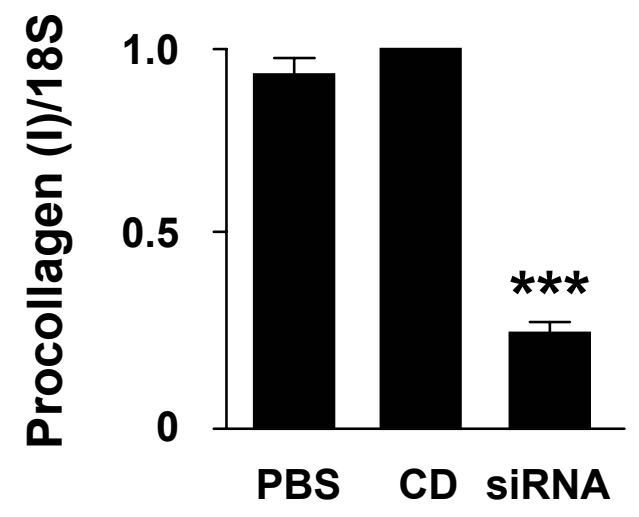

C)

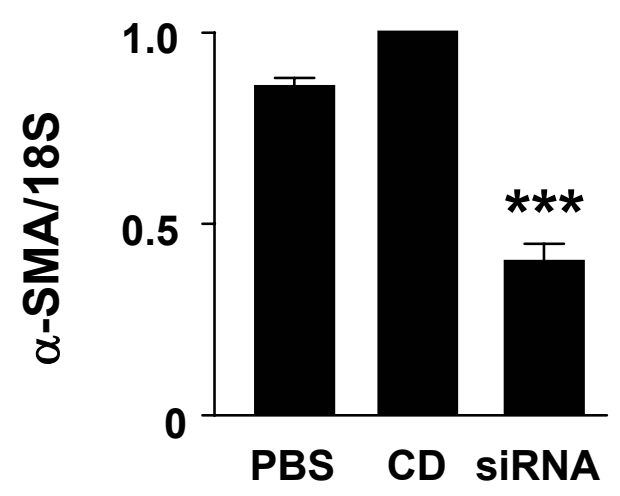

E)

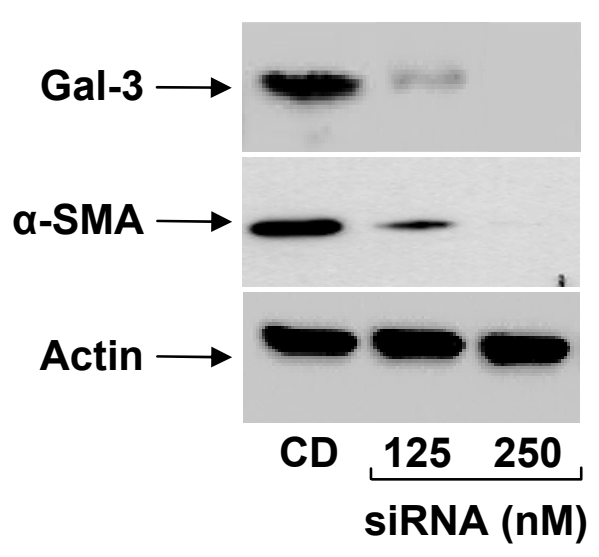

Figure 4.6 (B-E)

Real-time RT-PCR quantitation of (B) galectin-3, (C) $\alpha$-SMA, and (D) procollagen (I) expression (normalised to 18S) in primary mouse HSCs after $96 \mathrm{~h}$ treatment with PBS, control duplex (CD) or $250 \mathrm{nM}$ mouse galectin-3 siRNA duplex $1(\mathrm{n}=3)$. ***, $\mathrm{P}<0.0001$ compared to PBS. Real-time RT-PCR results are expressed as fold change relative to CD. (E) Western blot analysis of galectin-3, $\alpha$-SMA and actin expression in primary mouse HSCs $96 \mathrm{~h}$ after transfection with $\mathrm{CD}$ or $125 \mathrm{nM}$ or $250 \mathrm{nM}$ mouse galectin-3 siRNA duplex 1 . 


\subsubsection{Disruption of the galectin-3 gene does not affect TGF- $\beta$ expression and Smad signalling}

TGF- $\beta$ is a major profibrogenic cytokine involved in the pathogenesis of fibrosis in many different organ systems (Leask and Abraham, 2004; Uemura et al., 2005; Qi et al., 1999). However, mechanisms of tissue fibrosis also exist that are TGF- $\beta$ independent both in the liver and other organs (Kaviratne et al., 2004; Rodriguez-Vita et al., 2005).

The tissue expression of TGF- $\beta$ mRNA was markedly elevated as judged by quantitative real-time RT-PCR in whole liver samples following chronic $\mathrm{CCl}_{4}$ liver injury compared with control. However, there was no significant difference in hepatic TGF- $\beta$ mRNA expression between wild type and galectin- $3^{-/-}$mice in this model of liver fibrosis (figure 4.7A). A mouse TGF- $\beta$ ELISA (R\&D) was performed to determine the concentration of TGF- $\beta$ in supernatants recovered from day 4 wild type and galectin- $3^{-/-}$HSCs cultured for 24 hours in serum-free media (figure 4.7B). This assay demonstrated no difference in the levels of TGF- $\beta$ expression from wild type and galectin- $3^{-/-}$HSCs in tissue culture. Thus disruption of the galectin-3 gene blocks fibrosis despite similar expression levels of TGF- $\beta$. In the presence of TGF- $\beta$ ligand, the receptor-activated Smad family of transcriptional activators, Smad-2 and 3 , are phosphorylated directly by the TGF- $\beta$ receptor I kinase (Heldin et al., 1997; Roberts, 1999). Smad-2 and Smad-3 activation in wild type and galectin-3 ${ }^{-/-}$HSCs treated with TGF- $\beta$ were analysed by Western blotting for pSmad- 2 and pSmad-3 using either the polyclonal anti-pSmad $2 / 3$ or anti-pSmad-3 antibody respectively. $1 \times 10^{7}$ HSCs were seeded onto tissue culture plastic for 4 days, quiesced in serumfree media for 24 hours and incubated with increasing concentrations of TGF- $\beta$ for 60 minutes. TGF- $\beta$ stimulated a similar increase in Smad-2 and Smad-3 phosphorylation in wild type and galectin- $3^{-/-}$HSCs (figure 4.7C) indicating that the pro-fibrotic responses were not due to galectin- 3 signalling via the TGF- $\beta / \mathrm{Smad}$ pathway. 
Figure 4.7 - Galectin-3 regulates myofibroblast activation and hepatic fibrosis despite similar levels of TGF- $\beta$ expression and signalling.

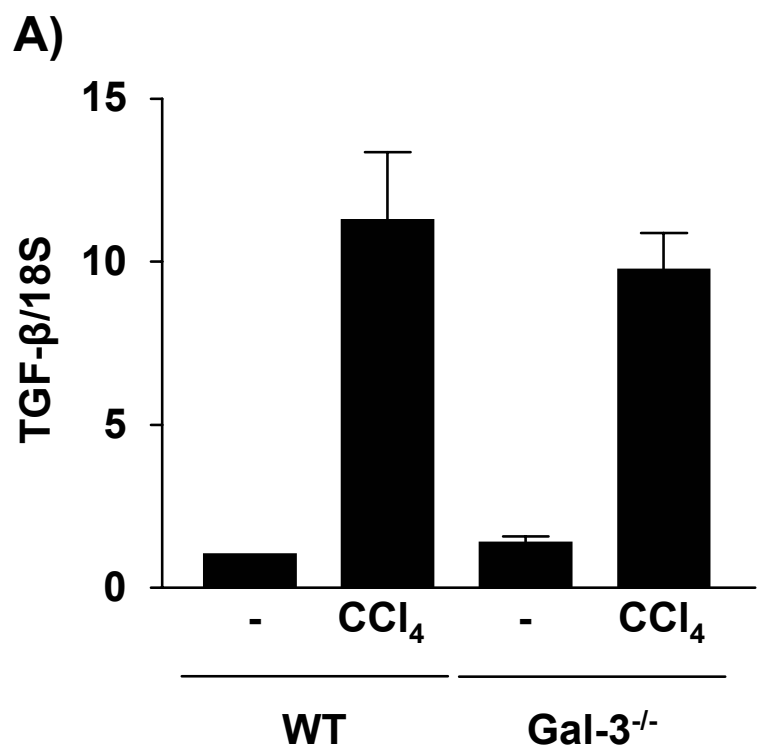

B)

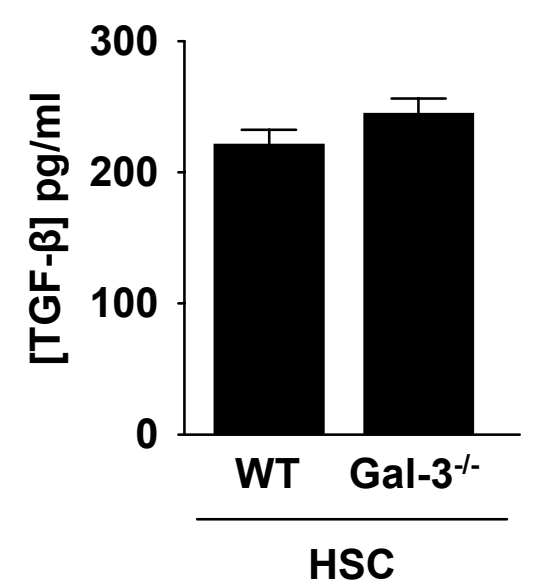

Figure 4.7 (A,B)

(A) Real-time RT-PCR quantitation of TGF- $\beta$ expression (normalised to $18 \mathrm{~S}$ ) in total RNA extracts from livers from control (olive oil vehicle) and chronic (8 weeks) $\mathrm{CCl}_{4}$-treated WT and galectin- $3^{-/-}$mice ( $\mathrm{n}=6$ mice in each group). Realtime RT-PCR results are expressed as fold change relative to WT control. P = 0.37 (ns). (B) TGF- $\beta$ concentration measured by ELISA in supernatants recovered from day $4 \mathrm{WT}$ and galectin- $3^{-/-}$HSCs cultured for $24 \mathrm{~h}$ in serum-free media $(\mathrm{n}=3)$. $\mathrm{P}=0.08(\mathrm{~ns})$. 


\section{C)}
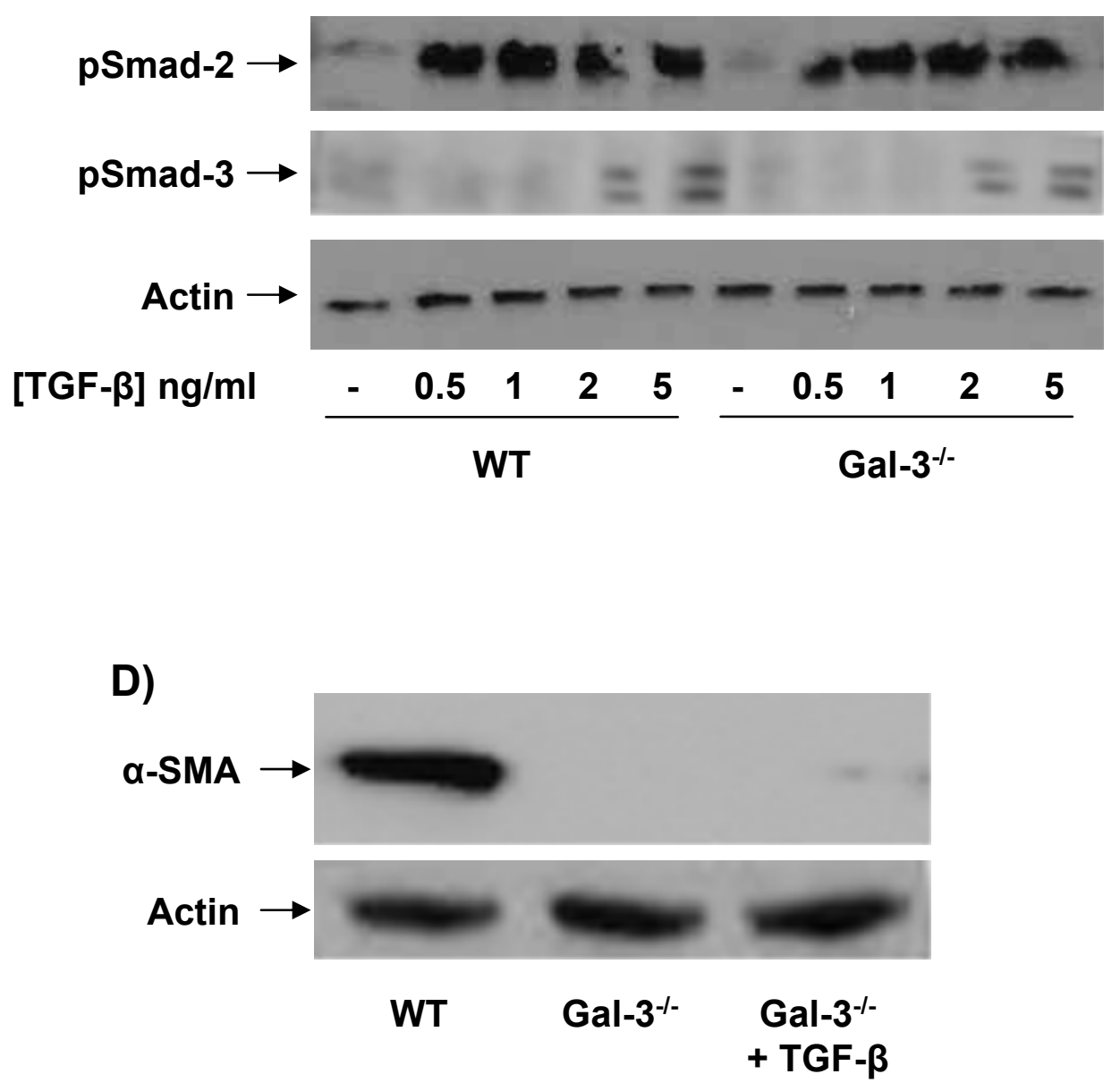

Figure 4.7 (C, D)

(C) HSCs (day 4) were quiesced in serum-free media for $24 \mathrm{~h}$ and stimulated with increasing concentrations of TGF- $\beta$ for $60 \mathrm{~min}$. Lysates were Western blotted for phosphorylated Smad-2 (pSmad-2) and phosphorylated Smad-3 (pSmad-3) as a measure of Smad activation. (D) Western blot analysis of $\alpha$-SMA expression in HSCs (day 4) quiesced in serum-free media for $24 \mathrm{~h}$ and stimulated with $5 \mathrm{ng} / \mathrm{ml}$ TGF- $\beta$ for $48 \mathrm{~h}$. Representative blots of 3 independent experiments. 

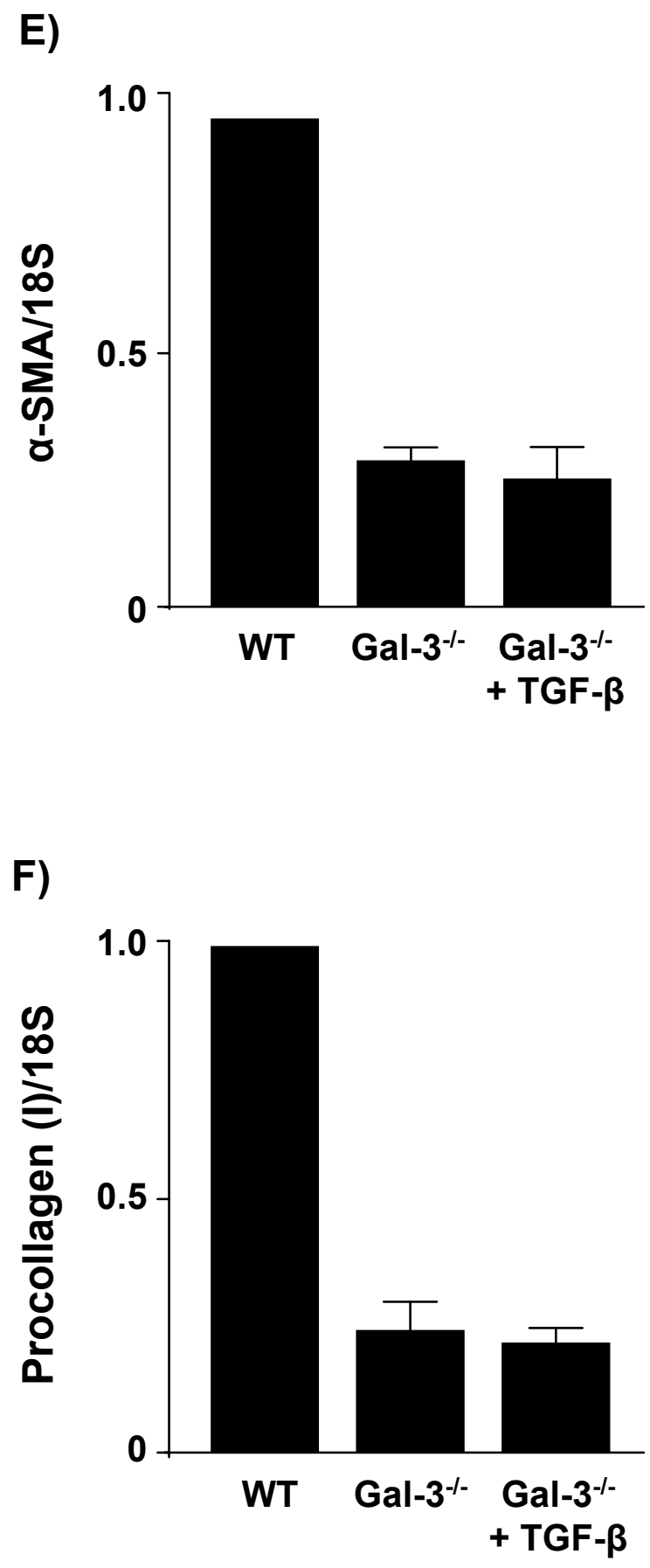

Figure 4.7 (E, F)

(E and F) Real-time RT-PCR quantitation of (E) $\alpha$-SMA expression and (F) procollagen (I) expression (normalised to 18S) in HSCs cultured in the presence of $5 \mathrm{ng} / \mathrm{ml} \mathrm{TGF}-\beta$ for $48 \mathrm{~h}(\mathrm{n}=3)$. Real-time RT-PCR results are expressed as fold change relative to WT. $\mathrm{P}=0.56(\mathrm{~ns})$ and $0.58(\mathrm{~ns})$ for $(\mathrm{E})$ and $(\mathrm{F})$ respectively. 


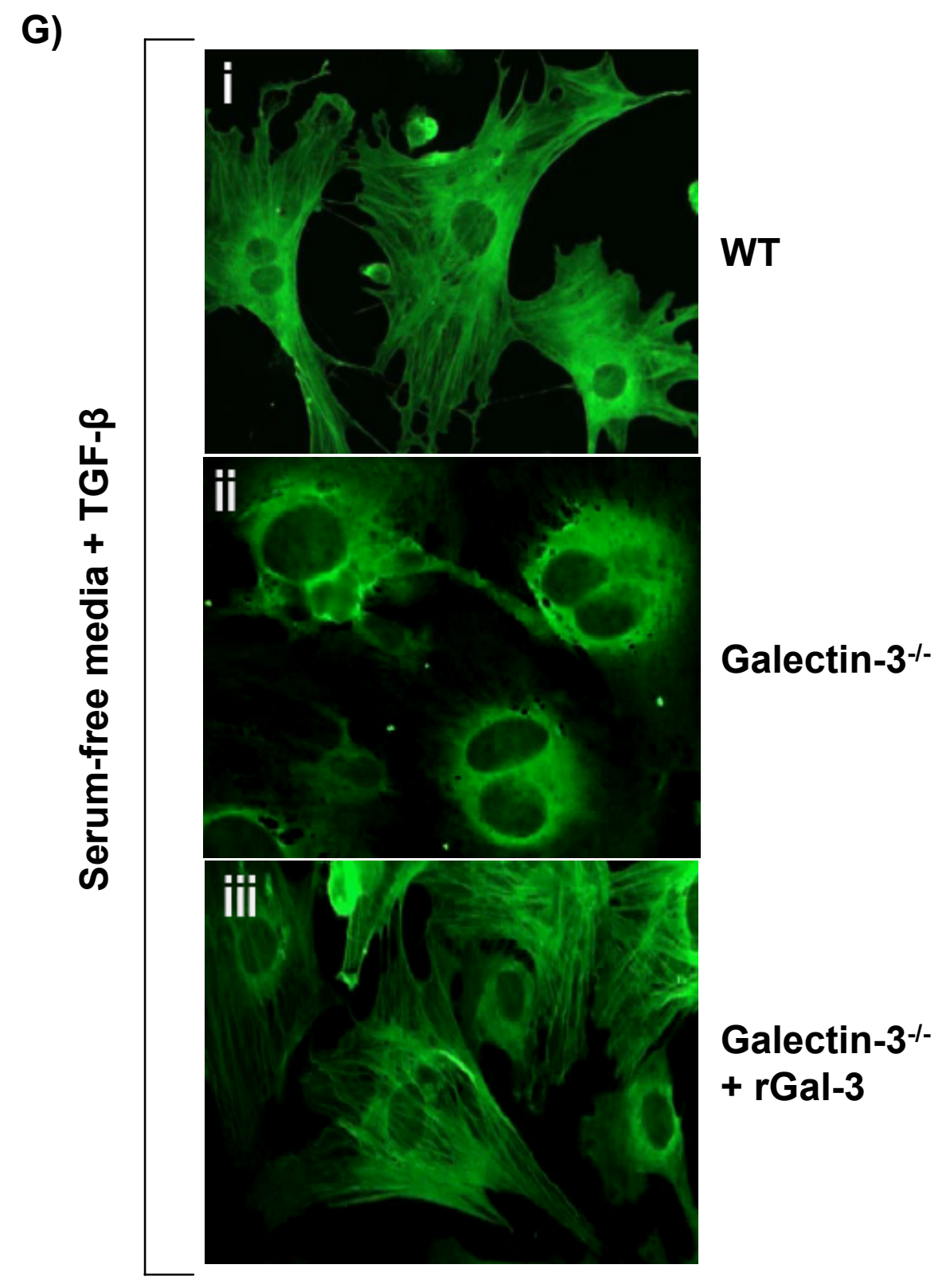

Figure 4.7 (G)

(G) Indirect immunofluoresence for $\alpha$-SMA expression (green) in HSCs (day 4) quiesced in serum-free media for $24 \mathrm{~h}$ and stimulated with $5 \mathrm{ng} / \mathrm{ml}$ TGF- $\beta$ or TGF- $\beta$ plus $30 \mu \mathrm{g} / \mathrm{ml}$ recombinant mouse galectin- 3 for $48 \mathrm{~h}$. 
H)
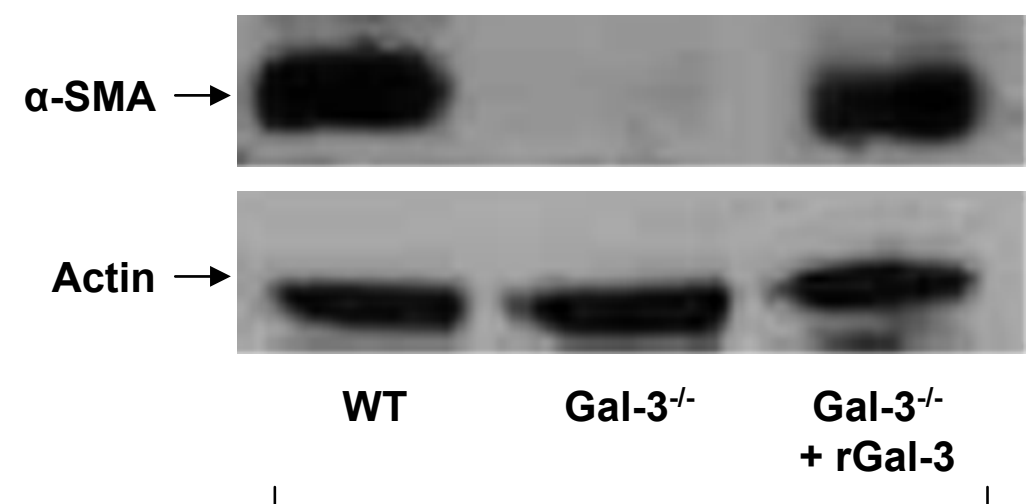

Serum-free media + TGF- $\beta$
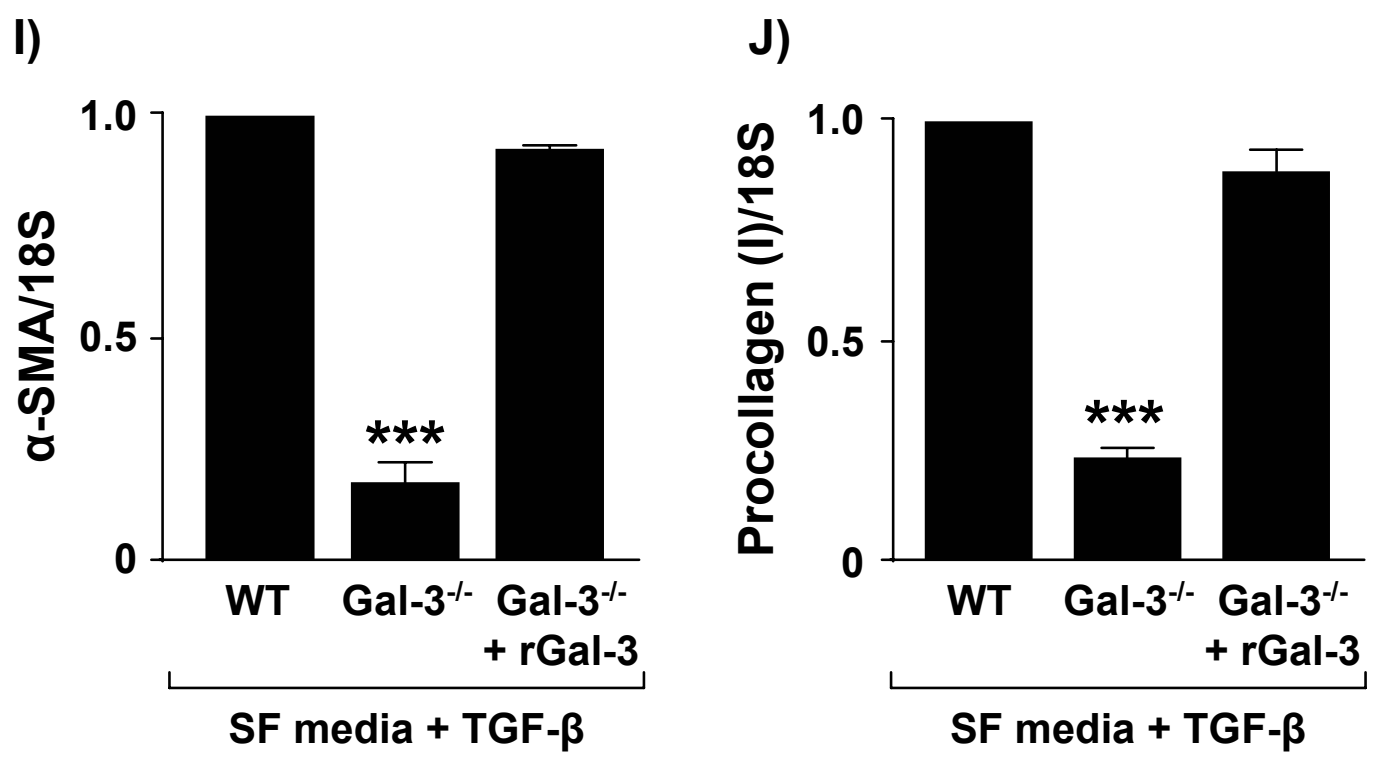

Figure 4.7 (H - J)

(H) Western blot of $\alpha$-SMA expression in lysates from HSCs (day 4) quiesced in serum-free media for $24 \mathrm{~h}$ and stimulated with $5 \mathrm{ng} / \mathrm{ml}$ TGF- $\beta$ or TGF- $\beta$ plus $30 \mu \mathrm{g} / \mathrm{ml}$ recombinant mouse galectin-3 for $48 \mathrm{~h}$. Real-time RT-PCR quantitation of (I) $\alpha$-SMA or (J) procollagen (I) expression (normalised to 18S) in HSCs treated as in $(\mathrm{H}) .{ }^{* * *}, \mathrm{P}<0.0001$ compared to galectin- $3^{-/-}$plus rGal-3 $(\mathrm{n}=3)$. Real-time RT-PCR results are expressed as fold change relative to WT plus TGF$\beta$. 
Previous data indicated that addition of exogenous galectin-3, in the presence of $16 \%$ foetal calf serum (FCS), to galectin- $3^{-/-}$HSCs induced expression of $\alpha$-SMA and procollagen (I) (figure 4.5C and D respectively). To address specifically the contribution of TGF- $\beta$ to this observation the experiments were repeated in serumfree media augmented with $5 \mathrm{ng} / \mathrm{ml}$ recombinant mouse TGF- $\beta$. The galectin- $3^{-/-}$ HSCs still exhibited a reduced activation profile in the presence of TGF- $\beta$ compared with wild type HSCs, as demonstrated by Western blotting for $\alpha$-SMA (figure 4.7D) and real-time RT-PCR quantitation of $\alpha$-SMA (figure 4.7E) and procollagen (I) (figure 4.7F). Incubating with $30 \mu \mathrm{g} / \mathrm{ml}$ exogenous recombinant galectin-3 for 48 hours rescued this defect in galectin- $3^{-/-}$HSCs stimulating an activated wild type morphology and $\alpha$-SMA filament organisation (figure 4.7G). Figure 4.7G (ii) was taken at longer exposure to show the defect in filament organisation of galectin- $3^{-/-}$ HSCs, however, overall $\alpha$-SMA staining was reduced as confirmed by Western blotting for $\alpha$-SMA (figure $4.7 \mathrm{H}$ ) and real-time RT-PCR quantitation of mRNA transcripts for $\alpha$-SMA (figure 4.7I) and procollagen (I) (figure 4.7J). Although TGF$\beta /$ Smad signalling remains unaffected in galectin- $3^{-/-}$HSCs, it isn't enough to cause HSC activation to the myofibroblast phenotype. Although this work demonstrates that galectin-3 is essential for TGF- $\beta$ driven myofibroblast activation, more work needs to be carried out to determine whether galectin-3 signalling and induction of myofibroblast activation is dependent upon TGF- $\beta$. Until then I can hypothesise but not conclude that galectin-3-induced myofibroblast activation occurs through a TGF$\beta$-independent mechanism.

\subsubsection{Galectin-3 does not signal via the MEK/ERK pathway to exert its effects in this model of liver fibrosis}

Platelet-derived growth factor (PDGF) is a key mitogen for fibroblasts, smooth muscle cells and other cells (Maeda et al., 2003; Heldin and Westermark, 1999; Marra et al., 1995). There are various isoforms including PDGF-BB (PDGF with a homodimer of B chains) which has been shown to induce HSC activation and migration in vitro (Ikeda et al., 1999). One signalling pathway of PDGF via its receptor results in a kinase cascade triggering the activation of Raf-1, MEK and 
ERK. ERK has been shown to be important in the activation and proliferation of HSCs and is activated as a result of liver damage in vivo (Marra et al., 1999). It was therefore important to investigate whether galectin-3 utilised this pathway to exert its effects in this model of liver fibrosis.

Maeda et al. (Maeda et al., 2003) previously suggested that galectin-3 dependent ERK-1/2 activation in HSCs was Protein Kinase C (PKC) dependent using a pharmacological inhibitor of PKC. However, Western blotting using antipPKC and anti-pERK1/2 (clone MAPK-YT) antibodies demonstrated no difference in the activation of PKC and ERK1/2 in response to a 10 minute incubation with 50ng/ml PDGF-BB in wild type, galectin- $3^{-/-}$(and knock down with siRNA) HSCs (figure 4.8). 
Figure 4.8 - Galectin-3 does not signal via the MEK/ERK pathway to exert its effects in this model of liver fibrosis.

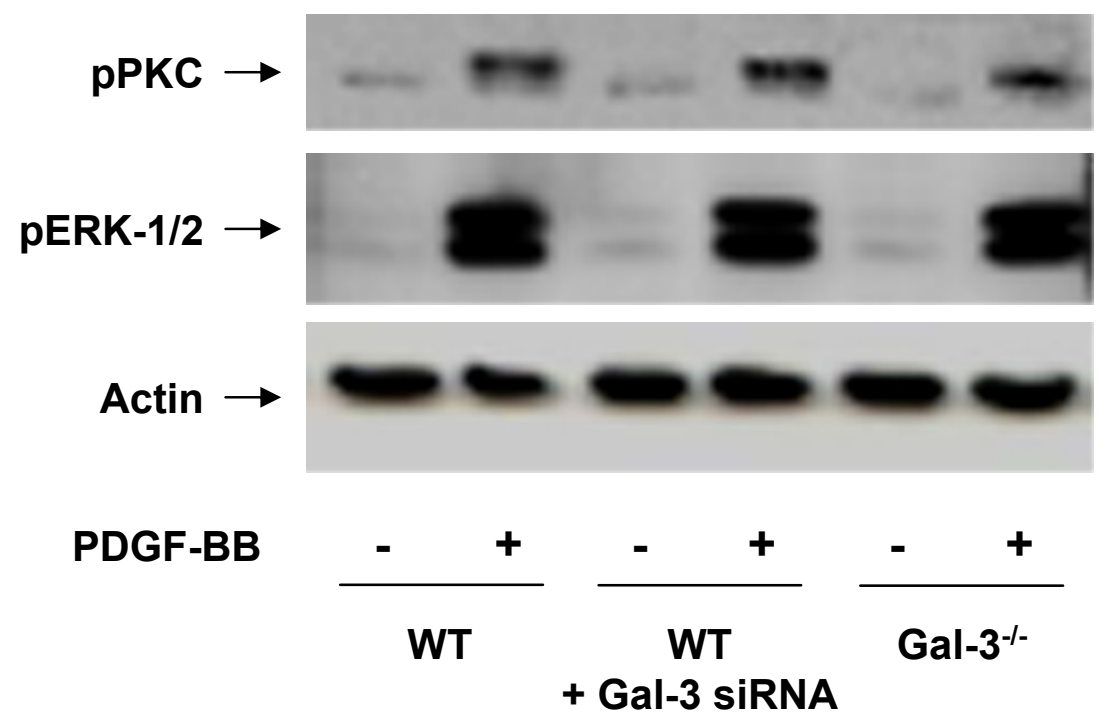

Figure 4.8

Western blot of ERK-1/2 and PKC activation in response to 10 min incubation with 50ng/ml PDGF-BB in WT, 250nM mouse galectin-3 siRNA duplex 1 (96 h), and galectin- $3^{-/}$HSCs. 


\subsubsection{Galectin-3 plays a critical role in kidney fibrosis}

The development of renal fibrosis following UUO was then characterised as an extension of the above work. The role of galectin-3 during renal fibrosis gave similar results as described below.

Following obstruction, kidneys were removed, fixed in methyl carnoys reagent (60\% methanol, 30\% chloroform, 10\% glacial acetic acid) for assessment of macrophage infiltration or formalin for immunohistochemistry. Samples were also snap frozen in liquid nitrogen for real-time RT-PCR analysis.

Hematoxylin and eosin stain $(\mathrm{H} \& \mathrm{E})$ is a general purpose stain particularly used to detect tissue damage and inflammation. Hematoxylin is a basic dye and binds the acidic parts of the cell, i.e. the nucleus, blue. Eosin is an acidic dye and stains the basic parts of the cell, i.e. the cytoplasm, pink-orange. Kidney macrophages were stained with F4/80 (clone CI: A3-1). Obstructed kidneys from wild type and galectin- $3^{-/-}$mice demonstrated equivalent tissue injury (figure 4.9A) and macrophage recruitment (figure $4.9 \mathrm{~B}$ and $\mathrm{C}$ ). Despite this, galectin- $3^{-/-}$mice demonstrated decreased fibrosis, collagen deposition and myofibroblast activation following UUO (figure 4.10). Figure 4.9B inset images represent secondary only control staining (biotinylated anti-rat from the Vector quick kit) of these tissue sections.

Collagen fibres on obstructed kidney sections from wild type and galectin- $3^{-/-}$ mice were stained red with PSR and $\alpha$-SMA was stained using mouse monoclonal anti- $\alpha$-SMA-FITC antibody (clone 1A4). Denser collagen and $\alpha$-SMA staining was observed in kidney sections from wild type compared to galectin- $3^{-/-}$mice (figure 4.10A and B). Figure 4.10B inset image represents secondary only control staining (rabbit anti-FITC) of this tissue section. Staining was quantified using Openlab software for digital image analysis (figure 4.10C and D). Procollagen (I) and $\alpha-S M A$ mRNA expression was analysed by real-time RT-PCR and both were significantly decreased in kidneys from galectin- $3^{-/-}$compared to wild type mice after UUO (figure 4.10E and F). This result suggested that galectin-3 also regulates collagen deposition during renal injury. 
Figure 4.9 - Disruption of the galectin-3 gene does not affect macrophage recruitment following UUO.

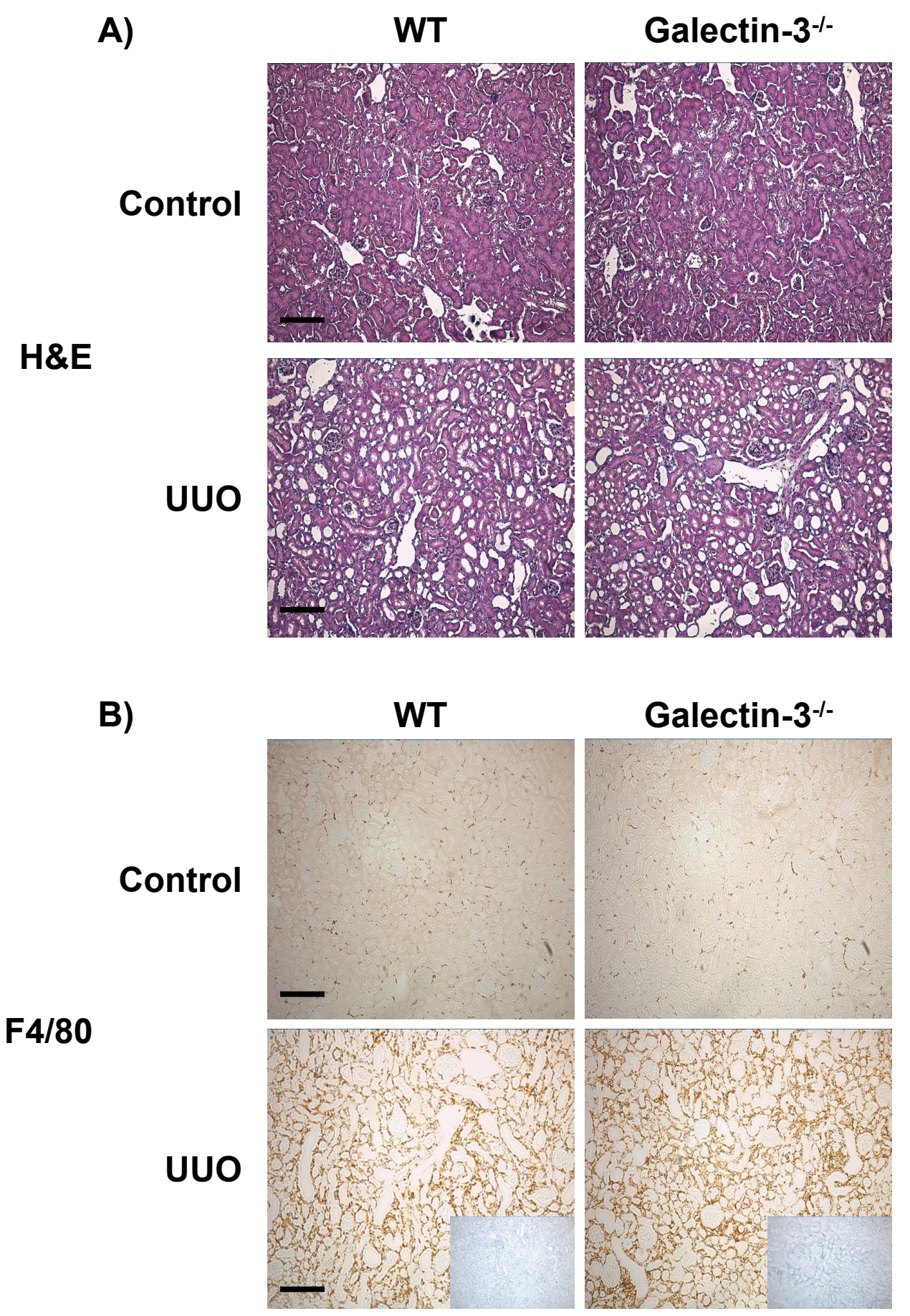

Figure 4.9 (A, B)

(A) Haematoxylin and Eosin (H\&E) staining of kidneys from WT and galectin-3- mice 3 days following sham operation or UUO. (B) Renal macrophages were stained with F4/80. (Scale bar: $100 \mu \mathrm{m}$ ). Inset images demonstrate secondary antibody only control staining. 


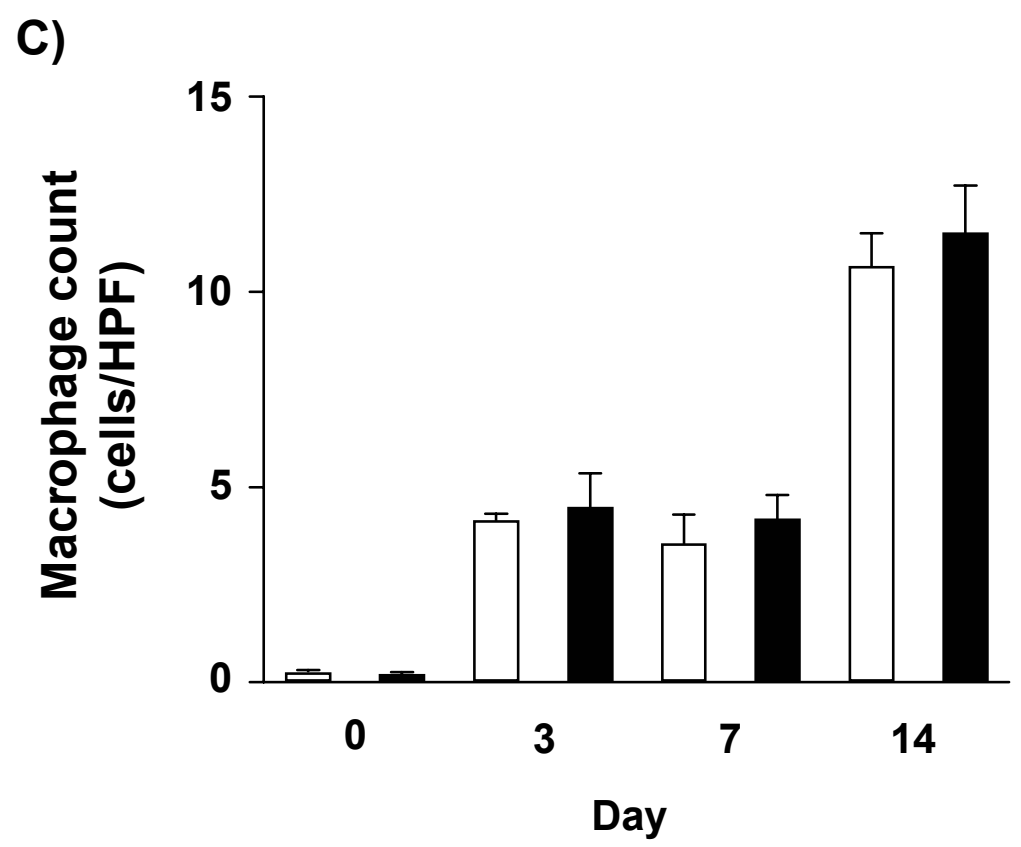

Figure 4.9(C)

(C) WT (white bars) and galectin- $3^{-/}$(black bars) kidneys from day 3, 7 and 14 days post-UUO were fixed and stained for F4/80. Numbers of F4/80 positive macrophages per high power field ( $\mathrm{x} 5$ from each section) were counted. $(\mathrm{n}=3$ mice in each group). $\mathrm{P}=0.49$ (ns), 0.33 (ns) and 0.37 (ns) for day 3, 7 and 14 respectively. 
Figure 4.10 - Absence of galectin-3 protects against renal fibrosis.
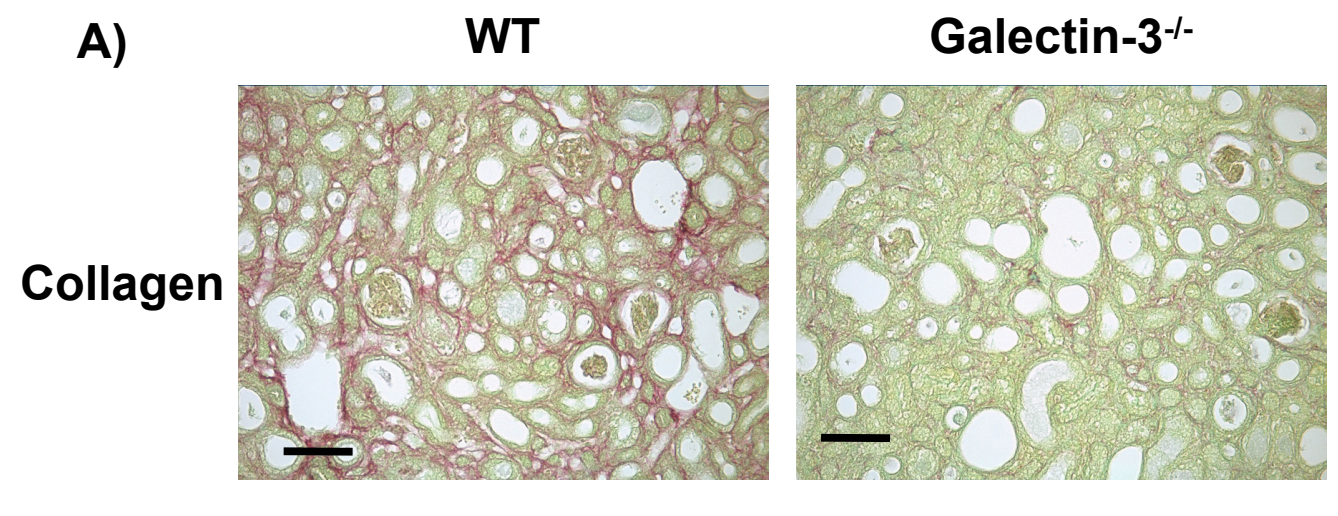

B)
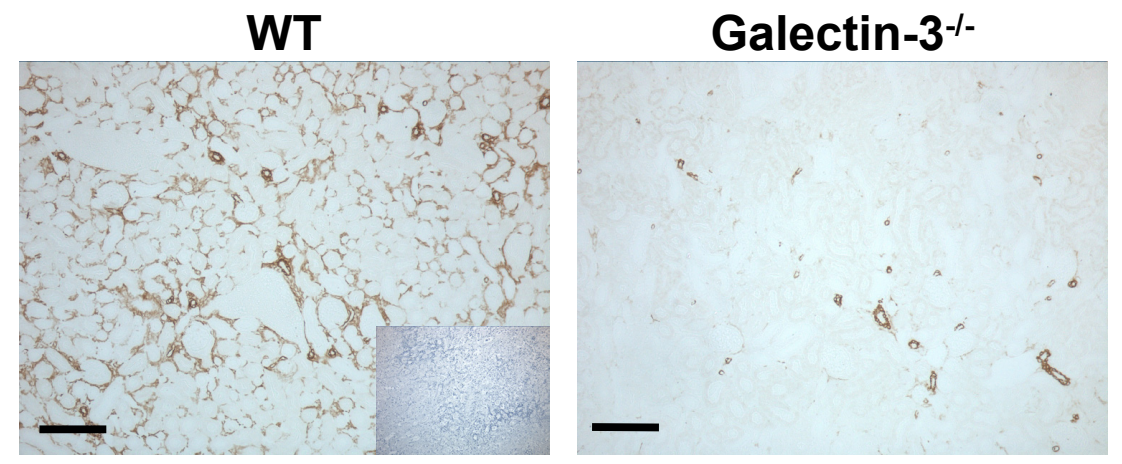

Figure 4.10 (A, B)

Mice underwent control (sham operation) or UUO and were sacrificed at 7 days ( $\mathrm{n}=6$ mice in each group) (A) Renal collagen deposition in WT and galectin- $3^{-/-}$ kidneys after UUO was examined by PSR staining. (B) $\alpha$-SMA staining in WT and galectin- $3^{-/-}$kidneys after UUO. (Scale bar: $100 \mu \mathrm{m}$ ). Inset image demonstrates secondary antibody only control staining. 

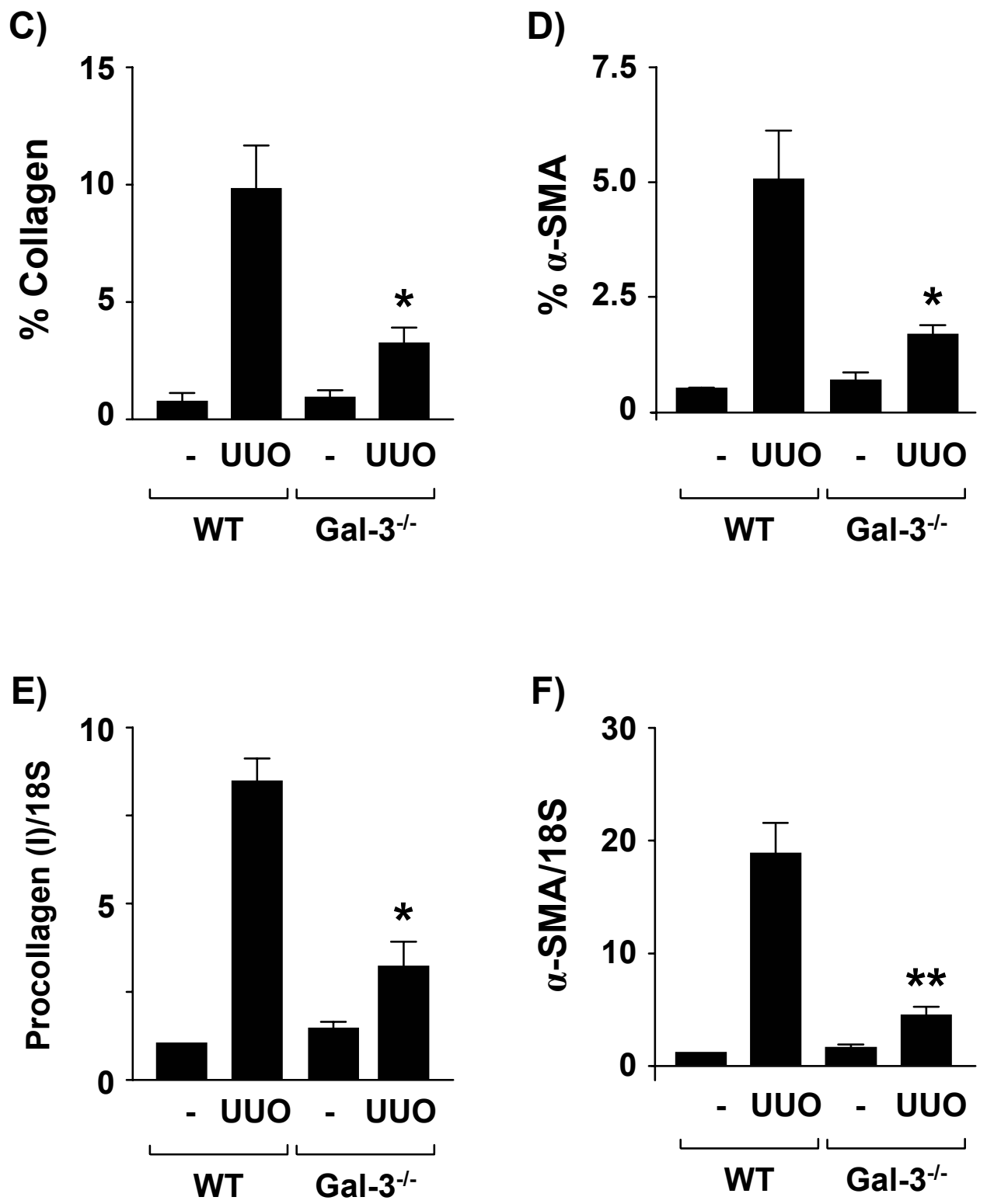

\section{Figure 4.10 (C-F)}

Digital image analysis quantitation of collagen (C) and $\alpha$-SMA (D) staining. *, P $<0.05$ compared to WT UUO. (E) Real-time RT-PCR quantitation of procollagen (I) expresion (normalised to 18S) in total RNA extracts from control kidneys and obstructed kidneys from WT and galectin $-3^{-/ 2}$ mice. ${ }^{*}, \mathrm{P}<0.05$ compared to WT UUO. (F) Real-time RT-PCR quantitation of $\alpha$-SMA expression (normalised to 18S) in total RNA extracts from control kidneys and obstructed kidneys from WT and galectin- $3^{-/-}$mice. ${ }^{* *}, \mathrm{P}<0.01$ compared to WT UUO. Real-time RT-PCR results are expressed as fold change relative to WT control. 


\subsection{Discussion}

This study has shown a fundamental role for galectin-3 in the regulation of HSC activation in vitro and in vivo, thereby identifying galectin-3 as a potential therapeutic target in the treatment of liver fibrosis. This work has also shown that this may be a pan-organ effect as shown by a reduction of renal fibrosis following UUO in galectin-3 deficient mice.

Human biopsy data demonstrated that galectin-3 expression is increased in human liver fibrosis secondary to diverse types of injury including viral-mediated (Hepatitis B and C) and alcohol-induced. This suggested that galectin-3 upregulation is a basic response within the liver regardless of the initiating agent or disease process. The pattern of galectin-3 staining observed in the human tissue was different from the distribution observed in the animal models. This difference related to the chronicity and intensity of the disease processes. Human tissue was taken from patients with advanced cirrhosis following years (in many cases decades) of chronic injury and fibrosis. In the mouse and rat models a much shorter time-course of injury ( 8 and 12 weeks respectively) leading to liver fibrosis was examined.

The experimental model of liver fibrosis showed a very close spatial and temporal relationship between galectin-3 expression, myofibroblast activation and collagen deposition. Galectin-3 can be considered an immediate early gene and is upregulated rapidly in response to tissue injury (Kadrofske et al., 1998; Chiariotti et al., 2004). These results demonstrated that spontaneous HSC activation occured in wild type but not galectin- $3^{-/-}$HSCs and that this defect could be overcome by exogenous addition of galectin-3 (which was rapidly internalised by HSCs). Spontaneous activation of wild type HSCs was blocked by siRNA knockdown of galectin-3 expression. There is perhaps a galectin-3 autocrine stimulation of HSCs causing HSC activation in vitro. However, the peri-sinusoidal orientation and long cytoplasmic processes of HSCs facilitate their interactions with neighboring cell types including other non-parenchymal cells such as kupffer cells and sinusoidal endothelial cells and liver parenchymal cells (hepatocytes). This may regulate HSC phenotype and function by facilitating both autocrine and paracrine activation of myofibroblasts by galectin-3 via cell-cell contacts, cell-matrix contacts and via soluble factors. Furthermore, within the injured liver, injured epithelium 
(hepatocytes) upregulate galectin-3 expression following injury and both recruited and resident tissue macrophages are abundant sources of galectin-3 (Sato and Hughes, 1994). Thus both autocrine and paracrine galectin-3 stimulated HSC activation may exist during liver inflammation and fibrosis in vivo.

TGF- $\beta$ is a major pro-fibrogenic cytokine and is a key mediator of fibrosis in many different organs (Leask and Abraham, 2004). TGF- $\beta$ mRNA expression was markedly elevated following hepatic injury, however expression of TGF- $\beta$ was similar in whole liver homogenates from fibrotic liver in wild type and galectin- $3^{-/-}$ mice. Secretion of TGF- $\beta$ was the same in wild type and galectin- $3^{-/-}$HSCs, and Smad-2 and Smad-3 signalling in HSCs was similar between the two genotypes when stimulated with TGF- $\beta$. However, despite similar levels of TGF- $\beta$ and intact TGF- $\beta$ signalling pathways, the absence of galectin-3 markedly inhibited the fibrotic phenotype in vitro and in vivo in the animal model. This would suggest that TGF- $\beta$ stimulated HSC activation and procollagen production requires galectin-3.

Galectin-3 can form pentamers in the presence of multivalent ligands, crosslinking glycoproteins at the cell membrane (Ahmad et al., 2004). Mgat5-modified Nglycans on cell-surface receptors such as the epidermal growth factor receptor can be crosslinked by galectin-3 (Partridge et al., 2004) thus regulating receptor cell surface expression and activity leading to effects on intracellular signalling. The time-course experiments examining trafficking of exogenous recombinant galectin-3 added to primary HSCs suggest that galectin-3 is rapidly internalised. Furthermore, siRNA mediated knockdown of galectin-3 inhibited myofibroblast activation and procollagen expression. Intracellularly, galectin-3 can shuttle between the nucleus and the cytoplasm (Davidson et al., 2002) and is involved in fundamental processes such as pre-mRNA splicing (Dagher et al., 1995; Wang et al., 2004), cell-cycle progression (Kim et al., 1999; Lin et al., 2002), proliferation (Moutsatsos et al., 1987; Inohara et al., 1998; Shimura et al., 2004), and apoptosis (Yang et al., 1996; Akahani et al., 1997; Honjo et al., 2001; Yu et al., 2002) mainly through intracellular proteinprotein interactions rather than lectin-carbohydrate interactions. However the precise mechanisms by which galectin- 3 regulates these intracellular processes have still to be defined. Recently it has been shown that TGF- $\beta$ can induce renal fibrosis in a Smad-2/3-independent fashion (Moustakas and Heldin, 2005; Wang et al., 2005) and 
activates additional signalling molecules such as p38 (Tsukada et al., 2005), bcr-abl (Wang et al., 2005) and PAK2 (Wilkes et al., 2003). The present work is consistent with the hypothesis that TGF- $\beta$ induced fibrosis requires galectin-3 and includes a mechanism that is independent of Smad-2 and Smad-3. However, to fully test this hypothesis, more work needs to be done to ascertain that galectin-3-induced myofibroblast activation occurs through a TGF- $\beta$-independent mechanism.

In addition to this work carried out using the model of liver fibrosis, the UUO model of renal fibrosis demonstrated similar in vivo results. As with the liver, galectin- $3^{-/-}$mice demonstrated reduced renal fibrosis following UUO with reduced fibroblast activation as demonstrated by diminished $\alpha$-SMA and procollagen (I) staining and mRNA levels despite comparable recruitment of macrophages. This suggests that galectin-3 plays an important role in the progression of fibrosis in multiple organ systems.

RNA interference allows in-depth study of the molecular mechanisms of disease through specific gene target inhibition. Furthermore, siRNAs hold direct therapeutic promise, as agents capable of attenuating the expression of diseasecausing genes (Soutschek et al., 2004). siRNA duplexes were used to examine the role of galectin-3 in myofibroblast activation in vitro. siRNA silencing of galectin-3 expression in mouse HSCs resulted in inhibition of myofibroblast activation and procollagen (I) expression. Strategies to knockdown expression of galectin-3 in the liver may lead to the development of novel anti-fibrotic therapies.

Additional work from this laboratory has shown that administration of galectin-3 siRNA via the portal vein in vivo reduced HSC activation in response to $\mathrm{CCL}_{4}$ (Henderson et al., 2006). In addition, preliminary work by N. Henderson has demonstrated a decrease in in vivo fibrosis following UUO by administration of galectin-3 siRNA via the renal artery (unpublished). 


\section{Chapter 5}

\section{Regulation of alternative macrophage activation by galectin-3}

\subsection{Introduction}

Macrophages display broad phenotypic heterogeneity depending on their microenvironment (Gordon, 2003; Stout et al., 2005; Gordon et al., 1992). During an inflammatory response, the microenvironment changes from an initial proinflammatory/cytotoxic environment to an anti-inflammatory/tissue regenerative environment and the macrophage adapts to its surroundings by changing its functional phenotype. The plasticity of macrophages enables the activation status to be skewed to an M1 or an M2 phenotype with the addition of Th1 or Th2 cytokines respectively.

The initial inflammatory response is predominantly mediated by classically activated (M1 polarised) macrophages, which eradicate invading organisms and tumour cells (Mantovani et al., 2002; Munder et al., 1998). The pro-inflammatory and cytotoxic activities of classically activated M1 macrophages are enhanced in the presence of microbial agents endotoxin (Lipopolysaccharide - LPS) and/or Th1 cytokines such as Interferon- $\gamma$ (IFN $\gamma$ ) or Interleukin-12 (IL-12) (Holscher et al., 2001; Louis et al., 1998). Classical activation by IFN $\gamma$ in particular is associated with nitric oxide synthetase 2 (NOS2) expression and the production of large amounts of nitric oxide and pro-inflammatory cytokines including Tumour necrosis factor- $\alpha$ (TNF- $\alpha$ ) and IL-6 (Munder et al., 1998; Munder et al., 1999; Welch et al., 2002). Upon classical activation, macrophages release proinflammatory cytokines, and the metabolism of L-arginine is driven towards the production of nitrite via the enzyme NOS2 (Hesse et al., 2001).

In contrast, the resolution phase of inflammation is driven by alternatively activated (M2 polarised) macrophages, which are hypo-responsive to proinflammatory stimuli. Macrophages undergo alternative activation when stimulated with the Th2 cytokines, IL-4 or IL-13 (Gordon, 2003; Munder et al., 1999; Hesse et al., 2001). Treatment of murine macrophages with IL-4 or IL-13 causes upregulation 
of mannose receptor (Stein et al., 1992), arginase-1 (Hesse et al., 2001), YM-1 (chitinase-like lectin) (Nair et al., 2005; Raes et al., 2002) and FIZZ-1 (resistin-like secreted protein) expression (Herbert et al., 2004; Nair et al., 2003). Upon alternative activation, the metabolism of L-arginine is driven towards the production of L-ornithine and pro-fibrotic products via the enzyme arginase-1 (Hesse et al., 2001) (Figure 1.4).

Initially it was believed that IL-4 and IL-13 resulted in a deactivated phenotype similar to that seen with IL-10, causing deactivation of the respiratory burst and of inflammatory cytokine production, particularly TNF- $\alpha$, facilitating the elimination of infectious organisms with minimal damage to host tissues (Moore et al., 2001). However, IL-10 acts on a distinct plasma membrane receptor to IL-4 and IL-13 and the regulatory role of IL-4 and IL-13 on immune responses appear to be more complex than first thought (D'Andrea et al., 1995; Gordon, 2003).

Alternative (M2) macrophage activation has been implicated in diverse disease pathologies in the host response to parasitic infection, asthma, wound repair, atheromatous plaques and tumour-associated macrophages (Herbert et al., 2004; Nair et al., 2003; Raes et al., 2005; Wynn, 2004; Sica et al., 2006). Furthermore, the alternatively activated (M2) macrophage plays an important role in certain fibrotic diseases (Wynn, 2004) such as experimental silicosis (Misson et al., 2004), herpesvirus-induced lung fibrosis (Mora et al., 2006) and hepatic fibrosis (Hesse et al., 2001; Chiaramonte et al., 2001; Reiman et al., 2006). Inhibitors of alternative macrophage activation may restrict fibrosis in granuloma formation (Hesse et al., 2001) and enhance host immunity against cancers (Sica et al., 2006). In disease states where alternatively activated macrophages limit tissue injury or promote repair, it might be helpful to augment their activity, for example in stabilising atherosclerotic plaques. In order to exploit M1 and M2 macrophages for future antiinflammatory and anti-cancer therapies it is important to understand the mechanism and extracellular ligands that determine M1 and M2 macrophage programming.

Galectin-3 is highly expressed and secreted by macrophages and recent data suggests that galectin-3 plays a significant role in many facets of macrophage biology. Galectin-3 is up-regulated when monocytes differentiate into macrophages (Liu et al., 1995) and down-regulated when macrophages differentiate into 
dendriticcells (Dietz et al., 2000). Galectin-3 also promotes monocyte-monocyte interactions that ultimately lead to polykaryon (multinucleated giant cell) formation, a phenotype associated with chronic inflammatory and fibrotic diseases (Okamoto et al., 2003). Previous work detailed in chapter 4 of this thesis has demonstrated that mice deficient in galectin-3 exhibit reduced hepatic fibrosis following chronic administration of carbon tetrachloride $\left(\mathrm{CCl}_{4}\right)$ (Henderson et al., 2006). CD98 is a disulfide-linked $125 \mathrm{kDa}$ heterodimeric type II transmembrane glycoprotein composed of a glycosylated $85 \mathrm{kDa}$ heavy chain (designated CD98) and a nonglycosylated 40-kDa light chain (Verrey et al., 2004). CD98 is highly expressed on macrophages and has been shown to be a receptor for galectin-3 (Dong and Hughes, 1997; Hughes, 2001; Dong and Hughes, 1996). Furthermore crosslinking CD98 with anti-CD98 monoclonal antibodies promotes polykaryon formation (Tajima et al., 1999). Therefore modulation of galectin-3 expression and interaction with CD98 during macrophage differentiation may be important in the regulation of macrophage plasticity and control of macrophage fate.

Chapter 4 of this thesis described galectin-3 playing a critical role during fibrosis, stimulating myofibroblast activation, resulting in collagen production and the enhancement of fibrosis (Henderson et al., 2006). As highlighted above, alternatively activated macrophages have been implicated as important augmenters of fibrosis. It was therefore hypothesised that galectin-3 is involved in alternative macrophage activation thus contributing to the progression of fibrosis. This chapter aims to investigate the importance of galectin-3 in alternative macrophage activation and presents preliminary work investigating the role of the alternatively activated macrophage during the unilateral ureteric obstruction (UUO) model of renal fibrosis. 


\subsection{Results.}

\subsubsection{Disruption of the galectin-3 gene in macrophages causes a specific defect in alternative activation of in vitro and in vivo differentiated macrophages}

To investigate the role of galectin-3 in macrophage activation, in vitro derived bone marrow derived macrophages (BMDMs) from wild type and galectin- $3^{-}$ ${ }^{1-}$ mice were used. BMDMs were flushed from the femurs and tibias of wild type and galectin- $3^{-/-}$mice and matured for 7 days in media containing 10\% L929 conditioned media as a source of granulocyte-macrophage colony-stimulating factor (GMCSF). Maturity was examined by dual immunofluorescence staining for CD11b and F4/80 and analysed by flow cytometry. Macrophages were incubated with a 1:150 dilution of CD11b-PE antibody (clone M1/70) and a 1:25 dilution of F4/80-FITC antibody (clone CI:A3-1) for 30 minutes at room temperature in the dark. Samples were washed and a 1:10,000 dilution of the vital dye ToPro-3 was added to each tube just prior to flow cytometry analysis. Wild type and galectin- $3^{-/}$BMDM matured to equal extents as measured by F4/80 and CD11b expression (figure 5.1A) and were over $95 \%$ viable (figure $5.1 \mathrm{~B}$ ).

Classical macrophage (M1) activation was assessed through the addition of IFN $\gamma /$ LPS and alternative (M2) activation measured via the addition of IL-4 or IL-13. The following methods were performed to determine the macrophage activation status. Complete methods are given in the Materials and Methods chapter. The proinflammatory cytokines TNF- $\alpha$ and IL-6 were measured using the cytometric bead array (CBA) mouse inflammation kit from BD Biosciences. Nitric oxide (NO) production was measured using the Griess reaction first described in 1879 (Griess P, 1879). Nitric oxide is very unstable and rapidly oxidises to nitrite $\left(\mathrm{NO}_{2}\right)$ and nitrate $\left(\mathrm{NO}_{3}\right)$ by oxygen. The Griess reagent reacts with the stable oxidation product nitrite to produce an intense purple colour which can be measured at 540nm. Arginase-1 activity was measured by activating the arginase- 1 in the cell lysates (prepared using lysis buffer containing EDTA-free protease inhibitors) with manganese chloride and incubating the samples with the substrate L-arginine. 
Figure 5.1 - Disruption of the galectin-3 gene does not affect BMDM maturation.
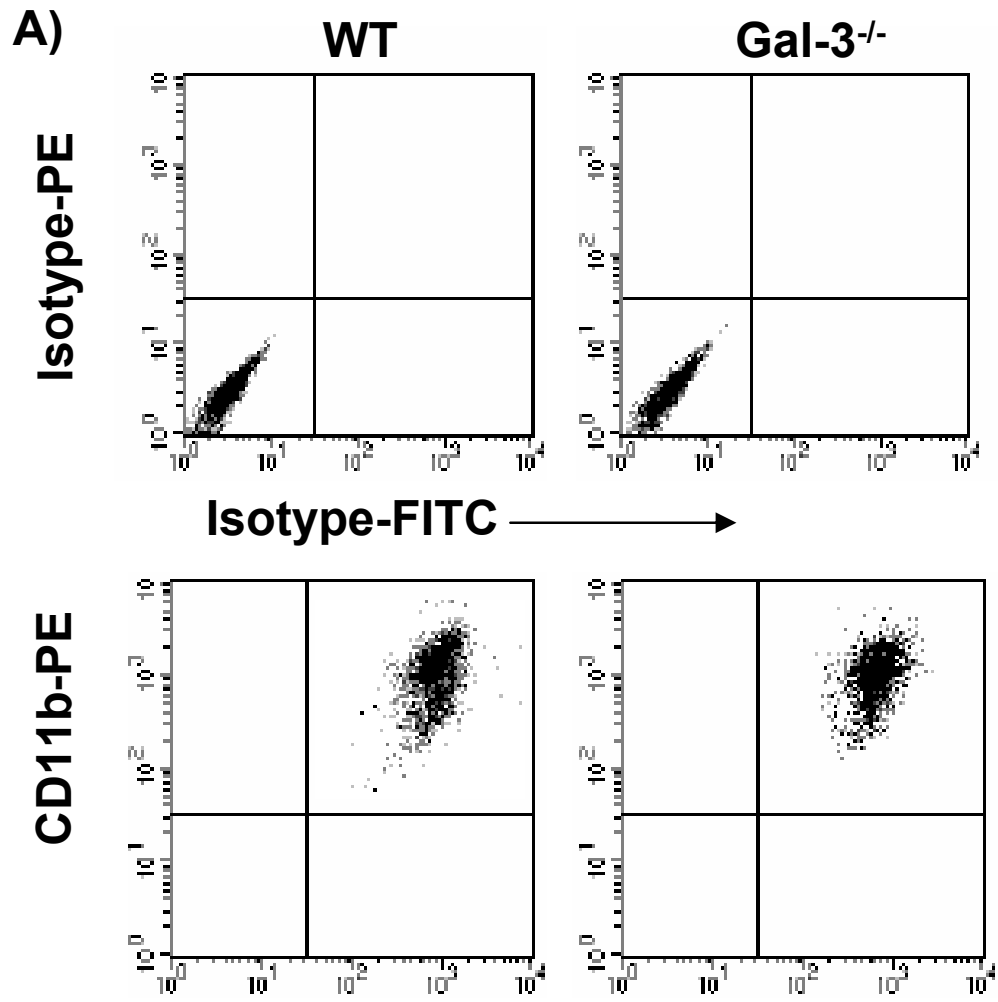

F4/80-FITC

B)

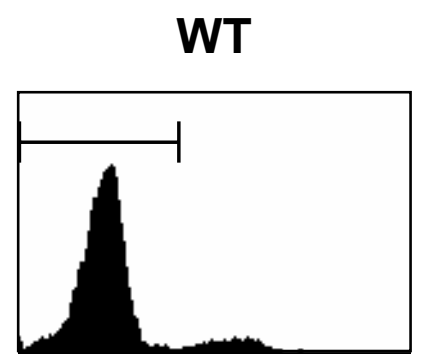

Gal-3-1-

FL-4 (ToPro-3)

Figure 5.1 (A, B)

(A) FACS characterisation of $\mathrm{WT}$ and galectin- $3^{-/-}$bone marrow-derived macrophages (BMDMs) matured for 7 days using 1:25 dilution of F4/80-FITC and 1:150 dilution of CD11b-PE antibodies added for $30 \mathrm{~min}$ at room temperature in the dark. (B) BMDM viability was measured by topro-3 exclusion. 1:10,000 dilution of the vital dye ToPro-3 was added to each tube just prior to FACS analysis. 
An acid mixture was added to stop the reaction and the urea formed was quantified after the addition of $\alpha$-isonitrosopropiophenone (ISPF).

Following treatment with $100 \mathrm{U} / \mathrm{ml} \mathrm{IFN \gamma}$ and $100 \mathrm{ng} / \mathrm{ml}$ LPS, wild type and galectin- $3^{-/-}$macrophages produced similar levels of the proinflammatory cytokines TNF- $\alpha$ and IL-6 (figure 5.2A and B) indicating equal ability to classically activate. Incubation with $10 \mathrm{ng} / \mathrm{ml}$ IL-4 or IL-13 alone had no effect on cytokine release (figure 5.2A and B) but significantly reduced LPS-stimulated TNF- $\alpha$ and IL-6 release in wild type but not galectin- $3^{-/-}$macrophages (figure $5.2 \mathrm{C}$ and $\mathrm{D}$ ).

Classical activation with IFN $\gamma$ and LPS produced a similar increase in nitrite production from both wild type and galectin- $3^{-/-} \mathrm{BMDM}$ (figure 5.2E). Alternative activation of wild type BMDMs with IL-4 and IL-13 induced a $>5$ fold stimulation in arginase-1 activity over untreated macrophages. However IL-4 and IL-13 treated galectin- $3^{-/-}$BMDMs induced significantly less arginase-1 activity when compared to wild type BMDMs (figure 5.2F and figure 5.3A). True deactivation, induced by IL10 , inhibited TNF- $\alpha$, IL-6 and nitrite release by LPS in both wild type and galectin-3 /- BMDMs (figure 5.2C, D and E) suggesting that galectin-3 is not essential for IL10-induced macrophage deactivation.

SYBR Green real-time RT-PCR analysis of untreated, IL-4- or LPSstimulated BMDMs showed equal expression of the IL-4/IL-13 common receptor, IL-4R $\alpha$, in wild type and galectin- $3^{-/-}$macrophages (figure 5.3B). To further characterise alternative activation in response to IL-4, BMDMs were treated with IL4 in the presence or absence of LPS and markers of alternative activation were analysed by SYBR Green real-time RT-PCR. IL-4 produced a significant increase in the ratio of arginase/NOS2 expression in wild type BMDMs indicating a skew to the M2 phenotype. However, the increased ratio was significantly less in galectin- $3^{-/-}$ BMDMs (figure 5.3C). LPS alone caused a marked increase in NOS2 expression, decreasing the arginase/NOS2 ratio and this was not significantly different between wild type and galectin- $3^{-/}$BMDMs (figure 5.3C). Expression of mannose receptor (figure 5.3D), YM-1 (figure 5.3E) and FIZZ-1 (figure 5.3F) significantly increased in response to IL-4 in wild type BMDMs. However, galectin- $3^{-/}$macrophages demonstrated significantly less increase in the expression of these alternative activation markers in response to IL-4 (figure 5.3D-F). 
Figure 5.2 - Alternative macrophage activation by IL-4 and IL-13 is abrogated in galectin-3-l- BMDMs.

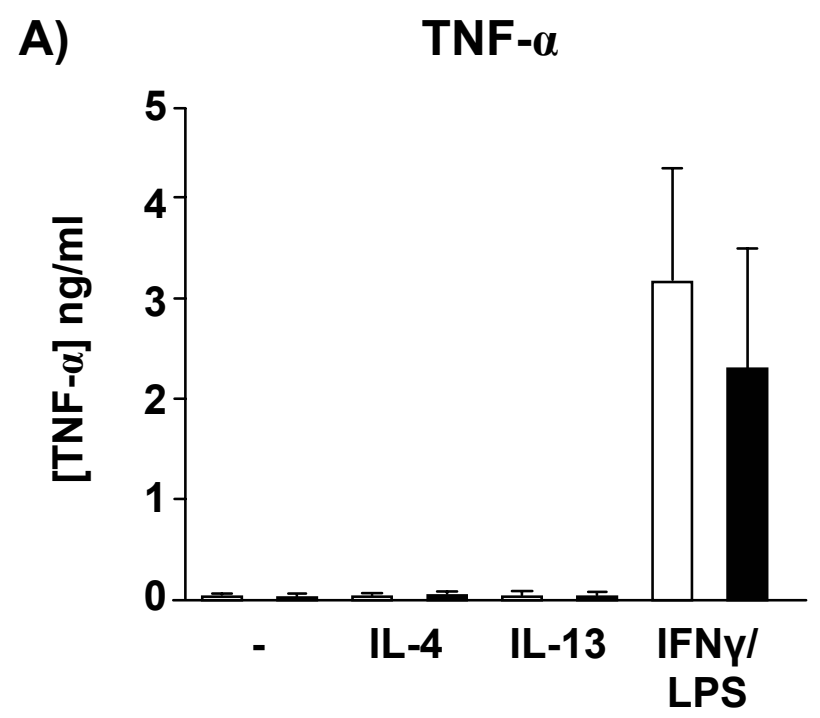

B) IL-6

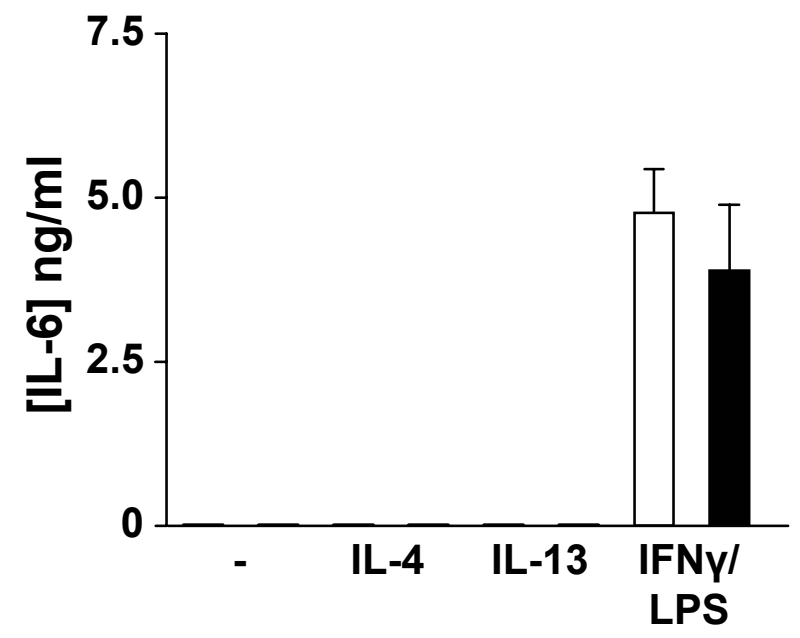

Figure 5.2 (A, B)

WT (white bars) or galectin-3 ${ }^{-/}$BMDMs (black bars) were cultured in the presence of IL-4, IL-13 $(10 \mathrm{ng} / \mathrm{ml})$ or IFN $\gamma(100 \mathrm{U} / \mathrm{ml})$ for $48 \mathrm{~h}$. Where indicated, LPS $(100 \mathrm{ng} / \mathrm{ml})$ was added for the last $24 \mathrm{~h}$. Supernatants were harvested and analysed for TNF- $\alpha$ (A) and IL-6 (B) by cytometric bead array (CBA) $(\mathrm{n}=3)$. For IFN $\gamma /$ LPS treatment $\mathrm{P}=0.66(\mathrm{~ns})$ and $0.06(\mathrm{~ns})$ for $(\mathrm{A})$ and $(\mathrm{B})$ respectively. 


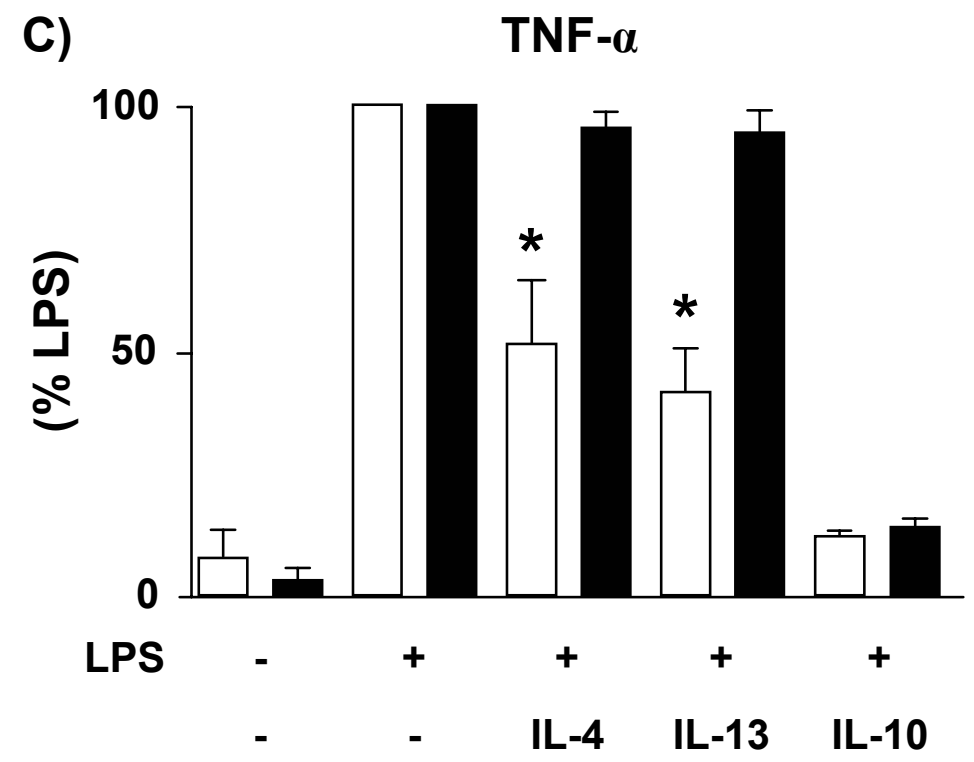

D) IL-6

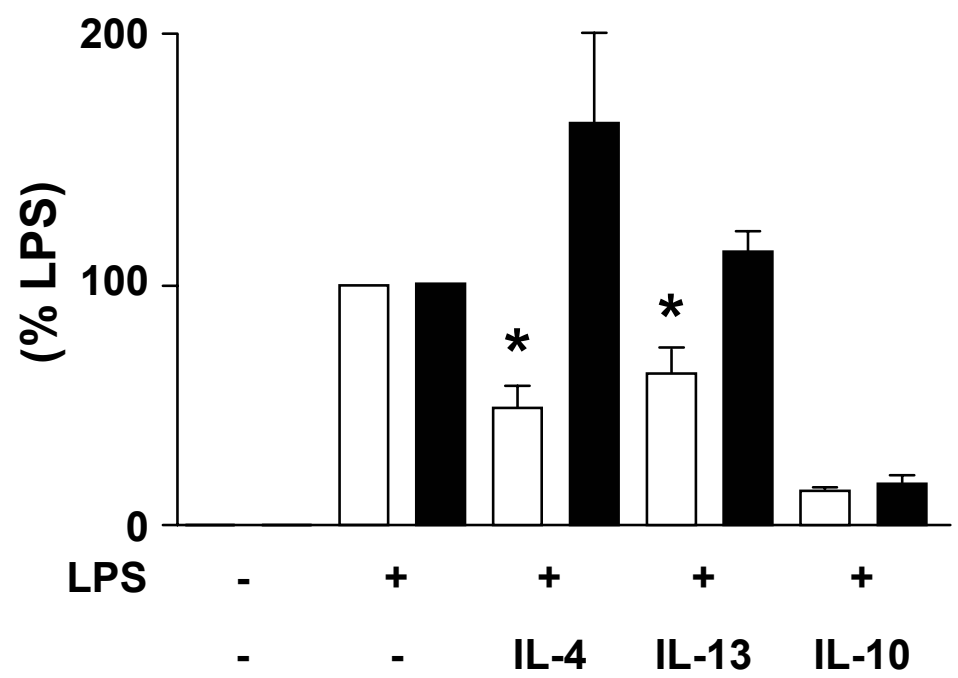

Figure 5.2 (C, D)

WT (white bars) or galectin- $3^{-/}$BMDMs (black bars) were cultured in the presence of IL-4, IL-13 or IL-10 $(10 \mathrm{ng} / \mathrm{ml})$ for $48 \mathrm{~h}$. Where indicated, LPS $(100 \mathrm{ng} / \mathrm{ml})$ was added for the last $24 \mathrm{~h}$. Supernatants were harvested and analysed for TNF- $\alpha$ (C) and IL-6 (D) by cytometric bead array $(\mathrm{n}=3)$. *, P < 0.05 compared to LPS alone. For IL-10 + LPS treatment P $=0.07$ (ns) for both (C) and (D). 


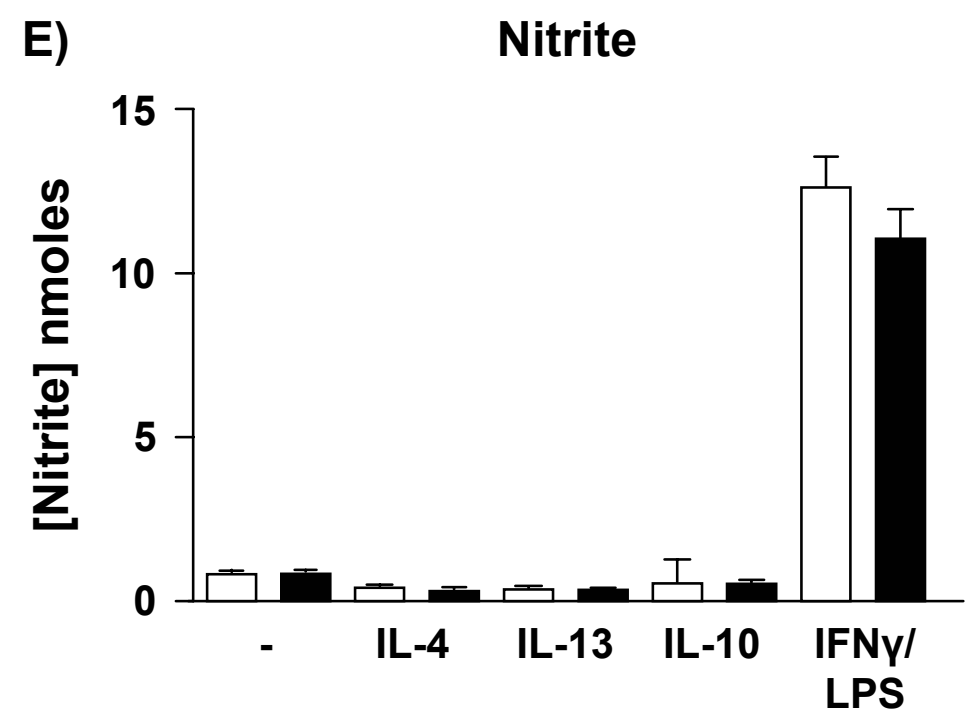

F)

Arginase

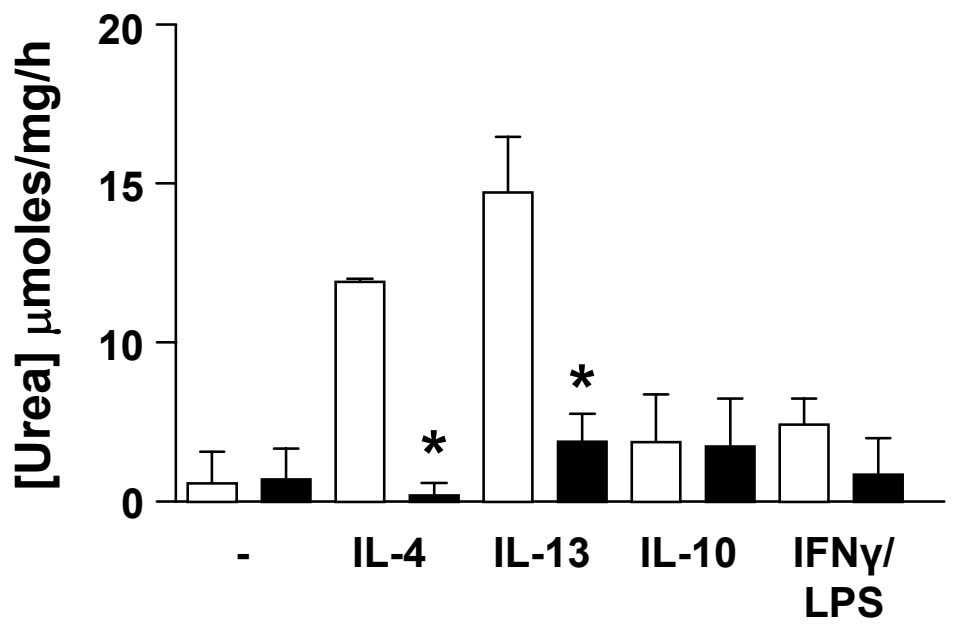

Figure 5.2 (E, F)

WT (white bars) or galectin- $3^{-/}$BMDMs (black bars) were cultured in the presence of IL-4, IL-13, IL-10 $(10 \mathrm{ng} / \mathrm{ml})$ or IFN $\gamma(100 \mathrm{U} / \mathrm{ml})$ for $48 \mathrm{~h}$. Where indicated, LPS $(100 \mathrm{ng} / \mathrm{ml})$ was added for the last $24 \mathrm{~h}$. (E) Nitrite release into supernatants was measured as described in Materials and Methods. For IFN $\gamma / \mathrm{LPS}$ treatment $\mathrm{P}=0.13$ (ns). (F) Arginase activity in cell lysates was measured as described in Materials and Methods $(\mathrm{n}=3)$. ${ }^{*}, \mathrm{P}<0.05$ compared to WT. $\mathrm{P}=$ $0.89(\mathrm{~ns})$ and $0.12(\mathrm{~ns})$ for IL-10 and IFN $\gamma / \mathrm{LPS}$ treatment respectively. 
Figure 5.3 - IL-4 and IL-13 induced alternative activation is reduced in galectin-3-- BMDMs.

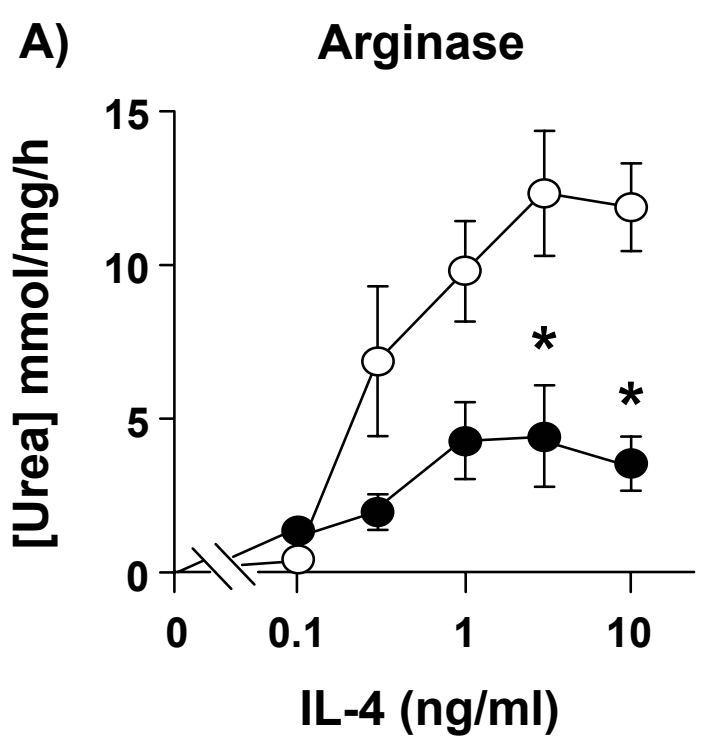

B) IL-4Ra

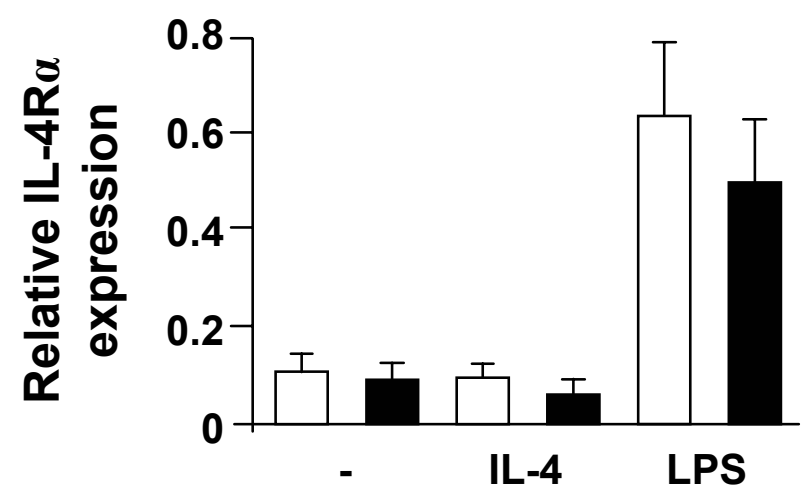

Figure 5.3 (A, B)

(A) WT (white circles) or galectin- $3^{-/}$(black circles) BMDMs were treated with IL-4 $(0-10 \mathrm{ng} / \mathrm{ml})$ for $24 \mathrm{~h}$ and arginase activity in cell lysates was determined $(\mathrm{n}=$ 3). *, P $<0.05$ compared to WT. P $=0.06$ (ns) for both $0.5 \mathrm{ng} / \mathrm{ml}$ and $1 \mathrm{ng} / \mathrm{ml}$ IL-4 treatment. (B) WT (white bars) and galectin- $3^{-/-}$(black bars) BMDMs were treated with $10 \mathrm{ng} / \mathrm{ml} \mathrm{IL-4}$ or $100 \mathrm{ng} / \mathrm{ml}$ LPS for $24 \mathrm{~h}$ and total RNA extracted for real-time RT-PCR analysis. The results represent mean relative IL-4R $\alpha$ gene expression compared to $\beta$-actin $(\mathrm{n}=3) . \mathrm{P}=0.15$ (ns) and $0.28(\mathrm{~ns})$ for IL-4 and IFN $\gamma /$ LPS treatment respectively. 
C) $\quad$ Arginase/NOS2

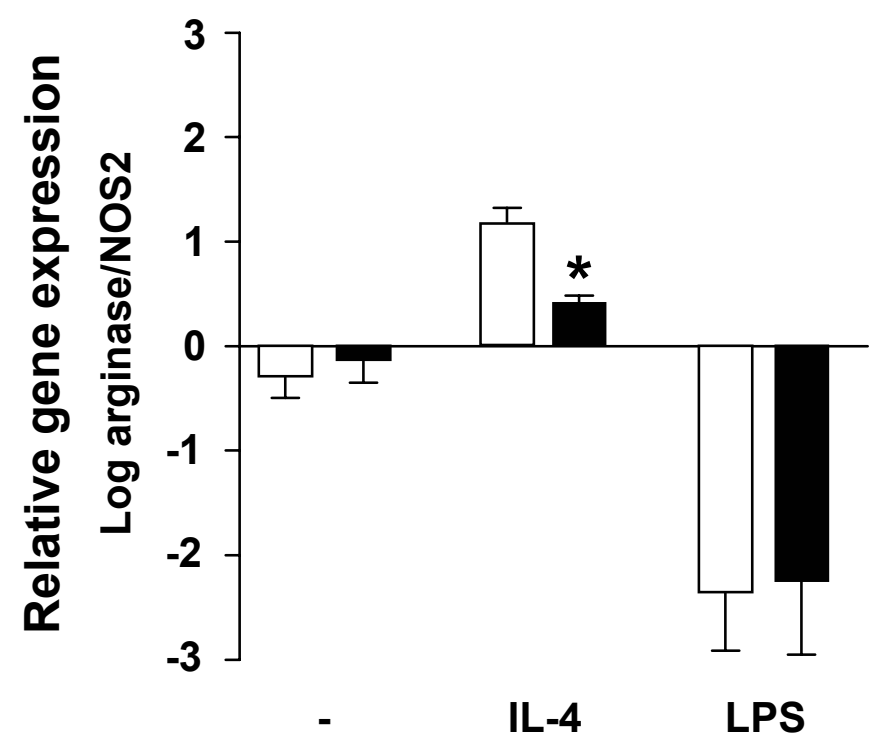

D) Mannose receptor

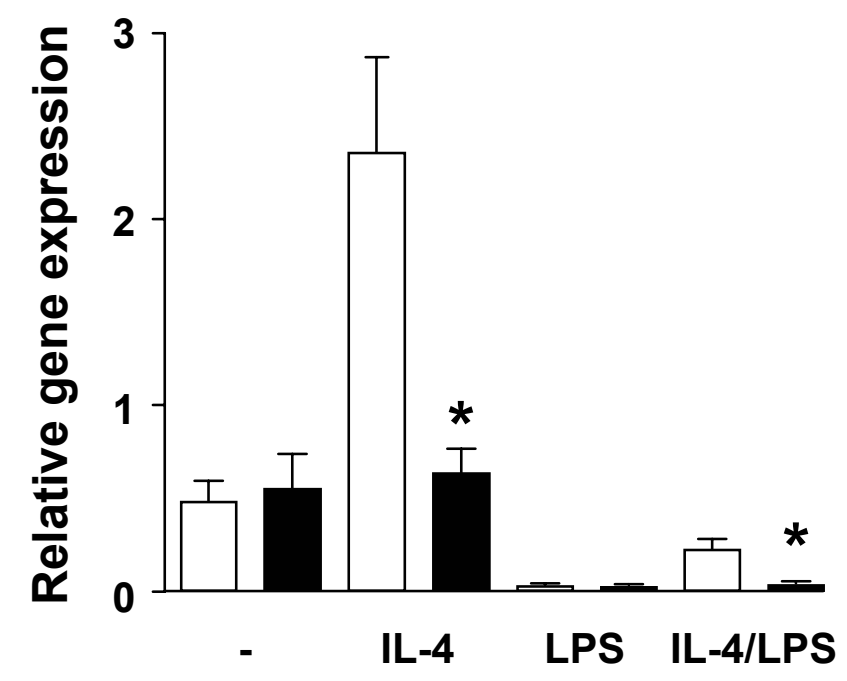

Figure 5.3 (C, D)

WT (white bars) or galectin- $3^{-/}$(black bars) BMDMs were treated with $10 \mathrm{ng} / \mathrm{ml}$ IL-4 +/- 100ng/ml LPS for 48h and total RNA extracted for real-time RT-PCR analysis. The results represent $\log [\operatorname{arginase} / \mathrm{NOS} 2$ expression ratio] $(\mathrm{C})$ and relative mannose receptor expression (D) compared to $\beta$-actin $(\mathrm{n}=3) .{ }^{*}, \mathrm{P}<0.05$ compared to WT. For LPS treatment graph (C) $\mathrm{P}=0.92$ (ns). 

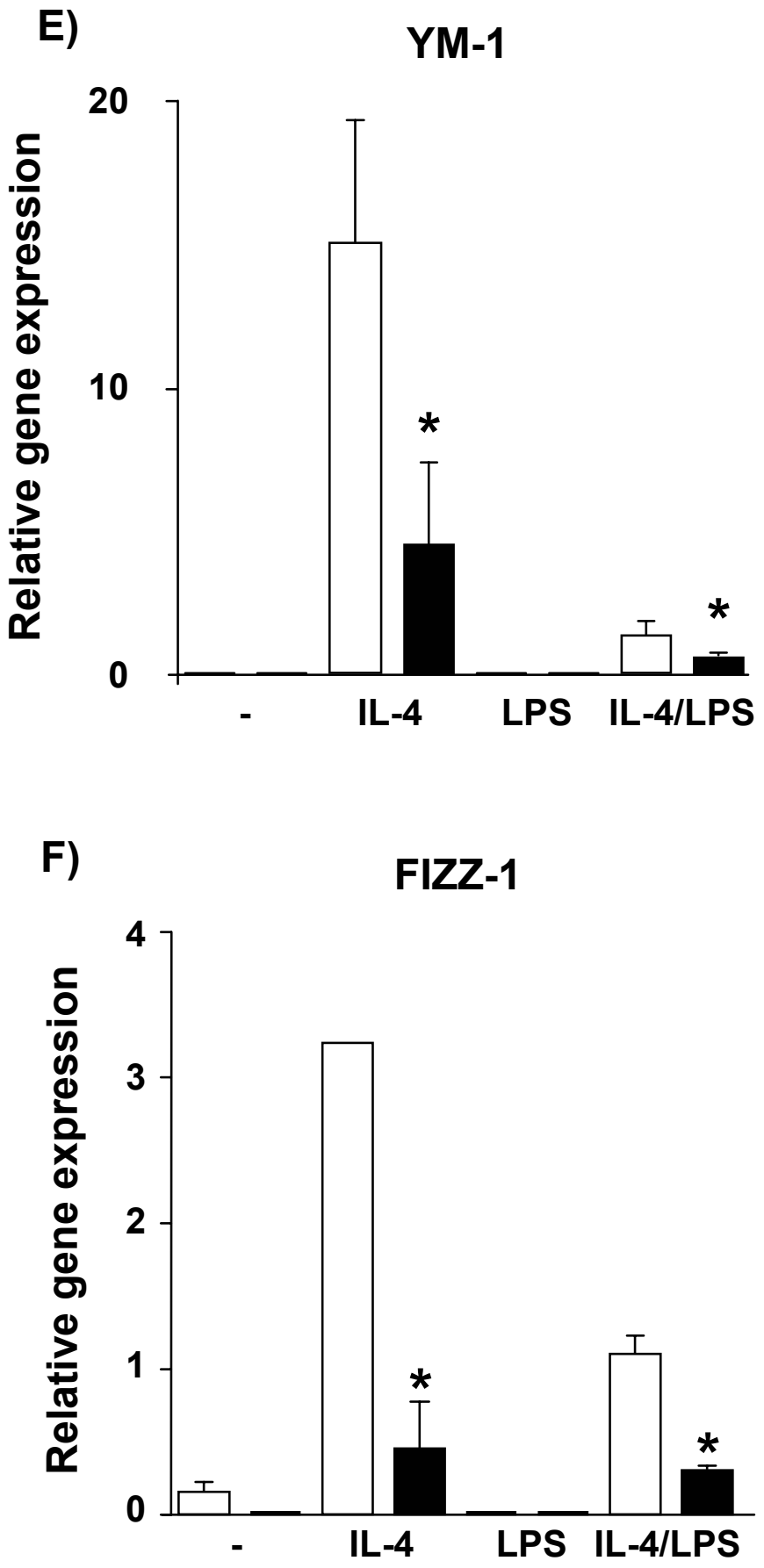

Figure 5.3 (E, F)

WT (white bars) or galectin- $3^{-/}$(black bars) BMDMs were treated with $10 \mathrm{ng} / \mathrm{ml}$ IL-4 +/- 100ng/ml LPS for 48h and total RNA extracted for real-time RT-PCR analysis. The results represent relative YM-1 (E) and FIZZ-1 (F) expression compared to $\beta$-actin $(\mathrm{n}=3)$. *, $\mathrm{P}<0.05$ compared to WT. 
LPS treatment alone or in combination with IL-4 reduced alternative activation marker expression in wild type BMDMs.

As an alternative method, in vivo differentiated mouse wild type and galectin$3^{-/-}$peritoneal and alveolar macrophages were assayed for their ability to alternatively activate. Macrophages were isolated from peritoneal and lung lavage as described in Materials and Methods and adhered to tissue culture plastic in quiescent media. Wild type and galectin- $3^{-/-}$peritoneal macrophages were stimulated with IFN $\gamma$ and LPS and nitrite and cytokine responses were compared. There was no significant difference in TNF- $\alpha$, IL-6 and nitrite release in response to IFN $\gamma /$ LPS in peritoneal macrophages isolated from wild type or galectin- $3^{-/-}$mice (figure 5.4A-C). As with BMDMs, in vivo derived peritoneal macrophages from galectin $-3^{-/-}$mice showed reduced IL-4 and IL-13 stimulated arginase activity (figure 5.4D). Western blotting bronchoalveolar lavage fluid (BAL) from galectin- $3^{-/-}$mice with anti-mouse YM-1 (kindly donated by Professor J Allen) demonstrated a decrease in expression of the alternative activation marker YM-1 compared to wild type (figure 5.4E left). In addition, wild type alveolar macrophages cultured for 24 hours in the presence of IL4 showed increased secretion of YM-1 into culture supernatants. In contrast, IL-4 did not stimulate increased YM-1 secretion from galectin- $3^{-/-}$alveolar macrophages (figure 5.4E right). Furthermore, wild type alveolar macrophages demonstrated dense intracellular YM-1 immunofluorescence, which was not observed in alveolar macrophages from galectin- $3^{-/-}$mice (figure 5.4F).

These data suggest that both in vitro and in vivo differentiated galectin- $3^{-/-}$ macrophages have a specific defect in IL-4/IL-13 stimulated alternative activation. 
Figure 5.4 - Galectin-3-- peritoneal and alveolar macrophages display normal classical activation but impaired alternative activation.

A)

\section{TNF- $\alpha$}

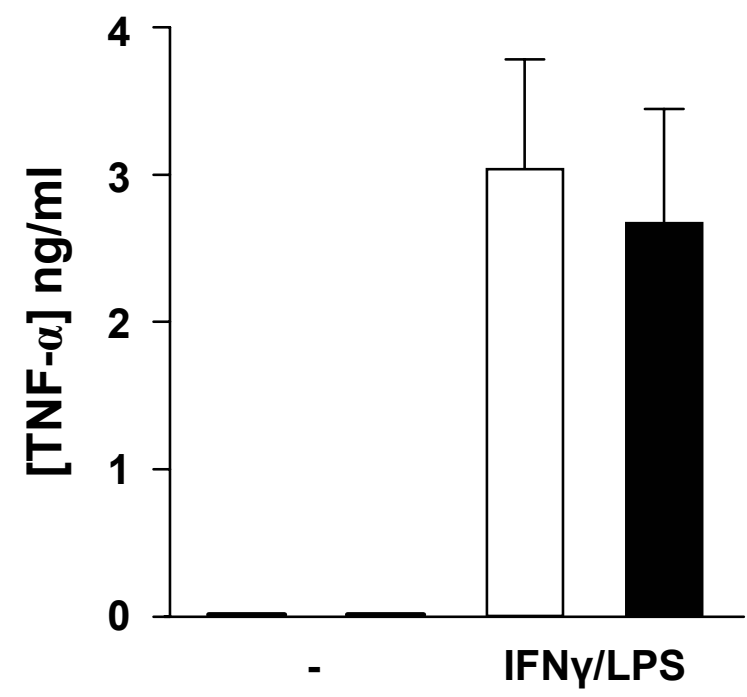

B)
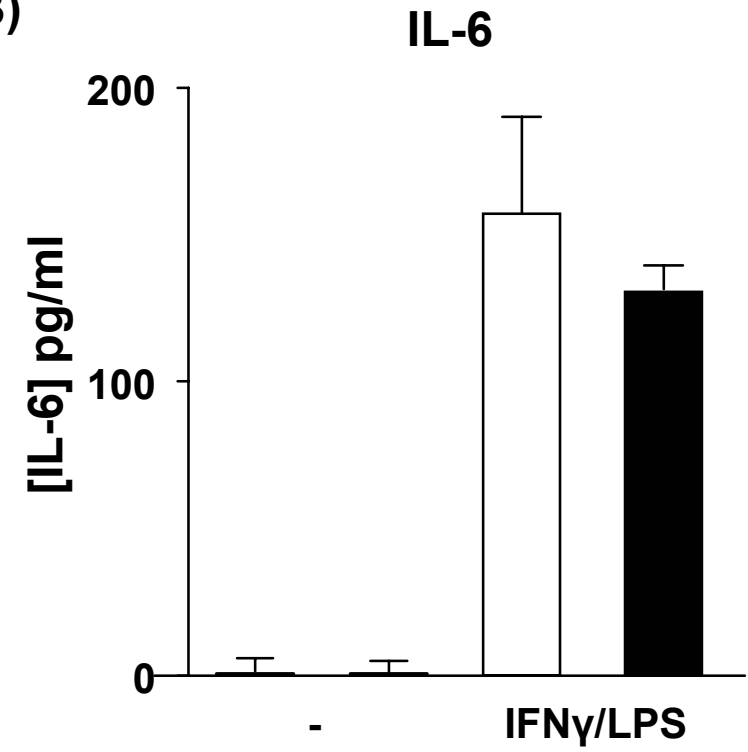

Figure 5.4 (A, B)

Peritoneal macrophages were stimulated with IFN $\gamma(100 \mathrm{U} / \mathrm{ml})$ for $48 \mathrm{~h}$ and LPS $(100 \mathrm{ng} / \mathrm{ml})$ was added for the last $24 \mathrm{~h}$. Supernatants were assayed for (A) TNF- $\alpha$ and (B) IL-6 $(\mathrm{n}=3)$. For IFN $\gamma /$ LPS treatment $\mathrm{P}=0.64(\mathrm{~ns})$ and $0.21(\mathrm{~ns})$ for (A) and (B) respectively. 
C)

Nitrite

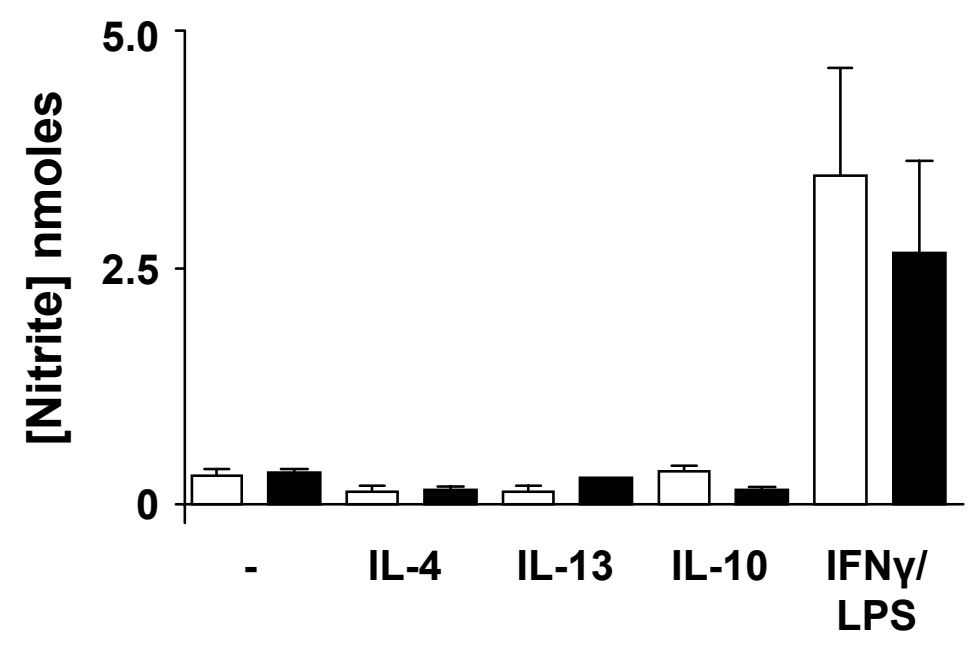

D) Arginase

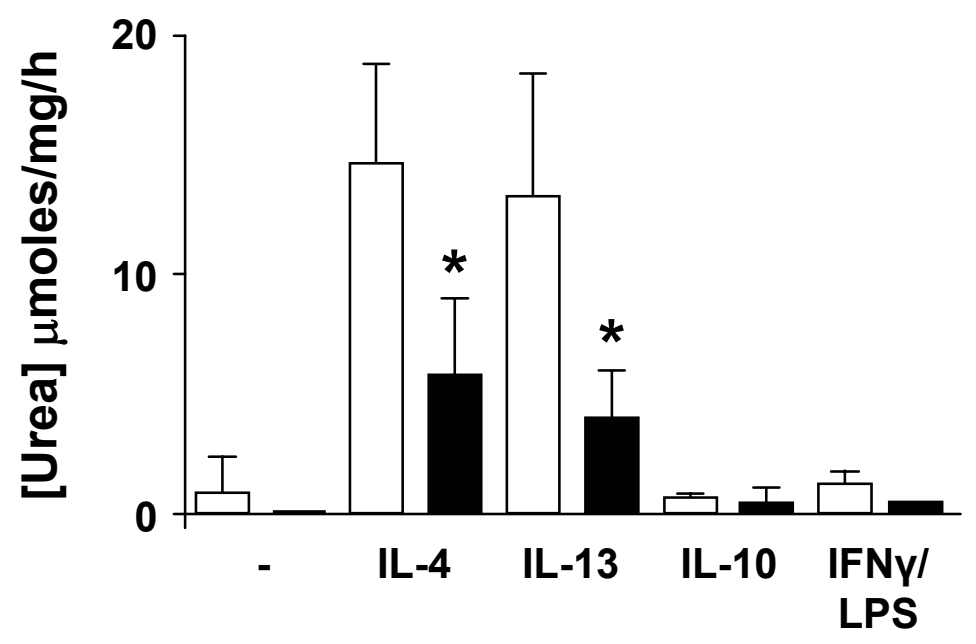

Figure 5.4 (C, D)

Peritoneal macrophages were stimulated with IL-4, IL-13, IL-10 $(10 \mathrm{ng} / \mathrm{ml})$ or IFN $\gamma(100 \mathrm{U} / \mathrm{ml})$ for $48 \mathrm{~h}$. Where indicated LPS $(100 \mathrm{ng} / \mathrm{ml})$ was added for the last 24h. Supernatants were assayed for $(\mathrm{C})$ nitrite. $\mathrm{P}=0.46$ (ns) for IFN $\gamma / \mathrm{LPS}$ treatment. (D) Cell lysates were assayed for arginase activity $(\mathrm{n}=3)$. ${ }^{*}, \mathrm{P}<0.05$ compared to WT. P $=0.51$ (ns) and 0.07 (ns) for IL-10 and IFN $\gamma /$ LPS treatment respectively. 
E)

Lavage

YM-1
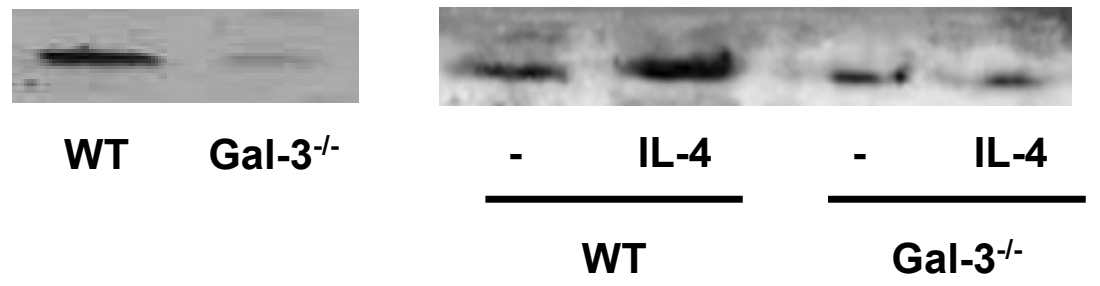

F) YM-1

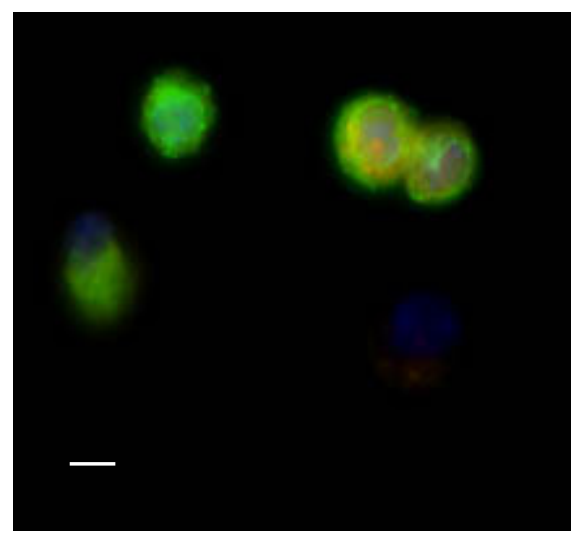

\section{Wild type}

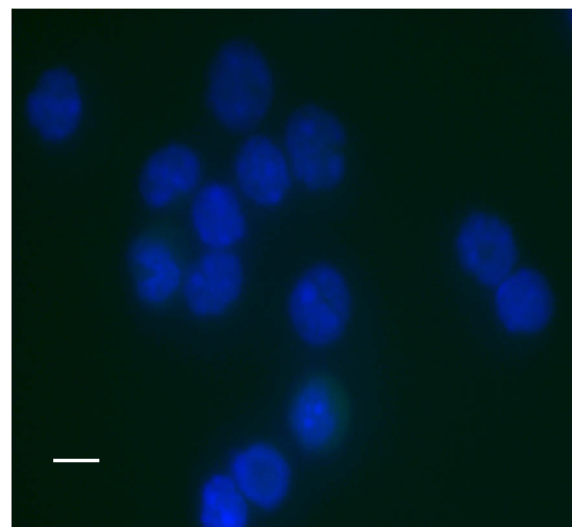

Galectin-3-/-

Figure 5.4 (E, F)

(E) $1 \mathrm{ml}$ of PBS was administered intratracheally to WT and galectin- $3^{-/-}$mice and BAL fluid was analysed for YM-1 protein expression by Western blot analysis (left). Alveolar macrophages were isolated and treated with IL-4 $(10 \mathrm{ng} / \mathrm{ml})$ for 24h and culture supernatants were analysed for YM-1 expression (right). (F) Immunofluorescence staining of YM-1 in alveolar macrophages isolated from lavage fluid of WT (top) and galectin- $3^{-/-}$(bottom) mice. (Scale bar: $5 \mu \mathrm{m}$ ). 


\subsubsection{IL-4 stimulated alternative macrophage activation is dependent on expression of galectin-3 and CD98 and activation of (phosphatidylinositol-3-kinase) PI3-K}

CD98 is highly expressed on macrophages and is a receptor for galectin-3 (Dong and Hughes, 1997). CD98 has been shown to promote "integrin-like signalling" and $\mathrm{PI} 3-\mathrm{K}$ activation causing elevation of intracellular $\mathrm{PI}(3,4,5) \mathrm{P}_{3}$ and Protein Kinase B (Akt/PKB) activation (Henderson et al., 2004; Rintoul et al., 2002). Signal transducers and activators of transcription (STATs) are involved in cytokine signal transduction. STAT-6 is an important transcription factor in IL-4 signalling. IL-4 or IL-13 bind to IL-4R $\alpha$ which causes the phosphorylation of Janus kinase (Jak) which is associated with the receptor on the cytoplasmic side of the membrane. Activated Jaks then phosphorylate IL-4R $\alpha$ at three tyrosine residues providing a docking site for STAT-6. Bound STAT-6 then becomes phosphorylated by the jaks, dimerises with a second phosphorylated STAT-6 (pSTAT-6) and translocates to the nucleus where it can modify transcriptional activity (Hebenstreit et al., 2006) (figure 5.5).

The human monocyte leukaemia cell line THP-1 was used to establish whether galectin-3-dependent alternative activation was mediated via CD98 and activation of PI3-K. Firstly, it was important to determine the expression levels of galectin-3 and CD98 in THP-1 cells. It has previously been reported that galectin-3 expression in THP-1 cells increases upon differentiation into macrophage-like cells following phorbol 12-myristate 13-acetate (PMA) treatment (Dabelic et al., 2005). Treatment of THP-1 cells with PMA, an activator of Protein Kinase C (PKC), results in the inhibition of growth at the $\mathrm{G}_{1}$-phase of the cell cycle prior to the initiation of differentiation (Traore et al., 2005). This cell cycle arrest occurs through a complex mechanism involving the modulation of expression of several cell cycle regulators, initiated by the production of reactive oxygen species (ROS). Once the cell cycle is arrested, THP-1 cells can undergo differentiation along the monocyte/macrophage pathway of development. This differentiation is inhibited by staurosporine, a potent PKC inhibitor, thus demonstrating the necessity of PKC signalling in monocyte differentiation of THP-1 cells (Barendsen et al., 1990). 
Figure 5.5 - IL-4/IL-13 signalling via the JAK/STAT signalling pathway.

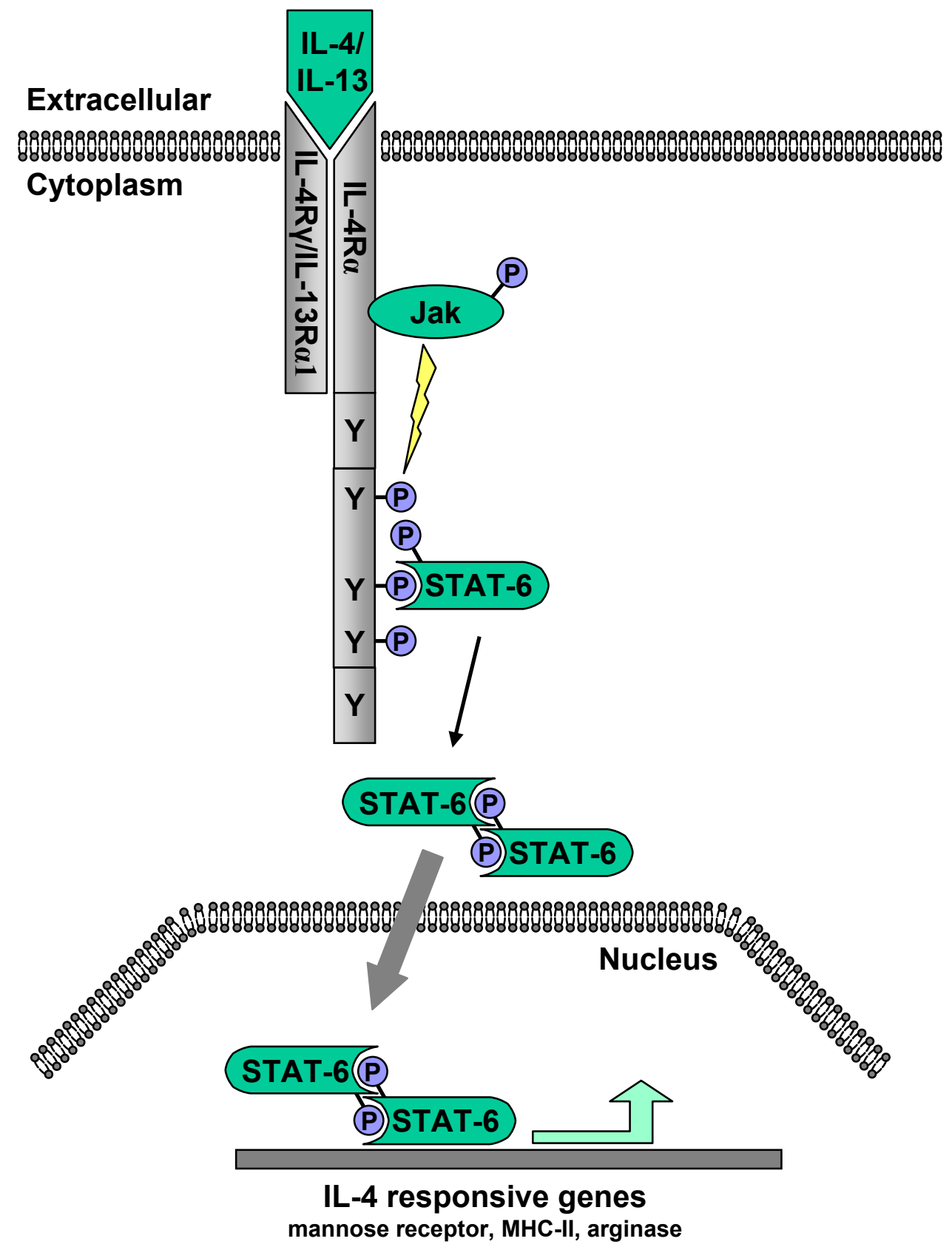

Figure 5.5

Jak/STAT signalling pathway utilised by IL-4 and IL-13. Phosphorylation of Jaks by IL-4/IL-13 binding to IL-4R $\alpha$ causes phosphorylation of IL-4R $\alpha$ providing docking sites for STAT-6. Consequent phosphorylation of STAT-6 enables its dimerisation and translocation into the nucleus where it upregulates IL-4 responsive genes. 
Differentiated THP-1 cells exhibit morphological and functional similarities to macrophages (Tsuchiya et al., 1982; Asseffa et al., 1993). The characteristic of THP-1 cells to behave more like native monocyte-derived macrophages compared to other human myeloid cell lines provides a valuable human in vitro model for studying macrophage function.

THP-1 cells were differentiated into a macrophage phenotype with 100ng/ml PMA treatment for up to 48 hours. Cell lysates were prepared at various time points and analysed by Western blotting for galectin-3 and CD98 using anti-galectin-3 (clone A3A12) and anti-human CD98 (clone C-20) antibodies respectively. This study has confirmed the finding of Dabelic et al. that galectin-3 expression increases upon THP-1 differentiation and, in addition, has demonstrated that CD98 expression also increases following PMA-treatment (figure 5.6).

To assess the role of CD98 and galectin-3 in the regulation of macrophage phenotype, a small interfering RNA (siRNA)-mediated strategy to inhibit expression was employed. Four duplexes against human CD98 and galectin-3 were designed and synthesised by Dharmacon. Duplexes were prepared as per manufacturer's instructions, transfected into differentiated THP-1 cells (48 hours post PMA treatment) using Oligofectamine and inhibition was assessed 48 hours post transfection. All four galectin-3 siRNA duplexes caused an approximate $90 \%$ knockdown of galectin-3 protein expression and CD98 siRNA duplexes 1 and 3 reduced CD98 protein expression by $>95 \%$ (figure 5.7A). CD98 protein levels remained unaffected in THP-1 cells treated with galectin-3 siRNA and vice versa. In subsequent experiments galectin-3 duplex 1 and CD98 duplex 1 were used and compared to non-targetting control duplex.

THP-1 cells transfected with control duplex or siRNA targeted to galectin-3 or CD98 were incubated for 24 hours with $10 \mathrm{ng} / \mathrm{ml} \mathrm{IL-4} \mathrm{or} 100 \mathrm{U} / \mathrm{ml} \mathrm{IFN \gamma}$ and 100ng/ml LPS. Inhibition of galectin-3 or CD98 expression blocked IL-4 stimulated increase in mannose receptor expression as judged by Western blot analysis of cell lysates with anti-human CD206 (clone 15-2) and SYBR Green real-time RT-PCR analysis of RNA extracts (figure 5.7B and C). Differentiated THP-1 cells were treated with $10 \mu \mathrm{M}$ of the PI3-K inhibitor LY294002 20 minutes prior to the addition of $10 \mathrm{ng} / \mathrm{ml} \mathrm{IL-4,} 10 \mu \mathrm{g} / \mathrm{ml} 4 \mathrm{~F} 2$ or $10 \mu \mathrm{g} / \mathrm{ml}$ recombinant human galectin-3 for 20 
minutes or 24 hours. Cell lysates were analysed by Western blotting using antipAkt/PKB (clone 14-6), anti-pSTAT-6, anti-Akt/PKB, and anti-human CD206 (clone 15-2). Cross-linking CD98 with the monoclonal antibody 4F2 or the addition of extracellular recombinant galectin-3 to THP-1 cells both stimulated PI3-K activation as measured by a sustained phosphorylation of Akt/PKB (evident at 20 minutes and maintained at 24 hours), and increased mannose receptor expression to a similar level as IL-4 (figure 5.7D). Blocking PI3-K activity with LY294002 prevented IL-4-, galectin-3-, and 4F2-mediated Akt/PKB phosphorylation and mannose receptor expression (figure 5.7D). IL-4 but not 4F2 or galectin-3 increased STAT-6 phosphorylation and this was not inhibited by LY294002 (figure 5.7D). Levels of non-phosphorylated Akt/PKB remained constant throughout all treatments.

Lysates from THP-1 cells transfected with siRNA targeted against CD98 followed by 24 hour treatment with $10 \mathrm{ng} / \mathrm{ml}$ IL-4 or $10 \mu \mathrm{g} / \mathrm{ml}$ galectin-3 were analysed by Western blotting for CD98 and pAkt/PKB. Results show that without CD98, IL-4 and galectin-3 can not stimulate Akt/PKB phosphorylation in THP-1 cells (figure 5.7E). BMDMs and primary human macrophages isolated from peripheral blood and allowed to mature for 5 days were treated for 48 hours with 10ng/ml IL-4 or IL-13 and cell supernatants were analysed for galectin-3 by Western blotting. Results show that IL-4 and IL-13 stimulated galectin-3 secretion from both macrophage types (figure 5.7F).

These data suggest that alternative activation is driven by an IL-4-stimulated galectin-3 feed back loop, which activates CD98 causing PI3-K activation. 
Figure 5.6 - Galectin-3 and CD98 expression increases in THP-1 cells following differentiation into macrophage-like cells.

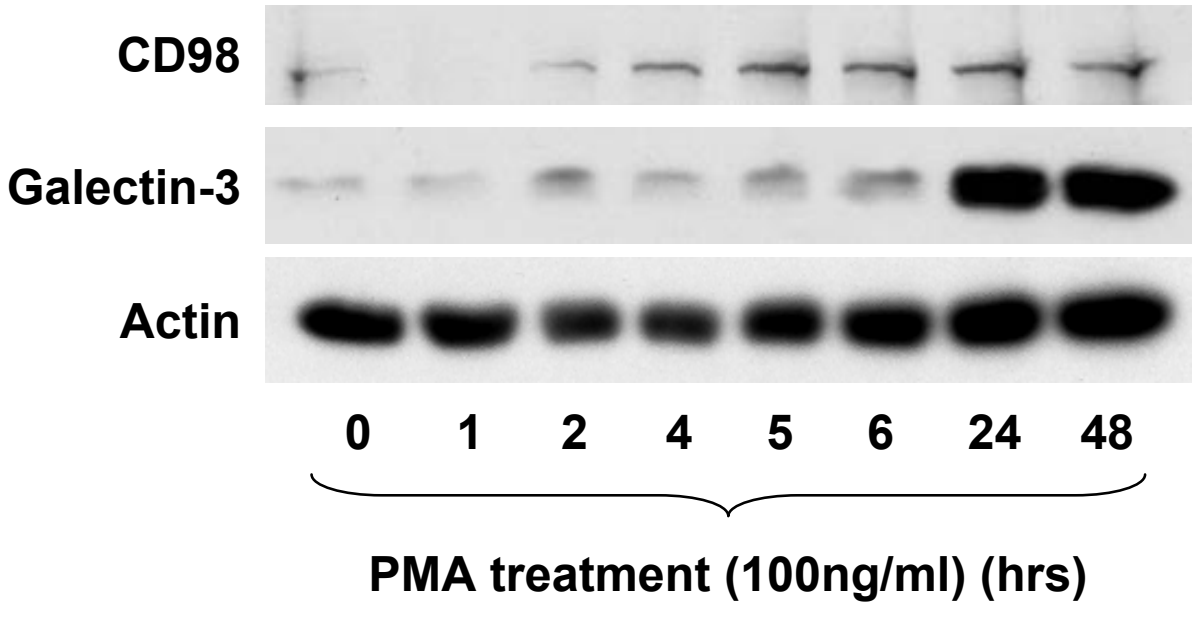

Figure 5.6

THP-1 cells were differentiated with $100 \mathrm{ng} / \mathrm{ml}$ of PMA for up to $48 \mathrm{~h}$. Cell lysates were resolved on $12 \%$ SDS-PAGE gels. Blots were probed for galectin-3, CD98 and actin expression. 
Figure 5.7 - Alternative activation of human macrophages requires galectin-3 and CD98.

A)

\section{CD98}

\section{Galectin-3}

Actin
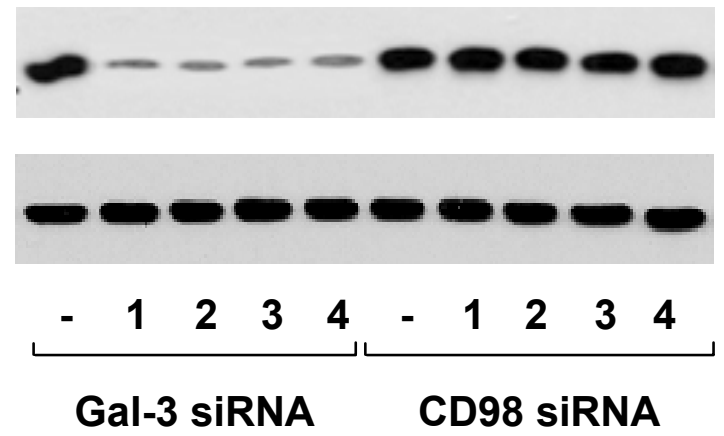

Figure 5.7 (A)

(A) THP-1 cells were differentiated with $100 \mathrm{ng} / \mathrm{ml}$ of PMA for $24 \mathrm{~h}$. Cells were transfected with $100 \mathrm{nM}$ galectin-3 or $120 \mathrm{nM}$ CD98 siRNA duplexes (1-4 as indicated) as described in Materials and Methods. 48h post-transfection cell lysates were resolved on 12\% SDS-PAGE gels. Blots were probed for galectin-3, CD98 and $\beta$-actin expression. 
B)

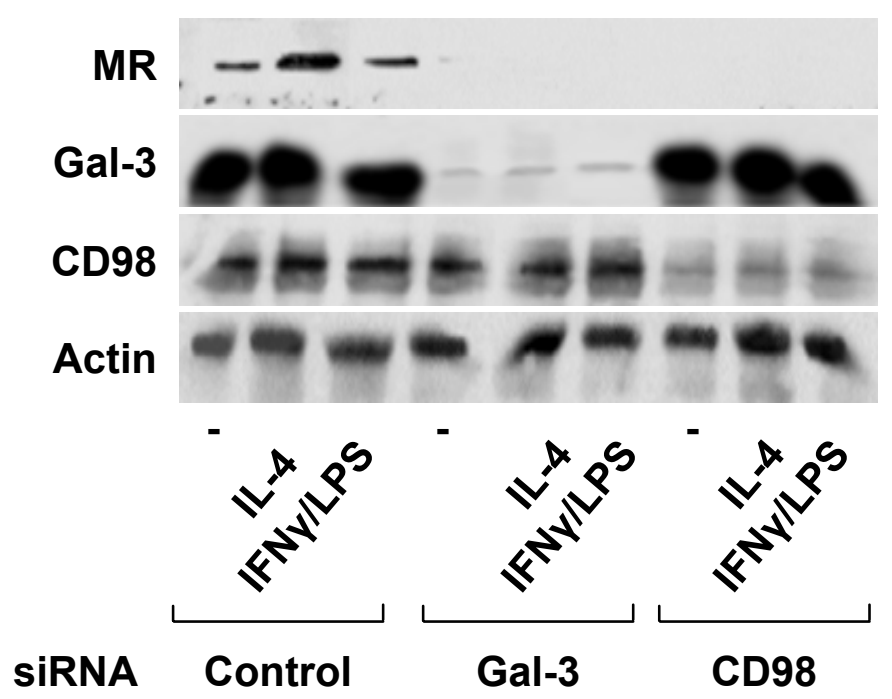

C)

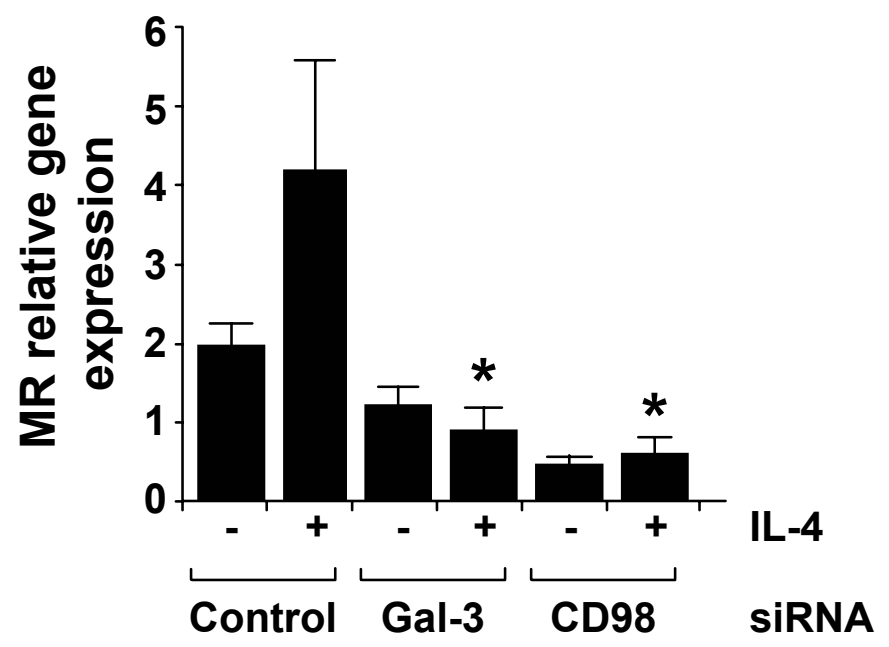

Figure $5.7(B, C)$

(B) THP-1 cells were transfected with duplex 1 of galectin-3, CD98 or control siRNA for $48 \mathrm{~h}$, followed by a $24 \mathrm{~h}$ incubation with IL-4 $(10 \mathrm{ng} / \mathrm{ml})$ or IFN $\gamma$ $(100 \mathrm{U} / \mathrm{ml}) / \mathrm{LPS}(100 \mathrm{ng} / \mathrm{ml})$. Cell lysates were resolved on $12 \%$ SDS-PAGE gels. Blots were probed for mannose receptor (MR), galectin-3, CD98 and $\beta$-actin. (C) THP-1 cells were differentiated and transfected with siRNA as in (B) followed by a $24 \mathrm{~h}$ incubation with IL-4 $(10 \mathrm{ng} / \mathrm{ml})$. Total cell RNA was extracted and mannose receptor gene expression determined by real-time RT-PCR. Results are expressed as relative gene expression compared to $\beta$-actin $(\mathrm{n}=3)$. *, $\mathrm{P}<0.05$ compared to control transfected cells. 


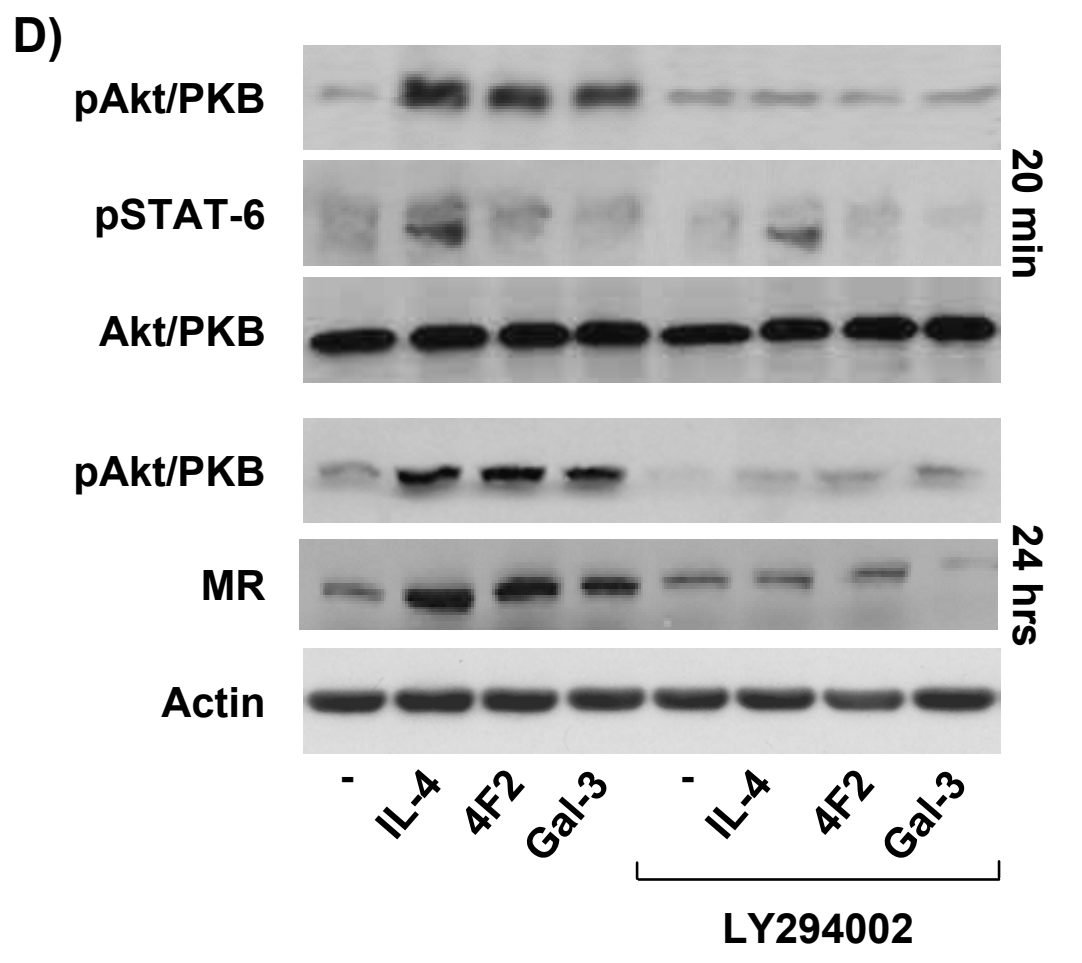

Figure 5.7 (D)

(D) Differentiated THP-1 cells were treated with $10 \mu \mathrm{M}$ LY294002 20 minutes prior to addition of $10 \mathrm{ng} / \mathrm{ml} \mathrm{IL-4,} 10 \mu \mathrm{g} / \mathrm{ml} 4 \mathrm{~F} 2$ or $10 \mu \mathrm{g} / \mathrm{ml}$ human recombinant galectin-3 for $20 \mathrm{~min}$ (top) or $24 \mathrm{~h}$ (bottom) as indicated. Western blots of cell lysates were probed for phospho-Akt/PKB (pAkt/PKB), phospho-STAT-6 (pSTAT-6), Akt/PKB, mannose receptor (MR) and $\beta$-actin as indicated. 
E)

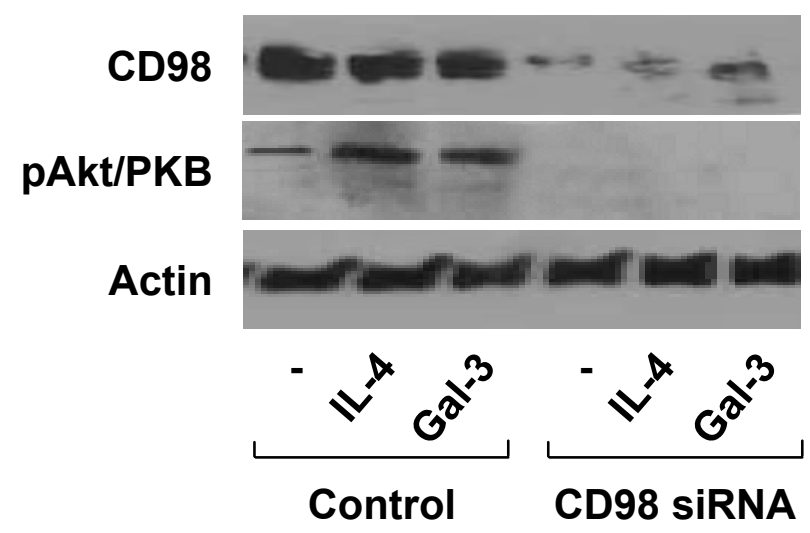

F)

Human peripheral blood macrophages Mouse BMDMs

Galectin-3
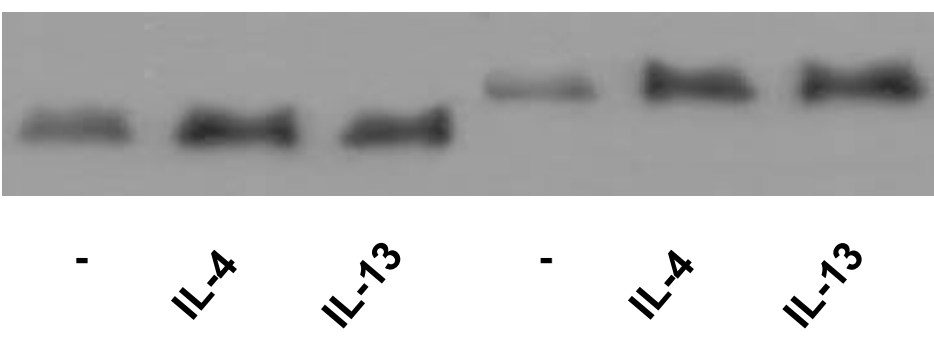

Figure 5.7 (E, F)

(E) THP-1 cells were transfected with siRNA against CD98 or control siRNA and stimulated with IL-4 or galectin-3 for $24 \mathrm{~h}$. Blots were probed for CD98, pAkt/PKB or $\beta$-actin as indicated. (F) Human peripheral blood macrophages and WT mouse BMDMs were incubated for $48 \mathrm{~h}$ with $10 \mathrm{ng} / \mathrm{ml} \mathrm{IL-4}$ or IL-13. Western blots from cell supernatants were probed for galectin-3. 


\subsubsection{Galectin- $3^{-/-}$mice show reduced expression of the alternative macrophage markers compared to wild type mice following UUO}

Previous work detailed in Chapter 4 of this thesis described galectin-3 as an important protein in the pathogenesis of fibrosis. The alternatively activated macrophage plays an important role in certain fibrotic diseases (Wynn, 2004). It was therefore hypothesised that galectin-3 may also be involved in alternative macrophage activation and that this could contribute to the progression of fibrosis.

The UUO model of renal fibrosis is a well characterised fibrosis model and demonstrates rapid infiltration of macrophages which are essential for the development of progressive fibrosis.

To assess the role of galectin-3 in alternative macrophage activation and fibrosis in vivo, RNA was extracted from obstructed kidneys 7 days after UUO, as described in Materials and Methods, and alternative macrophage activation marker expression was determined by SYBR Green real-time RT-PCR. Obstructed left kidneys from wild type mice showed a significant increase in the arginase/NOS2 ratio, mannose receptor and YM-1 expression compared to control right kidney (figure 5.8A, B and C). Obstructed kidneys from galectin- $3^{-/-}$mice showed a reduced arginase/NOS2 ratio and significantly less mannose receptor and YM-1 expression compared to wild type.

In summary, obstructed kidneys from wild type mice revealed increased levels of alternative macrophage activation compared with control kidneys demonstrated by real-time RT-PCR of whole kidney RNA extracts. Kidneys from galectin- $3^{-/-}$mice also showed increased levels of alternative macrophage activation following UUO, however this increase was significantly less than that of wild type mice. Chapter 4 of this thesis described that following UUO, galectin- $3^{-/-}$mice demonstrate decreased severity of kidney fibrosis compared to wild type mice. Together, these data suggest that galectin- $3^{-/-}$macrophages do not alternatively activate in vivo in contrast to wild type macrophages and the degree of alternative macrophage activation directly correlates with the severity of fibrosis following UUO. 
Figure 5.8 - Reduced alternatively activated macrophages in galectin-3-/- mouse kidney following UUO.

A) Arginase/NOS2 ratio

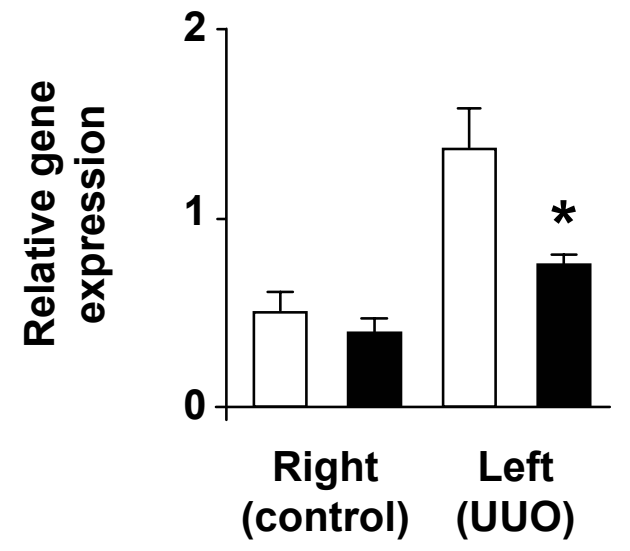

B) Mannose receptor

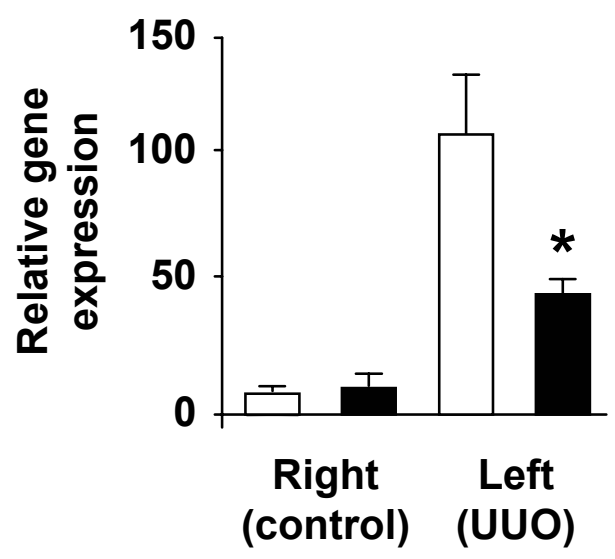

C) $\quad \mathrm{YM}-1$

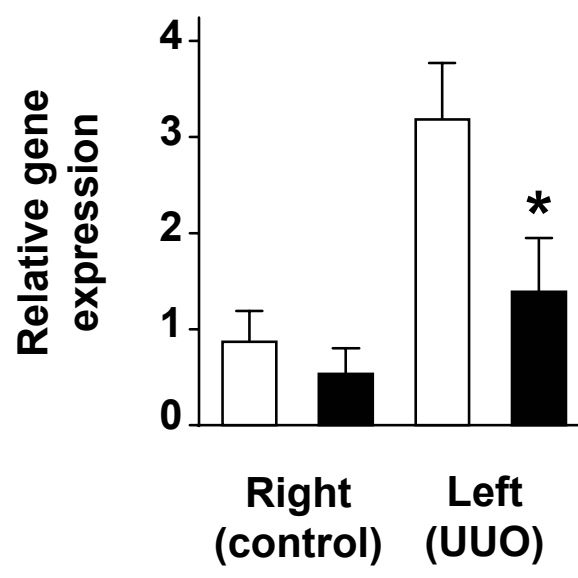

Figure 5.8 (A-C)

(A) WT (white bars) and galectin- $3^{-/-}$(black bars) mice underwent UUO of the left kidney and both left and right (control) kidneys were harvested at day 7 . RNA was extracted and arginase and NOS2 (A), mannose receptor (B) and YM-1 (C) gene expression was measured by real-time RT-PCR. The results represent the mean relative gene expression normalised to $\beta$-actin $(\mathrm{n}=3)$. ${ }^{*}, \mathrm{P}<0.05$ compared to WT left kidney. 
Figure 5.9 - Proposed galectin-3 signalling mechanism for the alternative activation of macrophages.

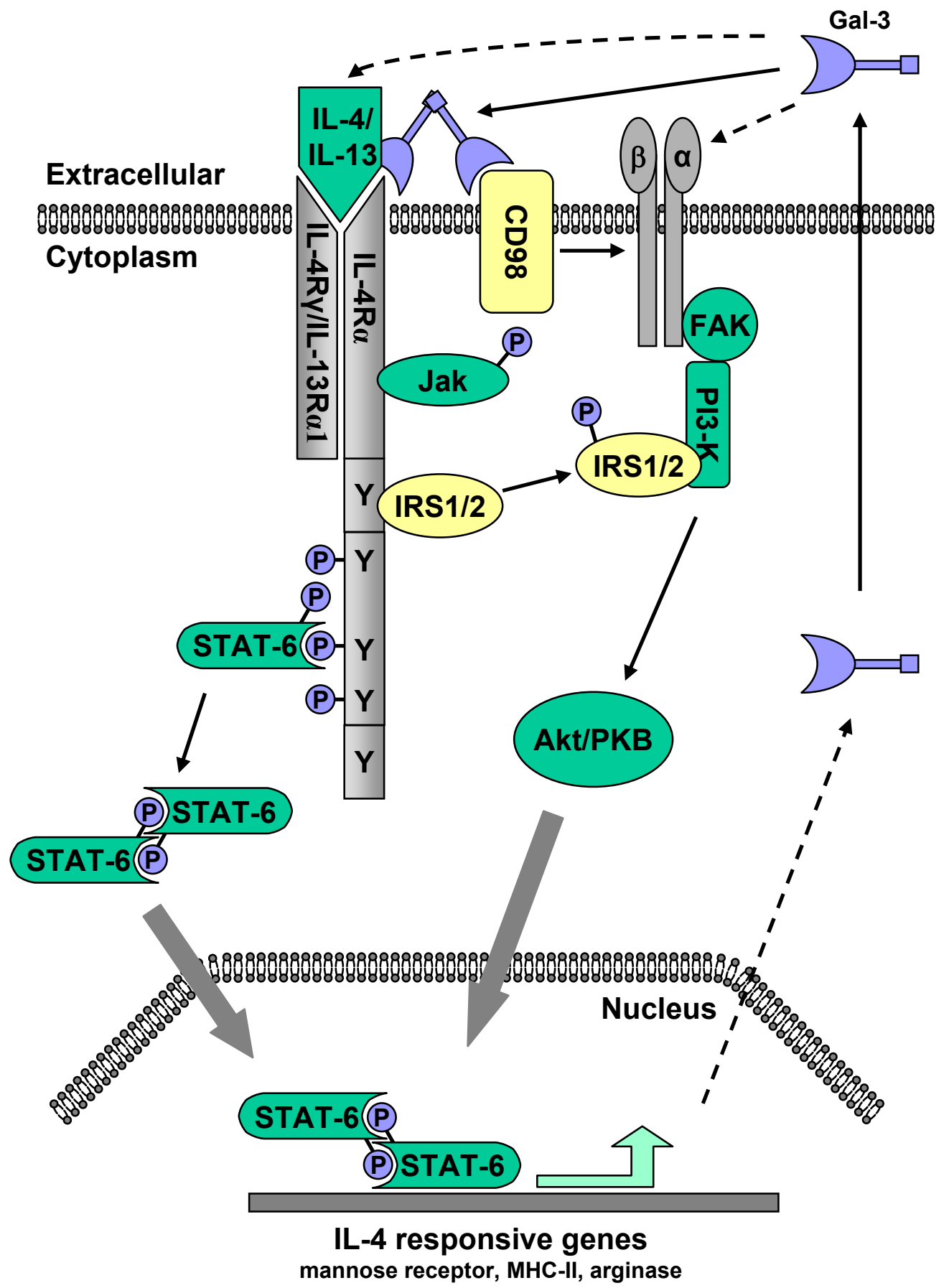

Figure 5.9

Proposed schematic showing potentiation of IL-4 mediated alternative macrophage activation by galectin-3. Dashed arrows indicate hypothesised actions or unknown mechanisms. 


\subsection{Discussion}

Previous studies demonstrated that galectin-3 plays a critical role during a fibrotic response (Henderson et al., 2006) (and Chapter 4) whereby it plays a role in myofibroblast activation. Work presented in this chapter suggests that, not only is galectin-3 important during alternative macrophage activation, but that this activation may be critical to the persistence of fibrosis. This work has also provided the mechanism through which galectin-3 causes alternative macrophage activation.

Src homology 2-containing inositol-5'-phosphate (SHIP) is an important negative regulator of the PI3-K pathway. It has been previously reported that in vitro derived BMDMs from $\mathrm{SHIP}^{-/-}$mice are M1 skewed unless exposed to TGF- $\beta$ within normal mouse plasma during differentiation whereas peritoneal and alveolar in vivo derived macrophages are M2 programmed. In vivo derived macrophages from wild type mice, however, have an M1 propensity (Rauh et al., 2005) demonstrating that macrophage phenotype can arise both during differentiation and once it's completed. In addition to the main finding, that SHIP functions in vivo to repress M2 skewing, this study cautions against extrapolating in vitro derived BMDMs to more complex in vivo systems and therefore highlights the need to utilise both in vitro and in vivo differentiated macrophages.

Initial studies were performed to investigate the role of galectin-3 in the activation of both in vitro and in vivo differentiated macrophages. In vitro derived BMDMs and in vivo differentiated peritoneal macrophages from wild type and galectin- $3^{-/}$mice were exposed to the Th1 cytokines IFN $\gamma$ and LPS or the Th2 cytokines IL-4 or IL-13 to induce a classical or an alternative macrophage phenotype respectively. Alveolar macrophages were also isolated and YM-1 expression in BAL fluid and culture supernatants from macrophages treated with IL-4 was assessed by Western blotting. YM-1 expression in alveolar macrophages was also determined by YM-1 immunofluorescence. Results suggest that both in vitro and in vivo differentiated galectin- $3^{-/-}$macrophages have a specific defect in IL-4/IL-13 stimulated alternative activation.

Once it was established that galectin-3 was involved in alternative macrophage activation, subsequent studies were carried out to determine the mechanism. The human monocytic cell line THP-1 was used to test the hypothesis 
that galectin-3-dependent alternative activation was mediated via CD98 and activation of PI3-K. Previous work demonstrated that CD98 interacts specifically with $\beta 1$ integrins and promotes "integrin-like" intracellular signalling stimulating PI3-K activity and causing elevation of intracellular $\mathrm{PI}(3,4,5) \mathrm{P}_{3}$ and $\mathrm{Akt} / \mathrm{PKB}$ activation (Henderson et al., 2004; Rintoul et al., 2002). A number of studies suggest that PI3-K activation may be the final common pathway to alternative (M2) macrophage activation. The $\mathrm{PI} 3-\mathrm{K} / \mathrm{PKB}$ pathway in macrophages negatively regulates NOS2 expression (Diaz-Guerra et al., 1999) and suppresses LPS-induced inflammation in endotoxaemic mice (Schabbauer et al., 2004). Constitutive elevation of $\mathrm{PI} 3-\mathrm{K}$ and $\mathrm{PI}(3,4,5) \mathrm{P}_{3}$ in $\mathrm{SHIP}^{-/}$mice produces $\mathrm{M} 2$ skewing (Rauh et al., 2005). These mice have high YM-1 concentrations in the lung, which may represent an exaggerated manifestation of immune tolerance and healing, which contribute to chronic lung inflammation and fibrosis.

The THP-1 cell line can be manipulated using siRNA techniques to knockdown various genes of interest. In this study, both galectin-3 and CD98 expression were successfully depleted as assessed by Western blotting. These macrophages were then treated with IL-4, and mannose receptor protein and mRNA expression was determined as a marker of alternative macrophage activation. Results verified that both galectin-3 and CD98 were required for alternative macrophage activation.

Treatment of differentiated THP-1 cells with IL-4, 4F2 and galectin-3 stimulated PI3-K activation, as measured by Akt/PKB phosphorylation. Blocking PI3-K activation, with the inhibitor LY294002, inhibited IL-4-, galectin-3- and 4F2stimulated alternative macrophage activation, assessed by mannose receptor expression. Furthermore, IL-4 but not 4F2 or galectin-3 increased STAT-6 phosphorylation and this was not inhibited by LY294002. CD98 is required for both IL-4 and galectin-3-induced PI3-K activation, demonstrated by an inhibition of Akt/PKB phosphorylation in THP-1 cells treated with CD98 siRNA. IL-4 caused stimulation of galectin-3 expression in both mature human peripheral blood macrophages and mouse BMDMs.

The following additional work carried out by other members of this laboratory has expanded the volume of data regarding the role of galectin- 3 during 
alternative macrophage activation. A high affinity inhibitor of galectin-3, Bis-(3deoxy-3-(3-methoxybenzamido)-B-D-galactopyranosyl)-sulfane, blocked alternative macrophage activation suggesting that the carbohydrate recognition domain of galectin-3 is responsible for these effects and supports an extracellular mechanism of action. STAT-6 siRNA in THP1 cells blocked IL-4 and galectin-3-stimulated alternative macrophage activation, suggesting that while STAT-6 activation is not sufficient to drive alternative macrophage activation, some basal activity is required to enable sustained PI3-K activation to drive alternative macrophage activation. This is consistent with the findings that STAT6 $6^{--}$mice have significantly less collagen I kidney deposition than wild type mice following UUO

These results suggest that IL-4 stimulates a galectin-3 feed back loop causing sustained PI3-K activation and that this is the key mechanism required for activation of an alternative macrophage phenotype. Figure 5.9 illustrates the proposed schematic showing potentiation of IL-4 mediated alternative macrophage activation by galectin-3. IL-4 binding to the IL-4R $\alpha$ chain recruits the IL-13R $\alpha 1$ chain. Activation of the JAK/STAT pathway results in recruitment of insulin receptor substrate-1 (IRS-1) to the first cytoplasmic tyrosine residue (Y1) which binds to the p85 subunit of PI3-K resulting in the activation of the p110 catalytic subunit and phosphorylation of $\mathrm{PIP}_{2}$ to $\mathrm{PIP}_{3}$ and activation of downstream signalling intermediates such as Akt/PKB. Phosphorylation of the second, third or fourth cytoplasmic tyrosine residues results in recruitment of STAT- 6 which, when tyrosine is phosphorylated, dimerizes and migrates to the nucleus where it binds to consensus sequences found within the promoters of IL-4 regulated genes resulting in up regulation of genes associated with alternative activation. CD98 when activated by galectin-3 or crosslinked with 4F2 results in integrin-dependent activation of PI3-K and Akt/PKB activation. The schematic proposes that galectin-3 potentiates IL-4 mediated alternative activation directly by activating CD98 and increasing $\mathrm{PIP}_{3}$ levels thereby raising the threshold for alternative activation. Galectin-3 may also potentiate IL-4 signalling by binding to N-Glycan residues expressed on the IL-4R and by homotypic aggregation may stabilise a high affinity complex of IL-4R/CD98 and $\beta 1$-integrin on the cell surface causing sustained activation of pathways leading 
to alternative activation. It is proposed that IL-4 stimulates galectin-3 expression and release leading to further potentiation of IL-4 mediated signalling.

Alternative macrophage activation has also been shown in this study to be important for the initiation and development of renal fibrosis following UUO. Renal fibrosis had long been recognised as a prominent feature of diseased kidneys. However the pathologic mechanisms during the evolution of fibrotic changes in the kidney, including the role of infiltrating macrophages in each stage of the fibrotic process, have not yet been fully illucidated. Macrophages are involved in all stages of the inflammatory process including fibrosis, tissue repair and healing (Nagaoka et al., 2000; Teder et al., 2002). Evidence in mouse models of liver fibrosis, glomerulonephritis and renal fibrosis, suggest that macrophages also down-regulate injury and facilitate repair. During progressive inflammatory injury, macrophage depletion results in amelioration of fibrosis. By contrast, depletion during recovery results in a failure of resolution with persistence of cellular and matrix components of the fibrotic response (Duffield et al., 2005a). Thus macrophages play distinct roles in injury and repair highlighting that macrophages may be both pathogenic and beneficial depending on the timing and injury.

Further work carried out by this laboratory utilised the CD11b-diptheria toxin receptor (DTR) mouse (Duffield et al., 2005b; Duffield et al., 2005a; Cailhier et al., 2005 ) to confirm the role of macrophages in tubulointerstitial scarring. These mice express the human DTR under the control of the CD11b promoter. This system relies on the fact that the mouse DT receptor binds DT poorly compared with the human molecule. Thus, transgenic expression of the human DTR confers sensitivity to DT and permits specific ablation of monocytes and macrophages in vivo when DT is injected (Duffield et al., 2005a; Cailhier et al., 2005). Results showed that infiltrating macrophages drive myofibroblast activation and resultant interstitial fibrosis as indicated by reduced $\alpha$-SMA and collagen expression evident in the obstructed kidney of macrophage depleted mice. Moreover, adoptive transfer of wild type macrophages into galectin- $3^{-/-}$mice drove the activation of interstitial myofibroblasts as indicated by significantly increased expression of $\alpha$-SMA in contrast to galectin- $3^{-/-}$macrophages which did not. These results show that galectin- 
$3^{-/-}$fibroblasts in the kidney have the potential to transdifferentiate into myofibroblasts when activated by wild type macrophages.

The dual function of macrophages may be due to their ability to alter phenotype under different microenvironments and represent a mechanism where they can be inflammatory or reparative depending on context. Galectin- $3^{-/-}$mice may develop reduced fibrotic damage due to their inability to adopt a "fibrotic" macrophage phenotype. Moreover, this work suggests that increased galectin-3 expression may be a feature of the alternative macrophage phenotype and this increased expression is driven by IL-4. Increased galectin-3 expression and release by macrophages may also contribute to fibrosis by directly activating myofibroblasts.

Previous work highlighted in chapter 4 of this thesis demonstrated that galectin- $3^{-/}$mice are protected from renal fibrosis following UUO despite developing a macrophage infiltrate comparable to wild-type mice. In vitro data demonstrated a deficiency of galectin $-3^{-/-}$macrophages to alternatively activate, therefore the activation status of renal macrophages following UUO was determined by real-time RT-PCR analysis of total RNA extracts from kidneys. Studying macrophage phenotype in situ was carried out as it is assumed that macrophages isolated from kidneys would rapidly alter their phenotype when cultured in vitro. Findings from this study revealed that the levels of alternative macrophage activation markers were significantly reduced in obstructed kidneys from galectin- $3^{-/-}$mice compared to wild type mice. Further work carried out by this laboratory demonstrated colocalisation of YM-1 and mannose receptor expression with macrophages in tissue sections from obstructed wild type kidneys. Results demonstrated tubular expression of YM-1 in galectin- $3^{-/-}$kidneys but no significant co-expression on macrophages and absent mannose receptor expression. This study revealed that macrophages in obstructed kidneys from galectin- $3^{-/-}$mice are significantly less alternatively activated than those from wild type mice.

This study provides insight into the mechanisms regulating alternative macrophage activation and may provide more clearly defined therapeutic targets in a wide range of human diseases. Targeting the galectin-3/CD98/PI3-K pathway with specific inhibitors such as Bis-(3-deoxy-3-(3-methoxybenzamido)- $\beta$-D- 
galactopyranosyl)-sulfane may represent a novel therapeutic target for manipulating macrophage phenotype in the treatment of cancer, chronic inflammation and fibrosis. 


\section{Chapter 6}

\section{Galectin-3 and pneumonia}

\subsection{Introduction}

Streptococcus pneumoniae $(S . p n)$ is the leading cause of community acquired pneumonia (CAP) worldwide with resulting high mortality rates (Brown and Lerner, 1998). Limitations of conventional therapies and emerging drug resistance amongst strains of $S . p n$ to antibiotics, such as penicillin (Feldman, 2004) and vancomycin (Novak et al., 1999), necessitates the continued study into the mechanisms involved in the pathogenesis of pneumococcal disease and the host immune defence against pneumococcal invasion.

Previous studies have demonstrated the ability of galectin-3 to manipulate immune cell function. Galectin-3 plays a role in cell adhesion (Inohara and Raz, 1995; Kuwabara and Liu, 1996; Sato et al., 2002), activation (Liu et al., 1995; Yamaoka et al., 1995; Karlsson et al., 1998; Fernandez et al., 2005; Frigeri et al., 1993; Hsu et al., 1996) and phagocytosis of apoptotic thymocytes by macrophages (Sano et al., 2003) and IgG-opsonised erythrocytes by macrophages (Sano et al., 2003) and neutrophils (Fernandez et al., 2005). I have also shown that galectin-3 plays a role in skewing macrophages to the pro-fibrotic M2 phenotype (Chapter 5). The absence of galectin-3 can alter the outcome of certain disease states including fibrosis (examined Chapter 4) and, as shown in this chapter, of acute pneumococcal infection.

Alveolar macrophages, recruited macrophages and neutrophils play a key role in the clearance and killing of invading pathogens. In the lung, resident alveolar macrophages are the first line of cellular defence and play a phagocytic role during the early stages of infection. Interaction of these cells with pneumococcus provokes an inflammatory response inducing recruitment of the more efficient polymorphonuclear leukocytes (PMNs or neutrophils) and increased concentrations of serum opsonins at the site of infection. Amongst the earliest leukocytes recruited to the site of infection are neutrophils. Neutrophils play a critical role in the host 
immune defence against infection through their ability to phagocytose and kill bacteria.

PMN migrate into an inflammatory site from the vascular space. The selectin family of adhesion molecules tethers leukocytes to the endothelium via weak cellcell interactions. This contact slows the circulating neutrophils down allowing them to roll on the endothelium. The rolling process initiates stronger adhesion mediated by leukocyte integrins. In the case of Escherichia coli (E. coli) infection, this tight adhesion is mediated by $\beta_{2}$ integrins (Hogg and Doerschuk, 1995). Conversely, neutrophil extravasation following pneumococcal pneumonia is selectin-independent (Mizgerd et al., 1996) and is observed in $\beta_{2}$ integrin knockout mice (Mizgerd et al., 1997; Mizgerd et al., 1999). Therefore the mechanisms involved in the host response to different pathogens are distinct. Work carried out in the study detailed in this chapter was prompted by these findings and also from those of Sato et al. who demonstrated that galectin-3 accumulates in the alveolar space of mice infected with $S$. $p n$ and that this accumulation of galectin-3 correlates with the onset of neutrophil extravasation. However, accumulation of galectin-3 was not observed when neutrophil extravasation was induced by an E. coli infection (Sato et al., 2002). Furthermore it was demonstrated that galectin-3 is released by alveolar macrophages upon incubation with $S$. pn membrane fraction. It was therefore proposed that galectin-3 acts as a neutrophil adhesion molecule during pneumococcal pneumonia. In addition, lipopolysaccharide (LPS) expressed on E. coli has been shown to down regulate galectin-3 expression (Liu et al., 1995; Sato and Hughes, 1994). In these studies, although a role for galectin-3 in response to pneumococcal pneumonia was implicated, it was not shown how galectin-3 may function to orchestrate the inflammatory response to infection or whether galectin-3 deficiency would result in animals succumbing to infection.

Activation of neutrophils and phagocytosis of microbes results in the increased consumption of molecular oxygen by professional phagocytes. This process, catalysed by NADPH-oxidase, results in the production of superoxide $\left(\mathrm{O}_{2}{ }^{-}\right)$ and hydrogen peroxide $\left(\mathrm{H}_{2} \mathrm{O}_{2}\right)$. The bactericidal activity of neutrophils is mediated, in part, through the generation of these reactive oxygen species (ROS) during the normal host defence against infectious disease. There are numerous techniques used 
to measure ROS production. This study utilises two of these well established techniques, the reduction of cytochrome $\mathrm{C}$ by ROS and a flow-based assay measuring dihydrorhodamine 123 (DHR) fluorescence. Cytochrome C molecules are reduced by ROS resulting in a change in absorbance which can be measured spectrophotometrically. This method measures the $\mathrm{O}_{2}^{-}$metabolite (Dahlgren and Karlsson, 1999). DHR is a fluorescent dye which is specifically responsive to the $\mathrm{H}_{2} \mathrm{O}_{2}$ metabolite (Walrand et al., 2003). DHR enters the membrane of the cell where it is oxidised by ROS to rhodamine 123 thus emitting a bright fluorescent signal which can be measured by flow cytometry.

Phagocytosis of apoptotic neutrophils by macrophages is a key process in the resolution of inflammation. Clearance of potentially toxic apoptotic neutrophils by macrophages results in the release of anti-inflammatory and reparative cytokines such as Transforming Growth Factor- $\beta 1$ (TGF- $\beta 1$ ). These steps help to limit the degree of tissue injury.

In this chapter, a direct comparison of the effect of galectin-3 expression on the development of pneumonic infection induced by the gram-positive $S$. pn and the gram-negative E. coli will address the hypothesis that upregulation of galectin-3 expression by $S$. pn activates neutrophils, promotes bacterial clearance and plays a role in the resolution of inflammation. These data provide evidence for galectin-3 based mechanisms as a potential therapeutic strategy for treating pneumonia.

\subsection{Results}

\subsubsection{In vivo studies}

To determine the effects of galectin-3 deficiency on the outcome of a pneumococcal and E. coli pneumonia infection, wild type and galectin- $3^{-/-}$mice were inoculated with $1 \times 10^{5}$ colony forming units (CFU) S. pn or $1 \times 10^{7}$ CFU E. coli intratracheally (I.T.). These doses were calculated based on previous dose-finding pilot studies carried out by Dr N. Henderson and were chosen to produce acute inflammatory injury without significant mortality after 15 hours in wild type mice.

15 hours post inoculation mice were sacrificed and the severity of infection was ascertained by the following means: 
1. Total cell counts and neutrophil and macrophage counts in bronchoalveolar lavage (BAL) fluid were calculated to determine the amount of cell recruitment to the site of infection.

2. Pulmonary microvascular leakage, as determined by BAL protein concentration, was used to assess the extent of lung injury.

3. Degree of septicaemia was measured by aseptically plating blood taken from each animal on a $5 \%$ sheep blood agar plate for S. pn or Luria-Bertani (LB) agar plate for E. coli and incubating overnight at $37^{\circ} \mathrm{C}$. The following morning, culture plates housing bacterial colonies indicated a positive result of septicaemia in the corresponding animal.

4. Efficiency of bacterial clearance was assessed by calculating the numbers of bacteria present in BAL fluid and lung homogenate. Samples were serially diluted, inoculated on 5\% sheep blood agar plates for $S$. $p n$ or LB agar plates for E. coli overnight at $37^{\circ} \mathrm{C}$ and bacterial counts of $\mathrm{CFU} / \mathrm{ml}$ were determined.

\subsubsection{Galectin-3 plays a role in the clearance of $S$. pn from the lungs and protects the lung from damage}

In vivo, galectin- $3^{-/-}$mice demonstrated a clearance defect of $S$. pn with increased septicaemia and a greater extent of lung damage. Protein concentration was significantly higher in BAL fluid from galectin- $3^{-/-}$mice compared to wild type mice (figure 6.1A) indicating a greater degree of lung injury. 8 out of $8(100 \%)$ culture plates inoculated with galectin- $3^{-/-}$blood produced colonies following $S$. $p n$ infection compared to just 2 out of 7 (28.6\%) of wild type mice (figure 6.1B). Culture plates of lung homogenate from galectin- $3^{-/-}$mice produced approximately 450 -fold greater bacterial load than culture plates inoculated with wild type lung homogenate $\left(8.6 \times 10^{8} \mathrm{CFU} / \mathrm{ml}\right.$ in galectin- $3^{-/-}$compared to $1.9 \times 10^{6} \mathrm{CFU} / \mathrm{ml}$ in wild type) (figure 6.1C) indicating a clearance defect of galectin- $3^{-/-}$mice against $S$. pn. However bacterial counts in BAL were not significantly different between the two groups (figure 6.1D). There were reduced total cell numbers and neutrophil numbers in BAL fluid from galectin- $3^{-/-}$mice (figure $6.1 \mathrm{E}$ and $\mathrm{F}$ ), however, the numbers of macrophages retrieved was similar between the two groups (figure 6.1G) . 
Figure 6.1 - Galectin-3 plays a role in the clearance of an acute pneumococcal infection.

A)

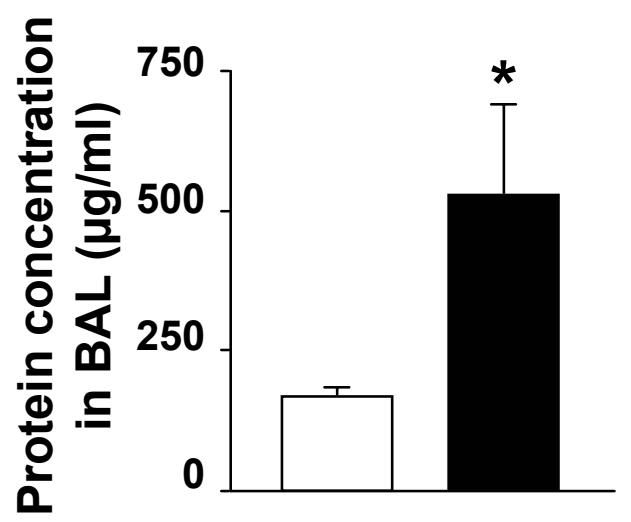

B)

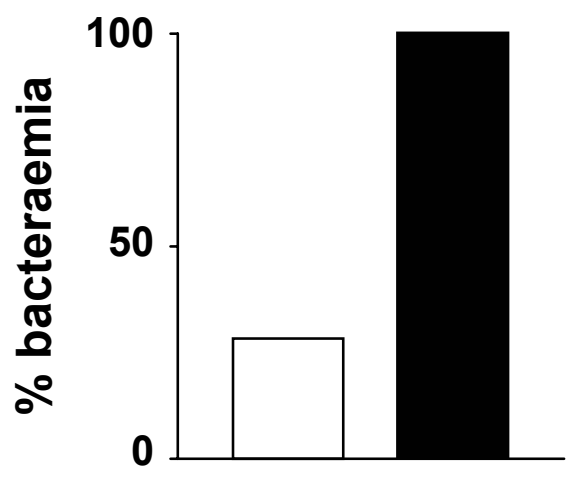

C)

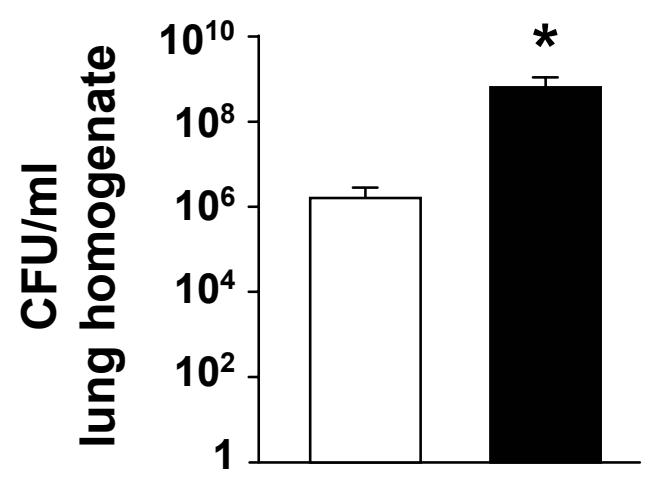

Figure 6.1 (A-C)

WT (white bars) and galectin- $3^{-/}$(black bars) mice were inoculated with $1 \times 10^{5}$ CFU Streptococcus pneumoniae (S. pn) for $15 \mathrm{~h}\left(\mathrm{n}=7 \mathrm{WT}, \mathrm{n}=8\right.$ galectin- $\left.3^{-/-}\right)$. (A) Protein concentration in BAL fluid from WT and galectin- $3^{-/-}$mice. $*, \mathrm{P}<$ 0.05 compared to WT. (B) Blood samples from galectin- $3^{-/}$and WT mice were plated on blood agar plates, the percentage of plates showing the presence of bacterial colonies was taken and expressed as \% bacteraemia. (C) Lungs from galectin- $3^{-/-}$and WT mice were homogenised, serially diluted, plated on blood agar plates and colonies counted. *, P $<0.05$ compared to WT. 
D)

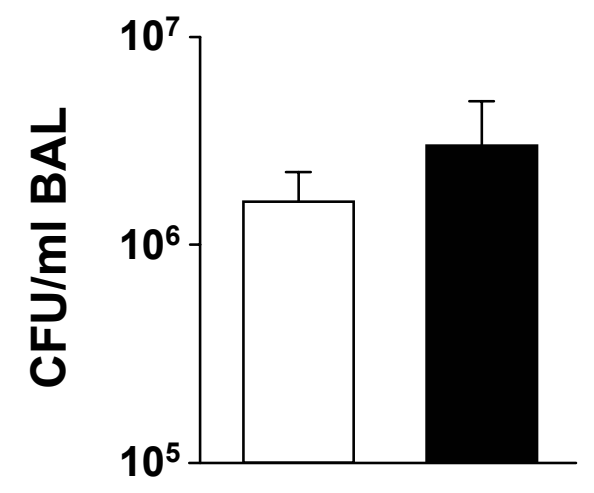

F)

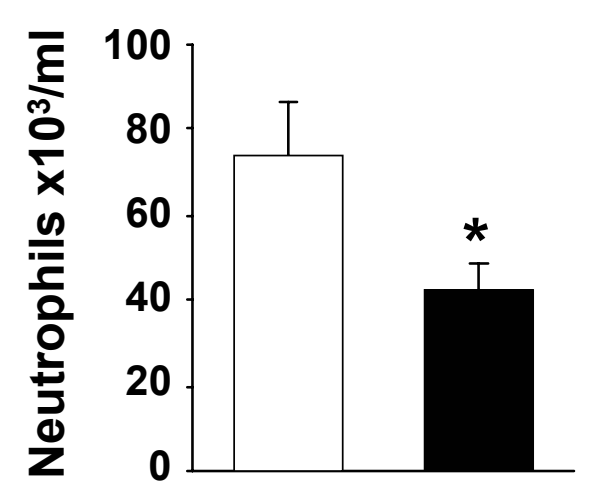

E)

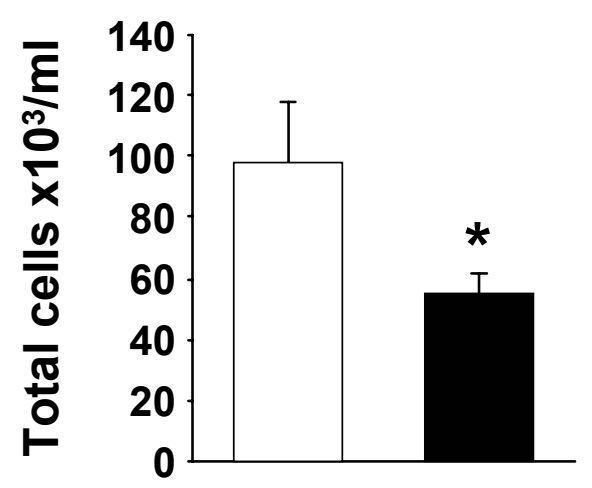

G)

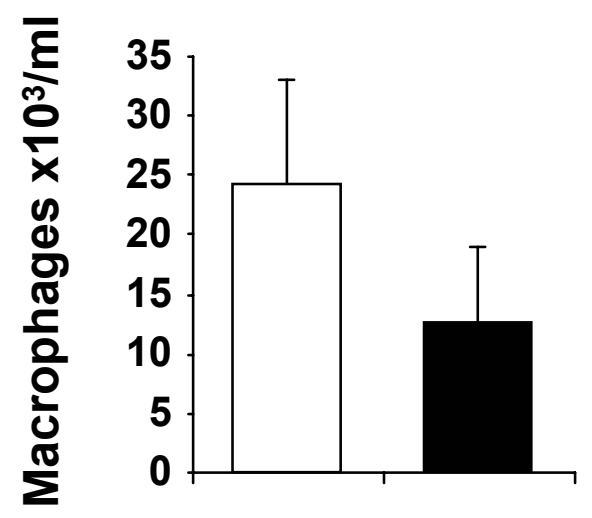

Figure 6.1 (D-G)

(D) Bacterial counts in WT (white bars) and galectin-3/-- (black bars) BAL fluid. $\mathrm{P}=0.43$ (ns). Total cell counts (E), neutrophil $(\mathrm{F})$ and macrophage $(\mathrm{G})$ counts in lavage fluid of galectin- $3^{-/-}$and WT mice inoculated with $S . p n$ for $15 \mathrm{~h} .{ }^{*}, \mathrm{P}<$ 0.05 compared to WT. $\mathrm{P}=0.25$ (ns) for $(\mathrm{G})$. 
These data verify galectin-3 as a critical molecule in the clearance of a pneumococcal pneumonia infection.

\subsubsection{Galectin-3 does not play a significant role in the clearance of $E$. coli pneumonia}

Galectin- $3^{-/-}$and wild type mice demonstrated equal bacteraemia following $E$. coli infection with 5 out of 11 (45\%) culture plates inoculated with galectin- $3^{-/-}$blood and 5 out of $10(50 \%)$ culture plates inoculated with wild type blood producing colonies (figure 6.2A). This was in stark contrast to the response to $S$. $p n$ infection (figure 6.1B). Culture plates of lung homogenate from galectin- $3^{-/-}$mice produced more bacterial colonies than wild type culture plates, although this result did not reach statistical significance (figure 6.2B). Bacterial counts in BAL were not significantly different between the two groups (figure 6.2C). Neutrophil and macrophage recruitment was comparable between wild type and galectin- $3^{-/-}$mice treated with E. coli (figure 6.2D and E) and wild type and galectin- $3^{-/-}$lung weights were similar (figure 6.2F).

The striking difference in disease outcome of a pneumococcal pneumonia infection in galectin- $3^{-/-}$mice compared to wild type is not as prominent with an $E$. coli pneumonia infection. This indicates that galectin-3 may still play a role in bacterial clearance of the latter infection but is not as critical for survival, however further experiments need to be performed to verify this result. 
Figure 6.2 - Galectin-3 does not play a significant role in the clearance of an acute $E$. coli pneumonia infection.

A)

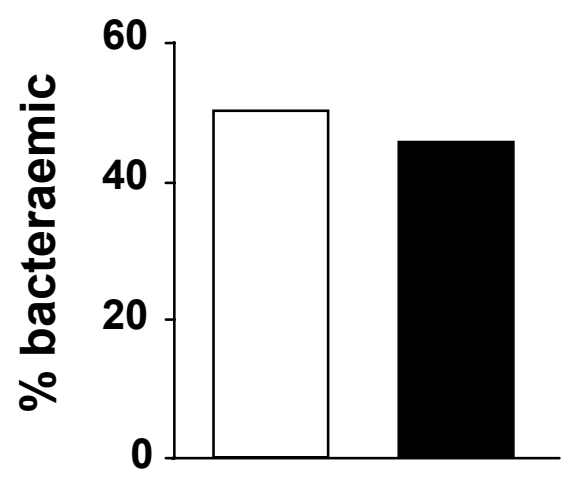

B)

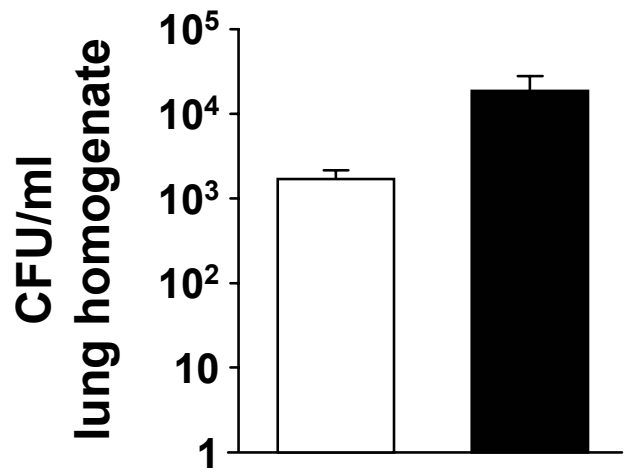

\section{C)}

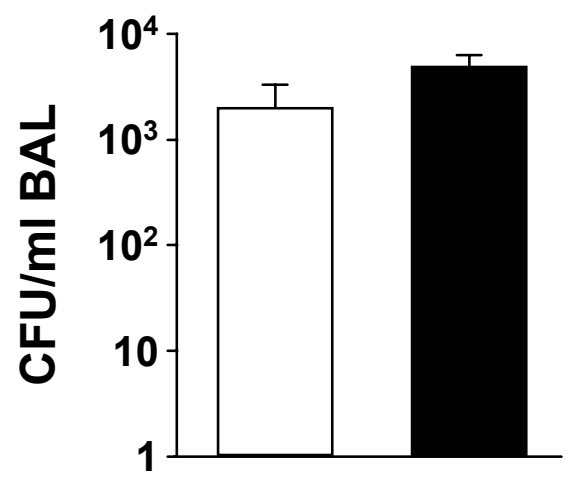

Figure 6.2 (A-C)

WT (white bars) and galectin- $3^{--}$(black bars) mice were inoculated with $1 \times 10^{7}$ CFU Escherichia coli (E. coli) for $15 \mathrm{~h}\left(\mathrm{n}=10 \mathrm{WT}, \mathrm{n}=11\right.$ galectin- $\left.3^{-/-}\right)$. (A) Blood samples from galectin- $3^{-/}$and WT mice were plated on LB agar culture plates, the percentage of plates showing the presence of bacterial colonies was taken and expressed as \% bacteraemia. (B) Lungs from galectin- $3^{-/-}$and WT mice were homogenised, serially diluted, plated on LB agar culture plates and colonies counted. $\mathrm{P}=0.09$ (ns). (C) Bacterial counts in lavage fluid from WT and galectin- $3^{-/-}$mice. $\mathrm{P}=0.09(\mathrm{~ns})$. 


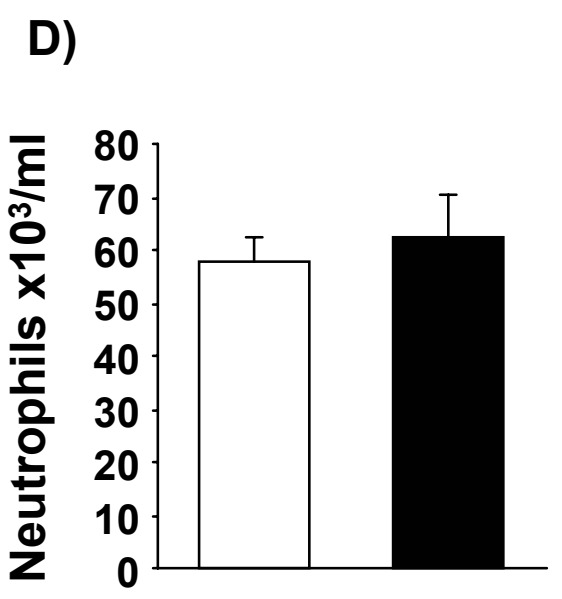

E)

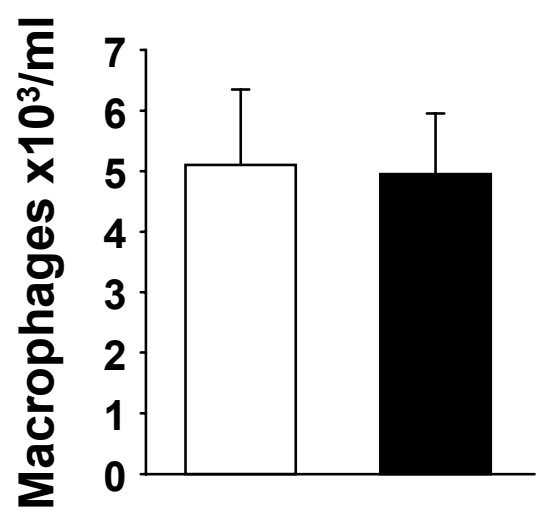

F)

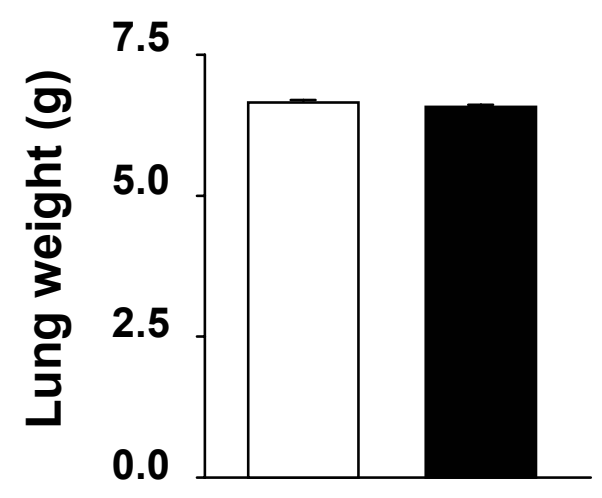

Figure 6.2 (D-F)

Neutrophil (D) and macrophage (E) counts per ml of lavage fluid of WT (white bars) or galectin- $3^{-/}$(black bars) mice inoculated with $E$. coli for $15 \mathrm{~h} . \mathrm{P}=0.63$ (ns) and 0.49 (ns) for (D) and (E) respectively. (F) WT and galectin-3 $3^{-/-}$lung weights following inoculation with $E$. coli. $\mathrm{P}=0.16$ (ns). 


\subsubsection{Galectin-3 is bactericidal against $S$. $p n$ at high concentrations}

Galectin-3 has recently been described to have antimicrobial activity towards the pathogenic fungus Candida albicans (Kohatsu et al., 2006). It was therefore important to investigate whether galectin-3 had a direct antimicrobial effect on $S$. $p n$. The same strain of $S$. $p n$ used in the in vivo experiment was grown to an $\mathrm{OD}_{600}=0.1$. The $S$. pn were diluted 1:100 and $75 \mu 1$ of this bacterial reaction mix was incubated with $75 \mu 1$ of varying concentrations of galectin-3 or galectin-3 denatured for $10 \mathrm{~min}$ at $95^{\circ} \mathrm{C}(6.25,12.5,25,50$ or $100 \mu \mathrm{g} / \mathrm{ml})$ for 2 hours in vitro as described in Materials and Methods. Serially diluted samples of untreated bacteria were plated out at $t=0$ and at $\mathrm{t}=2$ hours after shaking (control). A sample of $S$. $p n$ was incubated with ampicillin $(20 \mu \mathrm{g} / \mathrm{ml})$ as a positive antibiotic control. 2 hours post-incubation, samples were serially diluted and blood agar plates were inoculated with each reaction of $S$. pn. Colonies were counted the following day and the numbers of viable bacteria were determined. Addition of $100 \mu \mathrm{g} / \mathrm{ml}$ galectin-3 to $S$. $p n$ significantly inhibited growth with the lower concentrations of galectin-3 and denatured galectin-3 showing no effect on growth (figure 6.3). This result could partially explain my in vivo findings that galectin- $3^{-/-}$mice demonstrate reduced bacterial clearance.

\subsubsection{Galectin- ${ }^{-/-}$macrophages produce less nitrite, compared to wild type, in response to both S. pn and E. coli in vitro}

Alveolar macrophages and recruited macrophages and neutrophils play a key role in the clearance and killing of invading pathogens. In vivo studies indicated a clearance defect of bacteria in galectin- $3^{-/-}$mice. This work led on to the hypothesis that the presence of galectin-3 secreted by resident and recruited macrophages in response to a pneumococcal pneumonia infection activates recruited leukocytes in the lung and subsequently promotes phagocytosis and bacterial clearance. Further in vitro studies were performed to investigate the mechanisms whereby galectin-3 regulates macrophage and neutrophil function. 
Figure 6.3 - Antimicrobial activity of galectin-3 to S. pn.

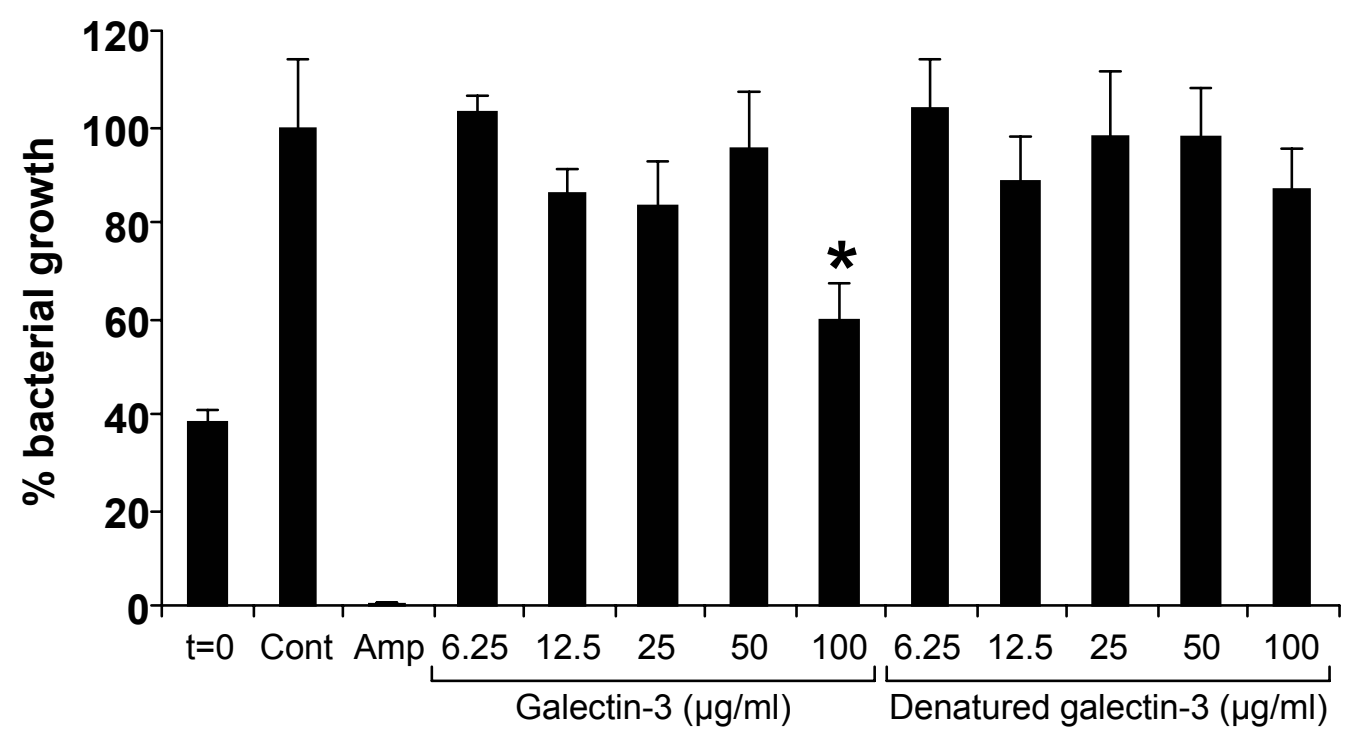

Figure 6.3

Percentage bacterial growth of $S$. $p n$ following incubation with varying concentrations of galectin-3, denatured galectin-3 or ampicillin (Amp 20 $\mu \mathrm{g} / \mathrm{ml}$ ) for 2 hours $(\mathrm{n}=3){ }^{*}, \mathrm{P}<0.05$ compared to untreated control and $100 \mu \mathrm{g} / \mathrm{ml}$ denatured galectin-3. $\mathrm{P}>0.05$ for all other treatments compared to untreated control or equivalent denatured control. 
Nitric oxide (NO) release from macrophages in response to bacteria helps to restrain bacterial growth. NO helps to control a variety of infections including parasitic (Liew et al., 1991; Petray et al., 1995), viral (Bi and Reiss, 1995) and fungal infections (Alspaugh and Granger, 1991). Furthermore Tsai et al. demonstrated that NO plays a critical role in the antibacterial defence against Klebsiella pneumoniae infection (Tsai et al., 1997).

NO production from wild type and galectin- $3^{-/-}$macrophages in response to $S$. $p n$ and E. coli was examined to test the hypothesis that galectin- $3^{-/-}$macrophages may be less efficient at killing $S$. $p n$. Chapter 5 of this thesis demonstrated no difference in NO production from wild type and galectin- $3^{-/-}$mice following treatment with the Th1 cytokines Interferon- $\gamma$ (IFN $\gamma$ )/LPS. However, nitrite release in response to bacteria may be affected by a lack of galectin-3.

Wild type and galectin- $3^{-/-}$bone marrow derived macrophages (BMDMs) treated with IFN $\gamma /$ LPS produced equal concentration of nitrite (figure 6.4A). This result confirms the finding in Chapter 5 that wild type and galectin- $3^{-/}$macrophages can classically activate to equal extents. Galectin- $3^{-/-}$BMDM produced significantly less nitrite in response to both $S$. $p n$ and $E$. coli when compared to their wild type counterparts (figure 6.4B) suggesting a defect in bacterial killing. In addition, galectin- $3^{-/}$peritoneal macrophages incubated with opsonised $S$. pn also demonstrated reduced levels of nitrite production compared to wild type peritoneal macrophages (figure 6.4C).

To test that the reduction in nitrite production was not due to greater cell death of the galectin- $3^{-/-}$BMDM in response to bacteria, a trypan blue viability assay was performed as described in Materials and Methods. This assay demonstrated no difference in cell death between wild type and galectin- $3^{-/-}$BMDM after incubation with either S. pn or E. coli (figure 6.4D).

Reduced nitrite production from galectin $-3^{-/-}$macrophages in response to both S. $p n$ and E. coli, could therefore explain the clearance defect observed in vivo. 
Figure 6.4 - Galectin-3-- macrophages release less nitrite in response to both $S$. $p n$ and $E$. coli but not in response to IFNy/LPS.

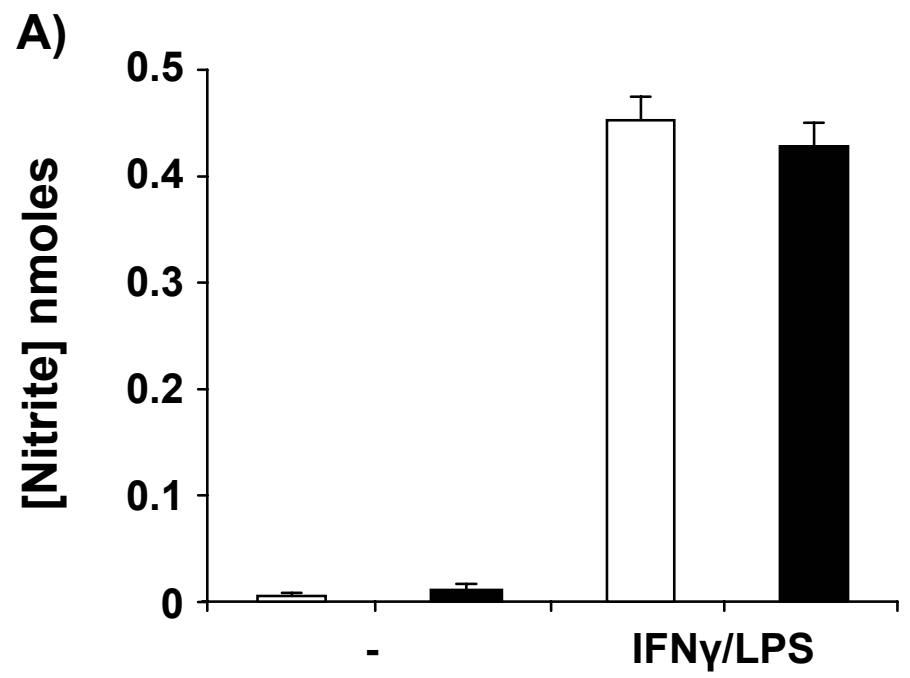

B)

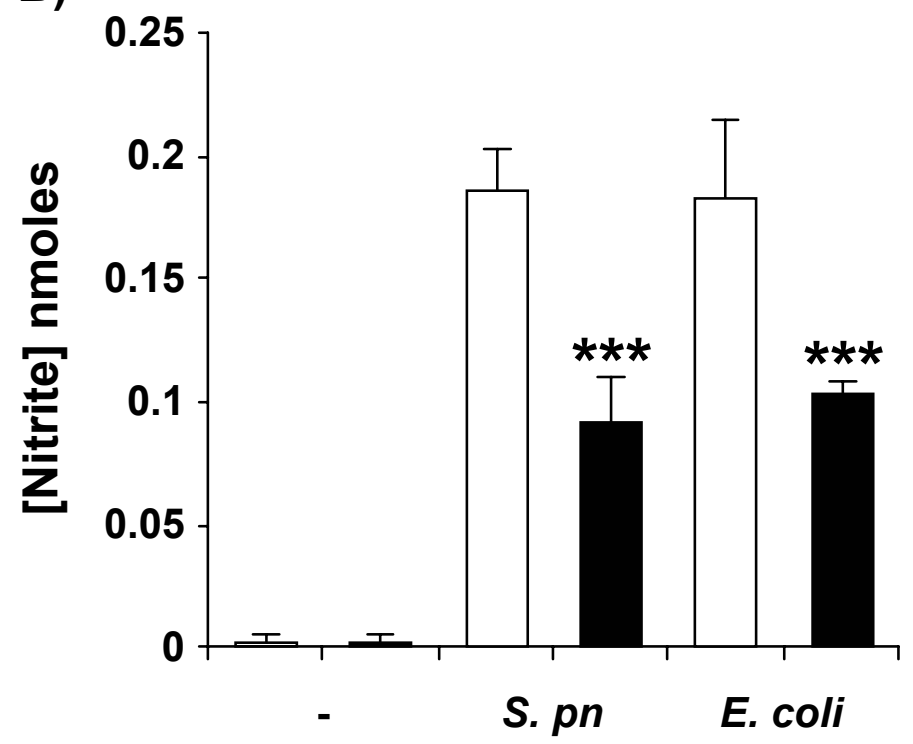

Figure 6.4 (A, B)

BMDM prepared from WT (white bars) and galectin- $3^{--}$(black bars) mice were incubated with (A) IFN $\gamma(100 \mathrm{U} / \mathrm{ml}) / \mathrm{LPS}(100 \mathrm{ng} / \mathrm{ml})$ or (B) a $2: 1$ ratio of opsonised $S$. pn or E. coli to BMDMs for 24h. Supernatants were reacted in a 1:1 ratio with Greiss Reagent and absorbance at $\lambda 560 \mathrm{~nm}$ was measured. Nitrite concentration was calculated using the gradient equation from the standard curve $(\mathrm{n}=5)$. ${ }^{* * *}, \mathrm{P}<0.0001$ compared to WT. (A) $\mathrm{P}=0.14$ (ns) for IFN $\gamma / \mathrm{LPS}$ treatment. 
C)

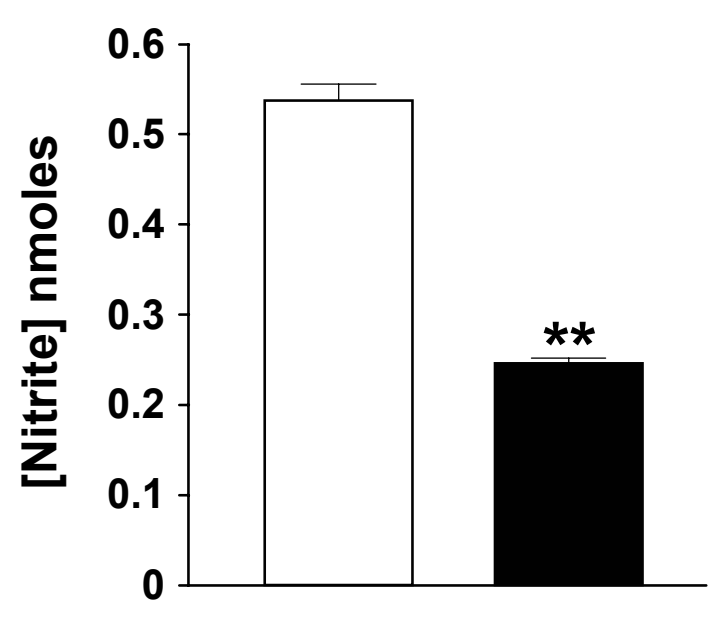

D)

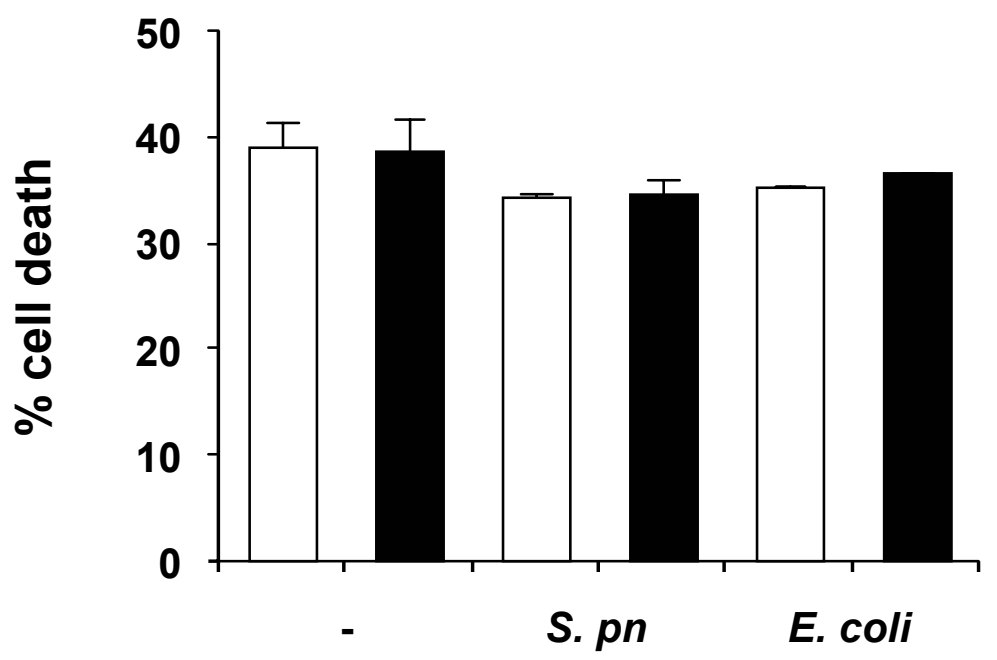

Figure 6.4 (C, D)

(C) Nitrite release from WT (white bars) and galectin- $3^{-/-}$(black bars) peritoneal macrophages incubated with a $2: 1$ ratio of $S . p n$ for $24 \mathrm{~h}(\mathrm{n}=3)$. **, $\mathrm{P}<0.01$ compared to WT. (D) Percentage cell death, measured by trypan blue viability assay described in Materials and Methods, of WT (white bars) and galectin- $3^{-/-}$ (black bars) BMDM after 24 hour treatment with 2:1 opsonised S. pn or E. coli (n $=3)$. 


\subsubsection{Galectin-3 $^{-/-}$macrophages demonstrate similar levels of phagocytosis to wild type macrophages}

Previous studies have shown that galectin- $3^{-/-}$macrophages exhibit reduced phagocytosis of IgG opsonised erythrocytes and apoptotic thymocytes (Sano et al., 2003). However, to date there has been no study investigating the role of galectin-3 during phagocytosis of bacteria. To test the hypothesis that the absence of galectin-3 may result in macrophages being less efficient at phagocytosing bacteria in a pneumonic infection, BMDM and alveolar macrophages were incubated with FITClabelled heat-killed $S$. $p n$ and E. coli in vitro and degree of phagocytosis assessed using both fluorescent-activated cell sorting (FACS) analysis and confocal microscopy.

Galectin-3 $3^{-/-}$and wild type BMDM were incubated with a 10:1 ratio of opsonised FITC-labelled $S$. $p n$ and E. coli for up to 1 hour, washed, removed from the culture plate and analysed using FACS analysis. Macrophages were gated on according to forward and side scatter properties (R1) (figure 6.5A). Figure 6.5B shows forward scatter versus FL1 properties of untreated BMDMs and BMDMs incubated with $S$. pn or E. coli as described above. Percentage events in upper right quadrant indicates macrophages that have phagocytosed FITC-labelled bacteria. The results from 3 independent experiments were pooled and percentage BMDMs containing FITC-labelled bacteria (compared to wild type, 60 minutes) was calculated and revealed to be similar for both galectin- $3^{-/}$and wild type BMDMs (figure $6.5 \mathrm{C}$ and $\mathrm{D}$ ). To confirm this finding, confocal analysis of wild type and galectin-3 ${ }^{-/-}$BMDMs incubated with $S . p n$ and E. coli, and alveolar macrophages incubated with $S$. pn, was performed. Macrophages were seeded on coverslips, incubated with opsonised bacteria for 1 hour, washed, and incubated with rhodamine phalloidin to stain the actin cytoskeleton and 4',6-diamidino-2-phenylindole (DAPI) to stain the nucleus. Coverslips were mounted on slides and analysed using a Leica SP5 confocal microscope and subsequently processed using Adobe Photoshop. 
Figure 6.5 - Galectin-3 is not required for effective phagocytosis of $S$. pn or E. coli by BMDM or alveolar macrophages.

A)

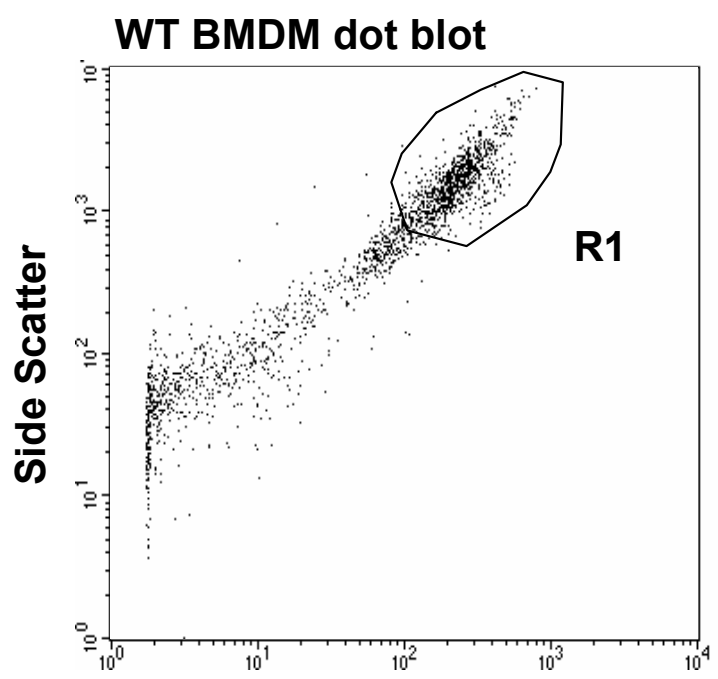

Forward Scatter

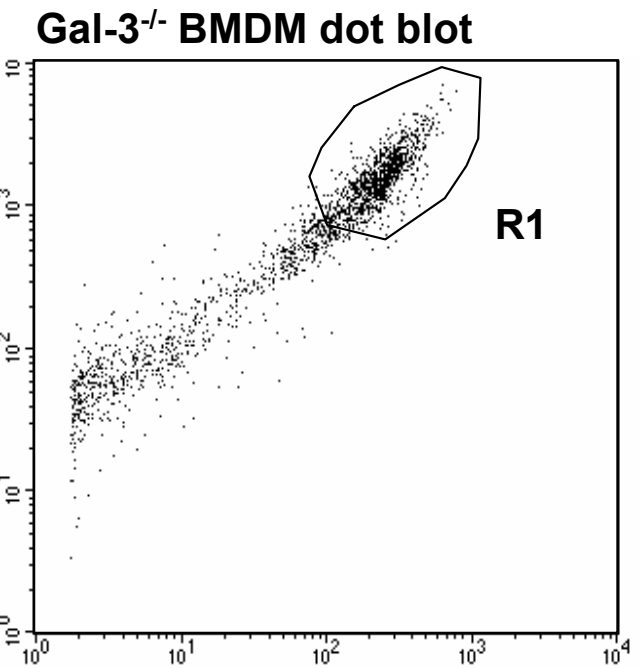

Figure 6.5 (A)

BMDMs seeded in 6-well plates were treated with either $S$. pn or E. coli for up to $1 \mathrm{hr}$, washed with PBS and lifted off the bottom of the well using 5mM EDTA. (A) Forward and side scatter properties of WT and galectin-3-- BMDMs. The population of BMDMs gated on during FACS analysis is indicated by R1. 


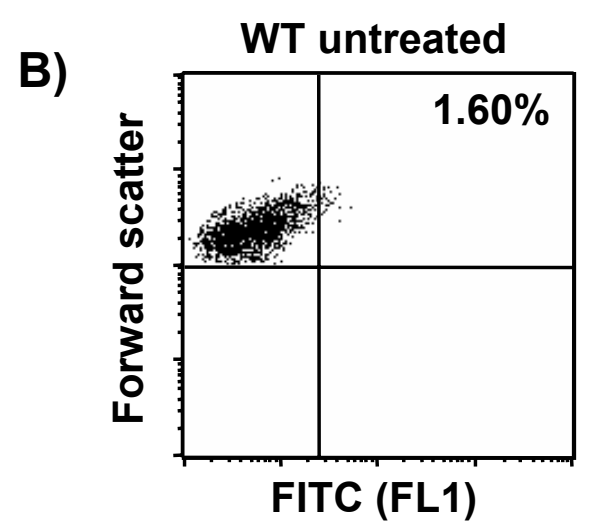

WT + FITC-S. pn (60 min)

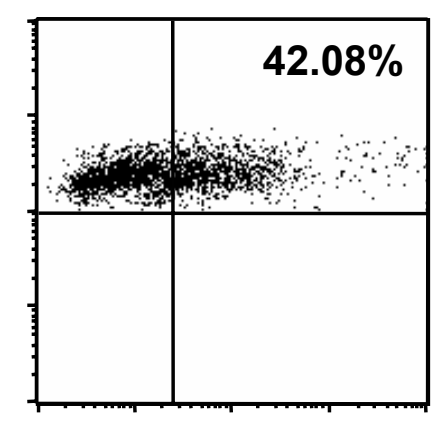

WT + FITC-E. coli (60 min)

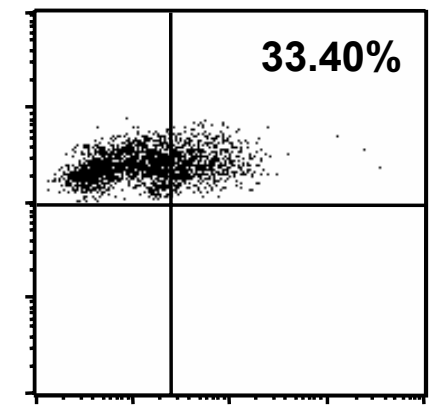

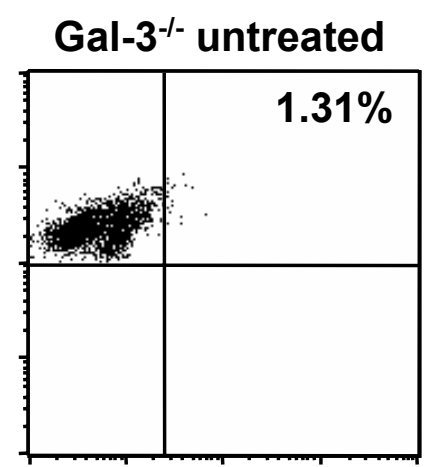

Gal-3-1- + FITC-S. pn (60 min)

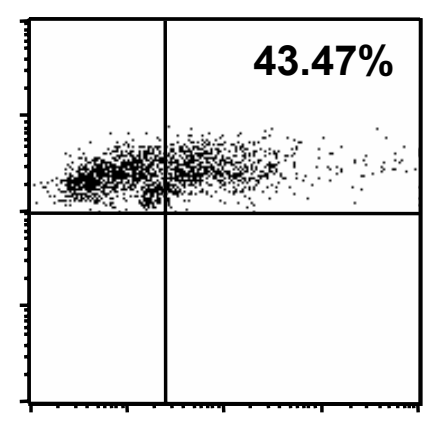

Gal-3-l- + FITC-E. coli (60 min)

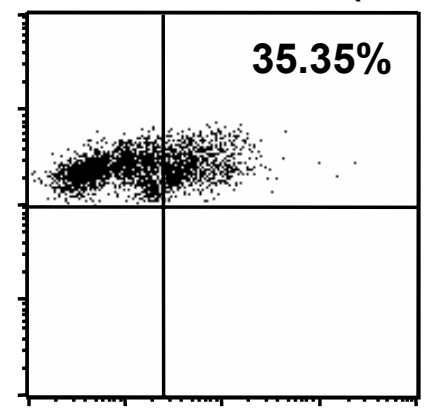

Figure 6.5 (B)

(B) Representative forward scatter versus FL1 dot blots of WT and galectin-3-BMDM treated for 60 min with 10:1 ratio of FITC-S. pn or FITC-E. coli. Gated on macrophages according to forward and side scatter properties (R1). \% in upper right quadrant indicates macrophages that have phagocytosed FITC-labelled bacteria. 
C)

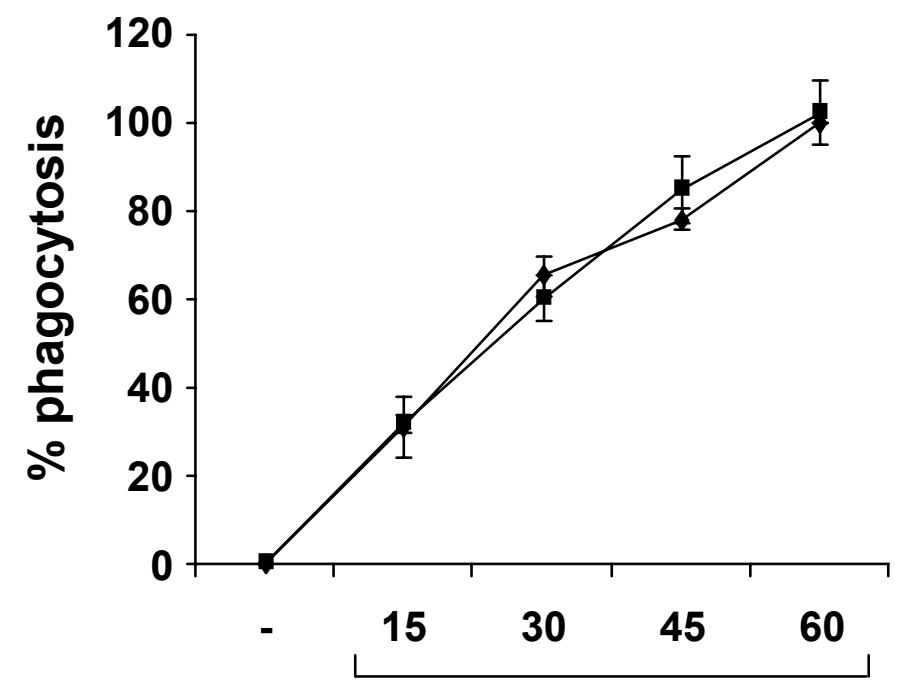

FITC-S. pn (mins)

\section{D)}

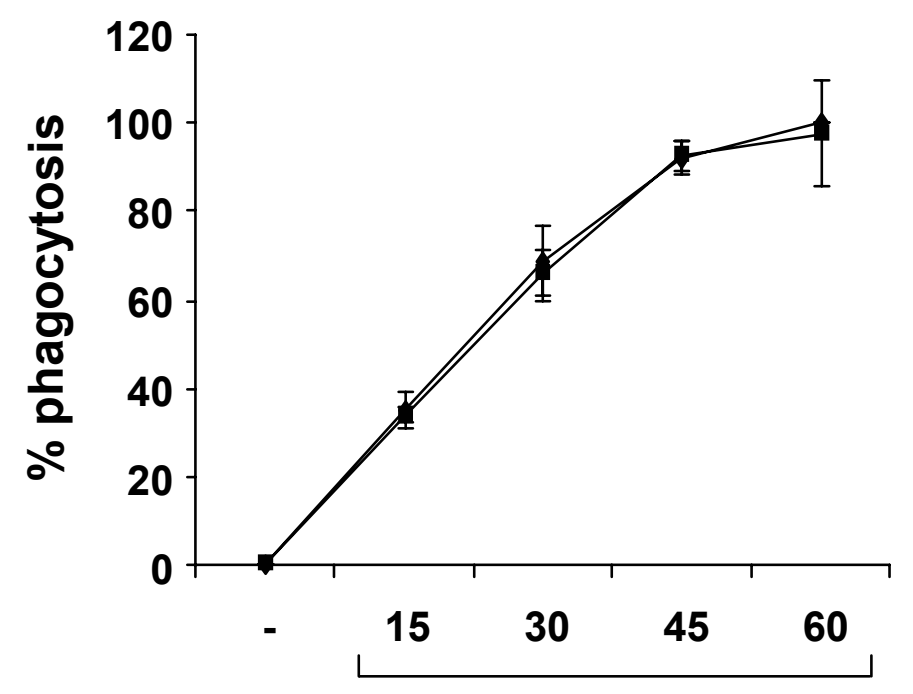

FITC-E. coli (mins)

Figure 6.5 (C, D)

Phagocytosis of a 10:1 ratio of opsonised FITC-S. pn (C) and FITC-E. coli (D) by WT $(\bullet)$ and galectin-3 ${ }^{-/}(\square)$ BMDM measured using FACS analysis. Gated on macrophages according to forward and side scatter properties (R1). Results represent the mean percentage phagocytosis (compared to WT $60 \mathrm{~min})(\mathrm{n}=3)$. 


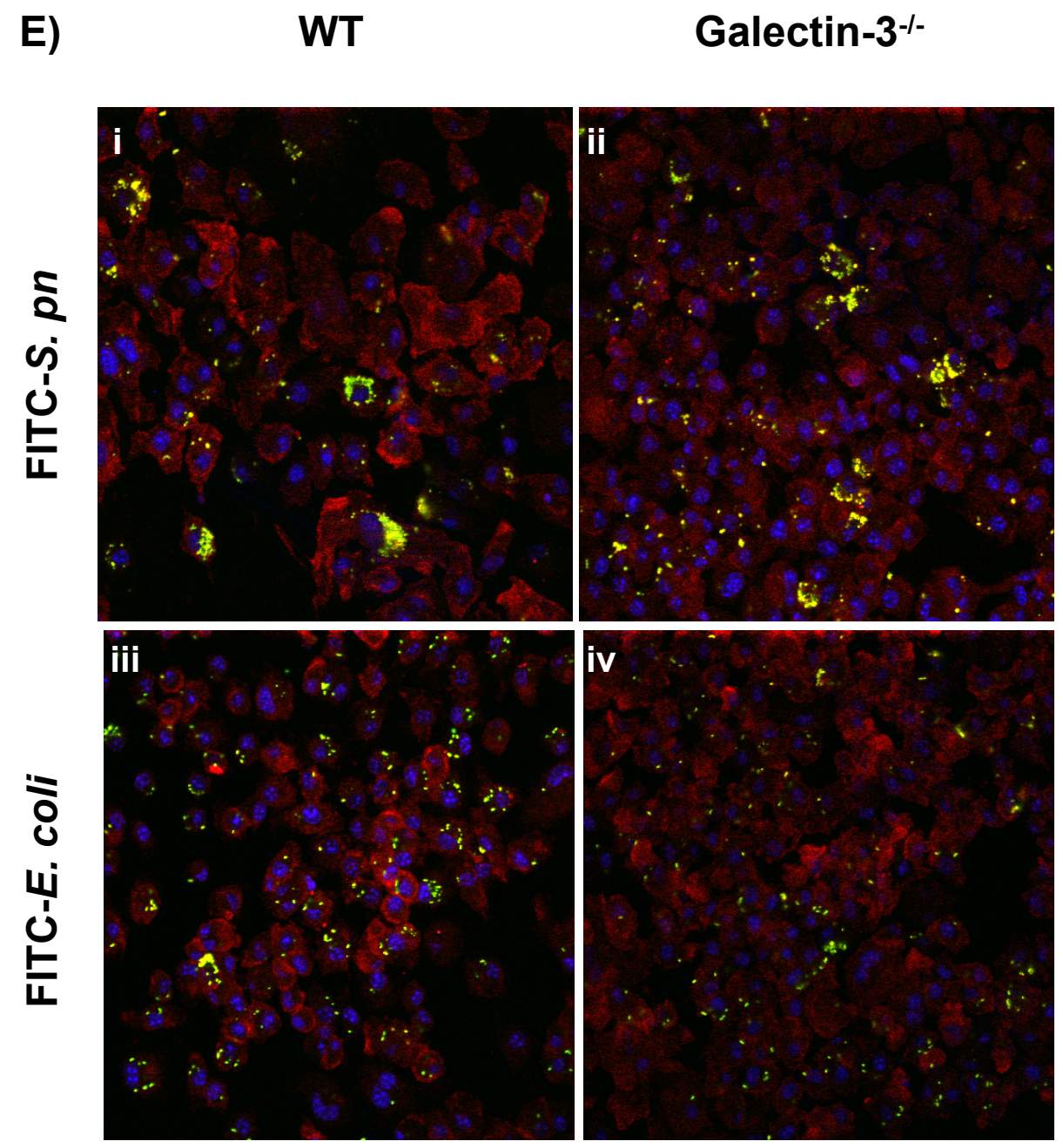

Figure 6.5 (E)

(E) WT and galectin-3-- BMDM incubated with a 10:1 ratio of opsonised FITC$S$. pn (i and ii) or a 20:1 ratio of opsonised FITC-E. coli (iii and iv) and stained with rhodamine phalloidin. Images chosen are representative from 6 independent experiments. 


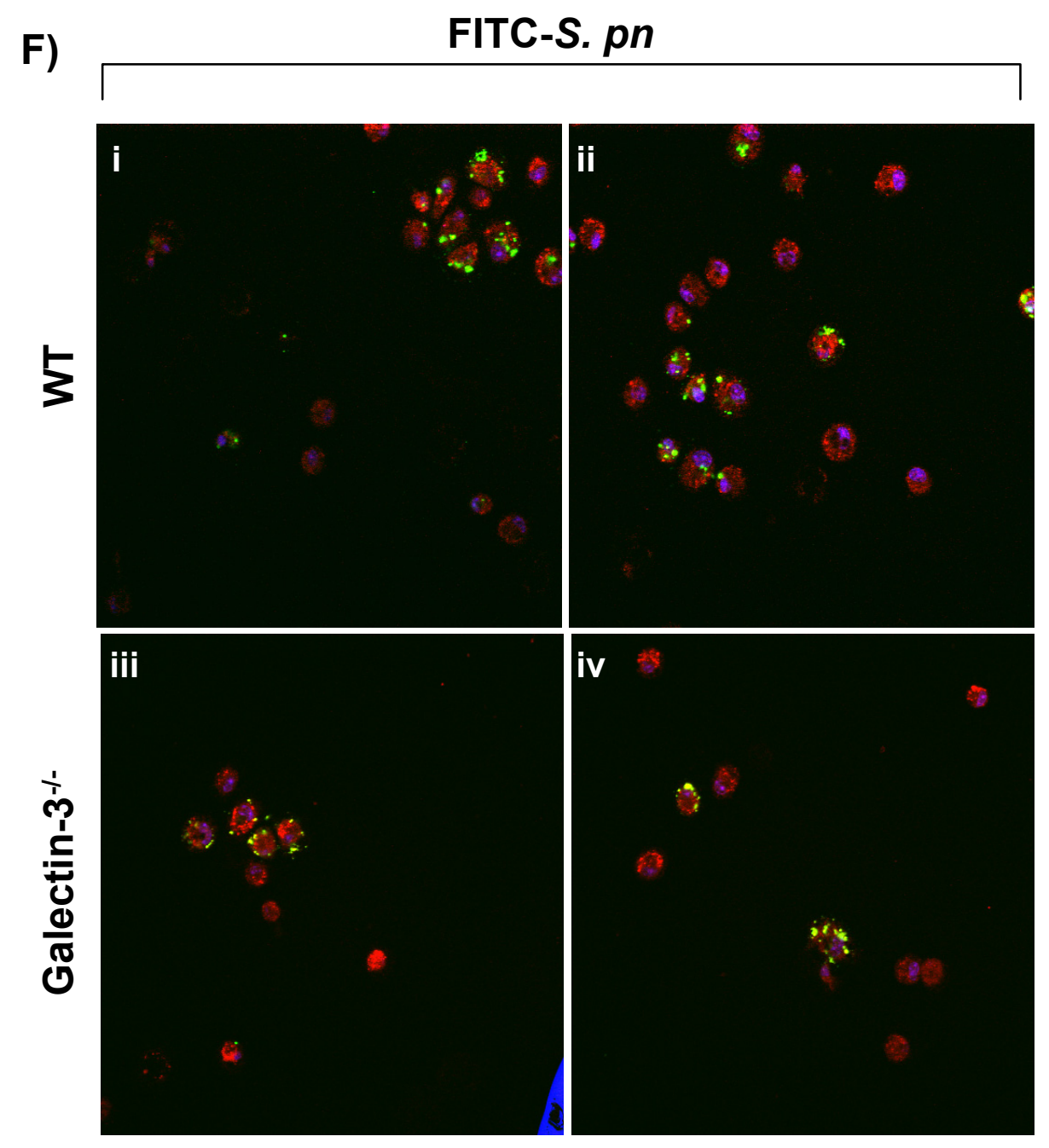

Figure 6.5 (F)

(F) WT (i and ii) and galectin-3-- (iii and iv) alveolar macrophages incubated with a 10:1 ratio of opsonised FITC-S. $p n$ and stained with rhodamine phalloidin. Images chosen are representative from 2 independent experiments. 
Ten random fields were photographed from each slide. Figures $6.5 \mathrm{E}$ and $\mathrm{F}$ show representative images from each slide. Wild type and galectin- $3^{-/-}$BMDMs (figure 6.5E) phagocytosed similar numbers of $S$. pn (i and ii) or E. coli (iii and iv) and wild type and galectin- $3^{-/-}$alveolar macrophages treated with $S . p n$ also exhibit similar levels of phagocytosis (figure 6.5F). Together, these studies indicate no significant phagocytosis defect in galectin- $3^{-/-}$mice of either $S$. pn or E. coli pneumonia.

\subsubsection{Phagocytosis of apoptotic human neutrophils by mouse BMDMs}

Phagocytosis of apoptotic cells is crucial for the resolution of inflammation by protecting tissues from exposure to inflammatory and immunogenic components of dying cells (Savill et al., 2002). Therefore, the increased vasculature damage and bacteraemia in galectin- $3^{-/-}$mice could be due to ineffective clearance of apoptotic cells by galectin- $3^{-/-}$macrophages.

Human neutrophils were isolated from peripheral blood by density gradient centrifugation as described in Materials and Methods. Apoptotic human neutrophils were prepared by culturing neutrophils for 24 hours in Iscove's Modified Eagle's Medium (IMDM) containing 10\% autologous serum. At this time point $>90 \%$ of neutrophils were apoptotic as determined by Annexin V staining (data not shown).

Day 1 apoptotic human neutrophils labelled with CellTracker Green were incubated for up to 1 hour in a 10:1 ratio with CellTracker Orange-labelled BMDMs and phagocytosis assessed by FACS analysis. Phagocytosis of apoptotic neutrophils by macrophages increased from 15 minutes to 1 hour incubation. However, phagocytosis of apoptotic cells by galectin- $3^{-/-}$BMDMs was significantly reduced compared to wild type at the 45 minute time point (figure 6.6). 
Figure 6.6 - Galectin-3 may be required for effective phagocytosis of apoptotic human neutrophils by BMDM.

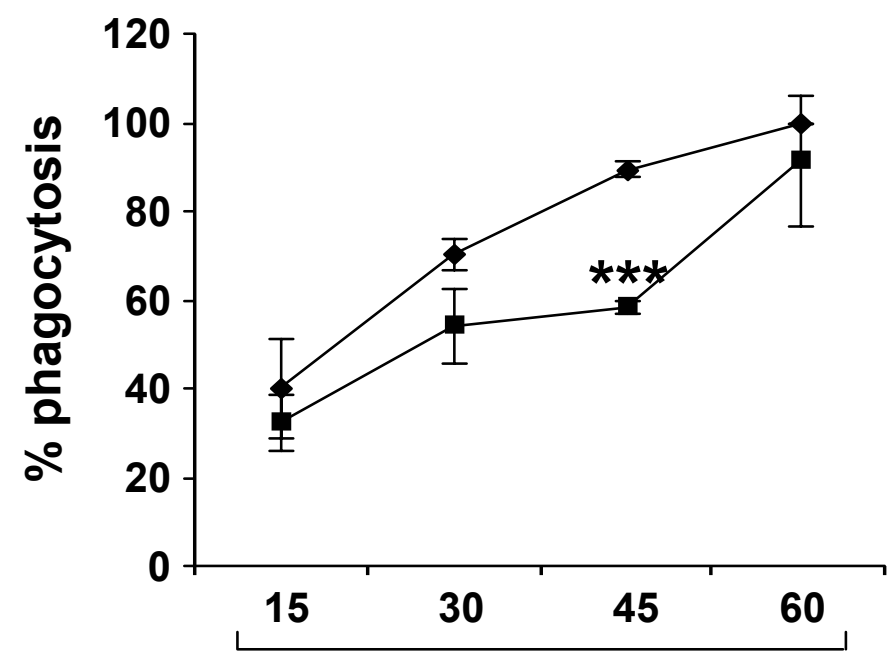

Apoptotic human neutrophil (mins)

\section{Figure 6.6}

Phagocytosis of a 10:1 ratio of apoptotic human neutrophils by WT ( ) and galectin-3-- (- BMDM measured using FACS analysis. Results represent the mean percentage phagocytosis (compared to WT $60 \mathrm{~min})(\mathrm{n}=3)$. ***, $\mathrm{P}<$ 0.0001 compared to WT $45 \mathrm{~min} . \mathrm{P}=0.52$ (ns), 0.09 (ns) and 0.52 (ns) for 15, 30 and 60 min respectively. 


\subsubsection{Exogenously added recombinant galectin-3 causes human neutrophil activation and the generation of ROS}

The professional phagocytic cells recruited at early time points following pneumonia are neutrophils. One hypothesis is that galectin-3 secreted from resident and recruited leukocytes can work in a paracrine manner to activate recruited neutrophils, and augment the clearance of invading bacteria. Upon activation, neutrophils release ROS and upregulate CD11b and shed L-selectin (CD62L). To further elucidate the mechanisms whereby galectin-3 activates neutrophils, human peripheral blood neutrophils were treated with galectin-3 and the above markers of neutrophil activation were assessed.

Human neutrophils were isolated from whole blood as described in Materials and Methods. ROS release was assessed by two methods, cytochrome $\mathrm{C}$ reduction which measures the $\mathrm{O}_{2}^{-}$metabolite (Dahlgren and Karlsson, 1999), and DHR fluorescence which measures the $\mathrm{H}_{2} \mathrm{O}_{2}$ metabolite (Walrand et al., 2003). Details of each protocol can be found in Materials and Methods.

To measure superoxide release, human neutrophils were primed with 0.1 to $10 \mu \mathrm{g} / \mathrm{ml}$ recombinant human galectin-3 or $1 \mu \mathrm{M}$ platelet-activating factor (PAF) as a positive control for 15 minutes. Subsequently, neutrophils were activated with $0.1 \mu \mathrm{M}$ N-Formyl-Methionine-Leucine-Phenylalanine (fMLP) in $1 \mathrm{mg} / \mathrm{ml}$ cytochrome $\mathrm{C}$ for 15 minutes (figure 6.7A). Neutrophils primed with galectin-3 followed by fMLP produced significantly more superoxide compared with neutrophils treated with fMLP alone.

To measure $\mathrm{H}_{2} \mathrm{O}_{2}$ release, human neutrophils were incubated with the fluorescent dye DHR for 5 minutes, primed with $10 \mu \mathrm{g} / \mathrm{ml}$ recombinant mouse or human galectin-3 or $1 \mu \mathrm{M}$ PAF as a positive control for 15 minutes prior to activation with $0.1 \mu \mathrm{M}$ fMLP for 15 minutes. Figure 6.7B illustrates forward and side scatter properties of untreated human peripheral blood neutrophils and neutrophils treated with PAF, mouse or human galectin-3 followed by activation with fMLP. Activated neutrophils undergo a shape change as demonstrated by altered forward and side scatter properties in flow cytometry. Figures $6.7 \mathrm{C}$ and D display representative histograms and pooled data showing increased DHR fluorescence following fMLP in cells primed with galectin-3 compared with fMLP alone. 
Figure 6.7 - Exogenously added recombinant galectin-3 primes fMLP-mediated superoxide release from human peripheral blood neutrophils.

A)

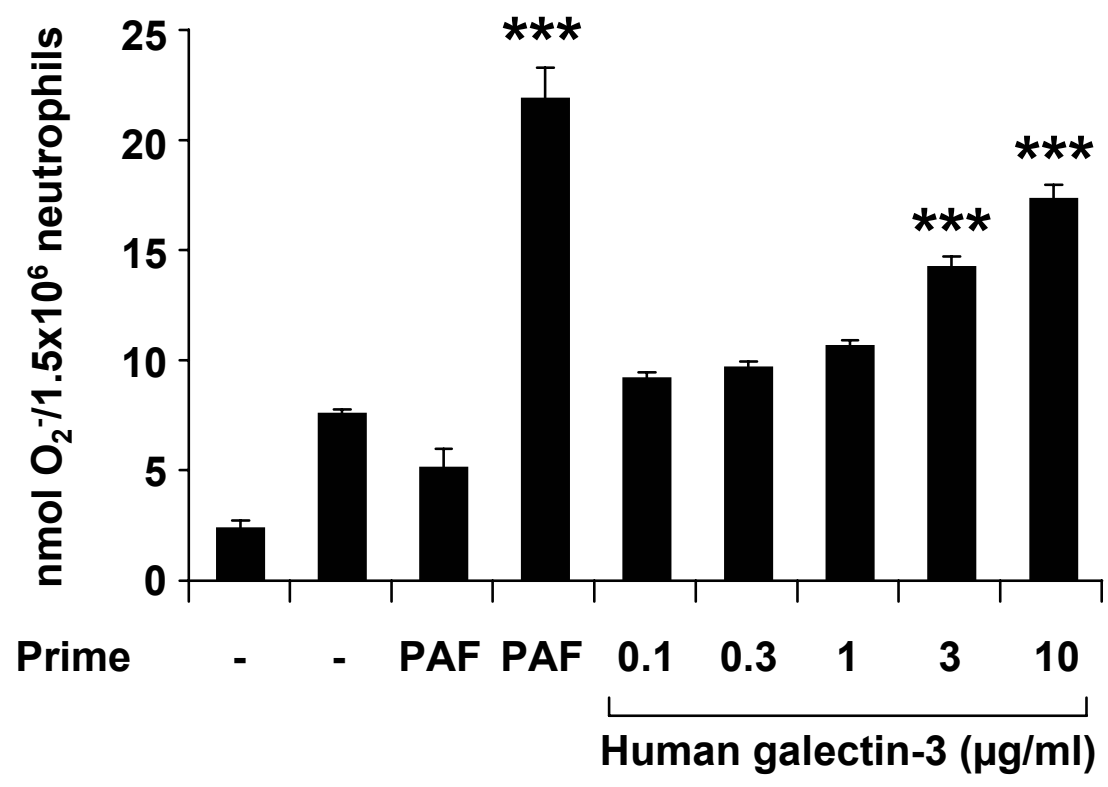

Activate (fMLP)

Figure 6.7 (A)

Cytochrome $\mathrm{C}$ assay. (A) Human peripheral blood neutrophils were incubated for 15 min at $37^{\circ} \mathrm{C}$ with priming agent PAF $(1 \mu \mathrm{M})$ or human recombinant galectin-3 $(1-10 \mu \mathrm{g} / \mathrm{ml})$ followed by a $15 \mathrm{~min}$ incubation with $1 \mathrm{mg} / \mathrm{ml}$ cytochrome C containing the activator $0.1 \mu \mathrm{M}$ fMLP $(+)$ or PBS control (-). Free radical generation was measured by a colour change of cytochrome $\mathrm{C}$ using a scanning spectrophotometer $500 \mathrm{~nm}-600 \mathrm{~nm}$ and concentration of $\mathrm{O}_{2}^{-}$release was calculated $(\mathrm{n}=6){ }^{* * *}, \mathrm{P}<0.0001$ compared to treatment with fMLP alone. 
B)
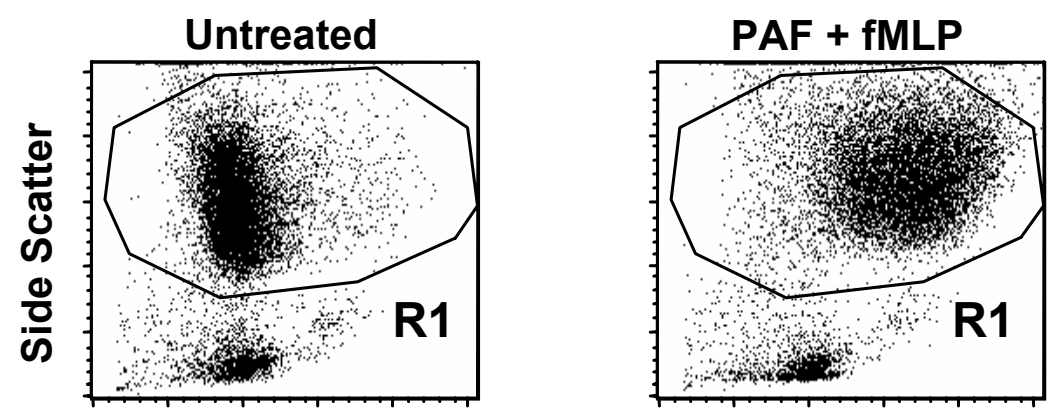

Forward Scatter
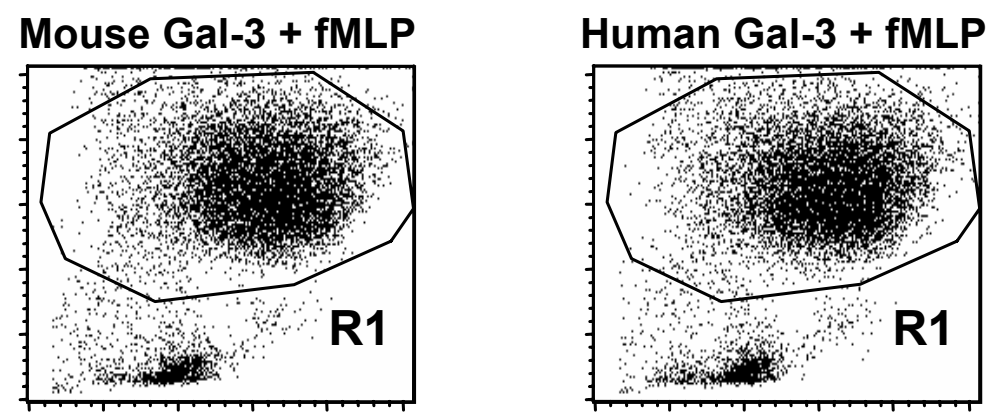

C)

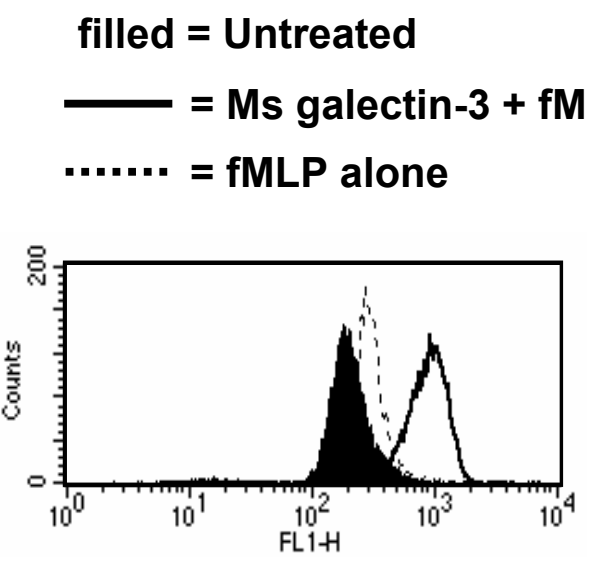

DHR fluorescence filled $=$ Untreated

$-=$ Hu galectin $-3+$ fMLP

....... = fMLP alone

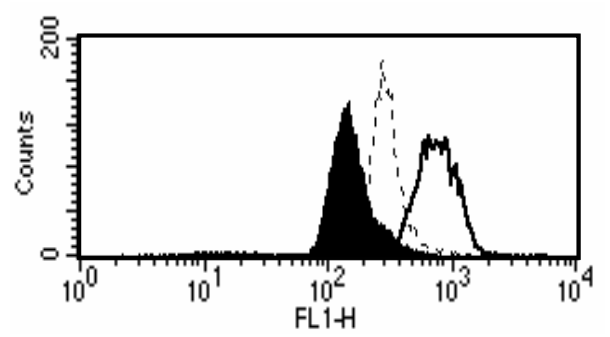

DHR fluorescence

Figure 6.7 (B,C)

Dihydrorhodamine (DHR) fluorescence. Representative dot blots (B) and histograms (C). Human peripheral blood neutrophils incubated at $37^{\circ} \mathrm{C}$ with $1 \mu \mathrm{M}$ DHR for $5 \mathrm{~min}$ followed by a $15 \mathrm{~min}$ incubation with priming agent PAF $(1 \mu \mathrm{M})$ or mouse (Ms) or human $(\mathrm{Hu})$ recombinant galectin-3 $(10 \mu \mathrm{g} / \mathrm{ml})$ then a $15 \mathrm{~min}$ incubation with $0.1 \mu \mathrm{M}$ fMLP or PBS control. Free radical generation was measured by DHR fluorescence using a BD FacsCalibur. 


\section{D)}

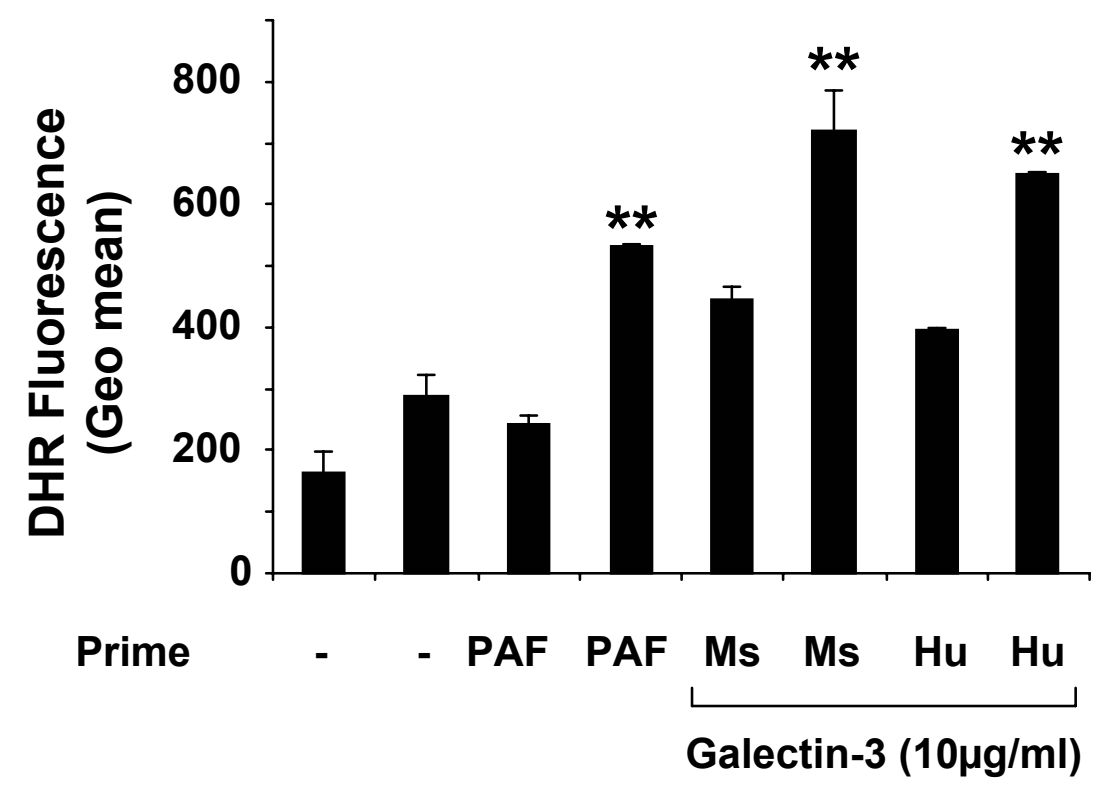

\section{Activate (fMLP)}

Figure 6.7 (D)

DHR fluorescence. (D) Human peripheral blood neutrophils were incubated at $37^{\circ} \mathrm{C}$ with $1 \mu \mathrm{M}$ DHR for $5 \mathrm{~min}$ followed by a $15 \mathrm{~min}$ incubation with priming agent PAF $(1 \mu \mathrm{M})$ or mouse $(\mathrm{Ms})$ or human $(\mathrm{Hu})$ recombinant galectin-3 $(10 \mu \mathrm{g} / \mathrm{ml})$ then a $15 \mathrm{~min}$ incubation with $0.1 \mu \mathrm{M}$ fMLP (+) or PBS control (-). Free radical generation was measured by DHR fluorescence using a BD FacsCalibur $(\mathrm{n}=3)$. **, $\mathrm{P}<0.01$ compared to treatment with fMLP alone. 
When primed with galectin-3 followed by fMLP, the level of free radical generation from isolated human peripheral blood neutrophils exceeded that of neutrophils treated with fMLP alone (figure 6.7A, C and D) suggesting that galectin3 is acting as a neutrophil priming agent.

Subsequent experiments were performed to investigate adhesion molecule expression on human peripheral blood neutrophils in response to exogenous galectin3. Human whole blood was treated with $0.1 \mu \mathrm{M}$ fMLP, $100 \mathrm{ng} / \mathrm{ml}$ LPS or various concentrations of recombinant human galectin-3 followed by incubation with antiCD62L-PE and anti-CD11b-APC antibodies. Red blood cells were lysed in FACS lysing solution, samples were washed and fixed in 1\% paraformaldehyde (PFA). Samples were analysed by FACS analysis after gating on the neutrophil population by forward and side scatter properties (figure 6.8A). Neutrophils activated with galectin-3 undergo a shape change as demonstrated by altered forward and side scatter properties (figure 6.8A). Figures $6.8 \mathrm{~B}, \mathrm{C}$ and $\mathrm{D}$ display representative histograms and pooled data showing decreased L-selectin expression and increased CD11b expression following treatment with galectin-3, fMLP or LPS. Together these experiments prove that not only can galectin-3 prime neutrophils to subsequent activation by fMLP, it can also cause direct activation of neutrophils. 
Figure 6.8 - Exogenously added recombinant galectin-3 mediates L-selectin (CD62L) shedding and CD11b upregulation on human peripheral blood neutrophils.

A)

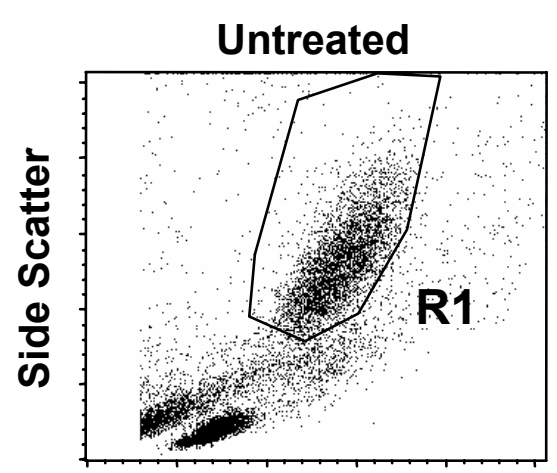

$10 \mu \mathrm{g} / \mathrm{ml}$ Galectin-3

Forward Scatter

B)

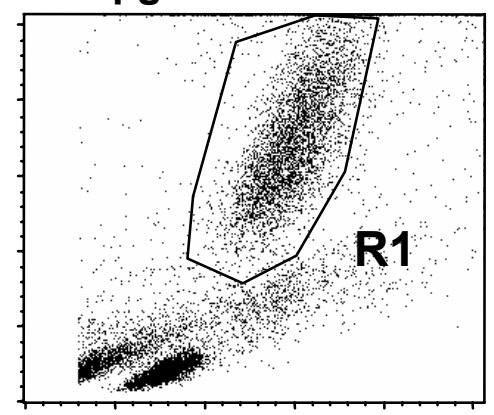
= Human neutrophils + fMLP

i)

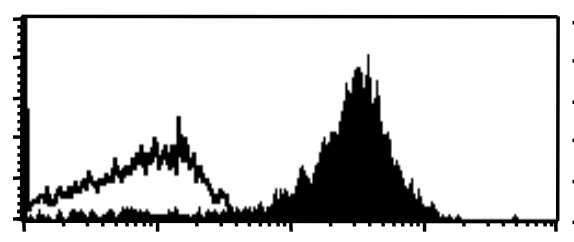

L-selectin (CD62L-PE)

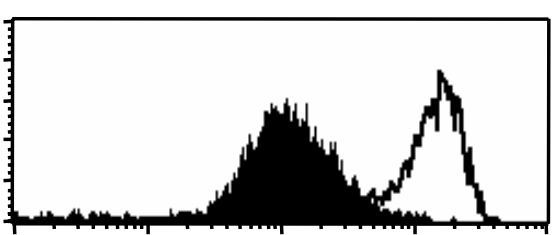

CD11b-APC

$-=$ Human neutrophils $+10 \mu \mathrm{g} / \mathrm{ml}$ gal-3

ii)

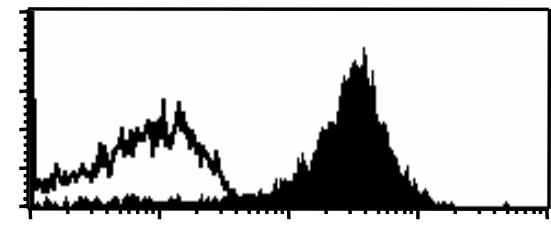

L-selectin (CD62L-PE)

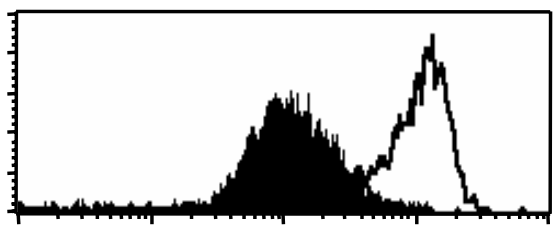

CD11b-APC

Figure 6.8 (A, B)

Adhesion molecule expression. Human whole blood, treated with $0.1 \mu \mathrm{M}$ fMLP, or $10 \mu \mathrm{g} / \mathrm{ml}$ recombinant human galectin-3, was incubated with anti-CD62L-PE and anti-CD11b-APC. (A) Samples gated on human neutrophils (R1) were analysed by FACS analysis. (B) Representative histograms showing L-selectin shedding and $\mathrm{CD} 11 \mathrm{~b}$ upregulation on human neutrophils following treatment with fMLP (i) or galectin-3 (ii). Filled histogram indicates untreated blood. 


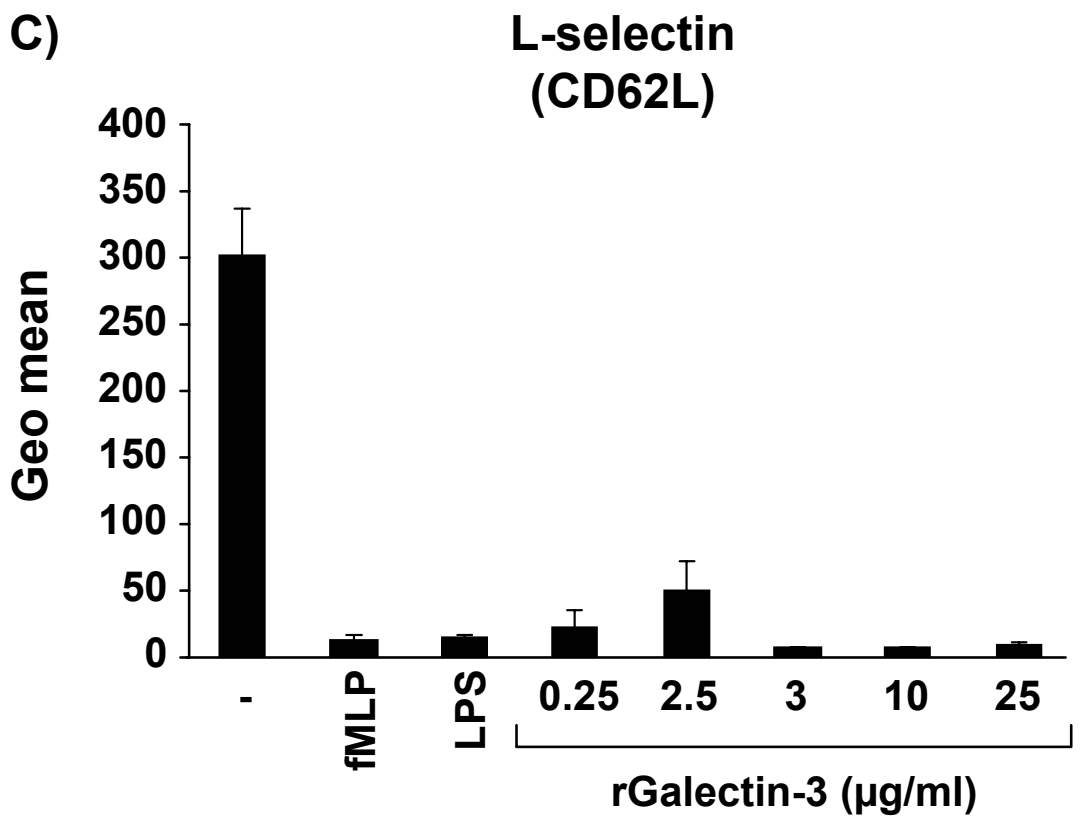

D) CD11b

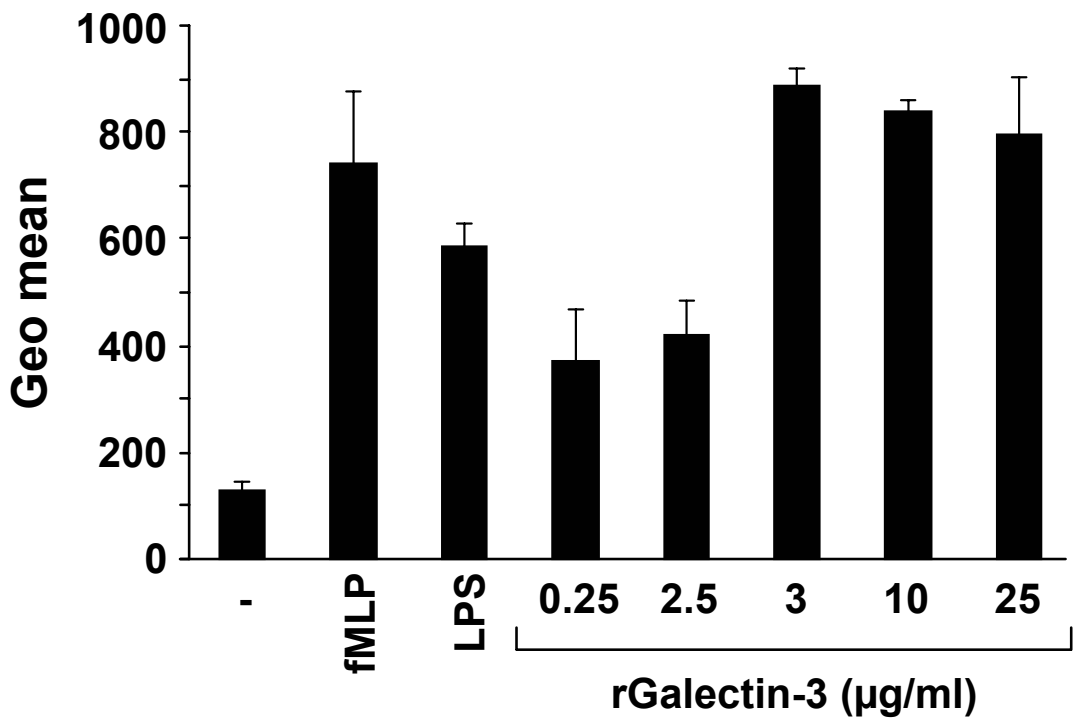

Figure 6.8 (C, D)

Human whole blood, treated with $0.1 \mu \mathrm{M}$ fMLP, $100 \mathrm{ng} / \mathrm{ml}$ LPS or various concentrations of recombinant human galectin-3, was incubated with antiCD62L-PE and anti-CD11b-APC. Samples gated on human neutrophils (R1) were analysed by FACS analysis (C) L-selectin shedding and (D) CD11b upregulation on human neutrophils $(n=6)$. For all treatments $\mathrm{P}<0.01$ compared to untreated. 


\subsubsection{Purification of mouse bone marrow neutrophils}

Subsequent experiments were carried out to address the role of galectin- 3 in the activation and apoptosis of mouse neutrophils from wild type and galectin- $3^{-/-}$ mice. In addition, phagocytosis of apoptotic mouse neutrophils by macrophages and phagocytosis of bacteria by mouse neutrophils was assessed.

The number of neutrophils that can be obtained from mouse blood is very limited, therefore preparation of neutrophils from bone marrow is a useful technique to master. Using this technique, neutrophils from transgenic mice can be easily compared with neutrophils from wild type mice. Previous studies have reported that mouse bone marrow neutrophils show very similar morphology and function to neutrophils isolated from mouse whole blood (Boxio et al., 2004). Furthermore, this technique has been shown to yield greater than 90\% neutrophil purity (Looney et al., 2006). Following an infection, this fully competent neutrophil reservoir in the bone marrow is required to rapidly supplement peripheral neutrophils at times of increased demand (Boxio et al., 2004).

Neutrophils prepared from the bone marrow of wild type and galectin- $3^{-/}$ mice were used to examine the role of galectin-3 on neutrophil function. Neutrophil activation, phagocytosis of bacteria and phagcytosis of apoptotic mouse neutrophils by macrophages was assessed using these bone marrow derived neutrophils. There are several described techniques to isolate granulocyte precursors from whole bone marrow using Percoll density gradient centrifugation (Boxio et al., 2004; Itou et al., 2006; Lowell et al., 1996; Tanaka et al., 2004). However, the method described by Looney et al. was utilised in this study as it gave a good yield and purity (Looney et al., 2006). The full method is described in Materials and Methods. Briefly, bone marrow was flushed out using PBS and treated with a short exposure to a hypotonic solution of $0.2 \% \mathrm{NaCl}$ to lyse the red blood cells quickly followed by $1.2 \% \mathrm{NaCl}$ to restore the isotonicity. The solution was then filtered over a $70 \mu \mathrm{m}$ nylon cell strainer and spun on a $62 \%$ Percoll gradient and neutrophils were isolated from the cloudy pellet at the bottom of the tube. To determine neutrophil purity, cytospins were performed and neutrophil preps were incubated with anti-GR-1-PE (clone RB6-8C5) 1:200 and anti-CD11b-APC (clone M1/70) 1:50 for 30 minutes at $4^{\circ} \mathrm{C}$ in the dark and analysed using a BD FacsCalibur. 
Figure 6.9 - Purification of mouse bone marrow neutrophils.

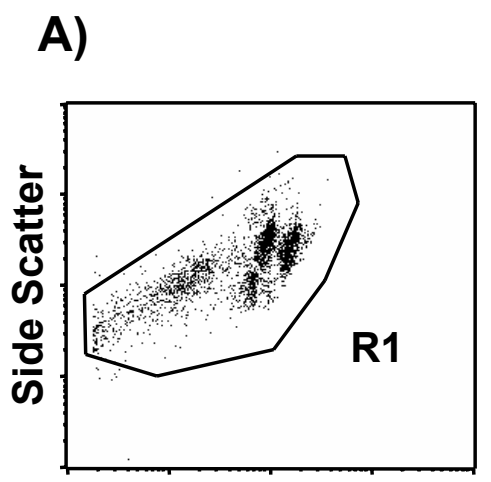

Forward Scatter

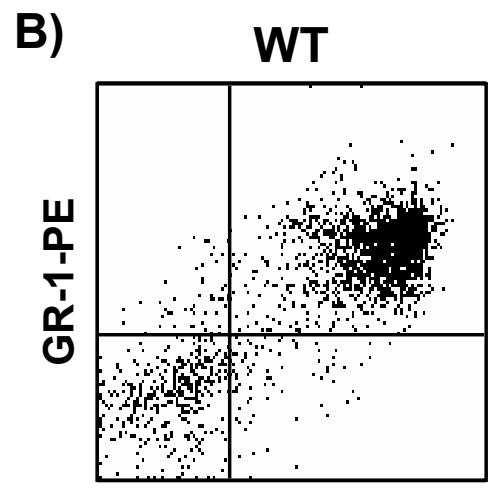

CD11b-APC

C)

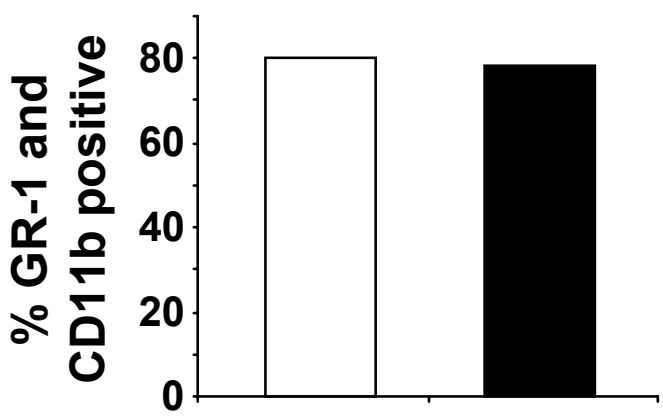

D)

WT

Galectin-3-

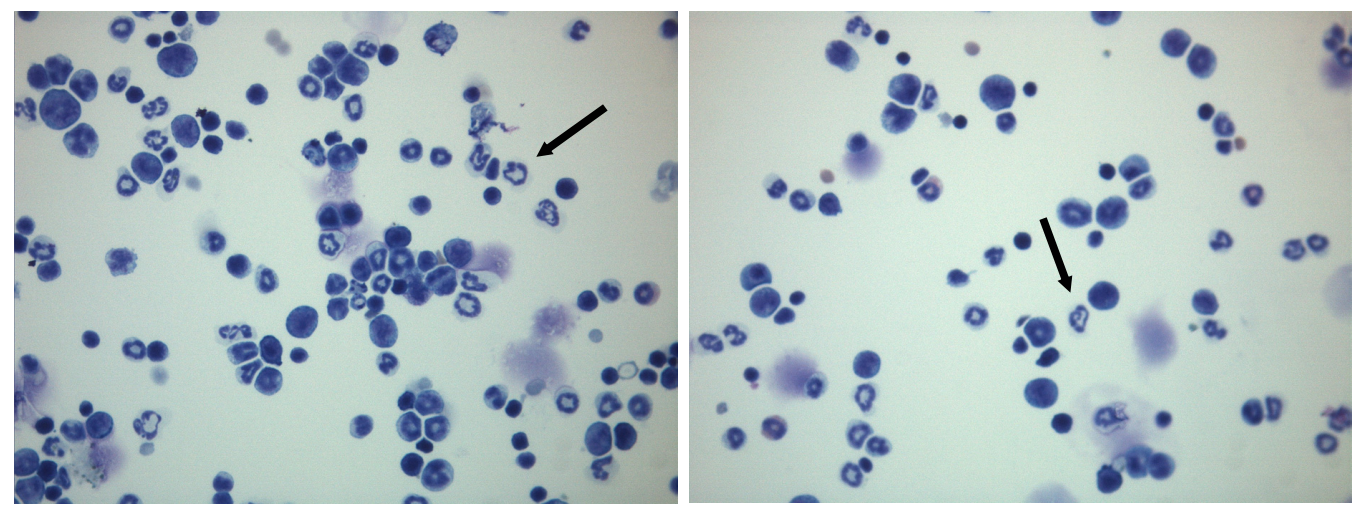

Figure 6.9 (A-D)

Bone marrow neutrophils were prepared as described in Materials and Methods. (A) Representative dot blot showing forward and side scatter properties of a bone marrow neutrophil prep. (B and C) FACS analysis of a WT (white bars) and galectin- $3^{-/}$(black bars) bone marrow neutrophil prep showing GR-1 and CD11b positivity (gated on R1). (D) Representative images of a cytospin from a bone marrow neutrophil prep (Arrows indicate examples of mature neutrophils). 
Figure 6.10 - Apoptosis of WT and galectin-3-- mouse bone marrow derived neutrophils over 96 hours in culture.

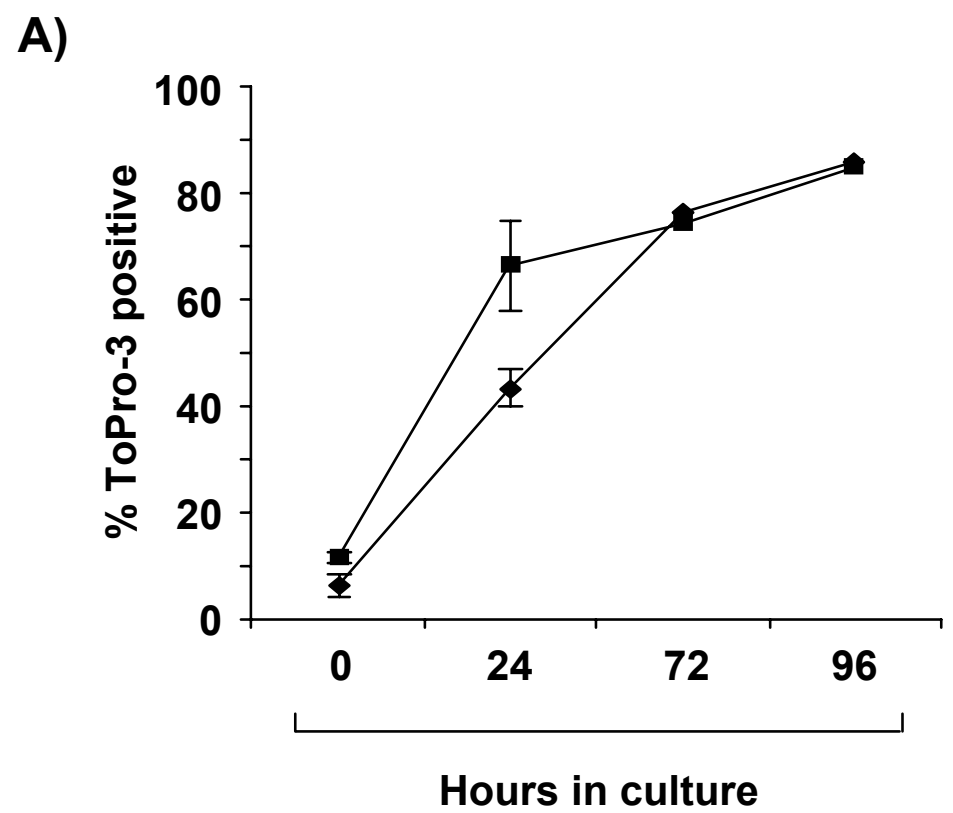

B)

Wild type

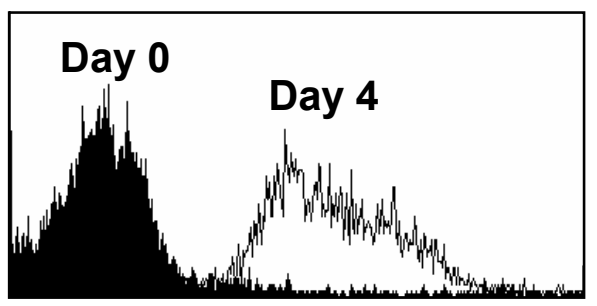

ToPro-3 (FL4)
Galectin-3-1-

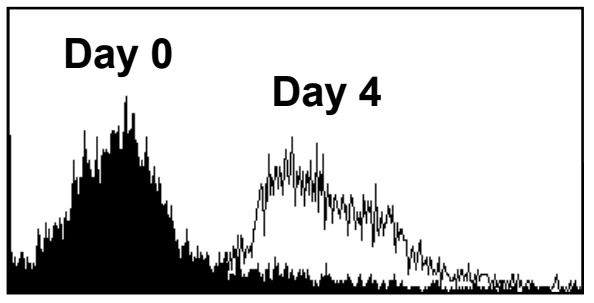

ToPro-3 (FL4)

\section{Figure 6.10 (A, B)}

Bone marrow neutrophils were prepared as described in Materials and Methods and cultured in RPMI containing 10\% FSC for up to four days. At each time point, an aliquot of cells were analysed by flow cytometry and the percentage of dead, ToPro-3 positive, cells was determined. (A) FACS analysis of WT ( ) and galectin-3 ${ }^{-/}(\square)$ neutrophils 24 to 96 hours in culture $(\mathrm{n}=2)$. (B) Histogram overlays demonstrating ToPro-3 positivity of both WT and galectin- $3^{-/-}$ neutrophils after 4 days in culture. 
Figure 6.9A illustrates forwards and side scatter properties of a bone marrow neutrophil prep. The various cell populations observed most likely demonstrate neutrophils that are matured to different extents. However when all cell populations are taken into account (gate R1), the percentage of both GR-1 and CD11b positive cells is approximately $80 \%$ (figure 6.9B and C). Cytospins of these cells displayed neutrophil-type morphology with multi-lobular nuclei (arrows) (figure 6.9D).

\subsubsection{Apoptosis of mouse bone marrow derived neutrophils}

It has been previously reported that human neutrophils become apoptotic after 24 hours in culture (Savill et al., 1989; Dransfield et al., 1994). To assess mouse neutrophil apoptosis in vitro, wild type and galectin- $3^{-/-}$mouse bone marrow derived neutrophils were incubated in RPMI containing 10\% foetal bovine serum over 96 hours. Percentage cell death at various time points was determined by incubating the cells with ToPro-3 and analysing samples by FACS analysis. ToPro-3 positive cells indicate dead cells. At 24 hours, galectin- $3^{-/-}$neutrophils showed greater ToPro-3 positivity compared to wild type neutrophils (figure 6.10A). By 72 hours in culture, around $70 \%$ of both wild type and galectin- $3^{-/-}$neutrophils were dead. Both wild type and galectin- $3^{-/-}$neutrophils are $\sim 90 \%$ ToPro-3 positive after 96 hours in culture (figure 6.10A and B). These results indicated that, in vitro, mouse neutrophils require longer in culture than human neutrophils to become apoptotic and there may be a difference in the rate at which wild type and galectin-3-

/- neutrophils become apoptotic when cultured in vitro, however this needs to be investigated further.

\subsubsection{Phagocytosis of apoptotic mouse neutrophils by mouse BMDMs}

The results in section 6.2.7 indicated that galectin- $3^{-/-}$macrophages may show a defect in phagocytosis of apoptotic neutrophils. However, this was done using apoptotic human neutrophils. Subsequent experiments were performed to assess phagocytosis of day 2 apoptotic wild type mouse neutrophils by BMDM by confocal microscopy. 
Figure 6.11 - Phagocytosis of apoptotic mouse neutrophils by BMDM.

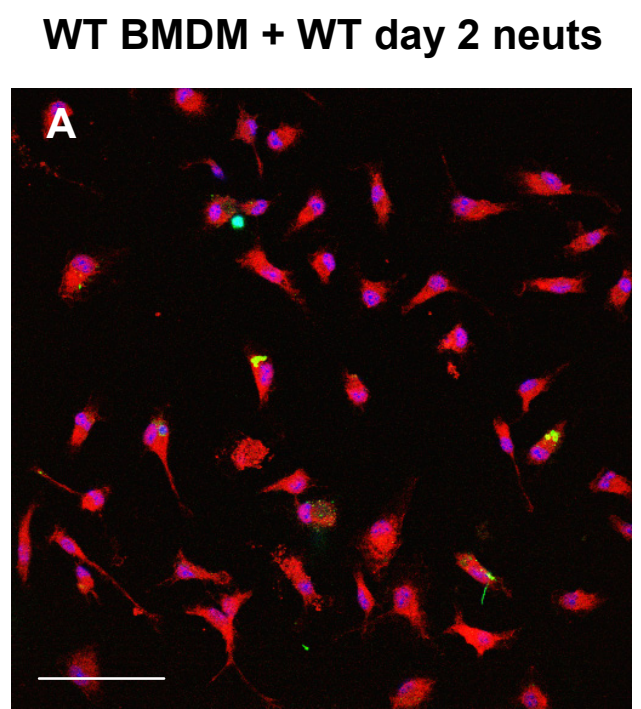

KO BMDM + WT day 2 neuts
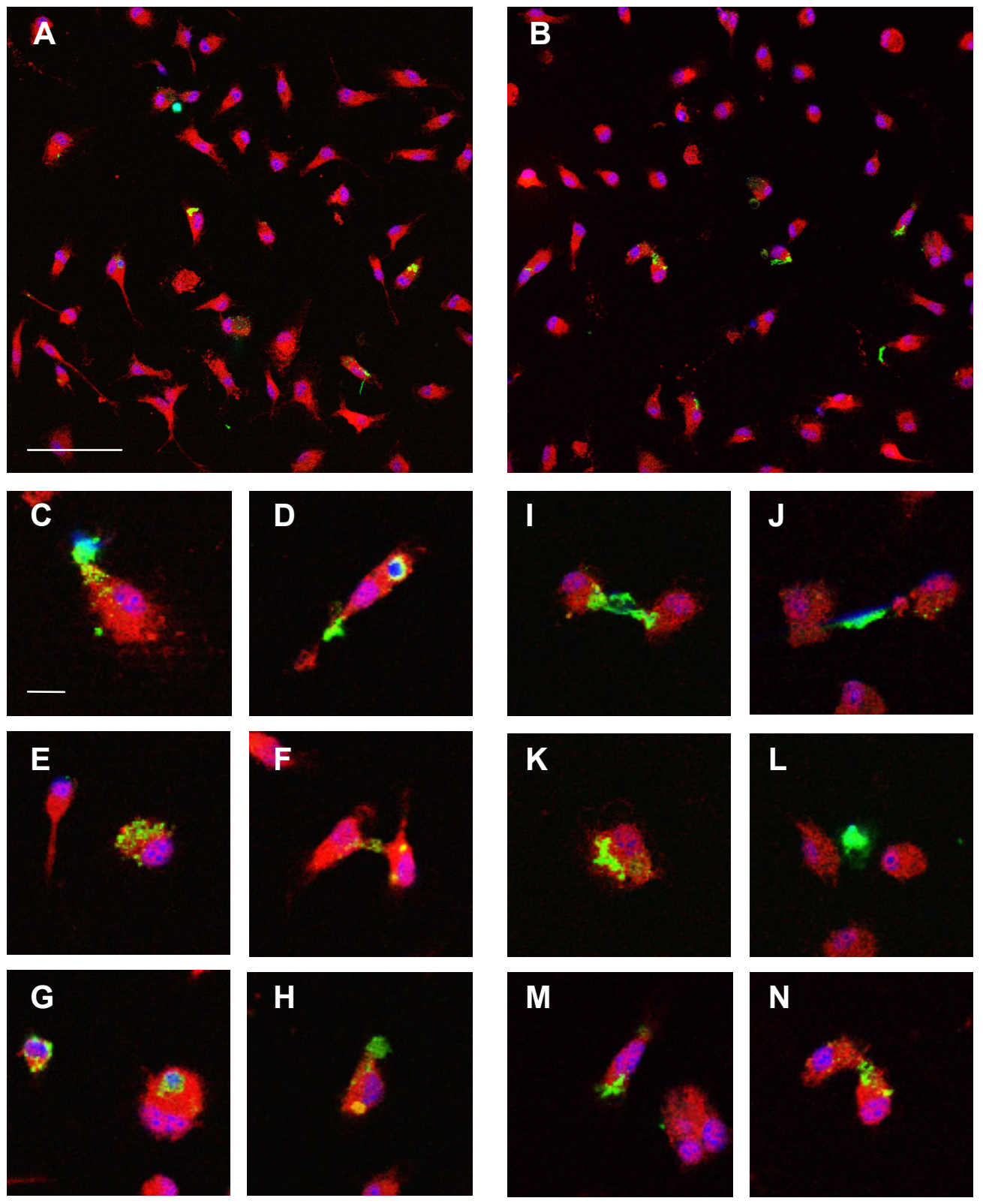

Figure 6.11 (A-N)

Images showing phagocytosis of a 5:1 ratio of day 2 WT mouse bone marrow derived neutrophils by WT (A) and galectin- $3^{-/-}$(B) BMDM after $1 \mathrm{hr}$ incubation. (Scale bar: $50 \mu \mathrm{m})$. (C-N) Selected images of individual WT (C-H) and galectin$3^{--}$(I-N) BMDMs phagocytosing apoptotic WT mouse neutrophils at higher magnification. (Scale bar: $5 \mu \mathrm{m}$ ). 
CellTracker Green-labelled apoptotic neutrophils were incubated for 1 hour in a 5:1 ratio with CellTracker Orange-labelled BMDMs on coverslips. Images were captured using a Leica SP5 confocal microscope and subsequently processed using Adobe Photoshop (figure 6.11). Galectin- $3^{-/-}$BMDMs demonstrated equivalent levels of phagocytosis to wild type BMDMs. Together with the apoptotic human neutrophil data, these results are as yet inconclusive, and more studies need to be carried out before a definitive conclusion can be drawn regarding the effect of galectin-3 on phagocytosis of apoptotic neutrophils.

\subsubsection{Phagocytosis of bacteria by mouse bone marrow neutrophils}

Untreated wild type and galectin- $3^{-/-}$bone marrow neutrophils incubated with opsonised FITC-labelled S. pn or E. coli showed similar levels of phagocytosis indicating that the presence of galectin-3 is not essential for effective phagocytosis in neutrophils. However, after 1 hour incubation with $10 \mu \mathrm{g} / \mathrm{ml}$ recombinant mouse galectin-3 purified using the baculovirus expression system, both wild type and galectin- $3^{-/-}$bone marrow neutrophils enhanced their phagocytic capability (figure 6.12A and B). This indicated that exogenous galectin-3 can stimulate phagocytosis in neutrophils.

This data agrees with the hypothesis that galectin-3 secreted from resident and recruited leukocytes can work in a paracrine manner to activate recruited neutrophils, and augment the clearance of invading bacteria.

\subsubsection{Exogenously added recombinant galectin-3 activates both wild type and galectin- $3^{-1 /}$ mouse peripheral neutrophils}

To compare the activation of wild type versus galectin $-3^{-/-}$neutrophils, mouse whole blood, treated with recombinant mouse galectin-3, was incubated with antiCD11b-APC or anti-GR-1-PE antibodies. Samples were gated on GR-1 positive neutrophils by FACS analysis (figure 6.13A) and high CD11b expression (CD11 $b^{\mathrm{HI}}$ ) on neutrophils in galectin-3-treated mouse whole blood was compared to those of untreated controls (figure 6.13B and C). Both wild type and galectin- $3^{-/-}$neutrophils demonstrated over $90 \%$ CD11b positivity when treated with galectin-3. 
Figure 6.12 - Galectin-3 is not required for effective phagocytosis of $S$. pn or $E$. coli by mouse neutrophils but enhances phagocytosis when added exogenously.
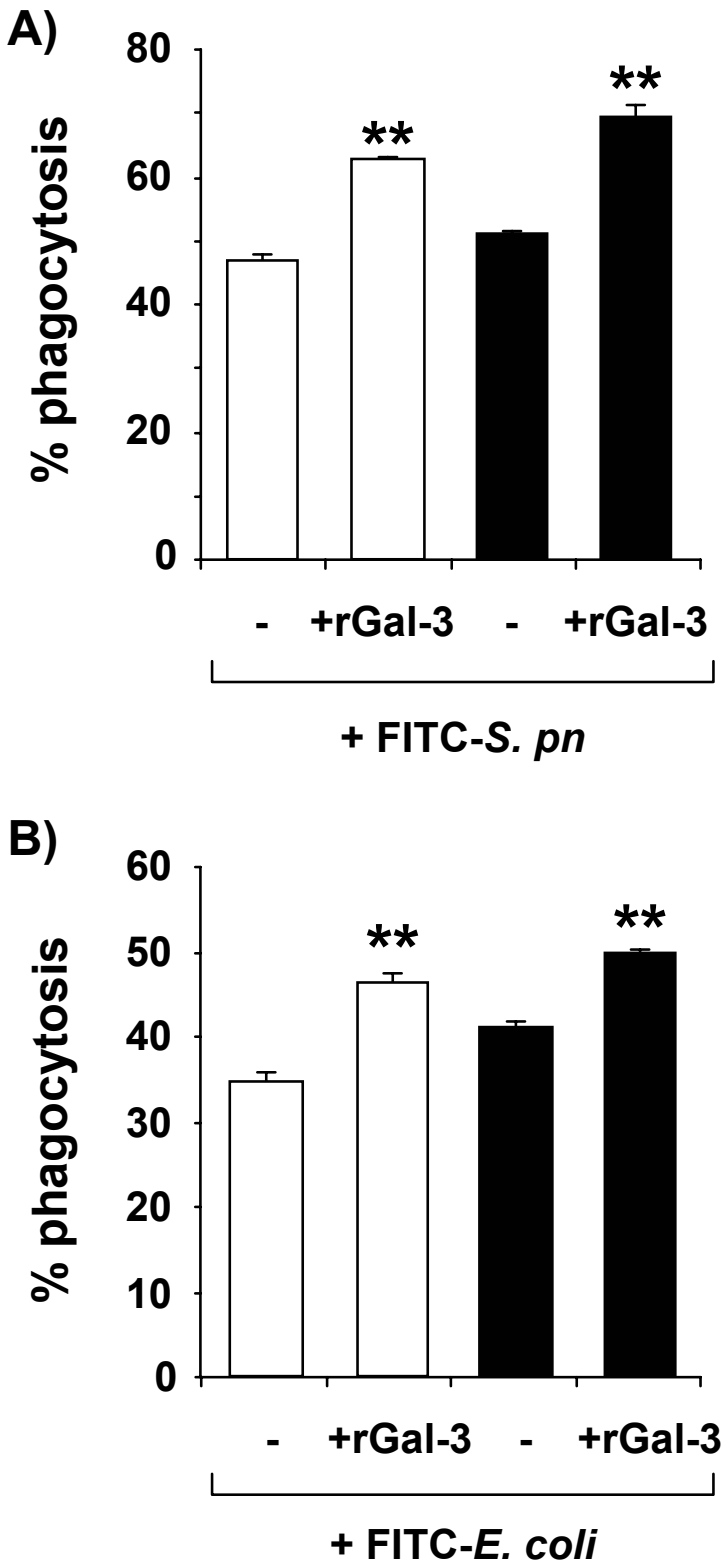

Figure 6.12 (A, B)

WT (white bars) and galectin-3 $3^{-/-}$(black bars) bone marrow neutrophils were prepared as described in Materials and Methods. Half were incubated with $30 \mu \mathrm{g} / \mathrm{ml}$ recombinant mouse galectin- 3 for $1 \mathrm{~h}$ at $37^{\circ} \mathrm{C}$ then incubated with a $10: 1$ ratio of opsonised FITC-S. pn (A) or FITC-E. coli (B) for $1 \mathrm{~h}$ at $37^{\circ} \mathrm{C}$. Samples were analysed using a BD FacsCalibur $(\mathrm{n}=3)$. ${ }^{* *}, \mathrm{P}<0.01$ compared to untreated. 
Figure 6.13 - Exogenously added recombinant galectin-3 increases CD11b expression on both wild type and galectin$3^{-/-}$mouse whole blood neutrophils.

A)

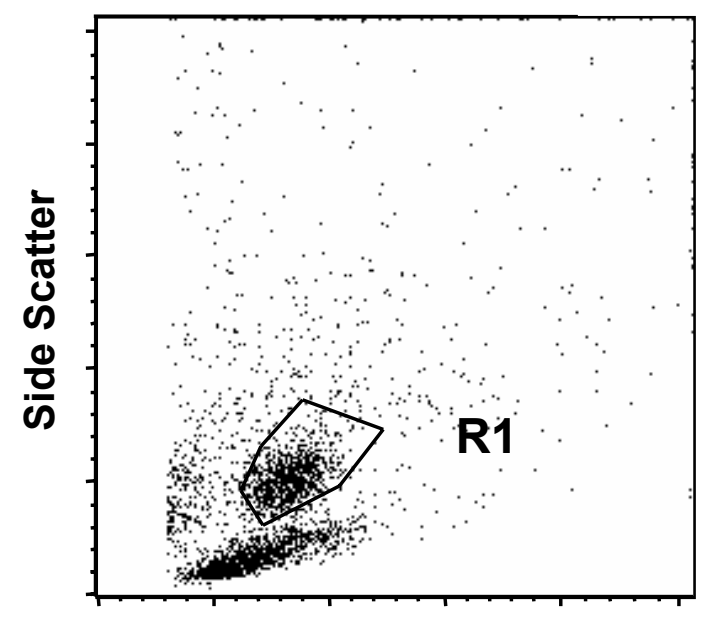

Forward Scatter
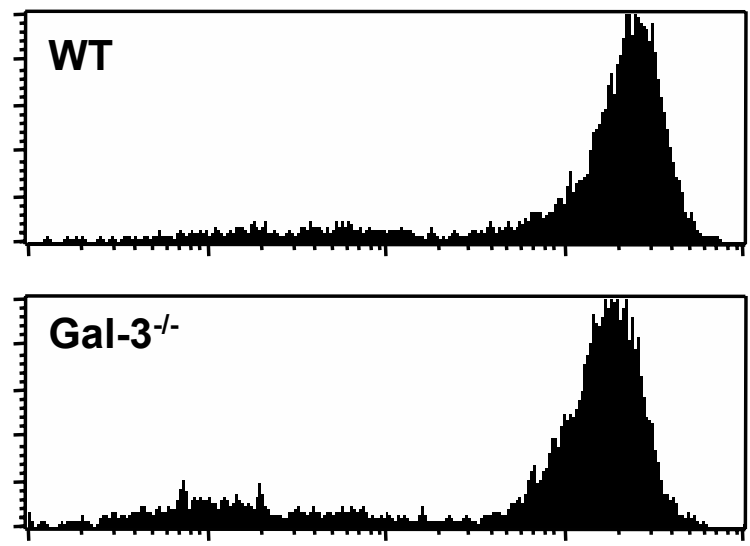

GR-1-PE (FL2)

Figure 6.13 (A)

Mouse whole blood was incubated with anti-GR-1-PE. Samples gated on GR-1 positive neutrophils were analysed by FACS analysis. (A) GR-1 positive neutrophils in mouse whole blood (R1). 

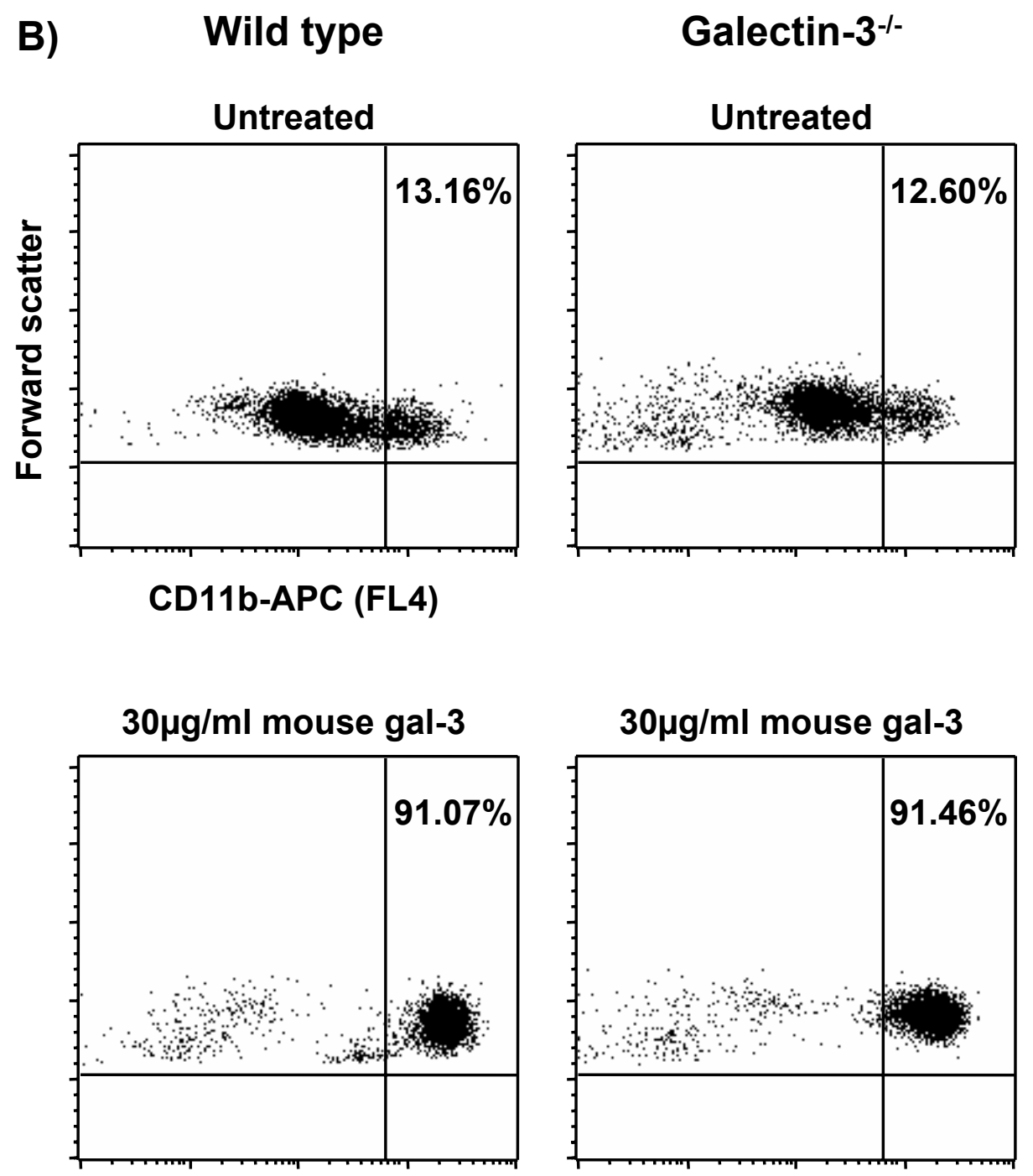

\section{Figure 6.13 (B)}

Mouse whole blood was treated with $30 \mu \mathrm{g} / \mathrm{ml}$ mouse galectin-3 and incubated with anti-CD11b-APC and anti-GR-1-PE. Samples gated on GR-1 positive neutrophils were analysed by FACS analysis. (B) CD11b positivity on neutrophils (gated on R1) from WT and galectin- $3^{-/-}$mouse whole blood, treated with $30 \mu \mathrm{g} / \mathrm{ml}$ mouse galectin-3. \% in upper right quadrant indicates percentage highly expressing CD11b neutrophils. 
C)

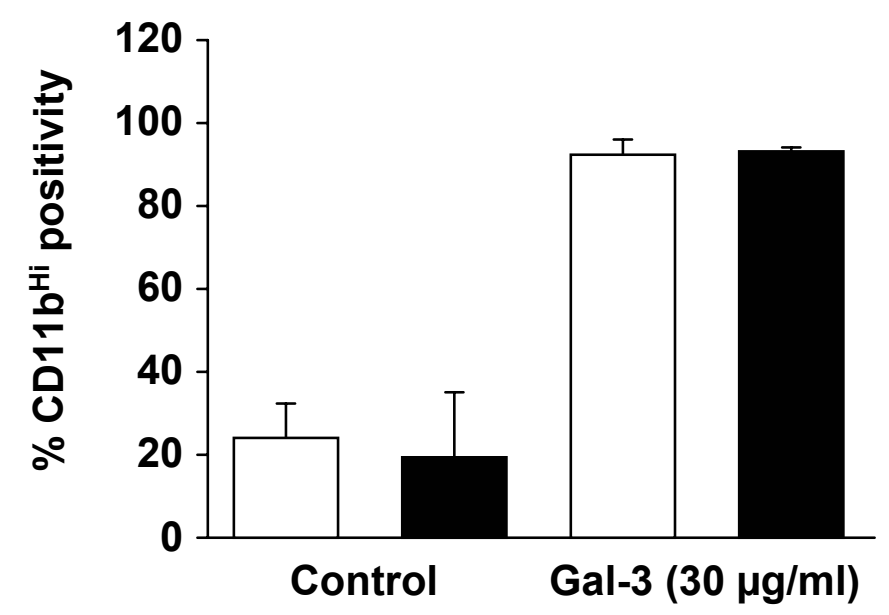

Figure 6.13 (C)

(C) Neutrophils expressing high levels of CD11b $\left(\mathrm{CD} 11 \mathrm{~b}^{\mathrm{HI}}\right)$ from WT (white bars) and galectin- $3^{-/-}$(black bars) mouse whole blood. Whole blood was treated with $30 \mu \mathrm{g} / \mathrm{ml}$ mouse galectin-3 and incubated with anti-CD11b-APC and antiGR-1-PE. Samples gated on GR-1 positive neutrophils were analysed by FACS analysis $(\mathrm{n}=3)$. 
Together with the results using human neutrophils, these data demonstrate that exogenously added galectin-3 can activate both human and mouse neutrophils. Furthermore, exogenous galectin-3 increases CD11b expression on both wild type an galectin- $3^{-/-}$neutrophils to similar extents indicating that regardless of the initial mouse phenotype, neutrophils can be equally activated when exposed to exogenous galectin-3.

\subsection{Discussion}

In vivo, galectin-3 plays an important role in the clearance of pneumococcal pneumonia but not in E. coli pneumonia. Galectin- $3^{-/-}$mice demonstrated a clearance defect of $S$. pn with increased septicaemia and a greater extent of lung damage compared to wild type mice. This phenotype was markedly reduced in an E. coli pneumonia infection. Galectin- $3^{-/-}$macrophages and neutrophils phagocytosed $S . p n$ just as efficiently as wild type. However, addition of exogenous galectin-3 activated neutrophils and increased their ability to phagocytose bacteria. Incubation of wild type macrophages with both $S$. pn and E. coli enhanced NO production which was not seen with galectin- $3^{-/-}$macrophages, and galectin-3 had antimicrobial properties against $S$. pn. This data is consistent with the hypothesis that galectin-3 released from macrophages in response to pneumococcal pneumonia infection can activate neutrophils and enhance their phagocytic capability so aiding the bacterial clearance observed in wild type mice following pneumococcal infection. In addition, galectin3 may aid bacterial clearance by having a direct bactericidal effect on $S$. $p n$.

Data from the in vivo arm of this study demonstrated an increase in bacterial counts in lung homogenate, indicating a bacterial clearance defect, and the presence of bacteria in the blood (an indication of sepsis) of galectin- $3^{-/-}$mice given an acute infection of $S$. $p n$ when compared to strain, age and sex matched wild type mice. Lavages from these galectin- $3^{-/-}$mice contained higher protein concentration, a well established assay of vasculature damage resulting in protein leakage into the lung space. This study also demonstrated reduced neutrophil recruitment in the galectin$3^{-/-}$mouse in response to a pneumococcal infection. Therefore, galectin-3 may act as an adhesion molecule during neutrophil extravasation in response to pneumococcal pneumonia (Sato et al., 2002). Sato et al. also reported that neutrophil extravasation 
following E. coli pneumonia does not involve galectin-3. However, it was not reported whether galectin-3 positive mice developed less severe injury in response to E. coli. In this study, wild type and galectin $-3^{-/-}$mice developed comparable blood sepsis and demonstrated similar levels of bacterial load in response to E. coli. These results show that galectin-3 is important in regulating the host response to $S$. pn while the response to E. coli infection was less pronounced.

Alveolar macrophages and recruited macrophages and neutrophils play a key role in the clearance and killing of invading pathogens. Subsequent in vitro assays were used to test the hypothesis that there may be a phagocytosis, killing or activation defect of galectin- $3^{-/-}$macrophages and/or neutrophils in response to $S . p n$. In vitro phagocytosis assays revealed no phagocytosis defect of $S$. pn or E. coli by either alveolar macrophages or BMDM as tested by both FACS analysis and microscopy. Disruption of the galectin-3 gene in macrophages resulted in a deficit of NO production after incubation with $S$. $p n$ and E. coli. Upon activation, neutrophils release superoxide (free radicals), upregulate CD11b and shed L-selectin (CD62L). Exogenously added galectin-3 increased CD11b and decreased L-selectin expression on human neutrophils from whole blood and increased CD11b expression on both wild type and galectin- $3^{-/-}$neutrophils from whole blood. Furthermore, addition of galectin-3 increased free radical production from human peripheral blood neutrophils. These in vitro data suggest that the presence of galectin-3 is not necessary for successful phagocytosis of bacteria but may be essential for the killing mechanisms used by macrophages and neutrophils, which contribute to the eradication of a pneumonia infection.

Following phagocytosis, the internalised particle is transported to a phagosome containing proteins required for killing internalised pathogens. This requires the maturation of the phagosome to a hydrolytic and acidic phagolysosome, which directly limits pathogen replication. Following phagocytosis of latex beads or Mycobacterium tuberculosis by macrophages galectin-3 accumulates on the cytoplasmic surface of phagocytic cups and phagosomes (Beatty et al., 2002; Garin et al., 2001), where it may have a putative role in regulating phagosome maturation. As the mycobacteria is phagocytosed, galectin-3 associated with the extracellular surface is incorporated at the luminal face of the bacterial phagosome. However, 
over time galectin-3 is increasingly accumulated into the phagosome which would suggest that the additional source of galectin-3 may be vesicle-derived and/or originate from the cytoplasm.

There is growing evidence that bacterial pathogens have developed strategies to enhance entry into and survival within phagocytic cells, thereby bypassing normal host killing responses (Kang et al., 2005). The net result is a phagosome that shows reduced fusion to late endocytotic compartments. The specific molecular mechanisms involved in modifying phagosome maturation are unknown and may differ for gram-positive and gram-negative bacteria. Therefore, as well as modulating the recruitment and function of leukocytes, galectin-3 may contribute to the overall outcome of bacterial invasion by modulating phagosome maturation and bacterial survival. Further studies need to be performed to test this hypothesis.

The results from this study would suggest that galectin-3 plays a lesser role in the host response to E. coli pneumonia. Sato et al. demonstrated that galectin-3 concentration in the lung is increased in mice following $S$. pn but not $E$. coli infection and that this upregulation of galectin-3 correlates with the onset of neutrophil extravasation (Sato et al., 2002). However it was not reported whether galectin-3 positive mice developed less severe injury in response to E. coli. It is important to note that the severity and progression of pneumonia in the study detailed in this chapter was less marked in wild type mice receiving $E$. coli compared to $S$. pn which makes direct comparisons difficult. The finding that galectin- $3^{-/-}$mice did not develop worse pneumonia in response to E. coli may be a reflection of the E. coli being a less severe model. Infections involving gram-negative bacteria, including $E$. coli, are accompanied by the accumulation of LPS. LPS has been shown to decrease galectin-3 expression in vitro (Liu et al., 1995; Sato and Hughes, 1994). However, galectin-3 binds to LPS of the gram-negative E. coli and such recognition may mediate host cell-pathogen interactions (Mey et al., 1996). Future studies using a higher inoculation or a more virulent strain of $E$. coli are required before it could be construed that galectin-3 does not play a role in E. coli pneumonia.

Interaction of pathogens with host cells and substances is an important step in microbial pathogenesis. Specific recognition of bacterial carbohydrates by host lectins may also be important. The capsules of some gram-positive bacteria, for 
example type XIV $S$. $p n$, have evolved glycoconjugates that contain $\beta$-galactosides and it is proposed that lectins including galectin-3 can recognise these polylactosamine containing glycoconjugates on pathogens (Mandrell et al., 1994; Sato and Nieminen, 2004). Galectin-3 may also bind to type III $S$. pn through a similar mechanism.

In summary, this study has shown that galectin-3 protects against pneumococcal pneumonia through a variety of mechanisms including modulation of immune cell function and exhibiting direct antimicrobial activity. The increased expression of galectin-3 in the lungs of mice following $S$. pn would be sufficient to account for an antimicrobial effect of galectin-3 playing an important role. A schematic representation showing how galectin-3 may influence the clearance of $S$. $p n$ is shown in figure 6.14. In wild type mice, administration of $S$. $p n$ results in the secretion of galectin-3 from resident alveolar macrophages. Secreted galectin-3 has a direct antimicrobial effect on $S$. $p n$ and can modulate immune cell function. Phagocytosis of bacteria by wild type macrophages results in the production of NO helping to restrain bacterial growth. Secreted galectin-3 activates recruited neutrophils resulting in increased phagocytosis of bacteria and the production of ROS, adding to the bactericidal activity of neutrophils. Once phagocytosis has taken place, the neutrophil undergoes apoptosis. Wild type macrophages efficiently phagocytose apoptotic cells, a crucial step for the resolution of inflammation and protection of lung tissues against the immunogenic components of dying cells (figure $6.14 \mathrm{~A})$.

Administration of $S$. $p n$ to a galectin- $3^{-/-}$mouse causes greater damage than to a wild type mouse because many of the above anti-bacterial steps are hindered through a lack of galectin-3. Galectin- $3^{-/-}$macrophages still produce NO following phagocytosis of bacteria, however the amount secreted is significantly less than a wild type macrophage. Recruited neutrophils are activated less, produce less ROS and phagocytose fewer bacteria compared to neutrophils in the lungs of a galectin-3 positive mouse. Galectin- $3^{-/-}$neutrophils may apoptose more readily than wild type neutrophils resulting in an accumulation of dying cells in the lung and these apoptotic cells may not be cleared as efficiently by galectin- $3^{-/-}$macrophages. The 
damage these apoptotic cells have on the lung tissue enables the bacteria to enter the blood stream resulting in sepsis (figure 6.14B).

Alveolar macrophages express and secrete galectin-3 and it is believed that macrophage-derived galectin-3 is crucial for the recovery from a pneumococcal infection. This research has revealed an interesting therapeutic strategy for improving clinical outcomes following infection. It is hoped that intratracheal administration of galectin-3 gene-modified macrophages into lungs will limit the severity of pneumococcal pneumonia. Future work would therefore exploit genetherapy based strategies to treat pneumococcal pneumonia. 
Figure 6.14 - Proposed model of pneumococcal pneumonia infection.

A) Wild type

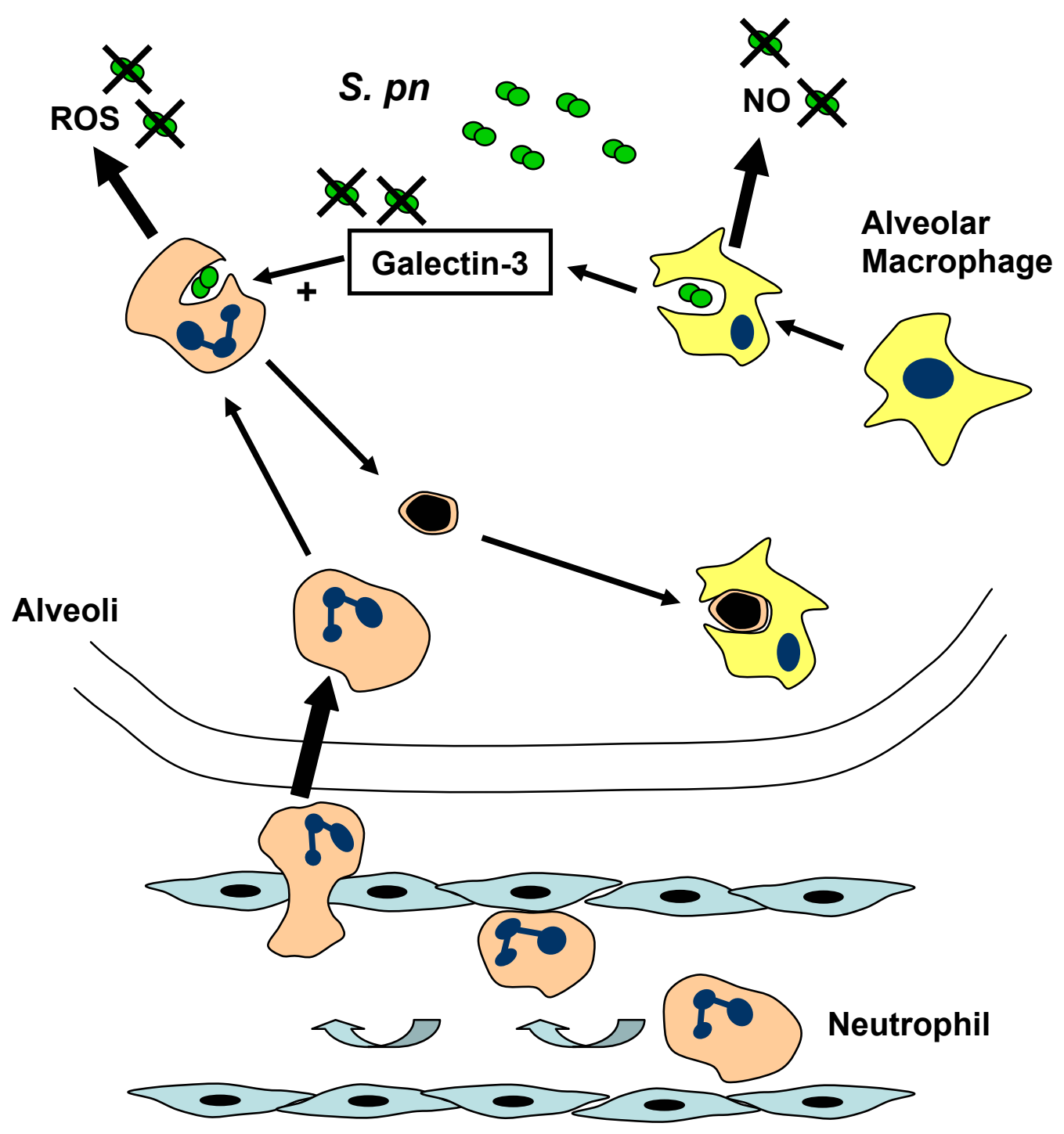

Figure 6.14 (A)

(A) Proposed model of pneumococcal pneumonia infection in wild type mice. Macrophages release galectin-3 and nitric oxide (NO) following phagocytosis of $S$. pn. Galectin-3 accumulates in the alveolar space and together with NO helps to kill the bacteria directly. Galectin-3 activates recruited neutrophils to produce reactive oxygen species (ROS) and enhances their phagocytosis of bacteria. Neutrophils that have eaten bacteria undergo apoptosis and are phagocytosed by macrophages, a key process in the resolution of inflammation which limits the degree of tissue injury. 


\section{B) Galectin-3--}

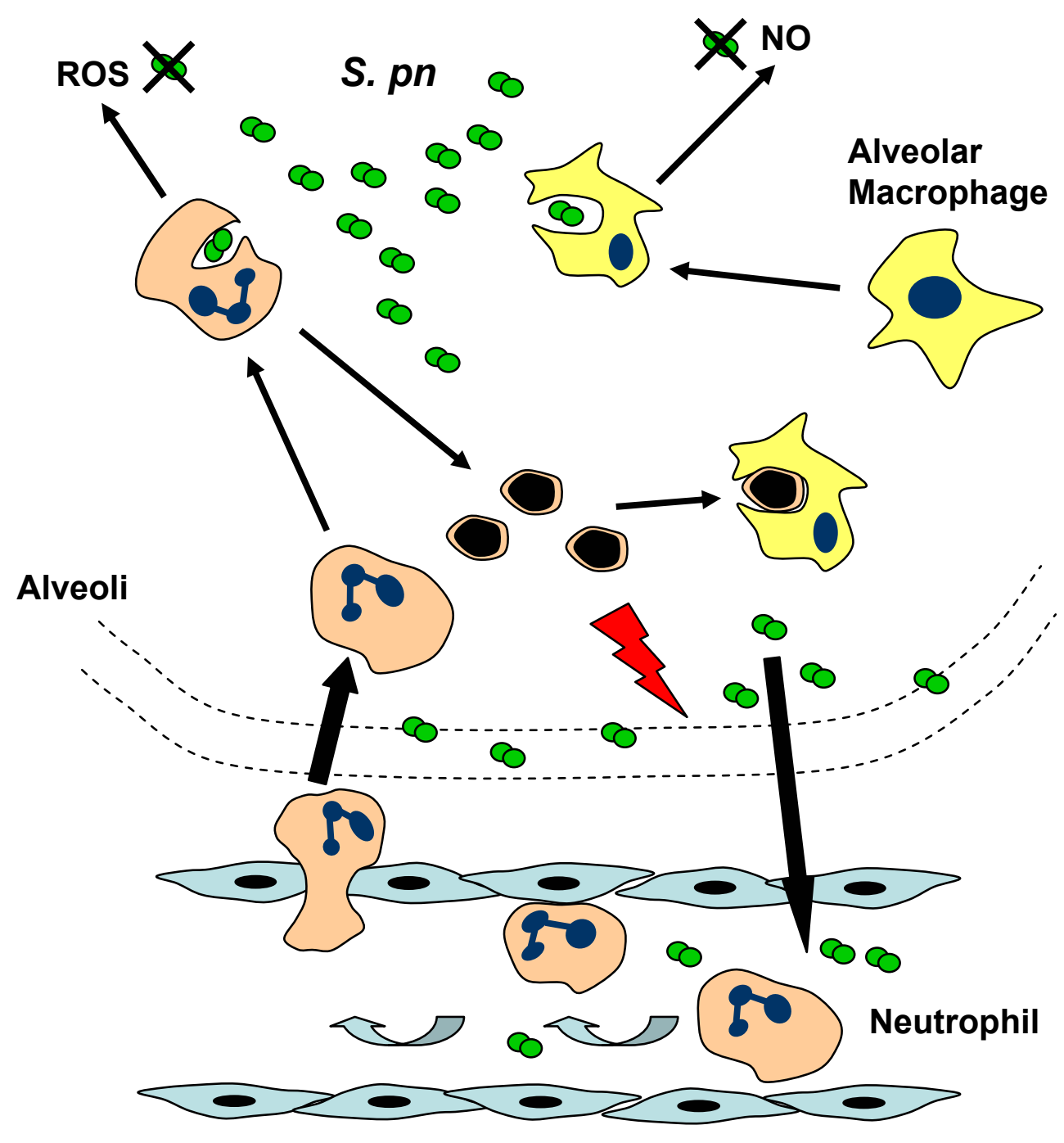

Figure 6.14 (B)

(B) Proposed model of pneumococcal pneumonia infection in galectin- $-3^{-/-}$mice. Galectin- $3^{-/-}$macrophages release less NO following phagocytosis of $S$. pn. The absence of galectin-3 causes reduced activation of neutrophils and consequently reduced production of ROS and phagocytosis of bacteria compared to the galectin-3-activated neutrophils in the wild type mouse. Galectin- $3^{-/-}$neutrophils are more prone to ungergo apoptosis. Galectin- $3^{-/-}$macrophages demonstrate reduced phagocytic activity of apoptotic neutrophils. Greater numbers of histotoxic apoptotic neutrophils damages the lung tissue enabling bacteria to enter the blood stream causing septicemia. 


\section{Chapter 7}

\section{Final conclusions and future directions}

Galectin-3 plays many roles during an immune response. Following an infection or chronic tissue injury, galectin-3 is secreted by resident macrophages where it subsequently functions as a paracrine and autocrine molecule. Extracellular galectin-3 binds to cell surface $\beta$-galactosides containing glycans via the carbohydrate recognition domain (CRD). One of these receptors is CD98, a disulfide-linked $125 \mathrm{kDa}$ heterodimeric type II transmembrane glycoprotein, promoting its dimerisation and, consequently, integrin activation (Dong and Hughes, 1997; Hughes, 2001; Dong and Hughes, 1996; Henderson et al., 2004). The majority of extracellular functions of galectin-3 are thought to be due to the galectin forming oligomers via its $\mathrm{N}$-terminal domain after ligand binding by the $\mathrm{C}$-terminal domain. This interaction creates a molecular lattice on the cell surface important for various biological activities of galectin-3 (Nieminen et al., 2006). Extracellular functions include the modulation of cell-substrate or cell-cell adhesion, cell migration, cell activation, proliferation and survival. In this way, galectin-3 is an important factor during the immune response, cancer progression, chronic inflammation and infection.

I have demonstrated that galectin-3 promotes the progression of fibrosis in multiple organs, including the liver and kidney. Research carried out in this laboratory has also shown that lung fibrosis is perpetuated by the presence of galectin-3 (unpublished finding). Initial findings demonstrated increased galectin-3 expression in established human liver fibrosis with spatial and temporal relationship to fibrosis in a reversible rat model of liver fibrosis. Following these findings, chronic liver and kidney fibrosis was induced in wild type and galectin- $3^{-/-}$mice by chronic carbon tetrachloride $\left(\mathrm{CCl}_{4}\right)$ administration or unilateral ureteric obstruction (UUO) respectively. This in vivo study revealed that galectin-3 plays a critical role during both liver and kidney fibrosis, as galectin- $3^{-/-}$mice showed a marked 
reduction in $\alpha$-smooth muscle actin ( $\alpha$-SMA) and collagen production. Subsequent in vitro experiments revealed that galectin-3 is essential for transforming growth factor- $\beta$ (TGF- $\beta$ ) driven myofibroblast activation. However, to investigate whether galectin-3 signalling and induction of myofibroblast activation is dependent upon TGF- $\beta$, future experiments could include TGF- $\beta$ siRNA of HSCs followed by treatment with recombinant galectin-3. Future work would also involve experiments to elucidate the signalling pathways involved in galectin-3-induced myofibroblast activation.

$\beta$-catenin regulates $\alpha$-SMA expression during TGF- $\beta$-induced epithelial to mesenchymal transition (EMT) (Masszi et al., 2004). Galectin-3 has structural similarity to and is a binding partner of $\beta$-catenin. It is therefore proposed that galectin-3 can stabilise $\beta$-catenin and this interaction may potentiate EMT and myofibroblast survival.

RNA interference of galectin-3 in mouse hepatic stellate cells (HSCs) in vitro resulted in inhibition of myofibroblast activation and procollagen (I) expression. Additional work from this laboratory has shown that administration of galectin-3 siRNA via the portal vein in vivo reduced $\mathrm{HSC}$ activation in response to $\mathrm{CCl}_{4}$ (Henderson et al., 2006). In addition, preliminary work by N. Henderson has demonstrated a decrease in in vivo fibrosis following UUO by administration of galectin-3 siRNA via the renal artery (unpublished). Improvements in siRNA design and delivery has resulted in an increasing amount of research being carried out in vivo. Thus, therapeutic use of siRNA as agents capable of attenuating the expression of disease-causing genes holds great potential (Soutschek et al., 2004).

It has been hypothesised that alternative macrophage activation is one of the key steps toward the progression of fibrosis. Stimulation of macrophages with the Th2 cytokines interleukin-4 (IL-4) or IL-13 results in L-arginine metabolism by arginase (figure 1.4). L-ornithine is produced which is a necessary metabolite for the production of proline (a critical amino acid for the synthesis of collagen) thus linking arginase activity to fibrosis (Hesse et al., 2001; Misson et al., 2004; Endo et al., 2003). This is supported by numerous findings including increased arginase expression and activity in the blood and airways of patients with fibrotic diseases 
(Grasemann et al., 2005; Grasemann et al., 2006; Mora et al., 2006) and in animal models of lung fibrosis (Hesse et al., 2001; Endo et al., 2003) and a shift to classical macrophage activation resulting in inducible nitric oxide synthase (NOS2) expression and a reduction of fibrosis severity (Wynn et al., 1995).

Work presented in Chapter 5 of this thesis detailed the initial work carried out to determine whether galectin-3 is involved in alternative macrophage activation. These results suggest that the key mechanism required for activation of an alternative macrophage phenotype is an IL-4-stimulated galectin-3 feed back loop which directly activates CD98 causing sustained phosphatidylinositol 3-kinase (PI3-K) activation. Therefore, in addition to the regulation of myofibroblast activation, galectin-3 alternatively activates macrophages, both of which may contribute to the persistence of fibrosis seen in mice in vivo. Ongoing studies in this laboratory will help to define the precise biological role of galectin-3-induced alternative macrophages in chronic inflammation and fibrosis.

Based on my results, my hypothesis is that alternative macrophages secrete galectin-3 which goes on to enhance fibroblast activation. To further explore this, alternative macrophage activation or classical macrophage activation of wild type macrophages could be triggered by the addition of IL-4 or interferon- $\gamma$ (IFN $\gamma$ )/ lipopolysaccharide (LPS) respectively and galectin-3 secretion measured. $\alpha$-SMA expression and activation of fibroblasts co-cultured with galectin- $3^{-/-}$macrophages or alternative or classically activated wild type macrophages could be assessed. Galectin-3 inhibitors could also be used to block any effect galectin-3 may have on alternative macrophage-induced fibroblast activation.

Macrophage ablation by the administration of diptheria toxin (DT) to CD11bdiptheria toxin receptor (DTR) mice is one way in which ongoing and future in vivo work will identify the role of macrophages during fibrosis. These mice express the human DTR under the control of the CD11b promoter. This system relies on the fact that the mouse DT receptor binds DT poorly compared with the human molecule. Thus, transgenic expression of the human DTR confers sensitivity to DT and permits ablation in vivo when DT is injected. Renal fibrosis following UUO is being examined in CD11b-DTR mice. Preliminary results suggest that obstructed kidneys of macrophage depleted mice demonstrate reduced $\alpha$-SMA and collagen expression 
compared to mice with no macrophage depletion. Moreover, adoptive transfer of wild type macrophages into galectin- $3^{-/-}$mice significantly increases $\alpha$-SMA expression in the kidney in contrast to galectin- $3^{-/-}$macrophages which did not. These results show that galectin- $3^{-/-}$fibroblasts in the kidney have the potential to transdifferentiate into myofibroblasts when activated by wild type macrophages.

A macrophage/neutrophil specific IL-4 receptor- $\alpha$ (IL-4R $\alpha$ ) deficient mouse has been generated to investigate the role of alternative macrophage activation during schistosomiasis (Herbert et al., 2004). This technology could be exploited to conditionally knockout galectin-3 from macrophages and study the affect this has on organ fibrosis.

In addition to galectin-3 playing a role in perpetuating chronic inflammation, I have shown that galectin-3 protects from acute lethal pneumococcal pneumonia through a variety of mechanisms including modulation of immune cell function. In vivo, galectin-3 plays an important role in the clearance of the gram-positive Streptococcal pneumoniae (S. pn) but not in the gram-negative Escherichia coli (E. coli) pneumonia. Galectin- $3^{-/-}$mice demonstrate a clearance defect of $S$. pn with increased septicaemia and a greater extent of lung damage compared to wild type mice. This phenotype is markedly reduced in an E. coli pneumonia infection. Work from Sato et al. showed that galectin-3 accumulates in the lungs of mice infected with $S$. pn but not E. coli and this increase of galectin-3 correlates with the onset of neutrophil extravasation. Findings from the study detailed in this thesis also showed reduced neutrophil recruitment to the alveolar space in the galectin- $3^{-/-}$mouse. These results provide further evidence in supporting the role of galectin-3 in pneumococcal pneumonia.

It is believed that numerous processes involving galectin-3 are occurring in the lung of the wild type mouse to clear pneumococcal pneumonia. Phagocytosis of bacteria is a crucial step towards the clearance of an infection. Galectin- $3^{-/-}$ macrophages and neutrophils phagocytose $S$. pn just as efficiently as wild type however, addition of exogenous galectin-3 activates neutrophils and increases their ability to phagocytose bacteria. Nitric oxide (NO) production from galectin- $3^{-/-}$ macrophages in response to bacteria is significantly reduced compared to wild type 
macrophages, partially explaining the uncontrolled bacterial growth in the null mouse. Furthermore, galectin-3 has antimicrobial properties against $S$. pn. At present, studies are being performed to elucidate the concentration of galectin-3 in lungs of mice infected with $S$. pn. This will determine whether bactericidal concentrations of galectin-3 in the lungs are reached following infection in vivo.

Clearance of potentially toxic apoptotic neutrophils by macrophages results in the release of anti-inflammatory and reparative cytokines such as TGF- $\beta 1$. These steps help to limit the degree of tissue injury. Results indicate that galectin- $3^{-/}$ neutrophils have a tendency to undergo apoptosis earlier than wild type neutrophils, and galectin- $3^{-/-}$macrophages are less able to phagocytose apoptotic neutrophils compared to wild type macrophages. Together, these data explain the increased protein content in bronchoalveolar lavage (BAL) and septicaemia in the null mice due to the presence of greater numbers of tissue damaging apoptotic neutrophils.

These results are consistent with the hypothesis that galectin-3 released from macrophages in response to pneumococcal pneumonia infection can activate neutrophils and enhance their phagocytic capability so aiding the bacterial clearance observed in wild type mice following pneumococcal infection.

Future work would involve expanding my in vitro studies to fully investigate the role of galectin-3 on neutrophil and macrophage activation and function. This study provides some data regarding the role of galectin-3 in neutrophil apoptosis and phagocytosis of apoptotic neutrophils by macrophages, which would be expanded in future studies. Phagosome maturation, an important process in limiting pathogen replication, could be assessed in wild type and galectin- $3^{-/-}$macrophages and neutrophils following phagocytosis of $S$. pn and E. coli using LysoTracker probes which selectively label acidic organelles in live cells, including mature hydrolytic and acidic phagolysosomes.

In vivo studies would involve administration of galectin-3 intratracheally with $S$. $p n$ in galectin- $3^{-/-}$mice in an attempt to reduce the severity of pneumococcal pneumonia in these mice. The dose of galectin-3 to be administered needs to be carefully thought out as a high dose of galectin-3 has been shown to be antimicrobial towards S. pn in vitro. Other in vivo studies would include depleting various macrophage populations in the wild type mouse and reconstituting them with wild 
type or galectin- $3^{-/-}$macrophages followed by inoculation with S. pn. Clodronate would be used to deplete alveolar macrophages and the role of resident or recruited macrophages would be assessed by giving wild type or galectin- $3^{-/-}$macrophages intratracheally or intravenously respectively. In a second approach, macrophage ablation could be carried out by the administration of diptheria toxin to CD11b-DTR mice followed by targeting wild type or galectin- $3^{-/-}$macrophages to the site of infection. A third approach could explore pneumonia infection in the macrophage/neutrophil-specific galectin-3 deficient mouse.

During an initial inflammatory response, for example following an acute infection with pneumococcal pneumonia, galectin-3 plays a beneficial role as it activates immune cells responsible for clearing the infection and recruits more cells to the site of infection. Once the infection is cleared, the immune response is dampened down. However, during a chronic inflammatory response resulting in fibrosis, galectin-3 is detrimental to the individual. We hypothesise that chronic injury results in the enhanced secretion of galectin-3 from macrophages and this perpetuates the persistant paracrine and autocrine activation of fibroblasts and macrophages. The consequence of this is enhanced collagen deposition and scarring. The plasticity of macrophage activation is crucial for the coordinated response to infection. Galectin-3 may function to restrain the functional phenotype of macrophages and permit the development of anti-inflammatory alternatively activated macrophages. Galectin $-3^{-/-}$macrophages are unable to alternatively activate and this may result in a reduced capacity to dampen down inflammation in response to infection. The macrophage specific IL-4R ${ }^{-/-}$mouse develops severe septicaemia following schistosomiasis infection due to unrestrained inflammation and mobilisation of gut flora (Herbert et al., 2004).

I have shown that the presence of galectin-3 is disadvantageous to the resolution of a fibrotic response. However, during a pneumococcal pneumonia infection, the presence of galectin-3 is beneficial, and indeed critical, for the clearance of an infection. Therefore, with regard to therapies, it is important to be able to specifically target galectin-3 to the site of an infection, for example by administering galectin-3 gene-modified macrophages. Following treatment it is 
essential to be able to control the levels of galectin-3 so that once an infection is cleared, the galectin-3 will not cause fibrosis which could be potentially more fatal than the initial infection. Conversely, with regard to treating fibrotic injury with, for example, galectin-3 inhibitors or using RNA interference techniques, these need to be targeted to specific tissues/cell types, so that it does not result in an increased susceptibility to bacterial infections.

In conclusion, I have shown that galectin-3 plays a critical role in a variety of inflammatory disorders including fibrosis following chronic tissue injury in the liver and kidney and resolution of pneumococcal pneumonia infection, and may therefore present a new avenue for therapeutic strategies. 
Reference List

Acosta-Rodriguez,E.V., Montes,C.L., Motran,C.C., Zuniga,E.I., Liu,F.T., Rabinovich,G.A., and Gruppi,A. (2004). Galectin-3 mediates IL-4-induced survival and differentiation of $\mathrm{B}$ cells: functional cross-talk and implications during Trypanosoma cruzi infection. J. Immunol. 172, 493-502.

Agrwal,N., Wang,J.L., and Voss,P.G. (1989). Carbohydrate-binding protein 35. Levels of transcription and mRNA accumulation in quiescent and proliferating cells. J. Biol. Chem. 264, 17236-17242.

Ahmad,N., Gabius,H.J., Andre,S., Kaltner,H., Sabesan,S., Roy,R., Liu,B., Macaluso,F., and Brewer,C.F. (2004). Galectin-3 precipitates as a pentamer with synthetic multivalent carbohydrates and forms heterogeneous cross-linked complexes. J. Biol. Chem. 279, 10841-10847.

Akahani,S., Nangia-Makker,P., Inohara,H., Kim,H.R., and Raz,A. (1997). Galectin3: a novel antiapoptotic molecule with a functional BH1 (NWGR) domain of Bcl-2 family. Cancer Res. 57, 5272-5276.

Alspaugh,J.A. and Granger,D.L. (1991). Inhibition of Cryptococcus neoformans replication by nitrogen oxides supports the role of these molecules as effectors of macrophage-mediated cytostasis. Infect. Immun. 59, 2291-2296.

Andre,S., Kojima,S., Yamazaki,N., Fink,C., Kaltner,H., Kayser,K., and Gabius,H.J. (1999). Galectins-1 and -3 and their ligands in tumor biology. Non-uniform properties in cell-surface presentation and modulation of adhesion to matrix glycoproteins for various tumor cell lines, in biodistribution of free and liposomebound galectins and in their expression by breast and colorectal carcinomas with/without metastatic propensity. J. Cancer Res. Clin. Oncol. 125, 461-474.

Asseffa,A., Dickson,L.A., Mohla,S., and Bremner,T.A. (1993). Phorbol myristate acetate-differentiated THP-1 cells display increased levels of MHC class I and class II mRNA and interferon-gamma-inducible tumoricidal activity. Oncol. Res. 5, 11-18.

Bachhawat-Sikder,K., Thomas,C.J., and Surolia,A. (2001). Thermodynamic analysis of the binding of galactose and poly-N-acetyllactosamine derivatives to human galectin-3. FEBS Lett. 500, 75-79.

Baldus,S.E., Zirbes,T.K., Weingarten,M., Fromm,S., Glossmann,J., Hanisch,F.G., Monig,S.P., Schroder,W., Flucke,U., Thiele,J., Holscher,A.H., and Dienes,H.P. (2000). Increased galectin-3 expression in gastric cancer: correlations with histopathological subtypes, galactosylated antigens and tumor cell proliferation. Tumour. Biol. 21, 258-266. 
Bao,Q. and Hughes,R.C. (1995). Galectin-3 expression and effects on cyst enlargement and tubulogenesis in kidney epithelial MDCK cells cultured in threedimensional matrices in vitro. J. Cell Sci. 108 ( Pt 8), 2791-2800.

Barendsen,N., Mueller,M., and Chen,B. (1990). Inhibition of TPA-induced monocytic differentiation in THP-1 human monocytic leukemic cells by staurosporine, a potent protein kinase C inhibitor. Leuk. Res. 14, 467-474.

Barondes,S.H., Castronovo,V., Cooper,D.N., Cummings,R.D., Drickamer,K., Feizi,T., Gitt,M.A., Hirabayashi,J., Hughes,C., Kasai,K., and . (1994a). Galectins: a family of animal beta-galactoside-binding lectins. Cell 76, 597-598.

Barondes,S.H., Cooper,D.N., Gitt,M.A., and Leffler,H. (1994b). Galectins. Structure and function of a large family of animal lectins. J. Biol. Chem. 269, 20807-20810.

Bataller,R. and Brenner,D.A. (2005). Liver fibrosis. J. Clin. Invest 115, 209-218.

Beatty,W.L., Rhoades,E.R., Hsu,D.K., Liu,F.T., and Russell,D.G. (2002).

Association of a macrophage galactoside-binding protein with Mycobacteriumcontaining phagosomes. Cell Microbiol. 4, 167-176.

Bergeron,Y., Ouellet,N., Deslauriers,A.M., Simard,M., Olivier,M., and Bergeron,M.G. (1998). Cytokine kinetics and other host factors in response to pneumococcal pulmonary infection in mice. Infect. Immun. 66, 912-922.

Bi,Z. and Reiss,C.S. (1995). Inhibition of vesicular stomatitis virus infection by nitric oxide. J. Virol. 69, 2208-2213.

Bonis,P.A., Friedman,S.L., and Kaplan,M.M. (2001). Is liver fibrosis reversible? N. Engl. J. Med. 344, 452-454.

Boxio,R., Bossenmeyer-Pourie,C., Steinckwich,N., Dournon,C., and Nusse,O. (2004). Mouse bone marrow contains large numbers of functionally competent neutrophils. J. Leukoc. Biol. 75, 604-611.

Bresalier,R.S., Yan,P.S., Byrd,J.C., Lotan,R., and Raz,A. (1997). Expression of the endogenous galactose-binding protein galectin-3 correlates with the malignant potential of tumors in the central nervous system. Cancer 80, 776-787.

Brown,P.D. and Lerner,S.A. (1998). Community-acquired pneumonia. Lancet 352, 1295-1302.

Bucala,R., Spiegel,L.A., Chesney,J., Hogan,M., and Cerami,A. (1994). Circulating fibrocytes define a new leukocyte subpopulation that mediates tissue repair. Mol. Med. 1, 71-81.

Buttery,R., Monaghan,H., Salter,D.M., and Sethi,T. (2004). Galectin-3: differential expression between small-cell and non-small-cell lung cancer. Histopathology 44, 339-344. 
Cailhier,J.F., Partolina,M., Vuthoori,S., Wu,S., Ko,K., Watson,S., Savill,J., Hughes,J., and Lang,R.A. (2005). Conditional macrophage ablation demonstrates that resident macrophages initiate acute peritoneal inflammation. J. Immunol. 174, 2336-2342.

Califice,S., Castronovo,V., Bracke,M., and van den,B.F. (2004). Dual activities of galectin-3 in human prostate cancer: tumor suppression of nuclear galectin-3 vs tumor promotion of cytoplasmic galectin-3. Oncogene 23, 7527-7536.

Castronovo,V., Campo,E., Van Den Brule,F.A., Claysmith,A.P., Cioce,V., Liu,F.T., Fernandez,P.L., and Sobel,M.E. (1992). Inverse modulation of steady-state messenger RNA levels of two non-integrin laminin-binding proteins in human colon carcinoma. J. Natl. Cancer Inst. 84, 1161-1169.

Castronovo,V., Van Den Brule,F.A., Jackers,P., Clausse,N., Liu,F.T., Gillet,C., and Sobel,M.E. (1996). Decreased expression of galectin-3 is associated with progression of human breast cancer. J. Pathol. 179, 43-48.

Chen,H.Y., Liu,F.T., and Yang,R.Y. (2005). Roles of galectin-3 in immune responses. Arch. Immunol. Ther. Exp. (Warsz. ) 53, 497-504.

Chiaramonte,M.G., Cheever,A.W., Malley,J.D., Donaldson,D.D., and Wynn,T.A. (2001). Studies of murine schistosomiasis reveal interleukin-13 blockade as a treatment for established and progressive liver fibrosis. Hepatology 34, 273-282.

Chiariotti,L., Salvatore,P., Frunzio,R., and Bruni,C.B. (2004). Galectin genes: regulation of expression. Glycoconj. J. 19, 441-449.

Choufani,G., Nagy,N., Saussez,S., Marchant,H., Bisschop,P., Burchert,M., Danguy,A., Louryan,S., Salmon,I., Gabius,H.J., Kiss,R., and Hassid,S. (1999). The levels of expression of galectin-1, galectin-3, and the Thomsen-Friedenreich antigen and their binding sites decrease as clinical aggressiveness increases in head and neck cancers. Cancer 86, 2353-2363.

Cindolo,L., Benvenuto,G., Salvatore,P., Pero,R., Salvatore,G., Mirone,V., Prezioso,D., Altieri,V., Bruni,C.B., and Chiariotti,L. (1999). galectin-1 and galectin3 expression in human bladder transitional-cell carcinomas. Int. J. Cancer 84, 39-43.

Colnot,C., Fowlis,D., Ripoche,M.A., Bouchaert,I., and Poirier,F. (1998a). Embryonic implantation in galectin 1/galectin 3 double mutant mice. Dev. Dyn. 211, 306-313.

Colnot,C., Ripoche,M.A., Milon,G., Montagutelli,X., Crocker,P.R., and Poirier,F. (1998b). Maintenance of granulocyte numbers during acute peritonitis is defective in galectin-3-null mutant mice. Immunology 94, 290-296.

Corraliza,I.M., Campo,M.L., Soler,G., and Modolell,M. (1994). Determination of arginase activity in macrophages: a micromethod. J. Immunol. Methods 174, 231235. 
Cortegano,I., del,P., V, Cardaba,B., de Andres,B., Gallardo,S., del Amo,A., Arrieta,I., Jurado,A., Palomino,P., Liu,F.T., and Lahoz,C. (1998). Galectin-3 downregulates IL-5 gene expression on different cell types. J. Immunol. 161, 385-389.

Cumpstey,I., Sundin,A., Leffler,H., and Nilsson,U.J. (2005). C2-symmetrical thiodigalactoside bis-benzamido derivatives as high-affinity inhibitors of galectin-3: efficient lectin inhibition through double arginine-arene interactions. Angew. Chem. Int. Ed Engl. 44, 5110-5112.

D'Andrea,A., Ma,X., Aste-Amezaga,M., Paganin,C., and Trinchieri,G. (1995).

Stimulatory and inhibitory effects of interleukin (IL)-4 and IL-13 on the production of cytokines by human peripheral blood mononuclear cells: priming for IL-12 and tumor necrosis factor alpha production. J. Exp. Med. 181, 537-546.

Dabelic,S., Supraha,S., and Dumic,J. (2005). Galectin-3 in macrophage-like cells exposed to immunomodulatory drugs. Biochim. Biophys. Acta.

Dagher,S.F., Wang,J.L., and Patterson,R.J. (1995). Identification of galectin-3 as a factor in pre-mRNA splicing. Proc. Natl. Acad. Sci. U. S. A 92, 1213-1217.

Dahlgren,C. and Karlsson,A. (1999). Respiratory burst in human neutrophils. J. Immunol. Methods 232, 3-14.

Dallaire,F., Ouellet,N., Bergeron,Y., Turmel,V., Gauthier,M.C., Simard,M., and Bergeron,M.G. (2001). Microbiological and inflammatory factors associated with the development of pneumococcal pneumonia. J. Infect. Dis. 184, 292-300.

Davidson,P.J., Davis,M.J., Patterson,R.J., Ripoche,M.A., Poirier,F., and Wang,J.L. (2002). Shuttling of galectin-3 between the nucleus and cytoplasm. Glycobiology 12, 329-337.

de Waard,A., Hickman,S., and Kornfeld,S. (1976). Isolation and properties of betagalactoside binding lectins of calf heart and lung. J. Biol. Chem. 251, 7581-7587.

del,P., V, Rojo,M., Rubio,M.L., Cortegano,I., Cardaba,B., Gallardo,S., Ortega,M., Civantos,E., Lopez,E., Martin-Mosquero,C., Peces-Barba,G., Palomino,P., Gonzalez-Mangado,N., and Lahoz,C. (2002). Gene therapy with galectin-3 inhibits bronchial obstruction and inflammation in antigen-challenged rats through interleukin-5 gene downregulation. Am. J. Respir. Crit Care Med. 166, 732-737.

Delacour,D., Greb,C., Koch,A., Salomonsson,E., Leffler,H., Le Bivic,A., and Jacob,R. (2007). Apical sorting by galectin-3-dependent glycoprotein clustering. Traffic. 8, 379-388.

Delacour,D. and Jacob,R. (2006). Apical protein transport. Cell Mol. Life Sci. 63, 2491-2505.

Demetriou,M., Granovsky,M., Quaggin,S., and Dennis,J.W. (2001). Negative regulation of T-cell activation and autoimmunity by Mgat $5 \mathrm{~N}$-glycosylation. Nature 409, 733-739. 
Derynck,R. and Zhang,Y.E. (2003). Smad-dependent and Smad-independent pathways in TGF-beta family signalling. Nature 425, 577-584.

Desnues,B., Lepidi,H., Raoult,D., and Mege,J.L. (2005). Whipple disease: intestinal infiltrating cells exhibit a transcriptional pattern of M2/alternatively activated macrophages. J. Infect. Dis. 192, 1642-1646.

Deves,R. and Boyd,C.A. (2000). Surface antigen CD98(4F2): not a single membrane protein, but a family of proteins with multiple functions. J. Membr. Biol. 173, 165177.

Diamond,J.R. (1995). Macrophages and progressive renal disease in experimental hydronephrosis. Am. J. Kidney Dis. 26, 133-140.

Diamond,J.R., van Goor,H., Ding,G., and Engelmyer,E. (1995). Myofibroblasts in experimental hydronephrosis. Am. J. Pathol. 146, 121-129.

Diaz-Guerra,M.J., Castrillo,A., Martin-Sanz,P., and Bosca,L. (1999). Negative regulation by phosphatidylinositol 3-kinase of inducible nitric oxide synthase expression in macrophages. J. Immunol. 162, 6184-6190.

Dietz,A.B., Bulur,P.A., Knutson,G.J., Matasic,R., and Vuk-Pavlovic,S. (2000). Maturation of human monocyte-derived dendritic cells studied by microarray hybridization. Biochem. Biophys. Res. Commun. 275, 731-738.

Dockrell,D.H., Marriott,H.M., Prince,L.R., Ridger,V.C., Ince,P.G., Hellewell,P.G., and Whyte,M.K. (2003). Alveolar macrophage apoptosis contributes to pneumococcal clearance in a resolving model of pulmonary infection. J. Immunol. $171,5380-5388$.

Dong,S. and Hughes,R.C. (1996). Galectin-3 stimulates uptake of extracellular Ca2+ in human Jurkat T-cells. FEBS Lett. 395, 165-169.

Dong,S. and Hughes,R.C. (1997). Macrophage surface glycoproteins binding to galectin-3 (Mac-2-antigen). Glycoconj. J. 14, 267-274.

Donnelly,S., O'Neill,S.M., Sekiya,M., Mulcahy,G., and Dalton,J.P. (2005).

Thioredoxin peroxidase secreted by Fasciola hepatica induces the alternative activation of macrophages. Infect. Immun. 73, 166-173.

Dransfield,I., Buckle,A.M., Savill,J.S., McDowall,A., Haslett,C., and Hogg,N. (1994). Neutrophil apoptosis is associated with a reduction in CD16 (Fc gamma RIII) expression. J. Immunol. 153, 1254-1263.

Duffield,J.S., Forbes,S.J., Constandinou,C.M., Clay,S., Partolina,M., Vuthoori,S., Wu,S., Lang,R., and Iredale,J.P. (2005a). Selective depletion of macrophages reveals distinct, opposing roles during liver injury and repair. J. Clin. Invest 115, 56-65. 
Duffield,J.S., Tipping,P.G., Kipari,T., Cailhier,J.F., Clay,S., Lang,R., Bonventre,J.V., and Hughes,J. (2005b). Conditional ablation of macrophages halts progression of crescentic glomerulonephritis. Am. J. Pathol. 167, 1207-1219.

Dumic,J., Dabelic,S., and Flogel,M. (2006). Galectin-3: An open-ended story. Biochim. Biophys. Acta 1760, 616-635.

Dumic,J., Lauc,G., and Flogel,M. (2000). Expression of galectin-3 in cells exposed to stress-roles of jun and NF-kappaB. Cell Physiol Biochem. 10, 149-158.

Elad-Sfadia,G., Haklai,R., Balan,E., and Kloog,Y. (2004). Galectin-3 augments KRas activation and triggers a Ras signal that attenuates ERK but not phosphoinositide 3-kinase activity. J. Biol. Chem. 279, 34922-34930.

Ellerhorst,J., Troncoso,P., Xu,X.C., Lee,J., and Lotan,R. (1999). Galectin-1 and galectin-3 expression in human prostate tissue and prostate cancer. Urol. Res. 27, 362-367.

Elsharkawy,A.M., Oakley,F., and Mann,D.A. (2005). The role and regulation of hepatic stellate cell apoptosis in reversal of liver fibrosis. Apoptosis. 10, 927-939.

Endo,M., Oyadomari,S., Terasaki,Y., Takeya,M., Suga,M., Mori,M., and Gotoh,T. (2003). Induction of arginase I and II in bleomycin-induced fibrosis of mouse lung. Am. J. Physiol Lung Cell Mol. Physiol 285, L313-L321.

Feldman,C. (2004). Clinical relevance of antimicrobial resistance in the management of pneumococcal community-acquired pneumonia. J. Lab Clin. Med. 143, 269-283.

Fernandez,G.C., Ilarregui,J.M., Rubel,C.J., Toscano,M.A., Gomez,S.A., Beigier,B.M., Isturiz,M.A., Rabinovich,G.A., and Palermo,M.S. (2005). Galectin-3 and soluble fibrinogen act in concert to modulate neutrophil activation and survival: involvement of alternative MAPK pathways. Glycobiology 15, 519-527.

Feuk-Lagerstedt,E., Jordan,E.T., Leffler,H., Dahlgren,C., and Karlsson,A. (1999). Identification of CD66a and CD66b as the major galectin-3 receptor candidates in human neutrophils. J. Immunol. 163, 5592-5598.

Franke-Ullmann,G., Pfortner,C., Walter,P., Steinmuller,C., Lohmann-Matthes,M.L., and Kobzik,L. (1996). Characterization of murine lung interstitial macrophages in comparison with alveolar macrophages in vitro. J. Immunol. 157, 3097-3104.

Friedman,S.L. (2000). Molecular regulation of hepatic fibrosis, an integrated cellular response to tissue injury. J. Biol. Chem. 275, 2247-2250.

Frigeri,L.G., Zuberi,R.I., and Liu,F.T. (1993). Epsilon BP, a beta-galactoside-binding animal lectin, recognizes $\mathrm{IgE}$ receptor ( $\mathrm{Fc}$ epsilon $\mathrm{RI}$ ) and activates mast cells.

Biochemistry 32, 7644-7649. 
Fukumori,T., Takenaka,Y., Oka,N., Yoshii,T., Hogan,V., Inohara,H., Kanayama,H.O., Kim,H.R., and Raz,A. (2004). Endogenous galectin-3 determines the routing of CD95 apoptotic signaling pathways. Cancer Res. 64, 3376-3379.

Fukumori,T., Takenaka,Y., Yoshii,T., Kim,H.R., Hogan,V., Inohara,H., Kagawa,S., and Raz,A. (2003). CD29 and CD7 mediate galectin-3-induced type II T-cell apoptosis. Cancer Res. 63, 8302-8311.

Fukushi,J., Makagiansar,I.T., and Stallcup,W.B. (2004). NG2 proteoglycan promotes endothelial cell motility and angiogenesis via engagement of galectin-3 and alpha3beta1 integrin. Mol. Biol. Cell 15, 3580-3590.

Garin,J., Diez,R., Kieffer,S., Dermine,J.F., Duclos,S., Gagnon,E., Sadoul,R., Rondeau,C., and Desjardins,M. (2001). The phagosome proteome: insight into phagosome functions. J. Cell Biol. 152, 165-180.

Glinsky,V.V., Glinsky,G.V., Rittenhouse-Olson,K., Huflejt,M.E., Glinskii,O.V., Deutscher,S.L., and Quinn,T.P. (2001). The role of Thomsen-Friedenreich antigen in adhesion of human breast and prostate cancer cells to the endothelium. Cancer Res. $61,4851-4857$.

Goletz,S., Hanisch,F.G., and Karsten,U. (1997). Novel alphaGalNAc containing glycans on cytokeratins are recognized invitro by galectins with type II carbohydrate recognition domains. J. Cell Sci. 110 ( Pt 14), 1585-1596.

Gong,H.C., Honjo,Y., Nangia-Makker,P., Hogan,V., Mazurak,N., Bresalier,R.S., and Raz,A. (1999). The NH2 terminus of galectin-3 governs cellular compartmentalization and functions in cancer cells. Cancer Res. 59, 6239-6245.

Gordon,S. (2003). Alternative activation of macrophages. Nat. Rev. Immunol. 3, 23 35.

Gordon,S., Fraser,I., Nath,D., Hughes,D., and Clarke,S. (1992). Macrophages in tissues and in vitro. Curr. Opin. Immunol. 4, 25-32.

Gordon,S.B., Irving,G.R., Lawson,R.A., Lee,M.E., and Read,R.C. (2000).

Intracellular trafficking and killing of Streptococcus pneumoniae by human alveolar macrophages are influenced by opsonins. Infect. Immun. 68, 2286-2293.

Grasemann,H., Schwiertz,R., Grasemann,C., Vester,U., Racke,K., and Ratjen,F. (2006). Decreased systemic bioavailability of L-arginine in patients with cystic fibrosis. Respir. Res. 7, 87.

Grasemann,H., Schwiertz,R., Matthiesen,S., Racke,K., and Ratjen,F. (2005).

Increased arginase activity in cystic fibrosis airways. Am. J. Respir. Crit Care Med. $172,1523-1528$.

Griess P. Ber.Deutsch.Chem.Ges 12, 426. 1879.

Ref Type: Generic 
Gritzmacher,C.A., Mehl,V.S., and Liu,F.T. (1992). Genomic cloning of the gene for an IgE-binding lectin reveals unusual utilization of 5 ' untranslated regions.

Biochemistry 31, 9533-9538.

Hauschildt,S., Bassenge,E., Bessler,W., Busse,R., and Mulsch,A. (1990). L-argininedependent nitric oxide formation and nitrite release in bone marrow-derived macrophages stimulated with bacterial lipopeptide and lipopolysaccharide. Immunology 70, 332-337.

Hayashi,H., Abdollah,S., Qiu,Y., Cai,J., Xu,Y.Y., Grinnell,B.W., Richardson,M.A., Topper,J.N., Gimbrone,M.A., Jr., Wrana,J.L., and Falb,D. (1997). The MAD-related protein Smad7 associates with the TGFbeta receptor and functions as an antagonist of TGFbeta signaling. Cell 89, 1165-1173.

Haynes,B.F., Hemler,M.E., Mann,D.L., Eisenbarth,G.S., Shelhamer,J., Mostowski,H.S., Thomas,C.A., Strominger,J.L., and Fauci,A.S. (1981).

Characterization of a monoclonal antibody (4F2) that binds to human monocytes and to a subset of activated lymphocytes. J. Immunol. 126, 1409-1414.

Hebenstreit,D., Wirnsberger,G., Horejs-Hoeck,J., and Duschl,A. (2006). Signaling mechanisms, interaction partners, and target genes of STAT6. Cytokine Growth Factor Rev. 17, 173-188.

Heldin,C.H., Miyazono,K., and ten Dijke,P. (1997). TGF-beta signalling from cell membrane to nucleus through SMAD proteins. Nature 390, 465-471.

Heldin,C.H. and Westermark,B. (1999). Mechanism of action and in vivo role of platelet-derived growth factor. Physiol Rev. 79, 1283-1316.

Henderson,N.C., Collis,E.A., Mackinnon,A.C., Simpson,K.J., Haslett,C., Zent,R., Ginsberg,M., and Sethi,T. (2004). CD98hc (SLC3A2) interaction with beta 1 integrins is required for transformation. J. Biol. Chem. 279, 54731-54741.

Henderson,N.C., Mackinnon,A.C., Farnworth,S.L., Poirier,F., Russo,F.P., Iredale,J.P., Haslett,C., Simpson,K.J., and Sethi,T. (2006). Galectin-3 regulates myofibroblast activation and hepatic fibrosis. Proc. Natl. Acad. Sci. U. S. A 103, 5060-5065.

Herbert,D.R., Holscher,C., Mohrs,M., Arendse,B., Schwegmann,A., Radwanska,M., Leeto,M., Kirsch,R., Hall,P., Mossmann,H., Claussen,B., Forster,I., and Brombacher,F. (2004). Alternative macrophage activation is essential for survival during schistosomiasis and downmodulates $\mathrm{T}$ helper 1 responses and immunopathology. Immunity. 20, 623-635.

Hesse,M., Modolell,M., La Flamme,A.C., Schito,M., Fuentes,J.M., Cheever,A.W., Pearce,E.J., and Wynn,T.A. (2001). Differential regulation of nitric oxide synthase-2 and arginase- 1 by type 1/type 2 cytokines in vivo: granulomatous pathology is shaped by the pattern of L-arginine metabolism. J. Immunol. 167, 6533-6544. 
Hikita,C., Vijayakumar,S., Takito,J., Erdjument-Bromage,H., Tempst,P., and Al Awqati,Q. (2000). Induction of terminal differentiation in epithelial cells requires polymerization of hensin by galectin 3. J. Cell Biol. 151, 1235-1246.

Hinz,B. (2007). Formation and function of the myofibroblast during tissue repair. J. Invest Dermatol. 127, 526-537.

Hinz,B., Phan,S.H., Thannickal,V.J., Galli,A., Bochaton-Piallat,M.L., and Gabbiani,G. (2007). The Myofibroblast. One Function, Multiple Origins. Am. J. Pathol.

Hirabayashi,J. and Kasai,K. (1993). The family of metazoan metal-independent betagalactoside-binding lectins: structure, function and molecular evolution. Glycobiology 3, 297-304.

Ho,M.K. and Springer,T.A. (1982). Mac-2, a novel 32,000 Mr mouse macrophage subpopulation-specific antigen defined by monoclonal antibodies. J. Immunol. 128, 1221-1228.

Hogg,J.C. and Doerschuk,C.M. (1995). Leukocyte traffic in the lung. Annu. Rev. Physiol 57, 97-114.

Holscher,C., Arendse,B., Schwegmann,A., Myburgh,E., and Brombacher,F. (2006). Impairment of alternative macrophage activation delays cutaneous leishmaniasis in nonhealing BALB/c mice. J. Immunol. 176, 1115-1121.

Holscher,C., Atkinson,R.A., Arendse,B., Brown,N., Myburgh,E., Alber,G., and Brombacher,F. (2001). A protective and agonistic function of IL-12p40 in mycobacterial infection. J. Immunol. 167, 6957-6966.

Honjo,Y., Inohara,H., Akahani,S., Yoshii,T., Takenaka,Y., Yoshida,J., Hattori,K., Tomiyama,Y., Raz,A., and Kubo,T. (2000). Expression of cytoplasmic galectin-3 as a prognostic marker in tongue carcinoma. Clin. Cancer Res. 6, 4635-4640.

Honjo,Y., Nangia-Makker,P., Inohara,H., and Raz,A. (2001). Down-regulation of galectin-3 suppresses tumorigenicity of human breast carcinoma cells. Clin. Cancer Res. 7, 661-668.

Hsu,D.K., Dowling,C.A., Jeng,K.C., Chen,J.T., Yang,R.Y., and Liu,F.T. (1999). Galectin-3 expression is induced in cirrhotic liver and hepatocellular carcinoma. Int. J. Cancer 81, 519-526.

Hsu,D.K., Hammes,S.R., Kuwabara,I., Greene,W.C., and Liu,F.T. (1996). Human T lymphotropic virus-I infection of human $\mathrm{T}$ lymphocytes induces expression of the beta-galactoside-binding lectin, galectin-3. Am. J. Pathol. 148, 1661-1670.

Hsu,D.K., Yang,R.Y., Pan,Z., Yu,L., Salomon,D.R., Fung-Leung,W.P., and Liu,F.T. (2000). Targeted disruption of the galectin-3 gene results in attenuated peritoneal inflammatory responses. Am. J. Pathol. 156, 1073-1083. 
Hughes, R.C. (1999). Secretion of the galectin family of mammalian carbohydratebinding proteins. Biochim. Biophys. Acta 1473, 172-185.

Hughes,R.C. (2001). Galectins as modulators of cell adhesion. Biochimie 83, 667676.

Ikeda,K., Wakahara,T., Wang,Y.Q., Kadoya,H., Kawada,N., and Kaneda,K. (1999). In vitro migratory potential of rat quiescent hepatic stellate cells and its augmentation by cell activation. Hepatology 29, 1760-1767.

Inohara,H., Akahani,S., Koths,K., and Raz,A. (1996). Interactions between galectin3 and Mac-2-binding protein mediate cell-cell adhesion. Cancer Res. 56, 4530-4534.

Inohara,H., Akahani,S., and Raz,A. (1998). Galectin-3 stimulates cell proliferation. Exp. Cell Res. 245, 294-302.

Inohara,H. and Raz,A. (1995). Functional evidence that cell surface galectin-3 mediates homotypic cell adhesion. Cancer Res. 55, 3267-3271.

Iredale,J.P., Benyon,R.C., Pickering,J., McCullen,M., Northrop,M., Pawley,S., Hovell,C., and Arthur,M.J. (1998). Mechanisms of spontaneous resolution of rat liver fibrosis. Hepatic stellate cell apoptosis and reduced hepatic expression of metalloproteinase inhibitors. J. Clin. Invest 102, 538-549.

Irimura,T., Matsushita,Y., Sutton,R.C., Carralero,D., Ohannesian,D.W., Cleary,K.R., Ota,D.M., Nicolson,G.L., and Lotan,R. (1991). Increased content of an endogenous lactose-binding lectin in human colorectal carcinoma progressed to metastatic stages. Cancer Res. 51, 387-393.

Issa,R., Zhou,X., Constandinou,C.M., Fallowfield,J., Millward-Sadler,H., Gaca,M.D., Sands,E., Suliman,I., Trim,N., Knorr,A., Arthur,M.J., Benyon,R.C., and Iredale,J.P. (2004). Spontaneous recovery from micronodular cirrhosis: evidence for incomplete resolution associated with matrix cross-linking. Gastroenterology 126, 1795-1808.

Itou,T., Collins,L.V., Thoren,F.B., Dahlgren,C., and Karlsson,A. (2006). Changes in activation states of murine polymorphonuclear leukocytes (PMN) during inflammation: a comparison of bone marrow and peritoneal exudate PMN. Clin. Vaccine Immunol. 13, 575-583.

Iurisci,I., Tinari,N., Natoli,C., Angelucci,D., Cianchetti,E., and Iacobelli,S. (2000). Concentrations of galectin-3 in the sera of normal controls and cancer patients. Clin. Cancer Res. 6, 1389-1393.

Jonsson,S., Musher,D.M., Chapman,A., Goree,A., and Lawrence,E.C. (1985). Phagocytosis and killing of common bacterial pathogens of the lung by human alveolar macrophages. J. Infect. Dis. 152, 4-13. 
Joo,H.G., Goedegebuure,P.S., Sadanaga,N., Nagoshi,M., von Bernstorff,W., and Eberlein,T.J. (2001). Expression and function of galectin-3, a beta-galactosidebinding protein in activated T lymphocytes. J. Leukoc. Biol. 69, 555-564.

Kadrofske,M.M., Openo,K.P., and Wang,J.L. (1998). The human LGALS3 (galectin3 ) gene: determination of the gene structure and functional characterization of the promoter. Arch. Biochem. Biophys. 349, 7-20.

Kahnert,A., Seiler,P., Stein,M., Bandermann,S., Hahnke,K., Mollenkopf,H., and Kaufmann,S.H. (2006). Alternative activation deprives macrophages of a coordinated defense program to Mycobacterium tuberculosis. Eur. J. Immunol. 36, 631-647.

Kalluri,R. and Neilson,E.G. (2003). Epithelial-mesenchymal transition and its implications for fibrosis. J. Clin. Invest 112, 1776-1784.

Kang,P.B., Azad,A.K., Torrelles,J.B., Kaufman,T.M., Beharka,A., Tibesar,E., DesJardin,L.E., and Schlesinger,L.S. (2005). The human macrophage mannose receptor directs Mycobacterium tuberculosis lipoarabinomannan-mediated phagosome biogenesis. J. Exp. Med. 202, 987-999.

Karlsson,A., Follin,P., Leffler,H., and Dahlgren,C. (1998). Galectin-3 activates the NADPH-oxidase in exudated but not peripheral blood neutrophils. Blood 91, 34303438 .

Kasper,M. and Hughes,R.C. (1996). Immunocytochemical evidence for a modulation of galectin 3 (Mac-2), a carbohydrate binding protein, in pulmonary fibrosis. J. Pathol. 179, 309-316.

Kaviratne,M., Hesse,M., Leusink,M., Cheever,A.W., Davies,S.J., McKerrow,J.H., Wakefield,L.M., Letterio,J.J., and Wynn,T.A. (2004). IL-13 activates a mechanism of tissue fibrosis that is completely TGF-beta independent. J. Immunol. 173, 40204029 .

Kim,H.R., Lin,H.M., Biliran,H., and Raz,A. (1999). Cell cycle arrest and inhibition of anoikis by galectin-3 in human breast epithelial cells. Cancer Res. 59, 4148-4154.

Kim,K., Mayer,E.P., and Nachtigal,M. (2003). Galectin-3 expression in macrophages is signaled by Ras/MAP kinase pathway and up-regulated by modified lipoproteins. Biochim. Biophys. Acta 1641, 13-23.

Kipari,T., Cailhier,J.F., Ferenbach,D., Watson,S., Houlberg,K., Walbaum,D., Clay,S., Savill,J., and Hughes,J. (2006). Nitric oxide is an important mediator of renal tubular epithelial cell death in vitro and in murine experimental hydronephrosis. Am. J. Pathol. 169, 388-399.

Klahr,S. and Morrissey,J. (2002). Obstructive nephropathy and renal fibrosis. Am. J. Physiol Renal Physiol 283, F861-F875. 
Kohatsu,L., Hsu,D.K., Jegalian,A.G., Liu,F.T., and Baum,L.G. (2006). Galectin-3 induces death of Candida species expressing specific beta-1,2-linked mannans. J. Immunol. 177, 4718-4726.

Konstantinov,K.N., Shames,B., Izuno,G., and Liu,F.T. (1994). Expression of epsilon BP, a beta-galactoside-binding soluble lectin, in normal and neoplastic epidermis. Exp. Dermatol. 3, 9-16.

Kuwabara,I. and Liu,F.T. (1996). Galectin-3 promotes adhesion of human neutrophils to laminin. J. Immunol. 156, 3939-3944.

Leask,A. and Abraham,D.J. (2004). TGF-beta signaling and the fibrotic response. FASEB J. 18, 816-827.

Liew,F.Y., Li,Y., Moss,D., Parkinson,C., Rogers,M.V., and Moncada,S. (1991). Resistance to Leishmania major infection correlates with the induction of nitric oxide synthase in murine macrophages. Eur. J. Immunol. 21, 3009-3014.

Lin,H.M., Moon,B.K., Yu,F., and Kim,H.R. (2000). Galectin-3 mediates genisteininduced $\mathrm{G}(2) / \mathrm{M}$ arrest and inhibits apoptosis. Carcinogenesis 21, 1941-1945.

Lin,H.M., Pestell,R.G., Raz,A., and Kim,H.R. (2002). Galectin-3 enhances cyclin $\mathrm{D}(1)$ promoter activity through SP1 and a cAMP-responsive element in human breast epithelial cells. Oncogene 21,8001-8010.

Liu,F.T. (1993). S-type mammalian lectins in allergic inflammation. Immunol. Today 14, 486-490.

Liu,F.T., Hsu,D.K., Zuberi,R.I., Kuwabara,I., Chi,E.Y., and Henderson,W.R., Jr. (1995). Expression and function of galectin-3, a beta-galactoside-binding lectin, in human monocytes and macrophages. Am. J. Pathol. 147, 1016-1028.

Liu,F.T., Patterson,R.J., and Wang,J.L. (2002). Intracellular functions of galectins. Biochim. Biophys. Acta 1572, 263-273.

Liu,H., Drew,P., Gaugler,A.C., Cheng,Y., and Visner,G.A. (2005). Pirfenidone inhibits lung allograft fibrosis through L-arginine-arginase pathway. Am. J. Transplant. 5, 1256-1263.

Liu,L., Sakai,T., Sano,N., and Fukui,K. (2004). Nucling mediates apoptosis by inhibiting expression of galectin-3 through interference with nuclear factor kappaB signalling. Biochem. J. 380, 31-41.

Lobsanov,Y.D., Gitt,M.A., Leffler,H., Barondes,S.H., and Rini,J.M. (1993). X-ray crystal structure of the human dimeric S-Lac lectin, L-14-II, in complex with lactose at 2.9-A resolution. J. Biol. Chem. 268, 27034-27038.

Loke,P., MacDonald,A.S., Robb,A., Maizels,R.M., and Allen,J.E. (2000). Alternatively activated macrophages induced by nematode infection inhibit proliferation via cell-to-cell contact. Eur. J. Immunol. 30, 2669-2678. 
Looney,M.R., Su,X., Van Ziffle,J.A., Lowell,C.A., and Matthay,M.A. (2006). Neutrophils and their Fc gamma receptors are essential in a mouse model of transfusion-related acute lung injury. J. Clin. Invest 116, 1615-1623.

Lotz,M.M., Andrews,C.W., Jr., Korzelius,C.A., Lee,E.C., Steele,G.D., Jr., Clarke,A., and Mercurio,A.M. (1993). Decreased expression of Mac-2 (carbohydrate binding protein 35) and loss of its nuclear localization are associated with the neoplastic progression of colon carcinoma. Proc. Natl. Acad. Sci. U. S. A 90, 3466-3470.

Louis,J., Himmelrich,H., Parra-Lopez,C., Tacchini-Cottier,F., and Launois,P. (1998). Regulation of protective immunity against Leishmania major in mice. Curr. Opin. Immunol. 10, 459-464.

Lowell,C.A., Fumagalli,L., and Berton,G. (1996). Deficiency of Src family kinases p59/61hck and p58c-fgr results in defective adhesion-dependent neutrophil functions. J. Cell Biol. 133, 895-910.

Maeda,N., Kawada,N., Seki,S., Arakawa,T., Ikeda,K., Iwao,H., Okuyama,H., Hirabayashi,J., Kasai,K., and Yoshizato,K. (2003). Stimulation of Proliferation of Rat Hepatic Stellate Cells by Galectin-1 and Galectin-3 through Different Intracellular Signaling Pathways. J. Biol. Chem. 278, 18938-18944.

Mandrell,R.E., Apicella,M.A., Lindstedt,R., and Leffler,H. (1994). Possible interaction between animal lectins and bacterial carbohydrates. Methods Enzymol. $236,231-254$.

Mantovani,A., Sozzani,S., Locati,M., Allavena,P., and Sica,A. (2002). Macrophage polarization: tumor-associated macrophages as a paradigm for polarized M2 mononuclear phagocytes. Trends Immunol. 23, 549-555.

Marra,F., Arrighi,M.C., Fazi,M., Caligiuri,A., Pinzani,M., Romanelli,R.G., Efsen,E., Laffi,G., and Gentilini,P. (1999). Extracellular signal-regulated kinase activation differentially regulates platelet-derived growth factor's actions in hepatic stellate cells, and is induced by in vivo liver injury in the rat. Hepatology 30, 951-958.

Marra,F., Pinzani,M., DeFranco,R., Laffi,G., and Gentilini,P. (1995). Involvement of phosphatidylinositol 3-kinase in the activation of extracellular signal-regulated kinase by PDGF in hepatic stellate cells. FEBS Lett. 376, 141-145.

Massa,S.M., Cooper,D.N., Leffler,H., and Barondes,S.H. (1993). L-29, an endogenous lectin, binds to glycoconjugate ligands with positive cooperativity. Biochemistry 32, 260-267.

Masszi,A., Fan,L., Rosivall,L., McCulloch,C.A., Rotstein,O.D., Mucsi,I., and Kapus,A. (2004). Integrity of cell-cell contacts is a critical regulator of TGF-beta 1induced epithelial-to-myofibroblast transition: role for beta-catenin. Am. J. Pathol. 165, 1955-1967. 
Matarrese,P., Fusco,O., Tinari,N., Natoli,C., Liu,F.T., Semeraro,M.L., Malorni,W., and Iacobelli,S. (2000). Galectin-3 overexpression protects from apoptosis by improving cell adhesion properties. Int. J. Cancer 85, 545-554.

Menon,R.P. and Hughes,R.C. (1999). Determinants in the N-terminal domains of galectin-3 for secretion by a novel pathway circumventing the endoplasmic reticulum-Golgi complex. Eur. J. Biochem. 264, 569-576.

Menon,R.P., Strom,M., and Hughes,R.C. (2000). Interaction of a novel cysteine and histidine-rich cytoplasmic protein with galectin-3 in a carbohydrate-independent manner. FEBS Lett. 470, 227-231.

Mey,A., Leffler,H., Hmama,Z., Normier,G., and Revillard,J.P. (1996). The animal lectin galectin-3 interacts with bacterial lipopolysaccharides via two independent sites. J. Immunol. 156, 1572-1577.

Misson,P., van den,B.S., Barbarin,V., Lison,D., and Huaux,F. (2004). Markers of macrophage differentiation in experimental silicosis. J. Leukoc. Biol. 76, 926-932.

Mizgerd,J.P., Horwitz,B.H., Quillen,H.C., Scott,M.L., and Doerschuk,C.M. (1999). Effects of CD18 deficiency on the emigration of murine neutrophils during pneumonia. J. Immunol. 163, 995-999.

Mizgerd,J.P., Kubo,H., Kutkoski,G.J., Bhagwan,S.D., Scharffetter-Kochanek,K., Beaudet,A.L., and Doerschuk,C.M. (1997). Neutrophil emigration in the skin, lungs, and peritoneum: different requirements for CD11/CD18 revealed by CD18-deficient mice. J. Exp. Med. 186, 1357-1364.

Mizgerd,J.P., Meek,B.B., Kutkoski,G.J., Bullard,D.C., Beaudet,A.L., and Doerschuk,C.M. (1996). Selectins and neutrophil traffic: margination and Streptococcus pneumoniae-induced emigration in murine lungs. J. Exp. Med. 184, 639-645.

Moore,K.W., de Waal,M.R., Coffman,R.L., and O'Garra,A. (2001). Interleukin-10 and the interleukin-10 receptor. Annu. Rev. Immunol. 19, 683-765.

Mora,A.L., Torres-Gonzalez,E., Rojas,M., Corredor,C., Ritzenthaler,J., Xu,J., Roman,J., Brigham,K., and Stecenko,A. (2006). Activation of alveolar macrophages via the alternative pathway in herpesvirus-induced lung fibrosis. Am. J. Respir. Cell Mol. Biol. 35, 466-473.

Moustakas,A. and Heldin,C.H. (2005). Non-Smad TGF-beta signals. J. Cell Sci. 118, 3573-3584.

Moutsatsos,I.K., Wade,M., Schindler,M., and Wang,J.L. (1987). Endogenous lectins from cultured cells: nuclear localization of carbohydrate-binding protein 35 in proliferating 3 T3 fibroblasts. Proc. Natl. Acad. Sci. U. S. A 84, 6452-6456.

Munder,M., Eichmann,K., and Modolell,M. (1998). Alternative metabolic states in murine macrophages reflected by the nitric oxide synthase/arginase balance: 
competitive regulation by CD4+ T cells correlates with Th1/Th2 phenotype. J. Immunol. 160, 5347-5354.

Munder,M., Eichmann,K., Moran,J.M., Centeno,F., Soler,G., and Modolell,M. (1999). Th1/Th2-regulated expression of arginase isoforms in murine macrophages and dendritic cells. J. Immunol. 163, 3771-3777.

Nagaoka,T., Kaburagi,Y., Hamaguchi,Y., Hasegawa,M., Takehara,K., Steeber,D.A., Tedder,T.F., and Sato,S. (2000). Delayed wound healing in the absence of intercellular adhesion molecule-1 or L-selectin expression. Am. J. Pathol. 157, 237247.

Nair,M.G., Cochrane,D.W., and Allen,J.E. (2003). Macrophages in chronic type 2 inflammation have a novel phenotype characterized by the abundant expression of Ym1 and Fizz1 that can be partly replicated in vitro. Immunol. Lett. 85, 173-180.

Nair,M.G., Gallagher,I.J., Taylor,M.D., Loke,P., Coulson,P.S., Wilson,R.A., Maizels,R.M., and Allen,J.E. (2005). Chitinase and Fizz family members are a generalized feature of nematode infection with selective upregulation of Ym1 and Fizzl by antigen-presenting cells. Infect. Immun. 73, 385-394.

Nakao,A., Afrakhte,M., Moren,A., Nakayama,T., Christian,J.L., Heuchel,R., Itoh,S., Kawabata,M., Heldin,N.E., Heldin,C.H., and ten Dijke,P. (1997). Identification of Smad7, a TGFbeta-inducible antagonist of TGF-beta signalling. Nature 389, 631635.

Nangia-Makker,P., Honjo,Y., Sarvis,R., Akahani,S., Hogan,V., Pienta,K.J., and Raz,A. (2000). Galectin-3 induces endothelial cell morphogenesis and angiogenesis. Am. J. Pathol. 156, 899-909.

Neuberger,J. (2000). Liver transplantation. J. Hepatol. 32, 198-207.

Nieminen,J., Kuno,A., Hirabayashi,J., and Sato,S. (2006). Visualization of galectin-3 oligomerization on the surface of neutrophils and endothelial cells using fluorescence resonance energy transfer. J. Biol. Chem.

Nieminen,J., Kuno,A., Hirabayashi,J., and Sato,S. (2007). Visualization of galectin-3 oligomerization on the surface of neutrophils and endothelial cells using fluorescence resonance energy transfer. J. Biol. Chem. 282, 1374-1383.

Nieminen,J., St Pierre,C., and Sato,S. (2005). Galectin-3 interacts with naive and primed neutrophils, inducing innate immune responses. J. Leukoc. Biol. 78, 11271135 .

Noel,W., Raes,G., Hassanzadeh,G.G., De Baetselier,P., and Beschin,A. (2004). Alternatively activated macrophages during parasite infections. Trends Parasitol. 20, 126-133. 
Novak,R., Henriques,B., Charpentier,E., Normark,S., and Tuomanen,E. (1999). Emergence of vancomycin tolerance in Streptococcus pneumoniae. Nature 399, 590 593.

O'Neill,S.M., Brady,M.T., Callanan,J.J., Mulcahy,G., Joyce,P., Mills,K.H., and Dalton,J.P. (2000). Fasciola hepatica infection downregulates Th1 responses in mice. Parasite Immunol. 22, 147-155.

Obaro,S. and Adegbola,R. (2002). The pneumococcus: carriage, disease and conjugate vaccines. J. Med. Microbiol. 51, 98-104.

Ochieng,J., Furtak,V., and Lukyanov,P. (2004). Extracellular functions of galectin-3. Glycoconj. J. 19, 527-535.

Ochieng,J., Leite-Browning,M.L., and Warfield,P. (1998). Regulation of cellular adhesion to extracellular matrix proteins by galectin-3. Biochem. Biophys. Res. Commun. 246, 788-791.

Ochieng,J. and Warfield,P. (1995). Galectin-3 binding potentials of mouse tumor EHS and human placental laminins. Biochem. Biophys. Res. Commun. 217, 402406.

Ochieng,J., Warfield,P., Green-Jarvis,B., and Fentie,I. (1999). Galectin-3 regulates the adhesive interaction between breast carcinoma cells and elastin. J. Cell Biochem. $75,505-514$.

Oka,N., Nakahara,S., Takenaka,Y., Fukumori,T., Hogan,V., Kanayama,H.O., Yanagawa,T., and Raz,A. (2005). Galectin-3 inhibits tumor necrosis factor-related apoptosis-inducing ligand-induced apoptosis by activating Akt in human bladder carcinoma cells. Cancer Res. 65, 7546-7553.

Okamoto,H., Mizuno,K., and Horio,T. (2003). Monocyte-derived multinucleated giant cells and sarcoidosis. J. Dermatol. Sci. 31, 119-128.

Pacis,R.A., Pilat,M.J., Pienta,K.J., Wojno,K., Raz,A., Hogan,V., and Cooper,C.R. (2000). Decreased galectin-3 expression in prostate cancer. Prostate 44, 118-123.

Park,J.W., Voss,P.G., Grabski,S., Wang,J.L., and Patterson,R.J. (2001). Association of galectin-1 and galectin-3 with Gemin4 in complexes containing the SMN protein. Nucleic Acids Res. 29, 3595-3602.

Parmacek,M.S., Karpinski,B.A., Gottesdiener,K.M., Thompson,C.B., and Leiden,J.M. (1989). Structure, expression and regulation of the murine 4F2 heavy chain. Nucleic Acids Res. 17, 1915-1931.

Paron,I., Scaloni,A., Pines,A., Bachi,A., Liu,F.T., Puppin,C., Pandolfi,M., Ledda,L., Di Loreto,C., Damante,G., and Tell,G. (2003). Nuclear localization of Galectin-3 in transformed thyroid cells: a role in transcriptional regulation. Biochem. Biophys. Res. Commun. 302, 545-553. 
Partridge,E.A., Le Roy,C., Di Guglielmo,G.M., Pawling,J., Cheung,P., Granovsky,M., Nabi,I.R., Wrana,J.L., and Dennis,J.W. (2004). Regulation of cytokine receptors by Golgi N-glycan processing and endocytosis. Science 306, 120 124.

Paterson,G.K., Blue,C.E., and Mitchell,T.J. (2005). Role of interleukin-18 in experimental infections with Streptococcus pneumoniae. J. Med. Microbiol. 54, $323-$ 326.

Petray,P., Castanos-Velez,E., Grinstein,S., Orn,A., and Rottenberg,M.E. (1995). Role of nitric oxide in resistance and histopathology during experimental infection with Trypanosoma cruzi. Immunol. Lett. 47, 121-126.

Pieters,R.J. (2006). Inhibition and Detection of Galectins. Chembiochem.

Poynard,T., McHutchison,J., Manns,M., Trepo,C., Lindsay,K., Goodman,Z., Ling,M.H., and Albrecht,J. (2002). Impact of pegylated interferon alfa-2b and ribavirin on liver fibrosis in patients with chronic hepatitis C. Gastroenterology 122, 1303-1313.

Puglisi,F., Minisini,A.M., Barbone,F., Intersimone,D., Aprile,G., Puppin,C., Damante,G., Paron,I., Tell,G., Piga,A., and Di Loreto,C. (2004). Galectin-3 expression in non-small cell lung carcinoma. Cancer Lett. 212, 233-239.

Qi,Z., Atsuchi,N., Ooshima,A., Takeshita,A., and Ueno,H. (1999). Blockade of type beta transforming growth factor signaling prevents liver fibrosis and dysfunction in the rat. Proc. Natl. Acad. Sci. U. S. A 96, 2345-2349.

Quackenbush,E., Clabby,M., Gottesdiener,K.M., Barbosa,J., Jones,N.H., Strominger,J.L., Speck,S., and Leiden,J.M. (1987). Molecular cloning of complementary DNAs encoding the heavy chain of the human 4F2 cell-surface antigen: a type II membrane glycoprotein involved in normal and neoplastic cell growth. Proc. Natl. Acad. Sci. U. S. A 84, 6526-6530.

Quan,T.E., Cowper,S.E., and Bucala,R. (2006). The role of circulating fibrocytes in fibrosis. Curr. Rheumatol. Rep. 8, 145-150.

Raes,G., Brys,L., Dahal,B.K., Brandt,J., Grooten,J., Brombacher,F., Vanham,G., Noel,W., Bogaert,P., Boonefaes,T., Kindt,A., Van den,B.R., Leenen,P.J., De Baetselier,P., and Ghassabeh,G.H. (2005). Macrophage galactose-type C-type lectins as novel markers for alternatively activated macrophages elicited by parasitic infections and allergic airway inflammation. J. Leukoc. Biol. 77, 321-327.

Raes,G., Noel,W., Beschin,A., Brys,L., De Baetselier,P., and Hassanzadeh,G.H. (2002). FIZZ1 and Ym as tools to discriminate between differentially activated macrophages. Dev. Immunol. 9, 151-159.

Raimond,J., Zimonjic,D.B., Mignon,C., Mattei,M., Popescu,N.C., Monsigny,M., and Legrand,A. (1997). Mapping of the galectin-3 gene (LGALS3) to human chromosome 14 at region 14q21-22. Mamm. Genome 8, 706-707. 
Rauh,M.J., Ho,V., Pereira,C., Sham,A., Sly,L.M., Lam,V., Huxham,L., Minchinton,A.I., Mui,A., and Krystal,G. (2005). SHIP represses the generation of alternatively activated macrophages. Immunity. 23, 361-374.

Raz,A., Avivi,A., Pazerini,G., and Carmi,P. (1987). Cloning and expression of cDNA for two endogenous UV-2237 fibrosarcoma lectin genes. Exp. Cell Res. 173, 109-116.

Reiman,R.M., Thompson,R.W., Feng,C.G., Hari,D., Knight,R., Cheever,A.W., Rosenberg,H.F., and Wynn,T.A. (2006). Interleukin-5 (IL-5) augments the progression of liver fibrosis by regulating IL-13 activity. Infect. Immun. 74, 14711479.

Rintoul,R.C., Buttery,R.C., Mackinnon,A.C., Wong,W.S., Mosher,D., Haslett,C., and Sethi,T. (2002). Cross-linking CD98 promotes integrin-like signaling and anchorage-independent growth. Mol. Biol. Cell 13, 2841-2852.

Roberts,A.B. (1999). TGF-beta signaling from receptors to the nucleus. Microbes. Infect. 1, 1265-1273.

Rodriguez-Vita,J., Sanchez-Lopez,E., Esteban,V., Ruperez,M., Egido,J., and RuizOrtega,M. (2005). Angiotensin II activates the Smad pathway in vascular smooth muscle cells by a transforming growth factor-beta-independent mechanism. Circulation 111, 2509-2517.

Saada,A., Reichert,F., and Rotshenker,S. (1996). Granulocyte macrophage colony stimulating factor produced in lesioned peripheral nerves induces the up-regulation of cell surface expression of MAC-2 by macrophages and Schwann cells. J. Cell Biol. 133, 159-167.

Sano,H., Hsu,D.K., Apgar,J.R., Yu,L., Sharma,B.B., Kuwabara,I., Izui,S., and Liu,F.T. (2003). Critical role of galectin-3 in phagocytosis by macrophages. J. Clin. Invest 112, 389-397.

Sano,H., Hsu,D.K., Yu,L., Apgar,J.R., Kuwabara,I., Yamanaka,T., Hirashima,M., and Liu,F.T. (2000). Human galectin-3 is a novel chemoattractant for monocytes and macrophages. J. Immunol. 165, 2156-2164.

Sato,S. and Hughes,R.C. (1992). Binding specificity of a baby hamster kidney lectin for H type I and II chains, polylactosamine glycans, and appropriately glycosylated forms of laminin and fibronectin. J. Biol. Chem. 267, 6983-6990.

Sato,S. and Hughes,R.C. (1994). Regulation of secretion and surface expression of Mac-2, a galactoside-binding protein of macrophages. J. Biol. Chem. 269, 44244430 .

Sato,S. and Nieminen,J. (2004). Seeing strangers or announcing "danger": galectin-3 in two models of innate immunity. Glycoconj. J. 19, 583-591. 
Sato,S., Ouellet,N., Pelletier,I., Simard,M., Rancourt,A., and Bergeron,M.G. (2002). Role of galectin-3 as an adhesion molecule for neutrophil extravasation during streptococcal pneumonia. J. Immunol. 168, 1813-1822.

Savill,J., Dransfield,I., Gregory,C., and Haslett,C. (2002). A blast from the past: clearance of apoptotic cells regulates immune responses. Nat. Rev. Immunol. 2, 965975.

Savill,J.S., Wyllie,A.H., Henson,J.E., Walport,M.J., Henson,P.M., and Haslett,C. (1989). Macrophage phagocytosis of aging neutrophils in inflammation.

Programmed cell death in the neutrophil leads to its recognition by macrophages. J. Clin. Invest $83,865-875$.

Schabbauer,G., Tencati,M., Pedersen,B., Pawlinski,R., and Mackman,N. (2004). PI3K-Akt pathway suppresses coagulation and inflammation in endotoxemic mice. Arterioscler. Thromb. Vasc. Biol. 24, 1963-1969.

Schaffert,C., Pour,P.M., and Chaney,W.G. (1998). Localization of galectin-3 in normal and diseased pancreatic tissue. Int. J. Pancreatol. 23, 1-9.

Schoeppner,H.L., Raz,A., Ho,S.B., and Bresalier,R.S. (1995). Expression of an endogenous galactose-binding lectin correlates with neoplastic progression in the colon. Cancer 75, 2818-2826.

Seve,A.P., Felin,M., Doyennette-Moyne,M.A., Sahraoui,T., Aubery,M., and Hubert,J. (1993). Evidence for a lactose-mediated association between two nuclear carbohydrate-binding proteins. Glycobiology 3, 23-30.

Shimura,T., Takenaka,Y., Fukumori,T., Tsutsumi,S., Okada,K., Hogan,V., Kikuchi,A., Kuwano,H., and Raz,A. (2005). Implication of galectin-3 in Wnt signaling. Cancer Res. 65, 3535-3537.

Shimura,T., Takenaka,Y., Tsutsumi,S., Hogan,V., Kikuchi,A., and Raz,A. (2004). Galectin-3, a novel binding partner of beta-catenin. Cancer Res. 64, 6363-6367.

Sibille,Y. and Reynolds,H.Y. (1990). Macrophages and polymorphonuclear neutrophils in lung defense and injury. Am. Rev. Respir. Dis. 141, 471-501.

Sica,A., Schioppa,T., Mantovani,A., and Allavena,P. (2006). Tumour-associated macrophages are a distinct M2 polarised population promoting tumour progression: potential targets of anti-cancer therapy. Eur. J. Cancer 42, 717-727.

Song,E., Ouyang,N., Horbelt,M., Antus,B., Wang,M., and Exton,M.S. (2000). Influence of alternatively and classically activated macrophages on fibrogenic activities of human fibroblasts. Cell Immunol. 204, 19-28.

Soutschek,J., Akinc,A., Bramlage,B., Charisse,K., Constien,R., Donoghue,M., Elbashir,S., Geick,A., Hadwiger,P., Harborth,J., John,M., Kesavan,V., Lavine,G., Pandey,R.K., Racie,T., Rajeev,K.G., Rohl,I., Toudjarska,I., Wang,G., Wuschko,S., Bumcrot,D., Koteliansky,V., Limmer,S., Manoharan,M., and Vornlocher,H.P. 
(2004). Therapeutic silencing of an endogenous gene by systemic administration of modified siRNAs. Nature 432, 173-178.

Stein,M., Keshav,S., Harris,N., and Gordon,S. (1992). Interleukin 4 potently enhances murine macrophage mannose receptor activity: a marker of alternative immunologic macrophage activation. J. Exp. Med. 176, 287-292.

Stout,R.D., Jiang,C., Matta,B., Tietzel,I., Watkins,S.K., and Suttles,J. (2005). Macrophages sequentially change their functional phenotype in response to changes in microenvironmental influences. J. Immunol. 175, 342-349.

Strieter,R.M. and Keane,M.P. (2004). Innate immunity dictates cytokine polarization relevant to the development of pulmonary fibrosis. J. Clin. Invest 114, 165-168.

Surendran,K., McCaul,S.P., and Simon,T.C. (2002). A role for Wnt-4 in renal fibrosis. Am. J. Physiol Renal Physiol 282, F431-F441.

Swarte,V.V., Mebius,R.E., Joziasse,D.H., Van den Eijnden,D.H., and Kraal,G. (1998). Lymphocyte triggering via L-selectin leads to enhanced galectin-3-mediated binding to dendritic cells. Eur. J. Immunol. 28, 2864-2871.

Tajima,M., Higuchi,S., Higuchi,Y., Miyamoto,N., Uchida,A., Ito,M., Nishio,M., Komada,H., Kawano,M., Kusagawa,S., Tsurudome,M., and Ito,Y. (1999).

Suppression of FRP-1/CD98-mediated multinucleated giant cell and osteoclast formation by an anti-FRP-1/CD98 mAb, HBJ 127, that inhibits c-src expression. Cell Immunol. 193, 162-169.

Takenaka,Y., Fukumori,T., and Raz,A. (2004). Galectin-3 and metastasis. Glycoconj. J. 19, 543-549.

Tanaka,S., Deai,K., Konomi,A., Takahashi,K., Yamane,H., Sugimoto,Y., and Ichikawa,A. (2004). Expression of L-histidine decarboxylase in granules of elicited mouse polymorphonuclear leukocytes. Eur. J. Immunol. 34, 1472-1482.

Teder,P., Vandivier,R.W., Jiang,D., Liang,J., Cohn,L., Pure,E., Henson,P.M., and Noble,P.W. (2002). Resolution of lung inflammation by CD44. Science 296, 155158.

Teichberg,V.I., Silman,I., Beitsch,D.D., and Resheff,G. (1975). A beta-D-galactoside binding protein from electric organ tissue of Electrophorus electricus. Proc. Natl. Acad. Sci. U. S. A 72, 1383-1387.

Thalheimer,U. and Capra,F. (2002). Liver transplantation: making the best out of what we have. Dig. Dis. Sci. 47, 945-953.

Traore,K., Trush,M.A., George,M., Jr., Spannhake,E.W., Anderson,W., and Asseffa,A. (2005). Signal transduction of phorbol 12-myristate 13-acetate (PMA)induced growth inhibition of human monocytic leukemia THP-1 cells is reactive oxygen dependent. Leuk. Res. 29, 863-879. 
Tsai,W.C., Strieter,R.M., Zisman,D.A., Wilkowski,J.M., Bucknell,K.A., Chen,G.H., and Standiford,T.J. (1997). Nitric oxide is required for effective innate immunity against Klebsiella pneumoniae. Infect. Immun. 65, 1870-1875.

Tsuchiya,S., Kobayashi,Y., Goto,Y., Okumura,H., Nakae,S., Konno,T., and Tada,K. (1982). Induction of maturation in cultured human monocytic leukemia cells by a phorbol diester. Cancer Res. 42, 1530-1536.

Tsukada,S., Westwick,J.K., Ikejima,K., Sato,N., and Rippe,R.A. (2005). SMAD and p38 MAPK signaling pathways independently regulate alpha1(I) collagen gene expression in unstimulated and transforming growth factor-beta-stimulated hepatic stellate cells. J. Biol. Chem. 280, 10055-10064.

Uemura,M., Swenson,E.S., Gaca,M.D., Giordano,F.J., Reiss,M., and Wells,R.G. (2005). Smad2 and Smad3 play different roles in rat hepatic stellate cell function and alpha-smooth muscle actin organization. Mol. Biol. Cell 16, 4214-4224.

Van Den Brule,F.A., Waltregny,D., Liu,F.T., and Castronovo,V. (2000). Alteration of the cytoplasmic/nuclear expression pattern of galectin-3 correlates with prostate carcinoma progression. Int. J. Cancer 89, 361-367.

van den,B.F., Califice,S., and Castronovo,V. (2004). Expression of galectins in cancer: a critical review. Glycoconj. J. 19, 537-542.

Verrey,F., Closs,E.I., Wagner,C.A., Palacin,M., Endou,H., and Kanai,Y. (2004). CATs and HATs: the SLC7 family of amino acid transporters. Pflugers Arch. 447, 532-542.

Volante,M., Bozzalla-Cassione,F., Orlandi,F., and Papotti,M. (2004). Diagnostic role of galectin-3 in follicular thyroid tumors. Virchows Arch. 444, 309-312.

Walrand,S., Valeix,S., Rodriguez,C., Ligot,P., Chassagne,J., and Vasson,M.P. (2003). Flow cytometry study of polymorphonuclear neutrophil oxidative burst: a comparison of three fluorescent probes. Clin. Chim. Acta 331, 103-110.

Wang,J.F., Jiao,H., Stewart,T.L., Shankowsky,H.A., Scott,P.G., and Tredget,E.E. (2007). Fibrocytes from burn patients regulate the activities of fibroblasts. Wound. Repair Regen. 15, 113-121.

Wang,J.L., Gray,R.M., Haudek,K.C., and Patterson,R.J. (2004). Nucleocytoplasmic lectins. Biochim. Biophys. Acta 1673, 75-93.

Wang,J.L., Werner,E.A., Laing,J.G., and Patterson,R.J. (1992). Nuclear and cytoplasmic localization of a lectin-ribonucleoprotein complex. Biochem. Soc. Trans. 20, 269-274.

Wang,L., Friess,H., Zhu,Z., Frigeri,L., Zimmermann,A., Korc,M., Berberat,P.O., and Buchler,M.W. (2000). Galectin-1 and galectin-3 in chronic pancreatitis. Lab Invest $80,1233-1241$. 
Wang,S., Wilkes,M.C., Leof,E.B., and Hirschberg,R. (2005). Imatinib mesylate blocks a non-Smad TGF-beta pathway and reduces renal fibrogenesis in vivo. FASEB J. 19, 1-11.

Welch,J.S., Escoubet-Lozach,L., Sykes,D.B., Liddiard,K., Greaves,D.R., and Glass,C.K. (2002). TH2 cytokines and allergic challenge induce Ym1 expression in macrophages by a STAT6-dependent mechanism. J. Biol. Chem. 277, 42821-42829.

Wilkes,M.C., Murphy,S.J., Garamszegi,N., and Leof,E.B. (2003). Cell-type-specific activation of PAK2 by transforming growth factor beta independent of Smad2 and Smad3. Mol. Cell Biol. 23, 8878-8889.

Wynn,T.A. (2004). Fibrotic disease and the $\mathrm{T}(\mathrm{H}) 1 / \mathrm{T}(\mathrm{H}) 2$ paradigm. Nat. Rev. Immunol. 4, 583-594.

Wynn,T.A. (2007). Common and unique mechanisms regulate fibrosis in various fibroproliferative diseases. J. Clin. Invest 117, 524-529.

Wynn,T.A., Cheever,A.W., Jankovic,D., Poindexter,R.W., Caspar,P., Lewis,F.A., and Sher,A. (1995). An IL-12-based vaccination method for preventing fibrosis induced by schistosome infection. Nature 376, 594-596.

Xu,X.C., el Naggar,A.K., and Lotan,R. (1995). Differential expression of galectin-1 and galectin-3 in thyroid tumors. Potential diagnostic implications. Am. J. Pathol. $147,815-822$.

Yamaoka,A., Kuwabara,I., Frigeri,L.G., and Liu,F.T. (1995). A human lectin, galectin-3 (epsilon bp/Mac-2), stimulates superoxide production by neutrophils. J. Immunol. 154, 3479-3487.

Yang,R.Y., Hsu,D.K., and Liu,F.T. (1996). Expression of galectin-3 modulates Tcell growth and apoptosis. Proc. Natl. Acad. Sci. U. S. A 93, 6737-6742.

Young,A.N., Amin,M.B., Moreno,C.S., Lim,S.D., Cohen,C., Petros,J.A., Marshall,F.F., and Neish,A.S. (2001). Expression profiling of renal epithelial neoplasms: a method for tumor classification and discovery of diagnostic molecular markers. Am. J. Pathol. 158, 1639-1651.

Yu,F., Finley,R.L., Jr., Raz,A., and Kim,H.R. (2002). Galectin-3 translocates to the perinuclear membranes and inhibits cytochrome c release from the mitochondria. A role for synexin in galectin-3 translocation. J. Biol. Chem. 277, 15819-15827.

Zeisberg,M. and Kalluri,R. (2004). The role of epithelial-to-mesenchymal transition in renal fibrosis. J. Mol. Med. 82, 175-181.

Zuberi,R.I., Hsu,D.K., Kalayci,O., Chen,H.Y., Sheldon,H.K., Yu,L., Apgar,J.R., Kawakami,T., Lilly,C.M., and Liu,F.T. (2004). Critical role for galectin-3 in airway inflammation and bronchial hyperresponsiveness in a murine model of asthma. Am. J. Pathol. 165, 2045-2053. 